\title{
Toxicogenomics responses in the in vitro liver : a view on human interindividual variation
}

Citation for published version (APA):

Jetten, M. J. A. (2014). Toxicogenomics responses in the in vitro liver : a view on human interindividual variation. [Doctoral Thesis, Maastricht University]. Maastricht University.

https://doi.org/10.26481/dis.20141205mj

Document status and date:

Published: 01/01/2014

DOI:

10.26481/dis.20141205mj

Document Version:

Publisher's PDF, also known as Version of record

\section{Please check the document version of this publication:}

- A submitted manuscript is the version of the article upon submission and before peer-review. There can be important differences between the submitted version and the official published version of record.

People interested in the research are advised to contact the author for the final version of the publication, or visit the DOI to the publisher's website.

- The final author version and the galley proof are versions of the publication after peer review.

- The final published version features the final layout of the paper including the volume, issue and page numbers.

Link to publication

\footnotetext{
General rights rights.

- You may freely distribute the URL identifying the publication in the public portal. please follow below link for the End User Agreement:

www.umlib.nl/taverne-license

Take down policy

If you believe that this document breaches copyright please contact us at:

repository@maastrichtuniversity.nl

providing details and we will investigate your claim.
}

Copyright and moral rights for the publications made accessible in the public portal are retained by the authors and/or other copyright owners and it is a condition of accessing publications that users recognise and abide by the legal requirements associated with these

- Users may download and print one copy of any publication from the public portal for the purpose of private study or research.

- You may not further distribute the material or use it for any profit-making activity or commercial gain

If the publication is distributed under the terms of Article $25 \mathrm{fa}$ of the Dutch Copyright Act, indicated by the "Taverne" license above, 


\title{
Toxicogenomics responses
} in the in vitro liver

\author{
A view on human \\ interindividual variation
}


ISBN: 9789462950115

(C) Marlon J.A. Jetten

Coverdesign: Paul van Cruchten

Lay-out: Paul van Cruchten

Production: Proefschriftmaken.nl || Uitgeverij BOXPress

\section{GROШ}

School for Oncology and Developmental Biology

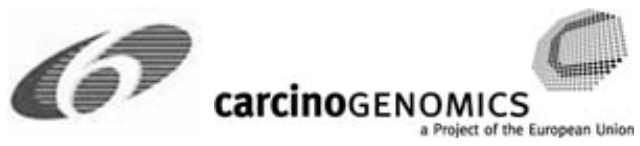

The studies presented in this thesis were performed at the School for Oncology and Developmental Biology (GROW), accredited by the Royal Netherlands Academy of Arts and Sciences (KNAW). The research was funded by the carcinoGENOMICS project [LSHB-CT-2006-037712] an FP6 project from the European Union and the ASAT (Assuring Safety without Animal Testing) program an initiative from the Dutch ministry of Health, Welfare and Sports. 


\section{Toxicogenomics responses in the in vitro liver \\ A view on human interindividual variation}

PROEFSCHRIFT

ter verkrijging van de graad van doctor aan de Universiteit Maastricht, op gezag van de Rector Magnificus, Prof. dr. L. L .G. Soete volgens het besluit van het College van Decanen, in het openbaar te verdedigen op vrijdag 5 december 2014 om 10.00 uur

door

Marlon Jacoba Anne Jetten geboren te Roermond op 24 juni 1983 


\section{Promotor}

Prof. Dr. J.C.S. Kleinjans

\section{Beoordelingscommissie}

Prof. Dr. F.C.S. Ramaekers (voorzitter)

Prof. Dr. C.H.C. Dejong

Prof. Dr. G.M.M. Groothuis (Rijks Universiteit Groningen)

Prof. Dr. H. van Loveren

Prof. Dr. B. van de Water (Universiteit Leiden) 


\section{contents}

Chapter 1

General introduction

Chapter 2

Interindividual variation in response to xenobiotic exposure established in precision-cut human liver slices

Chapter 3

Interindividual variation in gene expression responses and metabolite formation in acetaminophen-exposed primary human hepatocytes

Chapter 4

Baseline and genotoxic compound induced gene expression profiles in HepG2 and HepaRG compared to primary human hepatocytes.

Chapter 5

Does the epigenome predispose for susceptibility to

DNA damage?

Chapter 6

'Omics analysis of low dose acetaminophen intake demonstrates novel response pathways in humans

Chapter 7

Summary and general discussion

Samenvatting en algemene discussie

Addendum I

Valorization

Dankwoord 


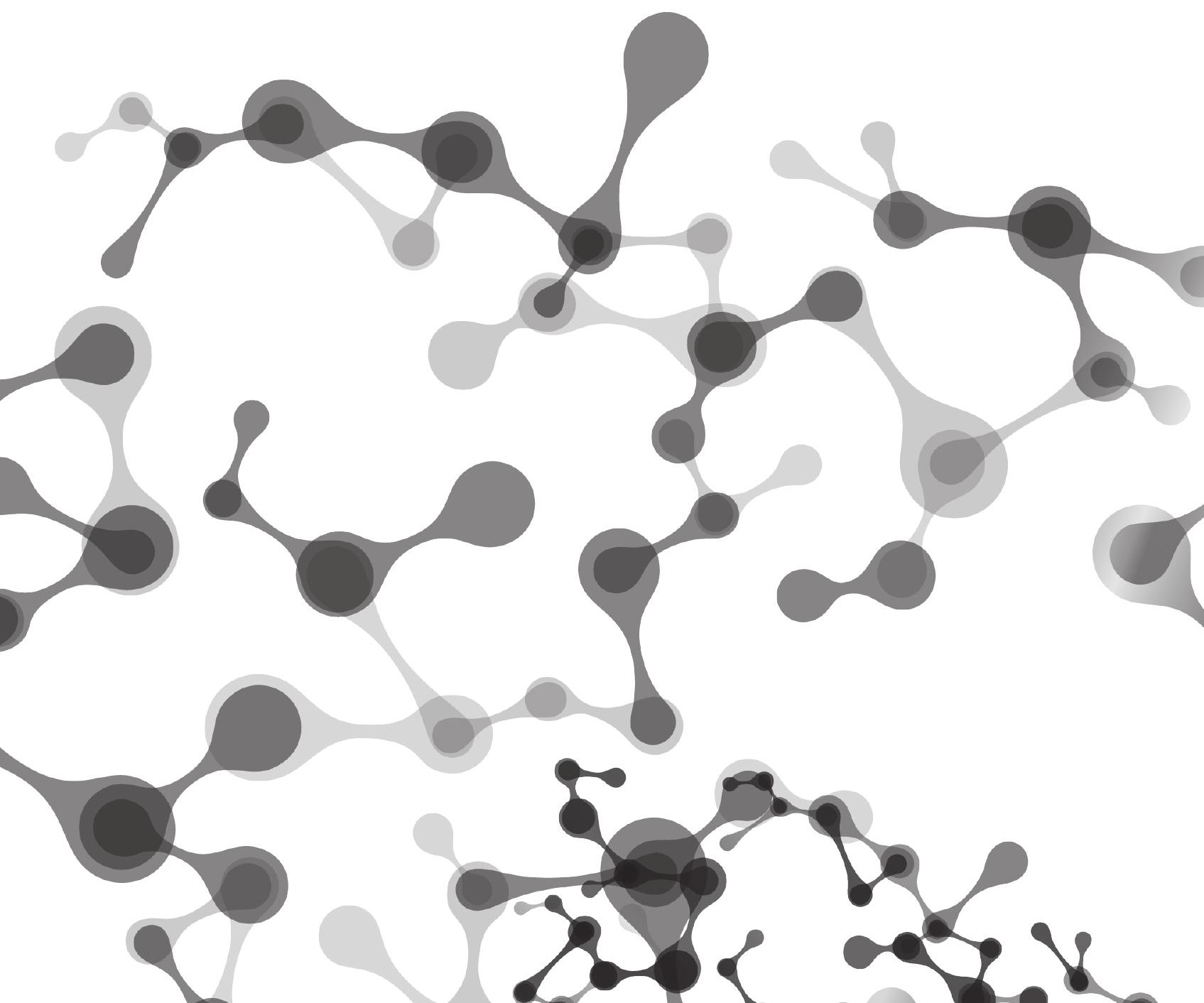




\section{Chapter 1 \\ General introduction}

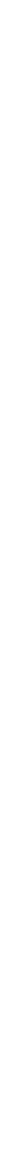




\section{Adverse effects to xenobiotic exposure}

Xenobiotics are defined as any chemical substance of which the occurrence of, or the unusually high concentration in an organism is abnormal. Thus, xenobiotics would typically not be produced by, or be present in an organism. Nevertheless, contact to xenobiotics is an inevitable part of daily life for any organism [1]. For instance, the inhalation of products of combustion whiles breathing or the consumption of food contaminants while feeding are unavoidable scenarios of xenobiotic exposure. Some of the xenobiotics humans are exposed to through air inhalation or food consumption are well known chemical carcinogens; in other words these xenobiotics are chemicals that are known to cause cancer [2].

Chemical carcinogenesis, e.g. the development of cancer after exposure to a chemical, is a multi-step process. In the first step, the initiation phase, a mutation is introduced in the genetic code. In chemical carcinogenesis this is usually caused through covalent binding of a reactive metabolite to the DNA-strand, leading to a DNA-strand break which, if falsely repaired, causes a mutation in the DNA $[3,4]$. When these mutations appear in genes involved in for instance cell differentiation/growth or tumor suppression/promotion, the development of cancerous cells can be induced [5]. The harm done during the initiation phase can still be reversed by the DNA-repair mechanisms. However, when these mechanisms fail mutations become final and a second stage, the promotion stage is entered. This phase promotes cell growth of the mutated cell over a longer period in time leading to the development of non-malignant tumors. Then, during the progression phase, the already mutated cells can acquire even more mutations resulting in unlimited cell growth that will eventually become invasive leading to malignant and metastatic forms of cancer [6].

Another important source of xenobiotics in humans is caused by the exposure to pharmaceuticals/drugs. Organ-related toxicity responses are amongst the unwanted side-effects from xenobiotics coming from the exposure to pharmaceutical chemicals [7]. Adverse reactions to drugs are major issues concerning drug treatment and development, resulting in a high number of deaths and medical costs [8]. Furthermore, idiosyncratic responses impose a financial burden on the pharmacological industry $[7,9]$. 


\section{Interindividual variation in the human population}

Interindividual variation in adverse responses to xenobiotic exposure within the human population is a well-known phenomenon $[10,11]$. For instance, though smoking causes lung cancer, not all individuals that smoke will eventually succumb to this disease. Also, individuals exhibit different side-effects when exposed to similar doses of a pharmaceutical. Environmental factors, but also genetic factors have been proposed as causative elements for the interindividual variation within the human population $[10,11]$. Many studies have focused on finding a single or maybe a few factors explaining the variation within a population in response to xenobiotics, for instance the expression level of a single or a few genes [12]. However, a single factor in general does not seem to be able to provide sufficient

explanation for the interindividual differences in a population, implying that the variation is rather caused by multiple factors [12]. Nevertheless, up till now the (genetic) factors causing interindividual variation are still largely unknown.

\section{Animal models and human risk assessment}

In an attempt to elucidate the factors influencing sensitivity of individuals towards xenobiotic exposure and in order to establish a system for risk assessment of human xenobiotic exposure, researchers have resorted to the use of in vivo animal models; especially rodent models (mice, rats, etc.). In addition, for the preservation of the current levels of human health and environmental protection many regulatory bodies emphasize the need for toxicity testing [13]. As a result, the use of animal models has become deeply manifested into the rules and regulations around toxicity testing, as such becoming an obligatory hurdle to take to ensure human safety [14]. However, the use of animal models brings along a heavy financial burden, and also goes hand-inhand with ethical and moral dilemmas [15]. In addition, over the recent years the reliability and applicably of animal model-derived data towards the human situation has been questioned [15].

Currently, risk-assessment methods based on data-extrapolation from the animal to the human situation make use of a golden standard, the 100-fold safety factor [16]. This factor is applied to the outcome of animal model-derived data in order to establish a dose for a compound/xenobiotic that should be safe for human use. The factor is composed of two parts; a 10-fold margin of safety allowing for the extrapolation from the animal to human situation, and another 10- 
fold margin of safety is included to account for human interindividual variation in susceptibility $[16,17]$. Especially the latter 10-fold factor for interindividual variation is a topic of discussion at this moment in time [18]. An overestimation of interindividual variation through this factor, i.e. the actual interindividual variation is lower than 10-fold, could for instance lead to unnecessarily strict restrictions on the allowable dose-range of a pharmaceutical intended for human use. On the other hand, in case the 10-fold margin is an underestimation of the actual interindividual variation, sensitive individuals within the population could be exposed to a dose of a compound/xenobiotic that imposes a threat to their health/safety. Inbred rodent models by default are not able to reflect the interindividual variation within the human population, which apparently is a crucial aspect in human risk assessment. Therefore the scientific-community has been driven to develop other, better, in vitro models preferably optimised for application to the human situation.

\section{Alternatives to animal models for human risk assessment}

The liver has unique biochemical properties and is the major organ involved in the metabolism of many xenobiotics [19]. As a consequence it is also the main organ that is affected by harmful doses of xenobiotics, resulting in liver toxicity. Due to these aspects, the human liver is the prime candidate for a model for human toxicity in response to xenobiotic exposure. The hepatocyte is the main cell type in the liver; about $60 \%$ of all cells in the liver are hepatocytes. This cell type performs most of the livers functions and, due to their relatively large size take up about $80 \%$ of the livers volume. The other $40 \%$ of the cell-population in the liver are in general smaller cells and account for only about $6.5 \%$ of the livers volume. These cells include; several endothelial cell types lining blood vessels and bile ducts, Kupffer cells, hepatic stellate cells, immune-related cells, fibroblasts and stem cells [20-22]. Several human liver-related models have been developed over the years, which will be discussed in further detail below.

- $\quad$ Carcinoma-based, immortalized cell lines

Several in vitro models based on these cells have been devel oped, two of the most popular ones being HepG2 and HepaRG cells. While the HepG2 cell line has been around since the early 1980 's $[23,24]$, the HepaRG cell line was added more recently to the scale of human liver-based in vitro models; about 20 years later on in the early 2000's [25-27]. Some major advantages of immortalized cells are their long lifespan and their ability to divide, making it possible to culture as many cells as desired. 
These features attribute to an easy accessibility of these cells and relatively low financial costs associated to the use of these in vitro models. This has led to a world-wide application of these cell models, resulting in a high availability of data derived from these in vitro models, especially for HepG2. Data available on the exposure of these cells to xenobiotics suggest that they meet the requirements to study the toxicity-related effects of these compounds [28-32]. However, there are also some disadvantages to the use of these in vitro cell models. One of them being that, in comparison to the liver, these cells exhibit lower levels of many of the xenobiotic metabolizing enzymes (Phase I and Phase II biotransformation enzymes) and of the cellular transporters [31-36]. It has to be noted though that out of the cell models mentioned above, HepaRG cells in general are believed to have levels of Phase I enzyme activities that are more comparable to human liver $[25,27,37-40]$. The aberrant expression of the xenobiotic metabolizing enzymes can at least partially be blamed to the tumorigenic background of these in vitro models. Also, although the carcinoma-related background from these cells makes it possible to culture them over-and-over again, it also discriminates them from the hepatocytes that populate the normal liver. Another, major disadvantage of these in vitro models is that, due to their carcinoma-derived background and thus their single-donor genetic background, none of them is able to reflect human interindividual variation. Therefore, other in vitro models need to be taken into consideration.

Primary human hepatocytes

Primary human hepatocytes are hepatic cells derived directly from the human liver, which are largely able to retain their liver-related functionality $[35,41]$. They can be put into culture immediately after isolation from the liver, or they can be frozen and kept in liquid nitrogen to be put into culture on a later point in time. For optimal performance of PHH in culture, they should be cultured using a sandwich configuration. In a sandwich culture, the cells are provided with a gel-like structural matrix both underneath and on top of the cell layer. By doing so, the cells are provided with a 3D-surrounding mimicking the actual in vivo situation in the liver. As a consequence, these cells seem to preserve most of their liver specific functions including the genetic expression and enzymatic activity of xenobiotic-metabolizing enzymes and the ability to form bile 
canaliculae [42-44]. Primary human hepatocytes cultures can be set-up in such a way that cells from a single donor are used, or hepatocytes of different donors can be pooled in one single culture. Thus, primary human hepatocytes allow for the study of interindividual differences in response to xenobiotic exposure. However, there are also some limitations to the use of this cell system. First, the lifespan of primary human hepatocytes in culture is limited to several weeks at most [45-47]. Also, primary human hepatocytes are fully differentiated cells and thus will not divide, thereby limiting the amount of available cells to those initially brought into culture. In addition, the availability of these cells depends on the accessibility to human liver tissue, which is rather scarce. Although (cryopreserved) primary human hepatocytes are commercially available, the price tag for these cells is rather high due to their limited availability and inability to divide. Finally, like the carcinoma-derived cell models, primary human hepatocytes only represent one of the cell-types from a whole population of cells residing in the liver. To cover the complete response of the liver to xenobiotic exposure, a model containing as many of the cells represented in the liver as possible would be optimal.

\section{- $\quad$ Precision cut human liver slices}

Precision cut human liver slices, like primary human hepatocytes, are prepared from human liver tissue by the use of specialized slicing equipment to produce slices with a predefined diameter and thickness. Tissue slices can be maintained in cell culture medium in a highly oxygenated surrounding [20]. The precision cut slices allow sufficient supply of nutrients and ample oxygenation to the cells in the inner cell layers [48]. Unlike with the preparation of primary human hepatocytes, where the natural integrity of the liver has to be disturbed in order to isolate the cells, the preparation of tissue slices has no effect on the natural anatomy of the tissue [20]. Precision cut liver slices have been proven useful as an in vitro model to study liver toxicity [49]. Furthermore, this model also allows for the study of interindividual variation with the preparation of precision cut tissue slices from multiple donors. But, as with any in vitro model, also this model comes with some restrictions.

The lifespan of precision cut human liver slices is even more limited than the lifespan of primary human hepatocytes. Assuming optimal culture conditions are provided, the maximal 
time of culture should not exceed 5 days [50]. Thereby, the procedure for the preparation and incubation of these tissue slices is quite labor intensive and requires substantial amounts of lab space [20]. Also this cell model contains tissue with fully differentiated cells that will not allow for further multiplication. Furthermore, the availability of precision cut human liver slices, like primary human hepatocytes, depends on the accessibility to human liver tissue. Although some attempts have been undertaken to cryopreserve precision cut tissue slices, the results were only partially successful [51]. Hence, the limited commercial availability and high costs associated with this in vitro model. Also, due to the lack of an effective preservation method for liver tissue and/or precision cut liver slices the preparation and incubation of precision cut liver slices has to start immediately after human liver tissue comes available. This puts constraints on the ability to plan experiments in time.

- $\quad$ Blood as a reference tissue for human liver

Although the liver is the primary organ of choice to study xenobiotic related responses including toxicity, it is evident that due to its anatomical position, accessibility to this particular organ is limited. As mentioned multiple times above, accessibility to human liver tissue is one of the major restrictions in the usability of in vitro human liver models. Therefore, one has searched for alternative, reference tissues that are easily accessible without the need for invasive procedures to obtain tissue. Whole blood or peripheral (mononuclear) blood cells are examples of such tissues. At any given time large amounts of blood pass through the liver, blood is filtered and compounds are exchanged, as such establishing a constant communication between the liver and blood [52]. Due to this characteristic, researchers have evaluated the option to use blood as a reference or surrogate tissue to monitor liver function, including the response to xenobiotic exposure [53-55]. This model obviously does not allow for the evaluation of the response of individuals to the intentional exposure to harmful xenobiotics due to apparent ethical constraints. Nevertheless, it can be used to monitor the interindividual responses to unintentional exposure to harmful xenobiotics or the response of individuals after exposure to harmless or safe doses of xenobiotics like pharmaceuticals. 
For instance, the latter has been proven to provide new insights in the molecular mechanism related to the responses towards Acetaminophen (a.k.a Paracetamol) exposure [56-60].

A summary of the advantages and disadvantages associated to the models discussed above is provided in Table 1.The main point of interest for this thesis, the ability or inability of a model to evaluate interindividual variation, is emphasized in italic. Of the true human liver-related models, only primary human hepatocytes and precision cut human liver slices allow for this opportunity, making these in vitro systems the preferred models to work with in this thesis.

\begin{tabular}{|l|l|l|}
\hline Model & Advantages & Disadvantages \\
\hline $\begin{array}{l}\text { Carcinoma-based, } \\
\text { immortalized cell lines }\end{array}$ & $\begin{array}{l}\text { - long lifespan } \\
\text { - ability to divide } \\
\text { - easy accessibility to cells } \\
\text { - commercially available at low costs }\end{array}$ & $\begin{array}{l}\text { - lower levels of metabolizing enzymes } \\
\text { - tumorigenic (genetic) background } \\
\text { - single cell-type } \\
\text { - inability to asses interindividual variation }\end{array}$ \\
\hline $\begin{array}{l}\text { Primary human } \\
\text { hepatocytes }\end{array}$ & $\begin{array}{l}\text { - true liver cell-like genetic background } \\
\text { - liver-like levels of metabolizing enzymes } \\
\text { - ability to asses interindividual variation }\end{array}$ & $\begin{array}{l}\text { - limited lifespan (up to several weeks) } \\
\text { - inability to divide } \\
\text { - limited accessibility to cells } \\
\text { - commercially available at high costs } \\
\text { - single cell-type }\end{array}$ \\
\hline $\begin{array}{l}\text { Precision cut human liver } \\
\text { slices }\end{array}$ & $\begin{array}{l}\text { - true liver cell-like genetic background } \\
\text { - liver-like levels of metabolizing enzymes } \\
\text { - ability to asses interindividual variation } \\
\text { - natural integrity of the liver is conserved } \\
\text { - multiple cell-types }\end{array}$ & $\begin{array}{l}\text { - limited lifespan (up to several days) } \\
\text { - inability to divide } \\
\text { - limited accessibility to cells } \\
\text { isolation procedure }\end{array}$ \\
\hline $\begin{array}{l}\text { Blood } \\
\text { (liver reference tissue) }\end{array}$ & $\begin{array}{l}\text { - lack of commercial availability } \\
\text { - low costs } \\
\text { - ability to asses interindividual variation }\end{array}$ & $\begin{array}{l}\text { - no liver-like genetic background } \\
\text { restricted practical applications }\end{array}$ \\
\hline
\end{tabular}

Table 1; Summary of liver models

Overview of advantages and disadvantages associated with human liver in vitro models

\section{Conventional toxicology}

To establish the adverse responses to xenobiotic exposure of a cell or even organ, several parameters enabling the detection of these responses have been developed over the years. Changes to the morphology of an organ can easily be detected by visual inspection, while for the morphological evaluation of cells a microscope can be used. 
The integrity of cellular membranes, which are damaged and become leaky in a toxicity response, can be checked by a trypan blue exclusion assay. Damaged cells will allow for the trypan to enter, and will appear blue when evaluated under the microscope. Undamaged cells stay colorless. Another test that provides information on the integrity of the cellular membrane is the lactate dehydrogenase assay [61]. Lactate dehydrogenase leaks from the cell when the membrane becomes damaged, therefore more lactate dehydrogenase will be detected whit a higher toxicity response.

As mentioned above, some xenobiotics are known to cause damage to the molecular integrity of the DNA-molecule by inducing strand breaks. An assay that can be used to detect these DNAstrand breaks is the COMET-assay, which is based on a single cell gel electrophoresis. In this assay damaged DNA is broken down to short fragments which have a faster migration pattern in the electrophoresis gel than long, undamaged DNA-strands leading to the appearance of a comet-like tail of fragmented DNA, hence the name of the assay. Another assay for the detection of DNA-strand breaks is the $\mathrm{yH} 2 \mathrm{Ax}$ stain [62]. The $\mathrm{\gamma H} 2 \mathrm{Ax}$ stain is based on an antibody based staining, which detects the phosphorylated form of $\mathrm{H} 2 \mathrm{Ax}\left(\mathrm{\gamma H}_{2} \mathrm{Ax}\right)$ which will appear after DNA-strand breakage has occurred.

In addition to the before mentioned general tests for toxicity responses, there are also some tests available that are more specific for the detection of liver toxicity. One example is the assessment of cytochrome P450 (CYP) enzyme activity levels. CYP enzymes are involved in the metabolisms of numerous compounds in the human body, including the Phase I metabolism of many xenobiotics in the liver [63]. Therefore, the level of active CYP enzymes in normal, healthy human liver tissue/cells is generally high. Damaged cells will show lower CYP enzyme levels and are often not able to induce activity of these enzymes after xenobiotic exposure.

Finally, the liver function test is composed of several parameters that can be measured in the blood to evaluate the general state of the liver in vivo. This liver test in general includes, but is not limited to, the measurement of alkaline phosphatase, gamma glutamyl transpeptidase, alanine transaminase, lactate dehydrogenase, total bilirubin and albumin levels [64]. The outcome of these parameters are compared to reference values to determine the severity of liver failure, for instance caused in response through the exposure to a liver toxic xenobiotic. 
The ability of the above mentioned assays to evaluate interindividual variation depends on the in vitro model they are applied to. For instance, the COMET-assay has been used to evaluate interindividual variation in DNA-damage in human mononuclear blood cells in response to the exposure to environmental genotoxic agents [65]. Also, interindividual variation in the enzyme activity of CYP enzymes in human liver tissue $[66,67]$ and primary human hepatocytes [68] has been studied. However all of the above mentioned tests have one main disadvantage in common which is that they can only provide information on (liver) toxicity once pronounced damage has already occurred $[69,70]$. Assays that would be of a more predictive character are therefore desirable. Several of such assays have become available in the recent past and will be discussed further below.

\section{Hepatotoxicity and hepatocarcinogenesis}

Toxicity of the liver, also hepatotoxicity, is a complicated process of which the molecular mechanism of which still is not fully elucidated. In the context of drug development, it is one of the main reasons for withdrawal of drugs from the market (at least $25 \%$ of drugs, [71]). Hepatotoxicity is a process in which the cells of the liver become damaged, leading to cell death, subsequent functional failure of the liver which eventually leads to severe malady or even death. Several mechanisms behind the initiation and aggravation of liver cell damage have been proposed.

One such mechanism affects the flow of bile through the liver. The formation of bile, needed for the digestion of lipids, is a key function of the liver [72]. Incapacity of the liver to produce bile or an obstruction of bile-flow through the liver is a condition named cholestasis [73]. Aberrations in the bile-system are destructive for hepatocytes, leading to apoptosis and as such liver damage. Many drugs are known to have the ability to cause cholestasis [74]. In addition, interindividual variation in the susceptibility towards cholestasis is known to exist, and increasing evidence suggests that genetic factors are causal to this variation $[75,76]$.

Another mechanism through which hepatotoxicity can occur is through the stimulation of a liver-related immune response causing an inflammatory response [77]. During this inflammatory process, the formation of reactive oxygen species (ROS) leads to cellular damage which can lead to apoptosis. Usually, the liver contains a large pool of glutathione which acts as an antioxidant by neutralizing the ROS before 
they can cause any harm. However, several drugs like for instance Acetaminophen are known to reduce the availability of glutathione in the liver till as low as $10 \%$ of normal values [78-80]. During the metabolism of Acetaminophen in the liver by cytochrome P450 enzymes, a reactive metabolite, $\mathrm{N}$-acetyl-p-benzoquinone imine (NAPQI), is formed $[79,80]$. Normally, NAPQI is neutralized by covalent binding to glutathione, however with NAPQI levels that are too high or, the other way around, with glutathione levels that are to low NAPQI can form adducts with proteins, decreasing or disabling their functionality to the cell leading to toxicity $[79,80]$.

Finally, mitochondrial dysfunction is a process that has been indicated to cause hepatotoxicity [77]. As mentioned above, the liver is a very metabolically active organ and therefore has a very high energy demand. Mitochondria are able to generate energy by the tricarboxylic acid cycle, fatty acid oxidation and oxidative phosphorylation and as such are the main source of energy for the cell [81]. Several compounds are known to affect the oxidation of lipids in the mitochondria, leading to an accumulation of lipid vesicles, named steatosis. Due to the steatosis the mitochondria lose functionality and deprive the cell from energy eventually resulting in cell death $[77,81]$. During the generation of energy for the cell, large amounts of ROS are formed in the mitochondria. As described above, ROS especially in high concentrations can cause damage to the mitochondria also reducing its functionality. Furthermore, when ROS from overloaded or damaged mitochondria is released into the cell, it can induce or aggravate the processes described above [82].

All of the above mentioned processes lead to cellular damage in the liver, and if not ceased can cause a progressive deterioration of the liver. A final endpoint in this process is the formation of liver cancer a.k.a. hepatocarcinogenesis. The occurrence of hepatocellular carcinoma is a world-wide issue, since it is the sixth most common cancer and the third leading cause of cancer $[83,84]$. Many causes like chronic hepatitis viral infection, exposure to carcinogenic food-contaminants including Aflatoxin B1 and alcohol consumption have been indicated as factors influencing the development of liver cancer [83-85]. Although the exact mechanisms behind the development of liver cancer are still not fully understood, it has been shown to be a multi-stage process [84]. In most cases persistent damage to the liver, as caused by the processes described above, triggers a response named fibrosis. This response encompasses the formation of excessive amounts of fibrous connective tissue in an attempt to protect further cells from damage 
[83]. However, this response can escalate to the point where there is too much connective tissue, leading to a reduced liver-function and morphological changes to the liver $[83,84]$. This advanced stage of liver fibrosis is also known as liver cirrhosis. Further progression leads to even more severe damage to the liver cells to the point where also DNA-damage occurs, leading to the formation of mutated cells and the subsequent development of cancerous tissue at which the end-stage of liver cancer has been reached $[83,84]$.

Although science progressively contributes to our understanding of the mechanism behind hepatotoxicity and hepatocarcinogenesis, the knowledge of what determines an individual's susceptibility towards these processes is still very rudimental $[85,86]$. Variations in the main metabolizing enzymes of the liver (cytochrome P450) have been proposed as possible candidates [86]. Nevertheless, further factors and multi-factorial approaches within a large population of individuals need to be taken into consideration in order to gain further insight [85].

\section{Toxicogenomics}

Over the last few decades the so called 'omics techniques have become available. These techniques are based on the measurement of not one but many, often thousands of parameters simultaneously. Several different 'omics techniques are available including those for the determination of genes, metabolites and proteins. In the specific case of the simultaneous measurement of genes, this technique is called genomics. The study of genomics towards a toxicity response, for instance induced by the exposure to a xenobiotic is termed toxicogenomics. The 'omics techniques have been claimed to be more sensitive than the above described conventional toxicological assays, facilitating the measurement of responses to xenobiotic exposure before obvious harm has occurred demonstrating their predictive value [87-90]. In addition, since these techniques do not just measure the response of one parameter but summarize the responses of many, many parameters they can provide additional information in the molecular mechanism behind a particular response. Mechanistic understanding of the adverse responses towards xenobiotic exposure could help in defining preventative measures.

Genomics techniques have experienced a boost in their development following the completion of the 'Human genome project' in 2003 [91]. The sequence of 99\% of the human genome is 
now known, enabling the development of a chip-type system to measure the expression of human genes, the microarray [92]. Thus, microarrays can be used to study the expression of genes or in other words the transcription levels of genes, this process is named transcriptomics. In response to xenobiotic exposure the expression levels of mRNA (genes) change, these changes can be evaluated by comparing the mRNA expression levels of exposed samples to control (non-exposed) samples. For instance, samples from individuals taking a certain drug can be compared to samples from individuals that do not take this drug in order to study gene expression responses of individuals towards this particular drug. Also, genomics technique allows for the comparison of responses between individuals, permitting the evaluation of interindividual differences towards xenobiotic exposure. Additionally, the comparison of baseline gene-expression levels (without the intentional exposure to any xenobiotics) shed a light on the normal, biological variation in gene expression levels within the human population. As mentioned before, it is vital to understand the interindividual variation within the human population in order to identify potentially vulnerable individuals and to make a well-reasoned estimation of risk. Thereby, the molecular information provided by genomics can be used to develop a more thorough understanding in the physiological differences between individuals.

\section{Regulation of gene expression}

The central dogma of molecular biology, defined by Francis Crick [93] dictates the flow of genetic information a.k.a. gene expression, through a system; from DNA, to RNA, to protein (see Figure 1). Although this statement is still very much true, additional factors influencing gene expression have been defined over the years. These factors can regulate the expression of genes without changing the genetic code on the DNA and are summarized under the term epigenetics.

One example of an epigenetic mechanism for gene regulation is DNA-methylation [94]. By the addition of methyl-groups to the DNAhelix, the transcription of DNA to mRNA is blocked at this site, thereby stagnating the process towards the formation of proteins (also see Figure 1). Variation in DNA-methylation patterns have been said to cause interindividual differences. For instance, in monozygotic twins who share an identical genetic code, small variations in DNAmethylation levels acquired throughout life could be causal of disease appearance, such as diabetes, in one individual while the other stays 
unaffected [95]. Also, differences in methylation levels of drug-metabolizing genes have been proposed as possible factors explaining the differences in drug responses between individuals [96, 97].

Another example of epigenetic regulation of gene expression is microRNA (miRNA, see Figure 1). miRNA's are short, non-coding RNA molecules of about 22 nucleotides long, that can bind to the sequence of mRNA molecules thereby influencing the conversion-rate of mRNA to protein [98]. The expression of miRNAs can be tissue specific and can be influenced by the exposure to xenobiotics. For instance, after exposure to liver-toxic Acetaminophen doses, miR-122 a liver expressed miRNA is released into circulation [99, 100]. Also miRNAs have been proposed as an additional factor that could explain human interindividual variation in the gene expression response towards xenobiotic exposure. Like for mRNA, chip-based array platforms have been developed for the measurement of genome-wide miRNA expression levels [101] or DNA-methylation status [102]. Together with the information gathered from mRNA, data from miRNA and DNA-methylation arrays could lead to additional insight into the causes of interindividual variation.

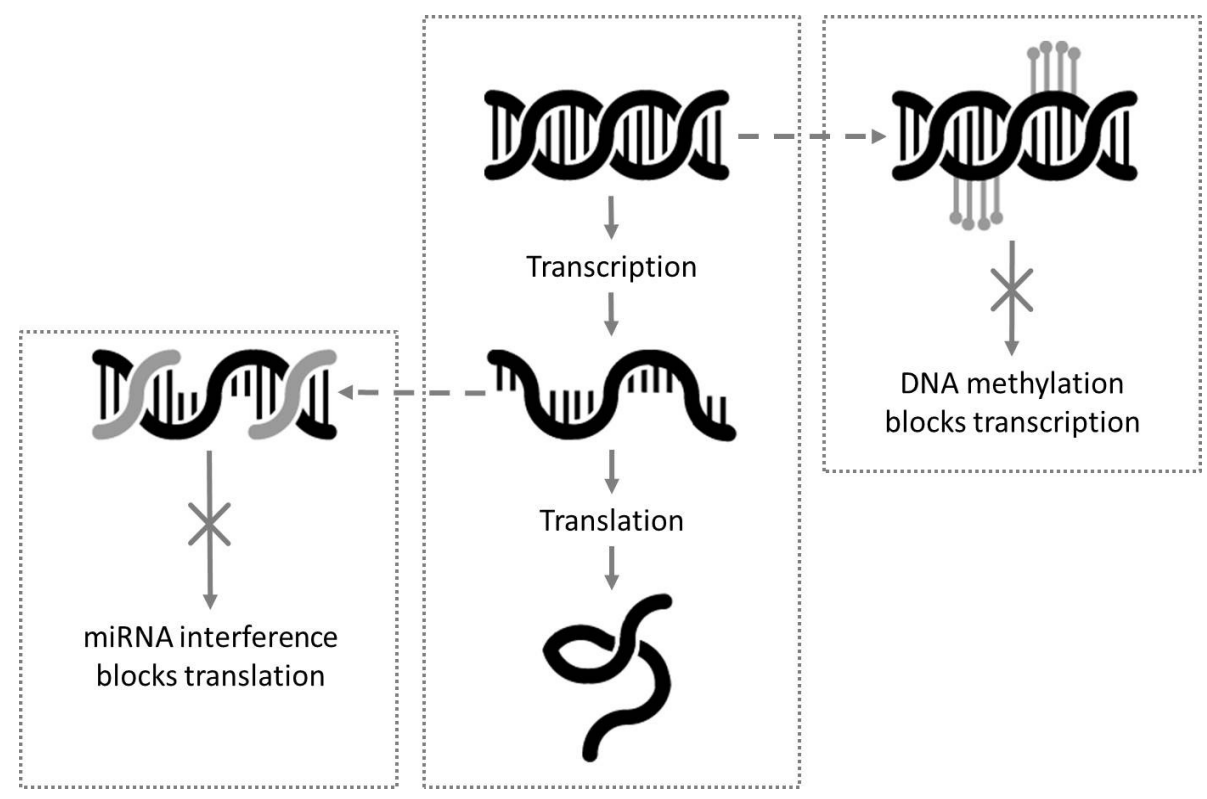

Figure 1; Gene expression

Central dogma of molecular biology (middle frame) dictates the flow of genetic information DNA, to RNA, to protein. Regulatory mechanisms of gene expression are miRNA interference (left frame) and DNA methylation (right frame). 


\section{Model xenobiotics}

In order to evaluate the additional information the above described 'omics techniques can provide on the molecular response to xenobiotic exposure in different cell models and/or individuals, several well-described xenobiotics were used in the research described in this thesis.

- $\quad$ Aflatoxin B1; for short AFB is a naturally occurring mycotoxin, produced by the fungi Aspergillus flavus and Aspergillus paraciticus that occurs in our food chain $[2,103,104]$. AFB requires both activation and deactivation of its reactive metabolite by metabolism through cytochrome P450 enzymes in the liver [105]. The active metabolite of AFB is able to covalently bind to DNA, making it one of the most potent human hepato-carcinogens known.

- $\quad$ Benzo( $\alpha$ )pyrene and 2-Nitrofluorene; respectively BaP and NF are both products formed during the combustion of organic products. They can appear as air pollutants from incomplete combustion of substances such as diesel and gasoline, but are also formed during the preparation of food (especially charred meats) $[2,106-108]$. Both substances are metabolized by the cytochrome P450 system in the liver [108, 109]. Both compounds produce metabolites that can cause DNA-strand brakes through binding to the DNA-strand, making them potent carcinogens.

- $\quad$ Acetaminophen; also known as Paracetamol, for short APAP is the most popular over-the-counter available analgesic worldwide. However, it also is the primary cause of acute liver toxicity in the US and Europe $[110,111]$. APAP causes drug-induced liver injury through the formation of a reactive metabolite (NAPQI) after metabolism by cytochrome P450 [112114]. High levels of NAPQI lead to protein-adduct formation, which in turn causes oxidative stress that finally results in liver damage [115]. 


\section{Aim and outline of this thesis}

The currently available technologies allow for the measurement of genome-wide gene expression levels and gene regulatory mechanisms. We hypothesize that by the analysis of these data we will be able to pinpoint genes/gene sets that predispose for increased susceptibly of an individual upon challenge by xenobiotics.

More and more approaches for data analyses on toxicogenomics (related) data become available, like for instance benchmark dose analyses and Bayesian network analysis. This increases our potential to provide better biological interpretation of these often complicated datasets. Furthermore, by comparing the data from different human individuals with the data available from the currently used human cell models, we gain more insight in the overall applicability and comparability of these cell models to the human situation. To address these research aims, the objective of this thesis is to compare gene expression and gene regulatory mechanisms on an individual level using different statistical approaches, as such evaluating the interindividual variation in the human population. Additionally, the gene expression responses of human individuals, both before and after xenobiotic exposure, are compared to the gene expression responses in some commonly used human cell models.

In Chapter $\mathbf{2}$ an in vitro model based on precision cut human liver slices was used to evaluate the interindividual variation in genome response to exposure to Aflatoxin B1, Benzo( $\alpha$ )pyrene, 2-Nitrofluorene and Acetaminophen (a.k.a. Paracetamol) using a combination of benchmark dose analysis, Spearman correlation analysis and Bayesian network analysis to enable biological interpretation. Chapter $\mathbf{3}$ focused on another in vitro cell model consisting of primary human hepatocytes from several individuals from which gene expression and metabolite responses after Acetaminophen exposure were evaluated for interindividual variation. A comparison between the baseline gene expression patterns of primary human hepatocytes from different individuals and the baseline gene expression patterns of two carcinoma based human cell models (HepG2 and HepaRG) was made in

Chapter 4. Furthermore, in this chapter the gene expression responses from HepG2, HepaRG and primary human hepatocytes after exposure to Aflatoxin B1 and Benzo( $\alpha$ )pyrene were evaluated. In Chapter 5 the correlation between the baseline methylation status of genes and the baseline expression levels of these genes was assessed for different individuals by the use of primary human hepatocytes. In addition, the 
correlation between baseline gene expression level/methylation level and the benchmark dose response after exposure to Aflatoxin B1 and Benzo( $\alpha$ )pyrene was evaluated. The correlation between the gene expression responses and the interindividual variation in mRNA and its regulatory miRNA's and metabolite formation were evaluated for several low/therapeutic-dose Acetaminophen exposures in an in vivo study with several individuals, which was described in Chapter 6. Finally,

Chapter $\mathbf{7}$ contains the summary and discussion on the final outcome of the work presented in this thesis. 


\section{References}

1. Hanninen, O., A. Aitio, and E. Hietanen, Physiological defence against xenobi otics at their portals of entry to the organism. Med Biol, 1979. 57(5): p. 251-5.

2. Miller, J.A. and E.C. Miller, Natural and synthetic chemical carcinogens in the etiology of cancer. Cancer Res, 1965. 25(8): p. 1292-304.

3. Eun, J.W., et al., Discriminating the molecular basis of hepatotoxicity using the large-scale characteristic molecular signatures of toxicants by expression profiling analysis. Toxicology, 2008. 249(2-3): p. 176-83.

4. Kohle, C., M. Schwarz, and K.W. Bock, Promotion of hepatocarcinogenesis in humans and animal models. Arch Toxicol, 2008. 82(9): p. 623-31.

5. Nesnow, S., et al., Lung tumorigenic interactions in strain A/J mice of five environmental polycyclic aromatic hydrocarbons. Environmental health perspectives, 1998. 106 Suppl 6: p. 1337-46.

6. Oliveira, P.A., et al., Chemical carcinogenesis. Anais da Academia Brasileira de Ciencias, 2007. 79(4): p. 593-616.

7. Uetrecht, J. and D.J. Naisbitt, Idiosyncratic adverse drug reactions: current concepts. Pharmacol Rev, 2013. 65(2): p. 779-808.

8. Bozina, N., V. Bradamante, and M. Lovric, Genetic polymorphism of metabolic enzymes P450 (CYP) as a susceptibility factor for drug response, toxicity, and cancer risk. Arhiv za higijenu rada i toksikologiju, 2009. 60(2): p. 217-42.

9. Kola, I. and J. Landis, Can the pharmaceutical industry reduce attrition rates? Nature reviews. Drug discovery, 2004. 3(8): p. 711-5.

10. Omiecinski, C.J., et al., Xenobiotic metabolism, disposition, and regulation by receptors: from biochemical phenomenon to predictors of major toxicities. Toxicol Sci, 2011. 120 Suppl 1: p. S49-75.

11. Urquhart, B.L., R.G. Tirona, and R.B. Kim, Nuclear receptors and the regulation of drug-metabolizing enzymes and drug transporters: implications for interindividual variability in response to drugs. J Clin Pharmacol, 2007. 47(5): p. 566-78.

12. Yang, L., et al., Gene expression variability in human hepatic drug metabolizing enzymes and transporters. PLoS One, 2013. 8(4): p. e60368.

13. European-Commission, Opinion of the Scientific Committee on Toxicity, Eco toxicity and the Environment on The BUAV-European Coalition to End Animal Experiments Report: The Way Forward - Action to End Animal Toxicity Testing, 2004.

14. The-Staff-of-ACSH, Of Mice and Mandates: Animal Experiments, Human Cancer Risk, and Regulatory Policies, 1997.

15. Levy, N., The use of animal as models: ethical considerations. International journal of stroke : official journal of the International Stroke Society, 2012. 7(5): p. 440-2.

16. Lehman, A.J. and O.G. Fitzhugh, 100-fold margin of safety. Assoc. Food Drug Off. U.S.Q. Bull., 1954. 18: p. 33-35.

17. Dorne, J.L.C.M. and A.G. Renwick, The Refinement of Uncertainty/Safety Factors in Risk Assessment by the Incorporation of Data on Toxicokinetic Variability in Humans. Toxicological Sciences, 2005. 86(1): p. 20-26.

18. KEMI-Karolinska-Institute-Sweden. HUMAN HEALTH RISK ASSESSMENT Proposals for the use of assessment (uncertainty) factors; Application to risk assessment for plant protection products, industrial chemicals and biocidal products within the European Union. 2003; Available from: http://www.kemi. se/Documents/Publikationer/Trycksaker/Rapporter/Rapport1_03.pdf.

19. Fasinu, P., P.J. Bouic, and B. Rosenkranz, Liver-based in vitro technologies for 
drug biotransformation studies - a review. Curr Drug Metab, 2012. 13(2): p. 215-24.

20. de Graaf, I.A., et al., Preparation and incubation of precision-cut liver and intestinal slices for application in drug metabolism and toxicity studies. Nature protocols, 2010. 5(9): p. 1540-51.

21. Kmiec, Z., Cooperation of liver cells in health and disease. Adv Anat Embryol Cell Biol, 2001. 161: p. III-XIII, 1-151.

22. Treyer, A. and A. Musch, Hepatocyte polarity. Compr Physiol, 2013. 3(1): p. 243-87.

23. Knowles, B.B., C.C. Howe, and D.P. Aden, Human hepatocellular carcinoma cell lines secrete the major plasma proteins and hepatitis B surface antigen. Science, 1980. 209(4455): p. 497-9.

24. Morris, K.M., et al., Complement biosynthesis by the human hepatoma-derived cell line HepG2. J Clin Invest, 1982. 70(4): p. 906-13.

25. Aninat, C., et al., Expression of cytochromes P450, conjugating enzymes and nuclear receptors in human hepatoma HepaRG cells. Drug Metab Dispos, 2006. 34(1): p. 75-83.

26. Cerec, V., et al., Transdifferentiation of hepatocyte-like cells from the human hepatoma HepaRG cell line through bipotent progenitor. Hepatology, 2007. 45(4): p. 957-67.

27. Guillouzo, A., et al., The human hepatoma HepaRG cells: a highly differentiated model for studies of liver metabolism and toxicity of xenobiotics. Chem Biol Interact, 2007. 168(1): p. 66-73.

28. Jennen, D.G., et al., Comparison of HepG2 and HepaRG by whole-genome gene expression analysis for the purpose of chemical hazard identification. Toxicol Sci, 2010. 115(1): p. 66-79.

29. Song, M.K., et al., Identification of molecular signatures predicting the carcinogenicity of polycyclic aromatic hydrocarbons (PAHs). Toxicol Lett, 2012. 212(1): p. 18-28.

30. van Delft, J.H., et al., Discrimination of genotoxic from non-genotoxic carcinogens by gene expression profiling. Carcinogenesis, 2004. 25(7): p. 1265-76.

31. Westerink, W.M. and W.G. Schoonen, Cytochrome P450 enzyme levels in HepG2 cells and cryopreserved primary human hepatocytes and their induction in HepG2 cells. Toxicol In Vitro, 2007. 21(8): p. 1581-91.

32. Westerink, W.M. and W.G. Schoonen, Phase II enzyme levels in HepG2 cells and cryopreserved primary human hepatocytes and their induction in HepG2 cells. Toxicol In Vitro, 2007. 21(8): p. 1592-602.

33. Guo, L., et al., Similarities and differences in the expression of drugmetabolizing enzymes between human hepatic cell lines and primary human hepatocytes. Drug Metab Dispos, 2011. 39(3): p. 528-38.

34. Jennen, D.G., et al., Biotransformation pathway maps in WikiPathways enable direct visualization of drug metabolism related expression changes. Drug Discov Today, 2010. 15(19-20): p. 851-8.

35. Olsavsky, K.M., et al., Gene expression profiling and differentiation assessment in primary human hepatocyte cultures, established hepatoma cell lines, and human liver tissues. Toxicol Appl Pharmacol, 2007. 222(1): p. 42-56.

36. Wilkening, S., F. Stahl, and A. Bader, Comparison of primary human hepato cytes and hepatoma cell line Hepg2 with regard to their biotransformation properties. Drug Metab Dispos, 2003. 31(8): p. 1035-42.

37. Kanebratt, K.P. and T.B. Andersson, Evaluation of HepaRG cells as an in vitro model for human drug metabolism studies. Drug Metab Dispos, 2008. 36(7): 
38. Kanebratt, K.P. and T.B. Andersson, HepaRG cells as an in vitro model for evaluation of cytochrome P450 induction in humans. Drug Metab Dispos, 2008. 36(1): p. 137-45.

39. Lambert, C.B., et al., Reproducible chemical-induced changes in gene expression profiles in human hepatoma HepaRG cells under various experimental conditions. Toxicol In Vitro, 2009. 23(3): p. 466-75.

40. Turpeinen, M., et al., Functional expression, inhibition and induction of CYP enzymes in HepaRG cells. Toxicol In Vitro, 2009. 23(4): p. 748-53.

41. Hewitt, N.J., et al., Primary hepatocytes: current understanding of the regulation of metabolic enzymes and transporter proteins, and pharmaceutical practice for the use of hepatocytes in metabolism, enzyme induction, transporter, clearance, and hepatotoxicity studies. Drug metabolism reviews, 2007. 39(1): p. 159-234.

42. Godoy, P., et al., Recent advances in 2D and 3D in vitro systems using primary hepatocytes, alternative hepatocyte sources and non-parenchymal liver cells and their use in investigating mechanisms of hepatotoxicity, cell signaling and ADME. Archives of toxicology, 2013. 87(8): p. 1315-530.

43. LeCluyse, E.L., et al., Organotypic liver culture models: meeting current challenges in toxicity testing. Critical reviews in toxicology, 2012. 42(6): p. 501-48.

44. Swift, B., N.D. Pfeifer, and K.L. Brouwer, Sandwich-cultured hepatocytes: an in vitro model to evaluate hepatobiliary transporter-based drug interactions and hepatotoxicity. Drug metabolism reviews, 2010. 42(3): p. 446-71.

45. Dunn, J.C.Y., R.G. Tompkins, and M.L. Yarmush, Long-Term in Vitro Function of Adult Hepatocytes in a Collagen Sandwich Configuration. Biotechnology Progress, 1991. 7(3): p. 237-245.

46. LeCluyse, E.L., K.L. Audus, and J.H. Hochman, Formation of extensive canalicular networks by rat hepatocytes cultured in collagen-sandwich configuration. Am J Physiol, 1994. 266(6 Pt 1): p. C1764-74.

47. Michalopoulos, G.K., et al., Comparative analysis of mitogenic and morpho genic effects of HGF and EGF on rat and human hepatocytes maintained in collagen gels. J Cell Physiol, 1993. 156(3): p. 443-52.

48. Smith, P.F., et al., Precision cut liver slices: a new in vitro tool in toxicology From: In vitro toxicology: model systems and methods, C.A. McQueen, Editor 1989.

49. Vickers, A.E. and R.L. Fisher, Organ slices for the evaluation of human drug toxicity. Chem Biol Interact, 2004. 150(1): p. 87-96.

50. Vickers, A.E., et al., Organ slice viability extended for pathway characterization: an in vitro model to investigate fibrosis. Toxicol Sci, 2004. 82(2): p. 534-44.

51. Fahy, G.M., et al., Cryopreservation of precision-cut tissue slices. Xenobiotica, 2013. 43(1): p. 113-32.

52. Richerds, I.S. and M. Bourgeois, Principles and practice of toxicology in public health - second edition, ed. J.B. Learning2014: Jones \& Bartlett Learning.

53. Liew, C.-C., et al., The peripheral blood transcriptome dynamically reflects system wide biology: a potential diagnostic tool. Journal of Laboratory and Clinical Medicine, 2006. 147(3): p. 126-132.

54. Mohr, S. and C.-C. Liew, The peripheral-blood transcriptome: new insights into disease and risk assessment. Trends in Molecular Medicine, 2007. 13(10): p. $422-432$.

55. Rockett, J.C., et al., Surrogate tissue analysis: monitoring toxicant exposure and health status of inaccessible tissues through the analysis of accessible tissues 
and cells. Toxicology and applied pharmacology, 2004. 194(2): p. 189-99.

56. Bushel, P.R., et al., Blood gene expression signatures predict exposure levels. Proc Natl Acad Sci U S A, 2007. 104(46): p. 18211-6.

57. Fannin, R.D., et al., Acetaminophen dosing of humans results in blood transcriptome and metabolome changes consistent with impaired oxidative phosphorylation. Hepatology, 2010. 51(1): p. 227-36.

58. Heinloth, A.N., et al., Gene expression analysis offers unique advantages to histopathology in liver biopsy evaluations. Toxicol Pathol, 2007. 35(2): p. 276-83.

59. Huang, J., et al., Genomic indicators in the blood predict drug-induced liver injury. Pharmacogenomics J, 2010. 10(4): p. 267-77.

60. Umbright, C., et al., Blood gene expression markers to detect and distinguish target organ toxicity. Mol Cell Biochem, 2010. 335(1-2): p. 223-34.

61. Decker, T. and M.L. Lohmann-Matthes, A quick and simple method for the quantitation of lactate dehydrogenase release in measurements of cellular cytotoxicity and tumor necrosis factor (TNF) activity. J Immunol Methods, 1988. 115(1): p. 61-9.

62. Rogakou, E.P., et al., Megabase chromatin domains involved in DNA double-strand breaks in vivo. J Cell Biol, 1999. 146(5): p. 905-16.

63. Nishimura, M., et al., Tissue distribution of mRNA expression of human cytochrome P450 isoforms assessed by high-sensitivity real-time reverse transcription PCR. Yakugaku Zasshi, 2003. 123(5): p. 369-75.

64. Limdi, J.K. and G.M. Hyde, Evaluation of abnormal liver function tests. Postgraduate medical journal, 2003. 79(932): p. 307-12.

65. Moller, P., Genotoxicity of environmental agents assessed by the alkaline comet assay. Basic Clin Pharmacol Toxicol, 2005. 96 Suppl 1: p. 1-42.

66. Powell, H., et al., Expression of cytochrome P4502E1 in human liver: assessment by mRNA, genotype and phenotype. Pharmacogenetics, 1998. 8(5): p. 411-21.

67. Rodriguez-Antona, C., et al., Cytochrome P-450 mRNA expression in human liver and its relationship with enzyme activity. Arch Biochem Biophys, 2001. 393(2): p. 308-15.

68. Rhodes, S.P., et al., Simultaneous assessment of cytochrome P450 activity in cultured human hepatocytes for compound-mediated induction of CYP3A4, CYP2B6, and CYP1A2. J Pharmacol Toxicol Methods, 2011. 63(3): p. 223-6.

69. Watkins, P.B., Biomarkers for the diagnosis and management of drug-induced liver injury. Semin Liver Dis, 2009. 29(4): p. 393-9.

70. Watkins, P.B., et al., Aminotransferase elevations in healthy adults receiving 4 grams of acetaminophen daily: a randomized controlled trial. JAMA, 2006. 296(1): p. 87-93.

71. Schuster, D., C. Laggner, and T. Langer, Why drugs fail--a study on side effects in new chemical entities. Current pharmaceutical design, 2005. 11(27): p. 3545-59.

72. Badley, B.W., Bile salts. Can Med Assoc J, 1970. 102(2): p. 159-64.

73. Patel, T., et al., Dysregulation of apoptosis as a mechanism of liver disease: an overview. Seminars in liver disease, 1998. 18(2): p. 105-14.

74. Padda, M.S., et al., Drug-induced cholestasis. Hepatology, 2011. 53(4): p. 1377-87.

75. Bohan, A. and J.L. Boyer, Mechanisms of hepatic transport of drugs: implications for cholestatic drug reactions. Seminars in liver disease, 2002. 22(2): p. 123-36.

76. Pauli-Magnus, C. and P.J. Meier, Hepatobiliary transporters and drug-induced 
cholestasis. Hepatology, 2006. 44(4): p. 778-87.

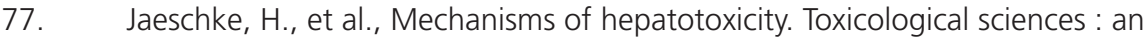
official journal of the Society of Toxicology, 2002. 65(2): p. 166-76.

78. Hinson, J.A., D.W. Roberts, and L.P. James, Mechanisms of acetaminopheninduced liver necrosis. Handb Exp Pharmacol, 2010(196): p. 369-405.

79. Mitchell, J.R., et al., Acetaminophen-induced hepatic necrosis. I. Role of drug metabolism. The Journal of pharmacology and experimental therapeutics, 1973. 187(1): p. 185-94.

80. Mitchell, J.R., et al., Acetaminophen-induced hepatic necrosis. IV. Protective role of glutathione. The Journal of pharmacology and experimental therapeutics, 1973. 187(1): p. 211-7.

81. Pessayre, D., et al., Hepatotoxicity due to mitochondrial dysfunction. Cell biology and toxicology, 1999. 15(6): p. 367-73.

82. Gutterman, D.D., Mitochondria and reactive oxygen species: an evolution in function. Circulation research, 2005. 97(4): p. 302-4.

83. Wallace, M.C. and S.L. Friedman, Hepatic fibrosis and the microenvironment: fertile soil for hepatocellular carcinoma development. Gene expression, 2014. 16(2): p. 77-84.

84. Wong, C.-M. and I.O.L. Ng, Molecular pathogenesis of hepatocellular carcinoma. Liver International, 2008. 28(2): p. 160-174.

85. Macdonald, G.A., Pathogenesis of hepatocellular carcinoma. Clin Liver Dis, 2001. 5(1): p. 69-85.

86. Guengerich, F.P., Polymorphism of cytochrome P-450 in humans. Trends Pharmacol Sci, 1989. 10(3): p. 107-9.

87. Beger, R.D., J. Sun, and L.K. Schnackenberg, Metabolomics approaches for discovering biomarkers of drug-induced hepatotoxicity and nephrotoxicity. Toxicol Appl Pharmacol, 2010. 243(2): p. 154-66.

88. Harrill, A.H. and I. Rusyn, Systems biology and functional genomics approaches for the identification of cellular responses to drug toxicity. Expert Opin Drug Metab Toxicol, 2008. 4(11): p. 1379-89.

89. Vinayavekhin, N., E.A. Homan, and A. Saghatelian, Exploring disease through metabolomics. ACS Chem Biol, 2010. 5(1): p. 91-103.

90. Vlaanderen, J., et al., Application of OMICS technologies in occupational and environmental health research; current status and projections. Occup Environ Med, 2010. 67(2): p. 136-43.

91. International-Human-Genome-Sequencing-Consortium., Finishing the euchromatic sequence of the human genome. Nature, 2004. 431(7011): p. 931-45.

92. Schena, M., et al., Quantitative monitoring of gene expression patterns with a complementary DNA microarray. Science, 1995. 270(5235): p. 467-70.

93. Crick, F., Central dogma of molecular biology. Nature, 1970. 227(5258): p. 561-3.

94. Doerfler, W., DNA methylation--a regulatory signal in eukaryotic gene expression. J Gen Virol, 1981. 57(Pt 1): p. 1-20.

95. Steves, C.J., T.D. Spector, and S.H. Jackson, Ageing, genes, environment and epigenetics: what twin studies tell us now, and in the future. Age Ageing, 2012. 41(5): p. 581-6.

96. Kacevska, M., M. Ivanov, and M. Ingelman-Sundberg, Epigenetic-dependent regulation of drug transport and metabolism: an update. Pharmacogenomics, 2012. 13(12): p. 1373-85.

97. Sim, S.C., M. Kacevska, and M. Ingelman-Sundberg, Pharmacogenomics of drug-metabolizing enzymes: a recent update on clinical implications and 
endogenous effects. The pharmacogenomics journal, 2013. 13(1): p. 1-11.

98. Chen, K. and N. Rajewsky, The evolution of gene regulation by transcription factors and microRNAs. Nat Rev Genet, 2007. 8(2): p. 93-103.

99. Starkey Lewis, P.J., et al., Circulating microRNAs as potential markers of human drug-induced liver injury. Hepatology, 2011. 54(5): p. 1767-1776.

100. Starkey Lewis, P.J., et al., Serum microRNA biomarkers for drug-induced liver injury. Clin Pharmacol Ther, 2012. 92(3): p. 291-3.

101. Liang, Y., et al., Characterization of microRNA expression profiles in normal human tissues. BMC Genomics, 2007. 8: p. 166.

102. Laird, P.W., The power and the promise of DNA methylation markers. Nature reviews. Cancer, 2003. 3(4): p. 253-66.

103. Cotty, P.J., et al., Variation in polygalacturonase production among Aspergillus flavus isolates. Applied and environmental microbiology, 1990. 56(12): p. 3885-7.

104. Horn, B.W. and J.W. Dorner, Regional differences in production of aflatoxin B1 and cyclopiazonic acid by soil isolates of aspergillus flavus along a transect within the United States. Applied and environmental microbiology, 1999. 65(4): p. 1444-9.

105. Wild, C.P., et al., Aflatoxin B1 binding to plasma albumin and liver DNA upon chronic administration to rats. Carcinogenesis, 1986. 7(6): p. 853-8.

106. Lawther, P.J. and R.E. Waller, Coal fires, industrial emissions and motor vehicles as sources of environmental carcinogens. IARC Sci Publ, 1976(13): p. 27-40.

107. Phillips, D.H., Polycyclic aromatic hydrocarbons in the diet. Mutat Res, 1999. 443(1-2): p. 139-47.

108. Beije, B. and L. Moller, 2-Nitrofluorene and related compounds: prevalence and biological effects. Mutat Res, 1988. 196(2): p. 177-209.

109. Zhou, S.F., J.P. Liu, and B. Chowbay, Polymorphism of human cytochrome P450 enzymes and its clinical impact. Drug metabolism reviews, 2009. 41(2): p. 89-295.

110. Kuehn, B.M., FDA focuses on drugs and liver damage: labeling and other changes for acetaminophen. JAMA, 2009. 302(4): p. 369-71.

111. Lee, W.M., Acetaminophen toxicity: changing perceptions on a social/medical issue. Hepatology, 2007. 46(4): p. 966-70.

112. Chen, C., et al., Identification of novel toxicity-associated metabolites by metabolomics and mass isotopomer analysis of acetaminophen metabolism in wild-type and Cyp2e1-null mice. J Biol Chem, 2008. 283(8): p. 4543-59.

113. Chen, C., et al., Serum metabolomics reveals irreversible inhibition of fatty acid beta-oxidation through the suppression of PPARalpha activation as a con tributing mechanism of acetaminophen-induced hepatotoxicity. Chem Res Toxicol, 2009. 22(4): p. 699-707.

114. Daykin, C.A., et al., The comparison of plasma deproteinization methods for the detection of low-molecular-weight metabolites by (1) $\mathrm{H}$ nuclear magnetic resonance spectroscopy. Anal Biochem, 2002. 304(2): p. 220-30.

115. Dahlin, D.C., et al., N-acetyl-p-benzoquinone imine: a cytochrome P-450mediated oxidation product of acetaminophen. Proc Natl Acad Sci U S A, 1984. 81(5): p. 1327-31. 


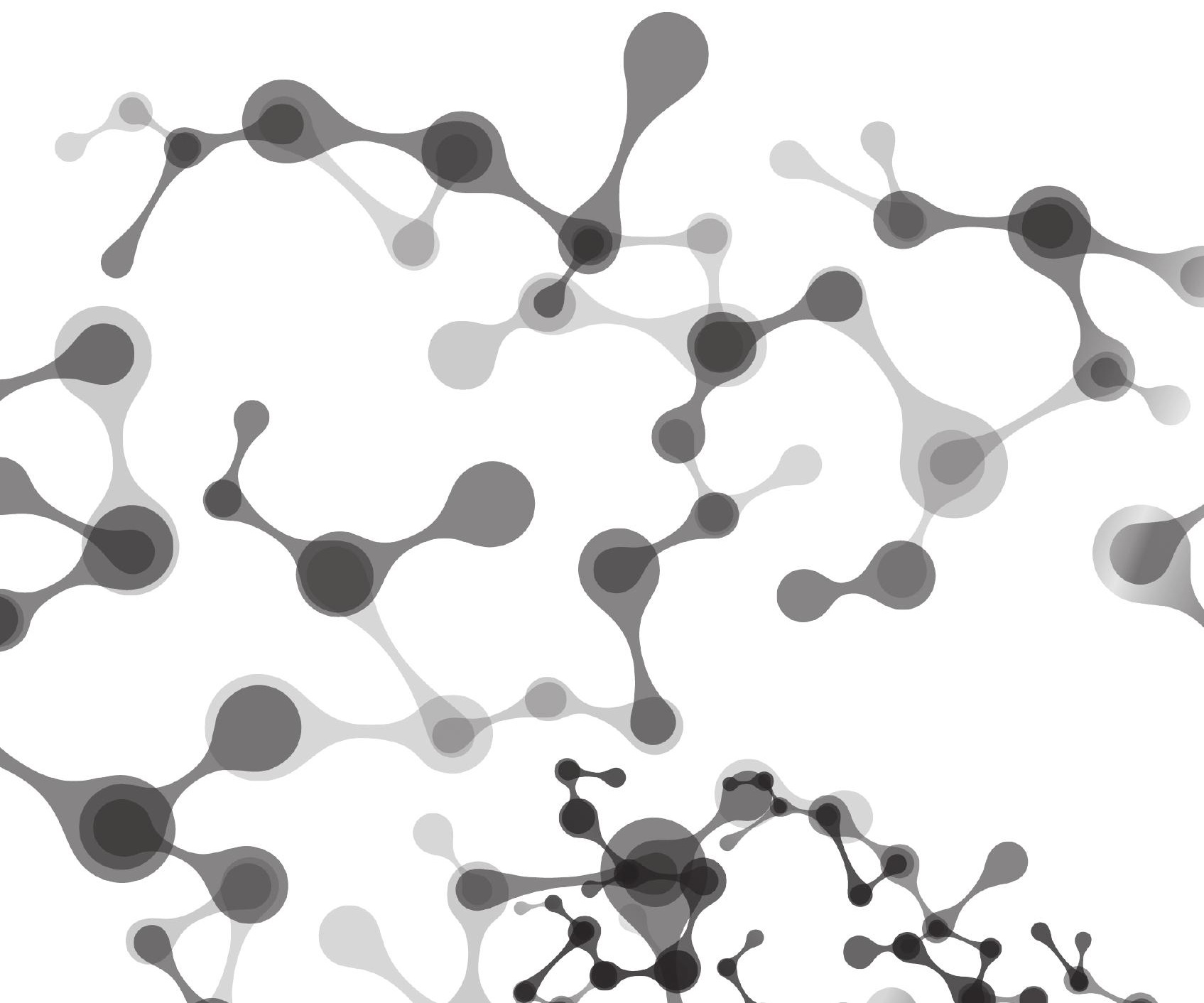




\section{Chapter 2}

Interindividual variation in

response to xenobiotic exposure established in precision-cut human liver slices

Marlon J.A. Jetten

Sandra M. Claessen

Cornelis H.C. Dejong

Agustin Lahoz

José V. Castell

Joost H.M. van Delft

Jos C.S. Kleinjans

Published: Toxicology: 2014 sep 2;232:61-9 


\section{Abstract}

Large differences in toxicity responses occur within the human population. In this study we evaluate whether interindividual variation in baseline enzyme activity (EA)/gene expression (GE) levels in liver predispose for the variation in toxicity responses by assessing doseresponse relationships for several prototypical hepatotoxicants.

Baseline levels of cytochrome-P450 (CYP) GE/EA were measured in precision-cut human liver slices. Slices ( $n=4-5 /$ compound) were exposed to a dose-range of acetaminophen, aflatoxin B1, benzo( $\alpha)$ pyrene or 2-nitrofluorene. Interindividual variation in induced genotoxicity (COMET-assay and CDKN1A/p21 GE) and cytotoxicity (lactate dehydrogenase-leakage), combined with NQO1- and GSTM1induced GE-responses for oxidative stress and GE-responses of several CYPs was evaluated. The benchmark dose-approach was applied as a tool to model exposure responses on an individual level.

Variation in baseline CYP levels, both GE and EA, can explain variation in compound exposure-responses on an individual level. Network analyses enable the definition of key parameters influencing interindividual variation after compound exposure. For 2-nitrofluorene, this analysis suggests involvement of CYP1B1 in the metabolism of this compound, which represents a novel finding. In this study, GSTM1 which is known to be highly polymorphic within the human population, but so far could not be linked to toxicity in acetaminophen-poisoned patients, is suggested to cause interindividual variability in acetaminophen-metabolism, dependent on the individual's gene expression-responses of CYP-enzymes.

This study demonstrates that using interindividual variation within network modelling provides a source for the definition of essential and even new parameters involved in compound-related metabolism. This information might enable ways to make more quantitative estimates of human risks. 


\section{Introduction}

The liver is the main organ involved in the metabolism of xenobiotics and is most often affected by toxicity induced by reactive metabolites [1]. From the total number of metabolic reactions in a liver, about $75 \%$ is accounted for by cytochrome P450 (CYP) enzymes [2] which are involved in the detoxification and formation of toxic metabolites during the metabolism of xenobiotics [3].

CYP1A1 in vivo liver mRNA levels are known to vary 20-130fold between individuals [4-7], while a 21-fold interindividual variation of CYP1A1 enzyme activity (EA) has been reported [5]. For CYP1A2 in vivo liver mRNA-levels, a variation ranging 14-500-fold is known to exist between individuals [4-7]. Interindividual variation in CYP1A2 in vivo EA has been reported to range 8-12-fold [5]. In cultured primary human hepatocytes $(\mathrm{PHH})$, interindividual variation of in mRNA expression levels and EA-levels of CYP1A2 has been demonstrated to be about 4-fold and 2-fold respectively [8], while variation in protein content ranges from 3-51-fold [2, 9-12]. For CYP1B1, in vivo mRNA levels vary 16-fold [4]. For CYP2E1 in vivo an interindividual variation of 6-18-fold regarding mRNA expression level, 4-12-fold concerning EA-level and 2-fold for liver protein content is known $[5,13]$. CYP3A4 is known to have a 64-377-fold interindividual variability in liver mRNA levels [5, 7, 14], with a lower than 5-fold variation in EA [5] and an over 70-fold variation in liver protein content [15]. For CYP3A4, interindividual variation ranges 12-20-fold for mRNA expression levels in $\mathrm{PHH}[8,16]$. EA levels vary 3-fold in $\mathrm{PHH}$ [8], and 40-fold in liver S9 fraction [9]. Protein levels show variation ranging from 3-27-fold in liver microsomes $[10-12,17]$ to $354-$ fold in liver 59 fractions [9]. The above described data are provided with some additional information in supplementary Table 1.

Such interindividual variations in base-line drug-metabolizing enzyme expression/activity have been linked to differences in responses to drugs, toxicants and carcinogens in the human population [18]. Within this context, in this study, we aim at evaluating interindividual variation in precision cut liver slices from multiple human donors. Precision cut liver slices represent an in vitro model that preserves the normal tissue integrity of the liver with all cell types in their natural conformation [19]. In order to evaluate baseline interindividual variation of CYP activities involved in compound metabolism, baseline GE levels and endogenous EA were measured using RT-PCR and substrate turnover-rate, respectively. To evaluate 
variation in compound-induced responses, slices were exposed to multiple doses of acetaminophen (APAP), aflatoxin B1 (AFB), benzo $(\alpha)$ pyrene $(\mathrm{BaP})$ or 2-nitrofluorene $(\mathrm{NF})$. APAP is the most commonly used analgesic drug world-wide, which causes drug-induced liver injury [20]. AFB and BaP are genotoxic carcinogens, while NF is a suspected genotoxic carcinogen in humans. All compounds need CYP-mediated metabolic activation to cause genotoxicity/cytotoxicity. DNA-damage was evaluated using the COMET-assay and cytotoxicity was measured as lactate dehydrogenase (LDH) leakage. Also, GE responses of several CYPs, as well as of genes known to be involved in the cellular stress response (NQO1 and GSTM1) and of a gene particularly involved in the p53-mediated DNA-damage response (CDKN1A/p21) were evaluated.

Secondly, we hypothesize that key parameters causing variability in toxicity responses can be defined by modelling compound-specific response networks using the interindividual variation in measured parameters at both baseline and compound-induced levels to do so. The benchmark dose (BMD) approach, in which BMDs represent dose-response relationships, was applied thus enabling a uniform output measure for all mentioned response parameters for each individual separately. BMDs from several genotoxicity tests have been shown to positively correlate to BMDs calculated from matched-tissue carcinogenicity scores, proving that BMDs can be used to illustrate a link between different response parameters [21]. Finally, compound-specific response networks were generated by combining classical Spearman's correlation and the Bayesian network approach.

\section{Materials and Methods}

\section{Human liver tissue}

Healthy, human liver tissue was obtained from material surplus of patients at the University Hospital Maastricht, the Netherlands. All patients had to undergo partial hepatectomy for colorectal metastasis in the liver. None of the patients received any therapy before hepatectomy that would be expected to affect liver functions. An overview of the donor demographics can be found in supplementary Table 2 . The tumor removal procedure has been described before by Dejong et al [22]. Written informed consent was obtained and the research protocols were approved by the medical ethical committee of Maastricht University Medical Centre and found to be in compliance with the Declaration of Helsinki. All tissue was kept on ice for a maximum of 1 hour in 
oxygenated Krebs-Henseleit buffer (K3753, Sigma-Aldrich, Zwijndrecht, the Netherlands) pH7.4 substituted with sodium bicarbonate $(25 \mathrm{mM}$, CAS no. 144-55-8), calcium chloride (2.5 mM, CAS no. 10043-52-4), glucose (25 mM, CAS no. 50-99-7) and HEPES (10 mM, CAS no. 736545-9) all purchased from Sigma-Aldrich, Zwijndrecht, the Netherlands.

\section{Precision-cut liver slices}

Cylinders with a $4 \mathrm{~mm}$ diameter were prepared from the liver samples with a motor-driven tissue coring tool (Alabama Research and Development, Munford Alabama). From the cylinders, tissue slices of about $100 \mu \mathrm{m}$ thick were prepared using a Krumdieck tissue slicer (Alabama Research and Development, Munford Alabama) filled with ice cold Krebs-Henseleit buffer. A more comprehensive description of the used method is available from De Graaf et al [19].

\section{Incubating precision-cut liver slices}

Williams' medium E with L-glutamine (Sigma-Aldrich, Zwijndrecht, the Netherlands) supplemented with $25 \mathrm{mM}$ glucose (CAS no. 50-99-7) and $50 \mu \mathrm{g} / \mathrm{mL}$ gentamicin (CAS no. 1405-41-0, Invitrogen, Bleiswijk, the Netherlands) was used as culture medium. Slices were placed in a 12-well culture plate (1 slice/well) filled with $1.3 \mathrm{~mL}$ pre-warmed medium/well. All incubations were performed in an airtight culture-chamber flushed with carbogen (95\% O2/5\% CO2), placed on a rotary shaker platform $(90 \mathrm{rpm})$ at $37^{\circ} \mathrm{C}$. Slices were pre-incubated for 1 hour without any compounds, after which slices were transferred to medium containing compounds (also see De Graaf et al [19]). Compounds; acetaminophen (APAP, 2.5, 5 and $10 \mathrm{mM}$, CAS no. 10390-2), aflatoxin B1 (AFB, 2.5, 5, 10, 20, 40 and $80 \mu \mathrm{M}$, CAS no. 116265-8), benzo( $\alpha$ )pyrene (BaP, 5, 10, 20, 40 and $80 \mu \mathrm{M}, \mathrm{CAS}$ no. 50-32-8) and 2-nitrofluorene (NF, 2.5, 5, 10, 20, 40 and $80 \mu \mathrm{M}$, CAS no. 60757-8), all purchased from Sigma-Aldrich, Zwijndrecht, the Netherlands. Doses of the compounds were based on human studies available from literature and range from non-toxic ( $<20 \%$ of total lactate dehydrogenase (LDH) leakage) to toxic ( $>20 \%$ of total LDH leakage). AFB, BaP and NF were dissolved in dimethyl sulfoxide (DMSO, 0.5\% in culture medium, CAS no. 67-68-5, Sigma-Aldrich Zwijndrecht, the Netherlands). All incubations were performed in triplicate using slices of 4 (NF) to 5 (AFB, APAP and BaP) donors. A $24 \mathrm{~h}$ incubation period was chosen based on prior experience showing that incubation periods $>12 \mathrm{~h}$ are needed to induce distinct gene expression changes by 
genotoxic compounds and that the exposure period required for ensuring adequate metabolism of genotoxic compounds was between 12 and 48 h [23-25].

\section{Preparation of slices for the COMET assay}

The method used was adjusted from Plazar et al [26]. In short, a carcinogen-exposed liver slice was positioned on a drop $(160 \mu \mathrm{L})$ of $1 \%$ low melting point (LMP) agarose $\left(37^{\circ} \mathrm{C}\right.$, product code 16520100 , Invitrogen, Blijswijk, the Netherlands), in the middle of a microscope slide, pre-coated with a layer of $1 \%$ normal melting point agarose (product code 16500500, Invitrogen, Blijswijk, the Netherlands). Once the LMP covered the slice, a cover slip was placed. Then, the slice was squashed by gently pressing the cover slip. Subsequently, the slides were kept at $4^{\circ} \mathrm{C}$ for $5 \mathrm{~min}$. to solidify the agarose. Afterwards, the cover slips were removed and another layer of $1 \%$ LMP agarose $\left(37^{\circ} \mathrm{C}\right)$ was added, covered with a cover slip and left to solidify $\left(5 \mathrm{~min} ., 4^{\circ} \mathrm{C}\right)$.

\section{The COMET assay}

The COMET assay was performed as described before by Langie et al with minor modifications [27]. In short, cover slips were removed from the prepared slides after which they were submerged in cold lysis solution (2.5 M sodium chloride (CAS no. 7647-14-5), $0.1 \mathrm{M}$ ethylenediaminetetraacetic acid (EDTA, CAS no 60-00-4), 0.01 $M$ Tris(hydroxymethyl)aminomethane (Tris, CAS no. 77-86-1), 0.25 M sodium hydroxide ( $\mathrm{NaOH}, \mathrm{pH} 10$, CAS no. 1310-73-2) with $10 \%$ DMSO and $1 \%$ polyethylene glycol octylphenol ether (Triton X-100, CAS no. 9002-93-1), all purchased from Sigma-Aldrich, Zwijndrecht, the Netherlands) for $1 \mathrm{~h}$ at $4^{\circ} \mathrm{C}$. Afterwards, slides were rinsed by using phosphate-buffered saline (PBS, Sigma-Aldrich, Zwijndrecht, the Netherlands) and placed in a cooled electrophoreses tank $\left(4^{\circ} \mathrm{C}\right)$ filled with cold electrophoreses buffer $(300 \mathrm{mM} \mathrm{NaOH}$ and $1 \mathrm{mM}$ EDTA, pH 13) for 20 min. to allow DNA unwinding. Then slides were electrophoresed for $20 \mathrm{~min}$. at $25 \mathrm{~V}$ and $300 \mathrm{~mA}$. When finished, slides were neutralized by means of $0.4 \mathrm{M}$ Tris buffer ( $\mathrm{pH} 7.5$, Invitrogen, Blijswijk, the Netherlands), stained with $25 \mu \mathrm{L}$ ethidium bromide (5 $\mu \mathrm{g} / \mathrm{mL}$ in milliQ, CAS no. 1239-45-8, Sigma-Aldrich Zwijndrecht, the Netherlands) and covered with a cover slip. From each slide 100 cells were scored using a fluorescent microscope (Zeiss Axioskop fluorescence microscope, Carl Zeiss BV, Sliedrecht, the Netherlands). Cells were visually divided over score classes ranging from 0 (no tail) to 4 (almost all DNA in the tail) and an average score for 3 slices from each donor, 
compound and dose was determined [28].

\section{Viability of liver slices (cytotoxicity)}

LDH leakage into the culture medium was measured as an indicator of cell viability, using the cytotoxicity detection kit (Roche, Almere, the Netherlands) according to the manufacturers protocol [29]. As a positive control for maximally possible leakage, 3 liver slices from each donor were intentionally lysed through incubation (1 h) in culture medium containing 1\% Triton X-100. Medium from these 3 slices was pooled and LDH leakage was measured in duplicate, as was done for all other samples. Leakage was defined as \% of maximally possible leakage.

\section{RNA isolation}

Slices for RNA isolation were snap-frozen in liquid nitrogen immediately after incubation (in triplicate). Triplicate slices were pooled and $0.5 \mathrm{~mL}$ cold QIAzol (Qiagen, Venlo, the Netherlands) was added to the frozen slices after which the tissue was homogenized using a T10 Ultra-Turrax (IKA, Staufen, Germany). Subsequently total RNA was isolated using the RNeasy mini kit (Qiagen, Venlo, the Netherlands) according to the manufacturer's protocol.

\section{Real Time PCR (RT-PCR)}

For GE analysis by RT-PCR of CYPs (CYP1A1, CYP1A2, CYP1B1, CYP2E1 and CYP3A4), two genes, amongst others, involved in cellular stress response (NQ01, GSTM1), a p53-mediated DNAdamage response gene (CDKN1A (p21)) and a reference gene (B-actin), cDNA was synthesized using the iScript CDNA Synthesis kit (Bio-rad, Veenendaal the Netherlands) according to the manufacturer's protocol, starting with $0.5 \mu \mathrm{g}$ of RNA. For primer sequences see supplementary Table 3, primer efficiency was between 90-110\%. A MyiQ SingleColor Real-Time PCR detection system (Bio-Rad, Veenendaal, the Netherlands) and the iQ SYBR Green Supermix (Bio-Rad, Veenendaal, the Netherlands) were used to perform the PCR according the manufacturer's protocol. The software of the MyiQ system was used to obtain the Ct-values. Delta-Ct $(\Delta \mathrm{Ct})$ values were calculated for all genes versus the reference gene. 


\section{Cytochrome P450 (CYP) enzyme activity}

The CYP1A2, CYP2A6, CYP2B6, CYP2C19, CYP2D6, CYP2C9 and CYP3A4 activities of 3 replicate slices were assessed after 24 hours of culturing using the CYP450 AL cocktail (Advancell, Valencia, Spain) consisting of a CYP substrate mixture which was prepared and used according to Lahoz et al [30]. The mixture contains the following substates: phenacetin (for CYP1A2, CAS no. 62-44-2, Ultrafine, Manchester, United Kingdom), coumarin (for CYP2A6, CAS no. 9164-5, Sigma-Aldrich, Madrid, Spain), bupropion (for CYP2B6, CAS no. 34841-39-9, Sigma-Aldrich, Madrid, Spain), diclofenac (for CYP2D6, CAS no. 15307-86-5, Sigma-Aldrich, Madrid, Spain), mephynytoin (for CYP2C9, CAS no. 50-12-4, Ultrafine, Manchester, United Kingdom) and midazolam (for CYP3A4, CAS no. 59467-70-8, Ultrafine, Manchester, United Kingdom). These genes were chosen because of their involvement in the metabolism of the compounds used within this study [17, 31-33]. After 2 hours of incubation in presence of the mixture, slices were weighted and snap-frozen. Also the culture medium was snap-frozen. In short, medium samples were subjected to hydrolysis of possible conjugates by incubation with B-glucuronidase (CAS no. 900145-0) and arylsulfatase (CAS no. 9016-17-5) for 2 hours at $37^{\circ} \mathrm{C}$ (both purchased from Roche Diagnostics, Mannheim, Germany). Thereafter, one volume of ice-cold acetonitrile was added, centrifuged and supernatants were transferred for further analysis. Metabolites present in the culture medium were quantified by high-performance liquid chromatography/tandem mass spectrometry as previously described [34]. Activities were expressed as picomole of metabolite formed per incubation time per mg tissue.

\section{Benchmark dose (BMD) calculation}

To define at which dose a compound exerts a predefined effect, the Benchmark Dose (BMD) approach was used with the program PROAST (version 21.2) [35]. For each compound and each individual, data-sets were created containing the dose-range data from all donors for each of the response parameters (e.g. cytotoxicity based on LDH leakage, genotoxicity based on COMET class-scores and GE response based on RT-PCR). An automated fit was performed on the data to find the best fitting statistical model. The statistical model that gave the best fit for the majority of the individuals within a data-set was eventually applied to all individuals within this data-set. In order to define a BMD, the critical effect size (CES) was predefined for each of the response parameters. For cytotoxicity (LDH leakage) all data were rescaled such 
that the control was equal to $1 \%$ of total leakage and the CES was defined as $20 \%$ with an upper bound of $100 \%$. For the genotoxicity (COMET) all data were rescaled such that the control equaled a score of 1 instead of 0 and the CES was set at 2 with an upper bound of 5 (instead of 4 due to rescaling). Finally for the GE data (RT-PCR) the CES was defined as a 2-fold difference compared to the control sample.

\section{Spearman's rho correlation analysis and Bayesian network analysis (BNA)}

Spearman's rho correlation analysis and BNA were used to rank absolute output values of the different parameters to a ranking based on individuals. As such, discordances that arise in the comparisons between absolute measurement values of different techniques, caused by technical differences between measurement techniques, could be avoided. To find correlations between the BMDs from the various response parameters, baseline EA levels and baseline GE levels, 2-tailed Spearman correlations analyses were performed using SPSS (version 17.0). When a BMD-value was missing, because the response did not meet the predefined CES criteria, the highest treatment dose that was used for that specific compound was taken as BMD. Furthermore, BNA was applied to the data. This method creates directed acyclic graphs and has been shown to be beneficial in comparison to the classical correlation methods to use in datasets that include noise [36]. This might provide additional information that could not be obtained using the classical methods. This last argument might be favorable in light of the current dataset that includes noise due to the large interindividual variation between donors. BNFinder with input data identical to those used in the Spearman's correlation analyses was used to perform BNA [37]. All baseline GE and AE levels were defined as regulators.

\section{Results}

\section{Baseline CYP enzyme activity and GE levels}

Activities of CYP1A2, CYP2A6, CYP2B6, CYP2C19, CYP2D6, CYP2C9 and CYP3A4 enzymes were measured in non-compoundexposed slices from each individual donor used in the study $(n=10$ donors, pool of 3 slices each). CYP EA varied greatly between donors; activity of CYP3A4 had the lowest variation between donors (3.8-fold) while CYP2C19 activities demonstrated the highest variation (48.8-fold) (see Figure 1A). 
To determine the variation in base-line $\mathrm{GE}$, deltaCt values of expression levels of CYP1A1, CYP1A2, CYP1B1, CYP2E1 and CYP3A4 of unexposed control slices from each individual donor were evaluated (see Figure1B).

Among these 10 donors, there appear to be large interindividual differences in GE levels of individual CYPs ranging from a 9.5-fold variation for CYP2E1 to a >570-fold variation for CYP1A1.
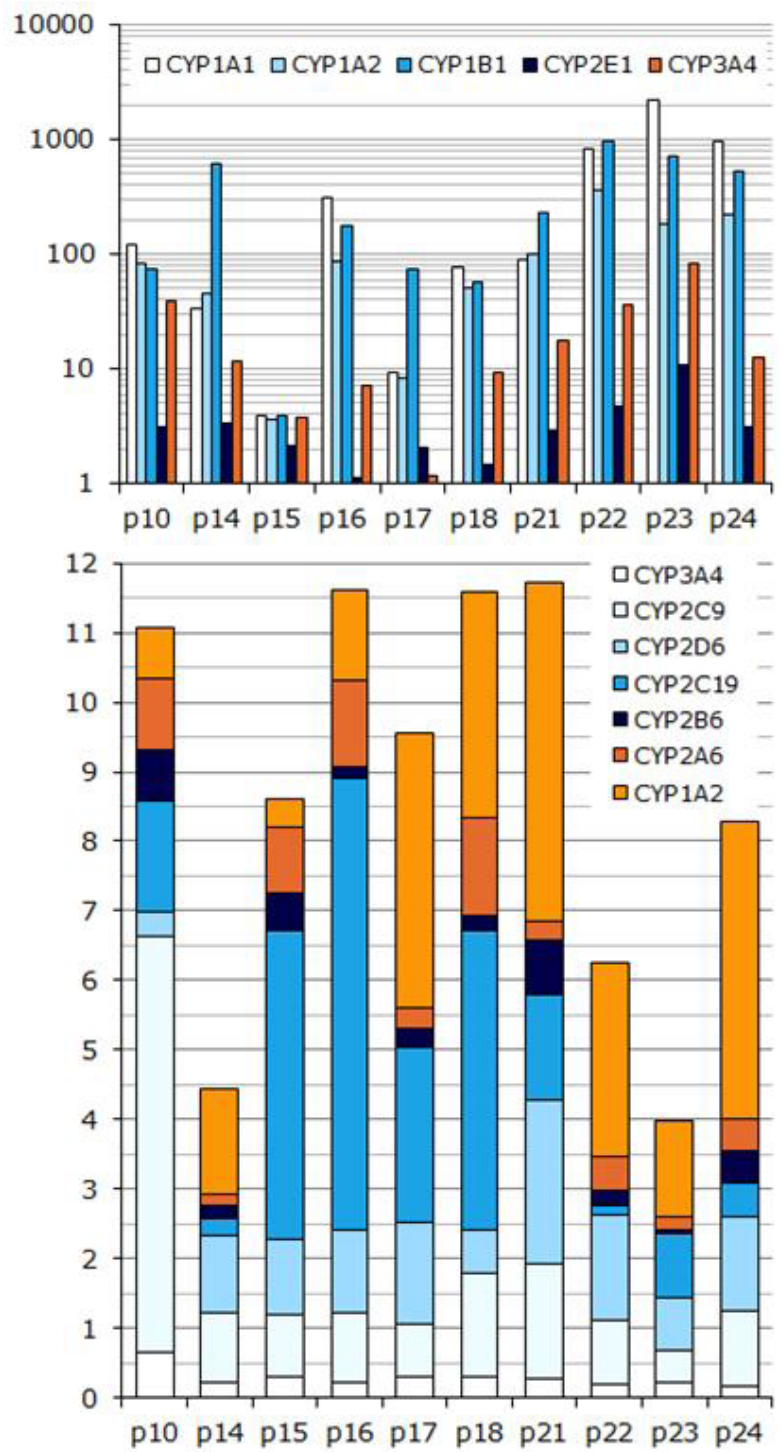

Figure 1; Baseline CYP gene expression levels and CYP enzyme activities

A) Baseline CYP gene expression levels ( $y$-axis; gene expression level $2^{\wedge} \Delta C t$ ) depicted per donor $(x$-axis) and B) cumulative CYP enzyme activities (y-axis; pmol/min/mg tissue) depicted per donor $(x$-axis). 


\section{BMD evaluations for cytotoxicity, DNA-damage and GE}

$\mathrm{BMD}$ values for cytotoxicity (LDH-leakage) are shown in Figure 2A (also see supplementary Table 4). The average BMD for AFB is 4.22 $\mu \mathrm{M}$ with an approximately 2 -fold difference between individuals. APAP has an average BMD of $24.36 \mathrm{mM}$, but with a 400 -fold interindividual variation. $\mathrm{BaP}$, with an average BMD of $62.97 \mu \mathrm{M}$, has an almost 54-fold difference between individuals. Finally, NF has an average BMD of 8.21 $\mu \mathrm{M}$ with a 4-fold interindividual variation.

BMDs for genotoxicity (COMET-assay) are shown in Figure 2B (also see supplementary Table 4). The average BMD for AFB is $4.17 \mu \mathrm{M}$ demonstrating an almost 6-fold difference in BMDs between individuals. APAP has an average BMD of $2.31 \mathrm{mM}$, with a 64-fold interindividual variation. BaP, presenting an average BMD of $19.96 \mu \mathrm{M}$, has a 4.5-fold difference in BMD between individuals. NF has an average BMD of 7.12 $\mu \mathrm{M}$ showing and an almost 2-fold interindividual variation.
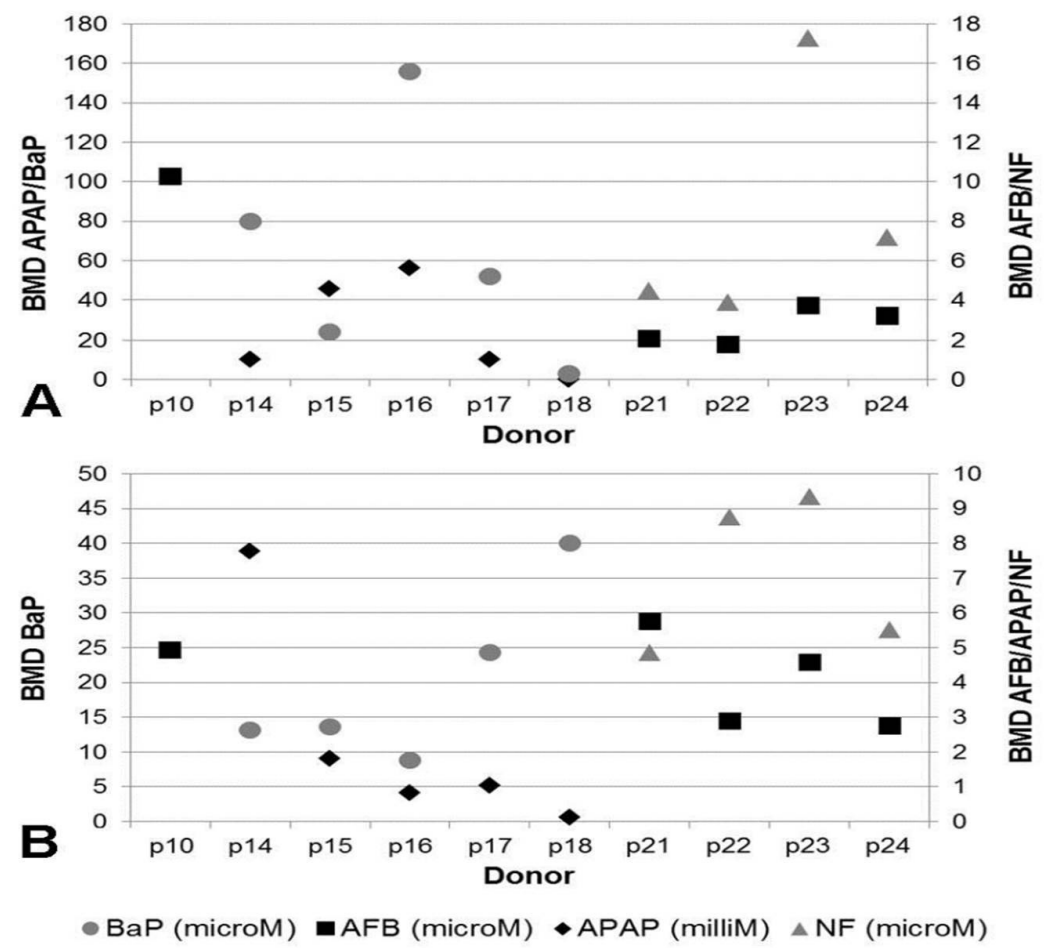

Figure 2; Benchmark doses for cytotoxicity and genotoxicity per individual

Benchmark doses (BMD) calculated for A) $20 \%$ of maximal possible LDH leakage (acetaminophen and benzo(a)pyrene left axis, aflatoxin b1 and 2-nitrofluorene right axis) and B) a COMET scoreclass of 2 after 24 hour compound exposure (benzo( $\alpha$ )pyrene left axis, acetaminophen, aflatoxin b1 and 2-nitrofluorene right axis). 
BMDs for GE changes caused by compound exposure are shown in Figure 3 (also see supplementary Table 4). After AFB exposure, in 3 out of 5 donors for CYP1A2 and all donors for CYP3A4 a BMD could be calculated. In case a BMD could not be calculated due to lack of response within the used dose range, the BMD was set equal to the highest exposure dose $(80 \mu \mathrm{M})$. This resulted in average BMDs of 36.35 $\mu \mathrm{M}$ for CYP1A2 expression and $2.83 \mu \mathrm{M}$ for CYP3A4 expression. An almost 885-fold difference for CYP1A2 and a 2.9-fold difference for CYP3A4 appear to exist between individuals (see Figure 3 AFB). For GSTM1 (average BMD=64.9 $\mu \mathrm{M}$ ) 4 individuals did not show a response that enabled calculation of BMDs, which was therefore set at $80 \mu \mathrm{M}$. The one donor that showed a clear response, resulted in a much lower BMD for this donor and an overall 17-fold interindividual variation. For NQO1 (average BMD=12.95 $\mu \mathrm{M}$ ), a BMD could be calculated for all 5 donors, resulting in a 9-fold interindividual variance in GE levels. Finally, all donors responded for CDKN1A (average BMD=1.97 $\mu \mathrm{M}$ ), with differences in GE expression as large as 44-fold between individuals.

APAP provoked a GE response enabling BMD calculation for 4 out of 5 donors with respect to induced expression levels of CYP1A2, CYP2E1 and CYP3A4, see Figure 3 APAP (also see supplementary Table 4). CYP1A2 has an average BMD of $5.02 \mathrm{mM}$ with a 7-fold difference between donors. For CYP2E1 the average BMD is $3.69 \mathrm{mM}$ with an almost 227-fold variability between individuals. CYP3A4 has an average BMD of $3.04 \mathrm{mM}$ with a 129-fold interindividual variation. An 8.2-fold interindividual variability with an average BMD of $2.4 \mathrm{mM}$ was determined for GSTM1 (BMDs could be calculated for all donors). Also, BMDs could be calculated for the response from CDKN1A for each donor, resulting in an average BMD of $1.97 \mathrm{mM}$ with a 114-fold difference between donors.

BaP exposure resulted in average BMDs of $0.67,3.54$ and 2.25 $\mu \mathrm{M}$ for expression levels of CYP1A1, CYP1A2 and CYP3A4 respectively (BMDs could be calculated for each donor, also see Figure $3 \mathrm{BaP}$ and supplementary Table 4). The interindividual differences in BMDs are almost 12-fold for CYP1A1, 97-fold for CYP1A2 and over 43-fold for CYP1B1. For NQ01, 3 out of 5 donors gave a gene-response that passed the criteria for BMD calculation, resulting in an average BMD of $39.63 \mu \mathrm{M}$ with a 47-fold difference between individuals. For CDKN1A, all donors passed the criteria for BMD calculation with an average BMD of $1.86 \mu \mathrm{M}$ and an over 58-fold interindividual variation. 
After exposure to NF, a 10.4-fold difference between donors is seen in BMDs for CYP1A1 expression with an average BMD of 2.74 $\mu \mathrm{M}$ (BMD calculation possible for each donor, see Figure $3 \mathrm{NF}$ and supplementary Table 4). For CYP1A2, for all but one donor a BMD could be determined, giving an average BMD of $21.16 \mu \mathrm{M}$ with an almost 140-fold difference between individuals. Also CYP1B1 showed a response passing BMD calculation criteria for each donor, resulting in an average BMD of $4.71 \mu \mathrm{M}$ with an almost 64-fold interindividual variation. Although 2 donors did not show a response passing BMD calculation criteria for NQO1 $(B M D=80 \mu \mathrm{M})$, the average BMD was $50.42 \mu \mathrm{M}$ with a 7.4-fold interindividual variability. CDKN1A changes passed BMD calculation criteria for all donors with an average BMD of $20.03 \mu \mathrm{M}$ and an almost 20-fold difference between donors.
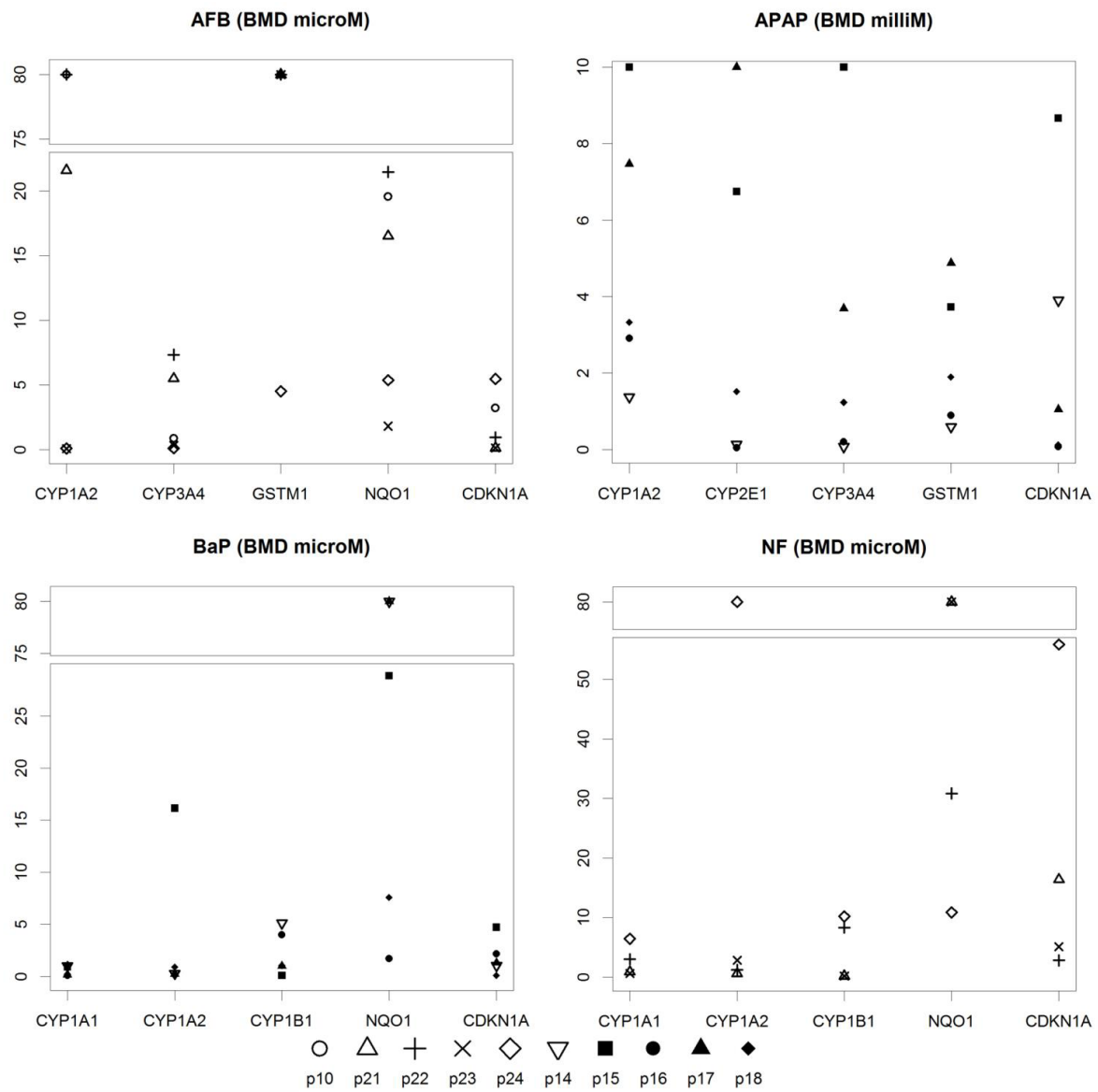

Figure 3; Gene expression responses to compound exposure

Gene expression changes depicted as BMD (y-axis) per gene (x-axis) and donor after 24 hour compound exposure $(A F B=$ Aflatoxin $B 1, A P A P=$ Acetaminophen, $B a P=B e n z O(\alpha)$ pyrene and NF $=$ 2-Nitrofourene). 


\section{Spearman's rho correlation and BNA}

Spearmen's correlations and Bayesian connections between baseline CYP GE levels/EA levels and at least one of the response parameters could be found for all compounds (Figure 4). In addition, correlations between the different response parameters were also found for all compounds except NF. Several Bayesian connections corresponded with the correlations from the Spearman analyses. There is a difference in the number of correlations/connections found for the various compounds, with $A P A P>A F B>B a P>N F$. In addition, more Bayesian connections were found than Spearman's correlations. For all compounds, the parameters that are most often detected by either of the above described statistical approaches are the genotoxicity, cytotoxicity and gene-expression responses of genes involved in DNAdamage and/or cellular stress responses. Concerning interindividual variation, classic parameters for genotoxicity (COMET/LDH) show lower levels of variation in comparison to EA/GE related parameters. However, especially BNA demonstrates that the latter parameters influence the outcome of genotoxicity and cytotoxicity in a donor specific manner.
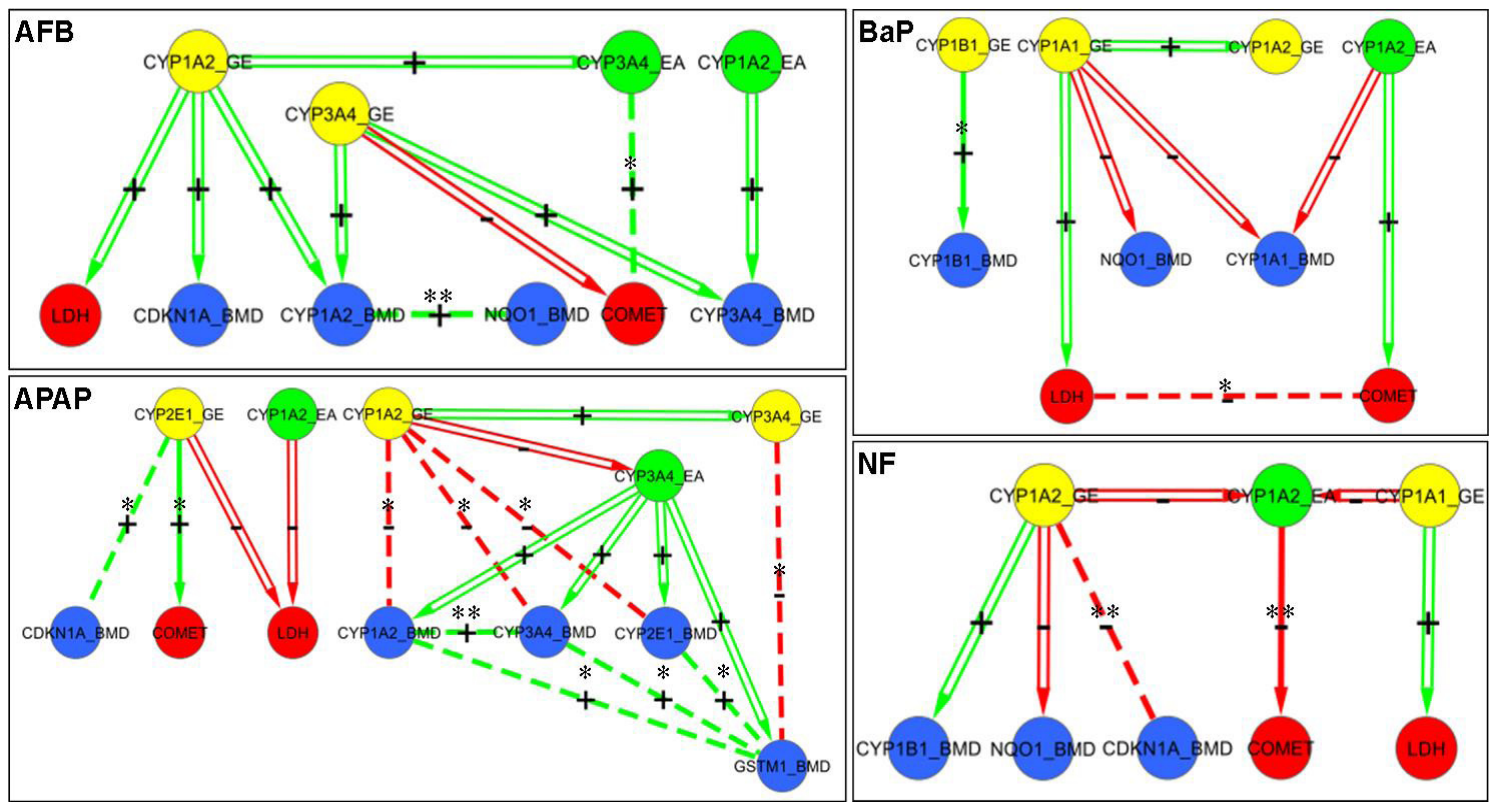

Figure 4; Compound related networks

A network was created for each compound consisting of both Bayesian connections and Spearman's correlations. Parallel line arrows; Bayesian connection, dotted line; Spearman's correlation, solid arrow; connected by Bayesian and Spearman's correlation. For Spearman's correlation ${ }^{*}=p<0.05$ and ${ }^{*}=p<0.01$. GE $=$ baseline gene expression (yellow), EA = baseline enzyme activity (green), BMD = benchmark dose (blue), COMET/LDH (red), positive connections in green, negative connections in red. 


\section{Discussion}

In this study we aimed at evaluating interindividual variation in precision-cut human liver slices. Additionally, we hypothesized that modelling response networks, using interindividual variation in baseline and response parameters, defines key parameters causing variability in toxicity responses.

AFB exerts cytotoxic and genotoxic effects on liver cells [3]. In our study, the interindividual variability in AFB-derived BMDs for cytotoxicity and genotoxicity varied $<6$-fold, while those of response genes varied $>10$-fold (Figure $2 /$ supplementary Table 4 ). AFB-induced DNA-adduct levels have been reported to vary 51-fold in AFB-exposed liver microsomal preparations, with a 131-fold interindividual variation in AFB-protein adducts which are responsible for AFB's cytotoxic effects [17]. Also, a >20-fold difference in DNA-damage levels has been described in AFB-exposed hepatocytes [38]. CYP1A2/3A4 are key-players in the metabolism of $A F B$, involved in both the formation of toxic AFB-metabolites and non-toxic AFB-clearance [3]. Both enzymes are linked with the LDH and COMET parameters through BNA, suggesting a role in causing variance in AFB-metabolism (Figure 4, AFB). CYP1A2 is involved in the formation of the toxic AFB-metabolite, since donors with the lowest levels of baseline CYP1A2 GE are least affected by cytotoxicity. Also, CDKN1A is connected to baseline CYP1A2 GEresponses. CDKN1A acts in the DNA-damage response and is known to vary between individuals in baseline liver mRNA expression-levels (11-fold in normal tissue, 40-fold in tumours [39, 40]). Lower baseline CYP1A2 GE-levels correspond to a CDKN1A GE-response at lower BMDs, possibly preventing DNA-damage from occurring. Individuals with higher baseline CYP3A4 GE-levels suffer DNA-damage at lower BMDs, suggesting formation of DNA-damaging AFB-metabolites by CYP3A4. However, low baseline CYP3A4 EA-levels correlating to low BMDs for DNA-damage suggest an opposite role for CYP3A4, indicating a dual role for this enzyme in AFB-metabolism. A low BMD for CYP1A2 GE-response corresponds to a low BMD for NQO1 GE-response, which is involved in the oxidative stress response and possibly reduces cellular stress and cytotoxicity [41]. Summing up, the network created in this study indicates a dual role for CYP1A2/3A4 in AFB-metabolism in line with literature $[3,17,42]$. In addition, this study shows that interindividual baseline variation in CYP1A2 causes variability in both cytotoxicity and genotoxicity outcome parameters, while variation in baseline CYP3A4 causes variability that is more genotoxicity-oriented. This demonstrates that network modelling is helpful in defining crucial 
parameters based on interindividual variation.

Butterworth et al. reported an $>20$-fold difference in DNAdamage levels in BaP-exposed hepatocytes from different individuals [38]. The fold-difference in BMDs calculated from the COMET-assay after $\mathrm{BaP}$ exposure is $<10$-fold, while variation in BMDs calculated from LDH-leakage is $>50$-fold (Figure 2/supplementary Table 4). In general, the BMDs for genotoxicity are lower than the BMDs for cytotoxicity i.e. genotoxicity occurs at lower doses than cytotoxicity. The negative correlation between the responses of LDH-leakage/COMET-assay in the network for BaP corroborates this (Figure 4). CYP1A1 GE and CYP1A2 EA are linked with the LDH and COMET parameters respectively, demonstrating their role in variability in $\mathrm{BaP}$-metabolism. $\mathrm{BaP}$ is primarily metabolized through CYP1A1/1A2/1B1 $[3,43]$. These enzymes are involved in both the formation of genotoxic BaP-metabolites, like $B P D E$, and the non-toxic metabolism of BaP. Those individuals with the lowest baseline CYP1A1 GE-levels (and indirectly CYP1A2) have a low BMD for LDH-leakage, indicating an increased BaP-toxicity sensitivity for individuals having low baseline CYP1A1 GE. Also, individuals with low endogenous CYP1A1 GE-levels respond at higher BMDs with respect to NQO1 GE, meaning that individuals with a low endogenous CYP1A1 GE-level seem to have less oxidative stress thus indicating a toxifying role for CYP1A1. In addition, a low baseline CYP1A2 EAlevel in an individual corresponds with a low BMD for DNA-damage. This indicates an involvement of CYP1A2 in the non-BPDE forming branch of the metabolism of BaP. Therefore, individuals with lower CYP1A2 EA-levels have less non-BPDE related metabolism of BaP and as a result have more BaP available for the formation of BPDE, as such increasing the susceptibility to DNA-damage [3, 44]. Lastly, several connections between the CYP parameters are depicted in the BaPrelated network. BaP is capable of inducing the levels of CYPs through the Aryl hydrocarbon Receptors (AhR) [45]. CYP1A1/1A2/1B1 are under regulation of this receptor, which explains the links between the different CYPs in the network. Summarized, network analysis indicates baseline CYP expression levels are important sources for interindividual variation in toxicity measures. This study defines CYP1A1/1A2 as factors of larger influence on variability in cytotoxicity/genotoxicity than CYP1B1.

This study shows that NF induces cytotoxicity with a 4.5fold difference between individuals and genotoxicity with an almost 2-fold difference between individuals (Figure 2/supplementary Table 4). BMD data derived from the LDH-test and COMET-assay are placed 
in the NF-related network (Figure 4). NF is mainly bio-activated by CYP1A2, resulting in several toxic metabolites. In addition to CYP1A2, a metabolizing role for CYP1A1 has been suggested [46-49]. BMDs from the COMET-assay are negatively correlated to baseline CYP1A2 EA-levels, meaning that a low level of endogenous CYP1A2 EA in an individual, and therefore a slower formation of genotoxic metabolites, leads to a lower level of DNA-damage. A similar connection exists between baseline CYP1A2 GE-levels and the NQ01/CDKN1A GEresponse. Both connections indicate that individuals with low CYP1A2 GE-levels, and thus less toxic NF-metabolite formation, react at higher BMDs with an oxidative stress/DNA-damage related response, confirming the role of CYP1A2 as NF-activating enzyme. Also CYP1A1 has been proposed as NF-metabolizing CYP, and is indeed placed within the NF-network. Since low baseline CYP1A1 GE-levels in an individual are linked to a high LDH-BMD, a toxifying role for CYP1A1 in NFmetabolism is suggested. As in the BaP-related network, CYP-associated parameters link to each other; this probably is a consequence of their shared regulatory mechanism through AhR. In short, CYP1A1/1A2 are both defined as sources for interindividual variation in toxicity responses in network analysis. In addition, this study suggests a NF-metabolizing role for CYP1B1. Since CYP1B1 is also under regulation of the AhR jointly with CYP1A1/1A2 its involvement in NF-metabolism is plausible, however we are not aware of any literature defining CYP1B1 as NFmetabolizer.

Although APAP is a non-carcinogen, both APAP and its oxidative metabolite NAPQI are known to covalently bind DNA in mouse-liver microsomal incubations and cause DNA-damage [50, 51]. In addition, the formation of reactive oxygen species during metabolism of APAP is an additional mechanism in which DNA-damage can be induced [52]. To our knowledge there is no human data available on possible interindividual variation in the level of DNA-damage caused by APAP, or the interindividual variation in the DNA-damage response triggered by APAP in in vitro models. However, our study does show a large variability in BMDs for genotoxicity (>60-fold) and cytotoxicity (400fold, Figure 2/supplementary Table 4). This variation is well above the variation seen with the other substances, which fits with the high variability in toxicity responses to APAP that is observed within the human population [53]. High baseline CYP1A2 EA-levels correspond with a low LDH-BMD, suggesting that individuals with high CYP1A2 EA-levels generate more NAPQI and therefore suffer cytotoxicity at lower doses (Figure 4). An identical link is found between baseline CYP2E1 GE-levels and the LDH-BMD, suggesting that also this CYP is 
involved in the formation of NAPQI. In addition, an individual with a low baseline CYP1A2 GE-level has a low BMD for genotoxicity, meaning that lower levels of APAP-conversion cause increased DNA-damage levels, which fits with APAPs DNA-binding capacity. The correlation between baseline CYP2E1 GE-levels and the BMD for CDKN1A again suggests the same. GSTM1 is involved in the phase-Il metabolism of APAP where it serves as a catalyst for NAPQI-conjugation to glutathione in the liver [54]. Depletion of glutathione, through a NAPQI-overload, results in conjugation of NAPQI to vital cell elements, leading to liver damage [55]. While GSTM1 is known to be highly polymorphic within the human population [56], genetic variants in GSTM1 so far could not be linked to toxicity in APAP-poisoned patients [57]. However, the present study suggests that GSTM1 causes variability in APAP-metabolism that is dependent on the GE-responses of CYP enzymes within an individual. In a rodent based study, GSTM1 has been proposed as a mediating factor in cellular signaling after APAP-induced hepatotoxicity, by influencing the phosphorylation of c-jun N-terminal kinase, which is involved in the necrosis related response in hepatocytes after APAP-induced toxicity [58]. To our knowledge, this finding has not yet been confirmed in humans. Taken together, the network-analysis suggests baseline CYP GE-levels as factors for variability in cytotoxicity/genotoxicity responses towards APAP exposure. Especially, CYP1A2 seems to be a key parameter causing interindividual variation, also influencing GSTM1.

In general, there is only a limited number of connections between baseline GE levels and baseline AE levels (see Figure 3), which as such is not entirely unexpected. It has been shown before that only about only $54-77 \%$ of the variance in baseline average protein levels in E.Coli can be explained by the baseline mRNA concentrations [59]. During the response of a cell to a stimulus, correlations between protein and mRNA levels again change [60]. To obtain a more complete view of the dynamics involved in the balance between gene transcript levels and protein levels, the inclusion of factors like miRNAs, RNA-binding proteins and gene methylation-status could provide additional information in future analysis.

In conclusion, interindividual variation in GE/EA-levels in most cases exceeds the interindividual variation in phenotypical endpoint responses evaluated here (LDH/COMET), making the latter more robust assays for measuring functional toxicity responses. In cases where knowledge on interindividual variation in toxicity-response is of interest, network analyses provide insight in the factors influencing such variation, in this study, by defining the metabolizing CYPs as 
key parameters. This method also allows for the discovery of new or unexpected factors, like the involvement of CYP1B1 in NF-metabolism and the link with GSTM1 in the APAP-network. Therefore we believe that network modelling for compound specific dose-response relationships, based on interindividual variation, might prove to be a helpful tool in future endeavors in which handling of interindividual variation is essential, for instance in human risk-assessment.

\section{Supplementary Tables / Figures}

Supplementary Table 1-4 and Supplementary Figure 1 Available online: http://www.sciencedirect.com/science/article/pii/ S0300483X14001206

\section{Acknowledgements}

This work was supported by CarcinoGENOMICS, a part of the 6th framework project of the European Union aimed at developing in vitro methods for assessing the carcinogenic potential of compounds, as an alternative to current rodent bioassays for genotoxicity and carcinogenicity. Furthermore, the authors would like to thank Prof. dr. G.M.M. Groothuis and her colleagues at the Department of Drug Metabolism and Toxicology of the Rijksuniversiteit Groningen, The Netherlands for their help with the set-up of the precision cut liver slice technique and the development of the protocol for the COMETassay on liver slices. Also the authors would like to thank Dr. D. Jennen (Department of Toxicogenomics, Maastricht University, The Netherlands) for his assistance with the Bayesian network analysis and D. Theunissen (Department of Toxicogenomics, Maastricht University, The Netherlands) for his help with the graphics. 


\section{References}

1. Hardisty, J.F. and A.E. Brix, Comparative hepatic toxicity: prechronic/chronic liver toxicity in rodents. Toxicol Pathol, 2005. 33(1): p. 35-40.

2. Guengerich, F.P., Cytochrome p450 and chemical toxicology. Chem Res Toxicol, 2008. 21(1): p. 70-83.

3. Zhou, S.F., et al., Structure, function, regulation and polymorphism and the clinical significance of human cytochrome P450 1A2. Drug Metab Rev, 2010. 42(2): p. 268-354.

4. Chang, T.K., et al., Real-time polymerase chain reaction analysis of CYP1B1 gene expression in human liver. Toxicol Sci, 2003. 71(1): p. 11-9.

5. Rodriguez-Antona, C., et al., Cytochrome P-450 mRNA expression in human liver and its relationship with enzyme activity. Arch Biochem Biophys, 2001. 393(2): p. 308-15.

6. Schweikl, H., et al., Expression of CYP1A1 and CYP1A2 genes in human liver. Pharmacogenetics, 1993. 3(5): p. 239-49.

7. Wang, D., et al., Functional evaluation of genetic and environmental regulators of p450 mRNA levels. PLoS One, 2011. 6(10): p. e24900.

8. Rhodes, S.P., et al., Simultaneous assessment of cytochrome P450 activity in cultured human hepatocytes for compound-mediated induction of CYP3A4, CYP2B6, and CYP1A2. J Pharmacol Toxicol Methods, 2011. 63(3): p. 223-6.

9. Hakura, A., et al., Use of human liver $\$ 9$ in the Ames test: assay of three pro carcinogens using human $\$ 9$ derived from multiple donors. Regulatory Toxicology and Pharmacology, 2003. 37(1): p. 20-27.

10. Kamdem, L.K., et al., Dominant contribution of P450 3A4 to the hepatic carcinogenic activation of aflatoxin B1. Chem Res Toxicol, 2006. 19(4): p. 577-86.

11. Shimada, T., et al., Interindividual variations in human liver cytochrome P-450 enzymes involved in the oxidation of drugs, carcinogens and toxic chemicals: studies with liver microsomes of 30 Japanese and 30 Caucasians. J Pharmacol Exp Ther, 1994. 270(1): p. 414-23.

12. Thummel, K.E., et al., Oxidation of acetaminophen to N-acetyl-paminobenzoquinone imine by human CYP3A4. Biochem Pharmacol, 1993. 45(8): p. 1563-9.

13. Powell, H., et al., Expression of cytochrome P4502E1 in human liver: assessment by mRNA, genotype and phenotype. Pharmacogenetics, 1998. 8(5): p. 411-21.

14. Westlind, A., et al., Cloning and tissue distribution of a novel human cytochrome p450 of the CYP3A subfamily, CYP3A43. Biochem Biophys Res Commun, 2001. 281(5): p. 1349-55.

15. Lamba, V., et al., Genetic predictors of interindividual variability in hepatic CYP3A4 expression. J Pharmacol Exp Ther, 2010. 332(3): p. 1088-99.

16. Raucy, J.L., Regulation of CYP3A4 expression in human hepatocytes by pharmaceuticals and natural products. Drug Metab Dispos, 2003. 31(5): p. 533-9.

17. Doi, A.M., P.E. Patterson, and E.P. Gallagher, Variability in aflatoxin B(1)macromolecular binding and relationship to biotransformation enzyme expression in human prenatal and adult liver. Toxicol Appl Pharmacol, 2002. 181(1): p. 48-59.

18. Cascorbi, I., Genetic basis of toxic reactions to drugs and chemicals. Toxicol Lett, 2006. 162(1): p. 16-28.

19. de Graaf, I.A., et al., Preparation and incubation of precision-cut liver and 
intestinal slices for application in drug metabolism and toxicity studies. Nat Protoc, 2010. 5(9): p. 1540-51.

20. Lee, W.M., Acetaminophen toxicity: changing perceptions on a social/medical issue. Hepatology, 2007. 46(4): p. 966-70.

21. Hernandez, L.G., et al., Can carcinogenic potency be predicted from in vivo genotoxicity data?: a meta-analysis of historical data. Environ Mol Mutagen, 2011. 52(7): p. 518-28.

22. Dejong, C.H.C. and O.J. Garden, Neoplasms of the liver. In: Advanced Surgical Practice, edited by Majid AA and Kingsnorth A London: Greenwich Medical Media, 2003: p. 146-156.

23. Jennen, D.G., et al., Comparison of HepG2 and HepaRG by whole-genome gene expression analysis for the purpose of chemical hazard identification. Toxicological sciences : an official journal of the Society of Toxicology, 2010. 115(1): p. 66-79.

24. Jetten, M.J., et al., Baseline and genotoxic compound induced gene expression profiles in HepG2 and HepaRG compared to primary human hepatocytes. Toxicology in vitro : an international journal published in association with BIBRA, 2013. 27(7): p. 2031-40.

25. Mathijs, K., et al., Discrimination for genotoxic and nongenotoxic carcinogens by gene expression profiling in primary mouse hepatocytes improves with exposure time. Toxicological sciences : an official journal of the Society of Toxicology, 2009. 112(2): p. 374-84.

26. Plazar, J., et al., Detection of xenobiotic-induced DNA damage by the comet assay applied to human and rat precision-cut liver slices. Toxicol In Vitro, 2007. 21(6): p. 1134-42.

27. Langie, S.A., et al., Development and validation of a modified comet assay to phenotypically assess nucleotide excision repair. Mutagenesis, 2006. 21(2): p. 153-8.

28. da Silva, J., et al., An alkaline single-cell gel electrophoresis (COMET) assay for environmental biomonitoring with native rodents. Genet. Mol. Biol., 2000. 23(1): p. 241-245.

29. Morand, S., et al., Induction of apoptosis in human corneal and HeLa cells by mutated BIGH3. Invest Ophthalmol Vis Sci, 2003. 44(7): p. 2973-9.

30. Lahoz, A., et al., Strategies to in vitro assessment of major human CYP enzyme activities by using liquid chromatography tandem mass spectrometry. Curr Drug Metab, 2008. 9(1): p. 12-9.

31. Dong, H., et al., Knock-in mouse lines expressing either mitochondrial or microsomal CYP1A1: differing responses to dietary benzo[a]pyrene as proof of principle. Mol Pharmacol, 2009. 75(3): p. 555-67.

32. Fujimoto, T., et al., Estrogenic activity of an environmental pollutant, 2-nitrofluorene, after metabolic activation by rat liver microsomes. Biochem Biophys Res Commun, 2003. 303(2): p. 419-26.

33. Hazai, E., L. Vereczkey, and K. Monostory, Reduction of toxic metabolite formation of acetaminophen. Biochem Biophys Res Commun, 2002. 291(4): p. 1089-94.

34. Lahoz, A., et al., Determination of major human cytochrome P450s activities in 96-well plates using liquid chromatography tandem mass spectrometry. Toxicol In Vitro, 2007. 21(7): p. 1247-52.

35. Slob, W., et al., A statistical evaluation of toxicity study designs for the estimation of the benchmark dose in continuous endpoints. Toxicol Sci, 2005. 84(1): p. 167-85.

36. Behseta, S., et al., Bayesian correction for attenuation of correlation in multi- 
Wilczynski, B. and N. Dojer, BNFinder: exact and efficient method for learning Bayesian networks. Bioinformatics, 2009. 25(2): p. 286-7.

38. Butterworth, B.E., et al., Use of primary cultures of human hepatocytes in toxicology studies. Cancer research, 1989. 49(5): p. 1075-84.

39. Qin, L.F. and I.O. Ng, Expression of p27(KIP1) and p21(WAF1/CIP1) in primary hepatocellular carcinoma: clinicopathologic correlation and survival analysis. Hum Pathol, 2001. 32(8): p. 778-84.

40. Cazzalini, O., et al., Multiple roles of the cell cycle inhibitor p21(CDKN1A) in the DNA damage response. Mutat Res, 2010. 704(1-3): p. 12-20.

41. Dinkova-Kostova, A.T. and P. Talalay, NAD(P)H:quinone acceptor oxidoreductase 1 (NQO1), a multifunctional antioxidant enzyme and exceptionally versatile cytoprotector. Arch Biochem Biophys, 2010. 501(1): p. 116-23.

42. Eaton, D.L. and E.P. Gallagher, Mechanisms of aflatoxin carcinogenesis. Annual review of pharmacology and toxicology, 1994. 34: p. 135-72.

43. Ma, Q. and A.Y. Lu, CYP1A induction and human risk assessment: an evolving tale of in vitro and in vivo studies. Drug Metab Dispos, 2007. 35(7): p. 1009-16.

44. Bauer, E., et al., Oxidation of benzo[a]pyrene by recombinant human cytochrome P450 enzymes. Chemical research in toxicology, 1995. 8(1): p. $136-42$.

45. Endo, K., et al., Inhibition of aryl hydrocarbon receptor transactivation and DNA adduct formation by CYP1 isoform-selective metabolic deactivation of benzo[a]pyrene. Toxicol Appl Pharmacol, 2008. 230(2): p. 135-43.

46. Moller, L., In vivo metabolism and genotoxic effects of nitrated polycyclic aromatic hydrocarbons. Environ Health Perspect, 1994. 102 Suppl 4: p. 139-46.

47. Kappers, W.A., et al., Comparison of three different in vitro mutation assays used for the investigation of cytochrome P450-mediated mutagenicity of nitropolycyclic aromatic hydrocarbons. Mutat Res, 2000. 466(2): p. 143-59.

48. Ellinger-Ziegelbauer, $\mathrm{H}_{\text {., }}$ et al., Characteristic expression profiles induced by genotoxic carcinogens in rat liver. Toxicol Sci, 2004. 77(1): p. 19-34.

49. Lecureur, V., A. Guillouzo, and O. Fardel, Differential regulation of mdr genes in response to 2-acetylaminofluorene treatment in cultured rat and human hepatocytes. Carcinogenesis, 1996. 17(5): p. 1157-60.

50. Dybing, E., et al., Genotoxicity studies with paracetamol. Mutat Res, 1984. 138(1): p. 21-32.

51. Rannug, U., et al., International Commission for Protection against Environmental Mutagens and Carcinogens. An evaluation of the genetic toxicity of paracetamol. Mutat Res, 1995. 327(1-2): p. 179-200.

52. Jaeschke, H., M.R. McGill, and A. Ramachandran, Oxidant stress, mitochondria, and cell death mechanisms in drug-induced liver injury: lessons learned from acetaminophen hepatotoxicity. Drug metabolism reviews, 2012. 44(1): p. 88-106.

53. Spielberg, S.P., Acetaminophen toxicity in human lymphocytes in vitro. The Journal of pharmacology and experimental therapeutics, 1980. 213(2): p. 395-8.

54. Zhao, L. and G. Pickering, Paracetamol metabolism and related genetic differences. Drug Metab Rev, 2011. 43(1): p. 41-52.

55. Mitchell, J.R., et al., Acetaminophen-induced hepatic injury: protective role of glutathione in man and rationale for therapy. Clin Pharmacol Ther, 1974. 16(4): p. 676-84. 
56. Gattas, G.J. and J.A. Soares-Vieira, Cytochrome P450-2E1 and glutathione S-transferase mu polymorphisms among Caucasians and mulattoes from Brazil. Occup Med (Lond), 2000. 50(7): p. 508-11.

57. Buchard, A., et al., The role of the glutathione S-transferase genes GSTT1, GSTM1, and GSTP1 in acetaminophen-poisoned patients. Clin Toxicol (Phila), 2012. 50(1): p. 27-33.

58. Arakawa, S., et al., Resistance to acetaminophen-induced hepatotoxicity in glutathione S-transferase Mu 1-null mice. The Journal of toxicological sciences, 2012. 37(3): p. 595-605.

59. Taniguchi, Y., et al., Quantifying E. coli proteome and transcriptome with single-molecule sensitivity in single cells. Science, 2010. 329(5991): p. 533-8.

60. Vogel, C. and E.M. Marcotte, Insights into the regulation of protein abundance from proteomic and transcriptomic analyses. Nature reviews. Genetics, 2012. 13(4): p. 227-32. 


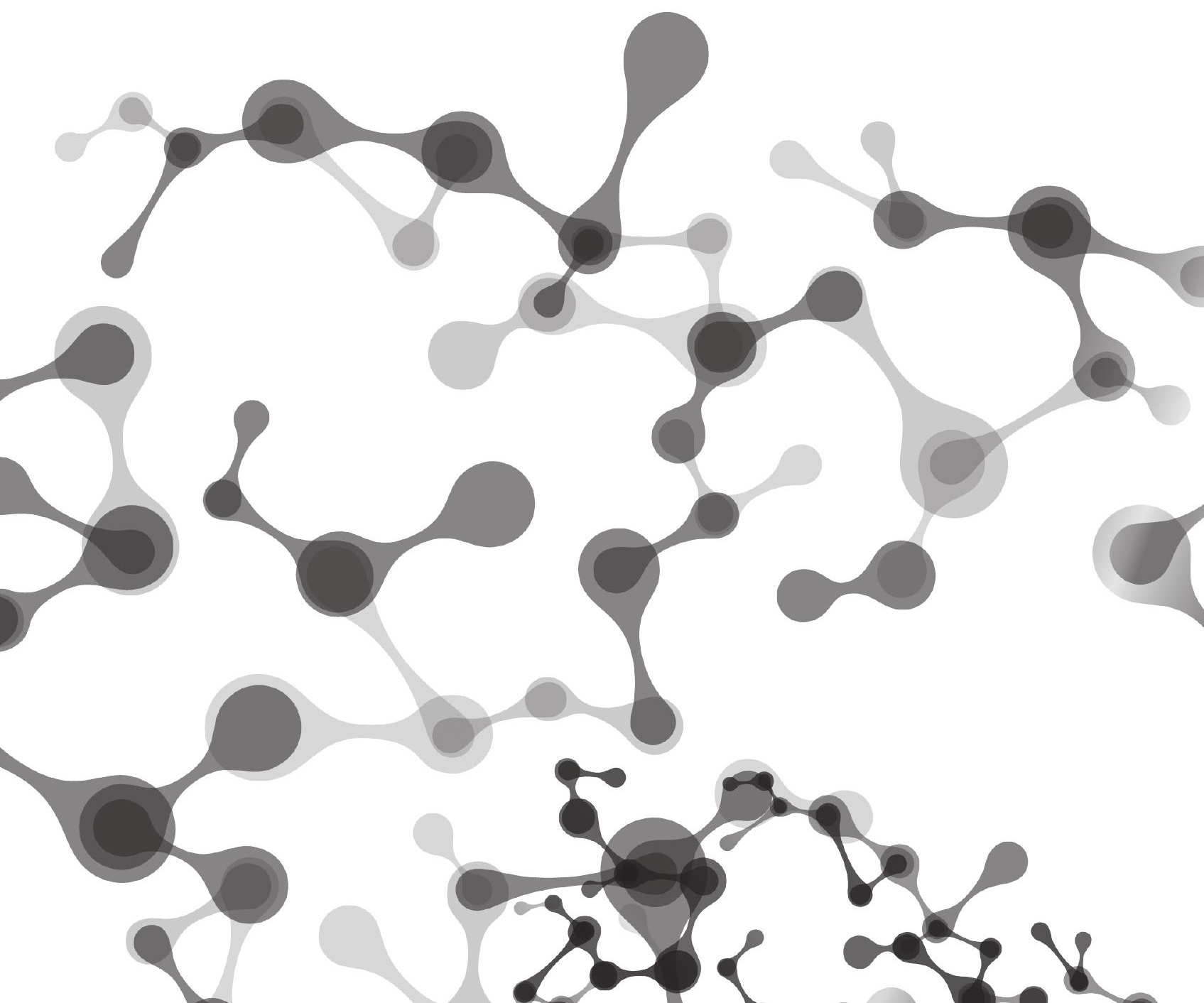




\section{Chapter 3}

Interindividual variation in gene expression responses and metabolite formation in

Acetaminophen-exposed primary human hepatocytes

Marlon J.A. Jetten

Ainhoa Ruiz-Aracama

Maarten L.J. Coonen

Sandra M. Claessen

Marcel H.M. van Herwijnen

Arjen Lommen

Joost H.M. van Delft

Ad A.C.M. Peijnenburg Jos C.S. Kleinjans 


\section{Abstract}

Acetaminophen (APAP) is a readily available over-the-counter drug and is one of the most commonly used analgesics/antipyretics world-wide. Large interindividual variation in susceptibility towards APAP-induced liver failure has been reported. However, the exact underlying factors causing this variability in susceptibility are still largely unknown.

The aim of this study is to better understand this variability in response to APAP by evaluating interindividual differences in gene expression changes and APAP-metabolite formation in primary human hepatocytes from several donors $(n=5)$ exposed in vitro to a non-toxic to toxic APAP dose-range. Those genes demonstrating the highest interindividual variability in their expression levels were used to investigate the inter-individual variability in APAP-metabolite levels. Gene set over-representation analysis was applied for enabling biological interpretations of the transcriptomic results.

The biological processes in which the genes with high interindividual variation in expression are involved include liver regeneration, inflammatory responses, mitochondrial stress responses, hepatocarcinogenesis, cell cycle and drug-efficacy. Additionally, the interindividual variation in the expression of these genes could be associated with the variability in expression levels of hydroxyl/methoxyAPAP and C8H13O5N-APAP-glucuronide. The before mentioned metabolites or their derivatives have also been reported in blood of human individuals exposed to therapeutic APAP-doses.

Possibly these findings can contribute to elucidating the causative factors of interindividual susceptibility towards APAP, as such thriving the research behind the unknown mechanisms behind druginduced liver failure. 


\section{Introduction}

Acetaminophen (APAP) is one of the most commonly used analgesics/antipyretics world-wide. Thereby, it is a readily available overthe-counter drug. Apart from its beneficial effects as a pharmaceutical, APAP is also the most common cause of acute liver toxicity in Europe and the USA [1]. Cytochrome P450 enzymes in the liver metabolize APAP to the oxidative metabolite $\mathrm{N}$-acetyl-p-benzoquinone imine (NAPQI), which is thought to cause the toxic effects of APAP through protein-adduct formation leading to oxidative stress and finally liver damage [2]. The molecular mechanism behind further progression of APAP toxicity still is not fully elucidated; the involvement of multiple mechanisms of toxicity, like inflammatory responses and oxidative stress, has been suggested [3].

The main body of our knowledge on the toxicological, or more specific hepatotoxic, mechanisms of compounds, including APAP, is based on data collected from studies using animal models [4]. However, increasing criticism on the usability and applicability of animal data to the human situation has developed over the last years [5]. Therefore, there is a growing need for human in vitro models such as hepatic cell lines, liver slices, or primary hepatocytes cultures, to facilitate humanbased research.

Large interindividual differences in response to xenobiotic exposure between humans have been documented [6]. In the domains of toxicology/pharmacology many attempts are being undertaken to gain a better understanding of the factors that are causative of this interindividual variation. Environmental factors as well as genetic factors have been proposed to contribute to the variation in drug responses between human individuals. Over the last decade, with the rise of the whole genome 'omics techniques, it has become feasible to perform more complete/in-depth analysis of the genomic components contributing to interindividual variation in the human population [7].

Also, the metabolism of APAP is known to show large interindividual variability [6]. Genetic factors including many biotransformation-related genes, such as UDP-glucuronosyltransferases and cytochrome P450 enzymes, have been suggested to be causative of the variation in APAP-induced adverse effects observed between individuals [6, 8-10]. While some individuals seem to be able to endure APAP-doses considerably exceeding the recommended maximal daily dose, others are at risk of liver toxicity due to APAP much closer to 
the recommended dose-window [11] The relatively high frequency of unintentional over-dosing has recently led the FDA to adjusting recommendations on safe APAP-dosage use by lowering the maximal therapeutic daily dose and decreasing the single dose units of APAP [12].

However, interindividual differences do not only occur at supratherapeutic doses, also (sub-) therapeutic doses of APAP have been shown to cause interindividual variation in APAP-metabolite levels, as well as in mRNA and miRNA expression levels [13]. In the same study, regulation of biological processes known to be related to the liver toxicity response after APAP over-dosing, could be detected at lower and supposedly non-toxic doses.

In this study we aim at investigating the interindividual differences in response to a non-toxic to toxic APAP dose-range, using an in vitro cell model consisting of primary human hepatocytes of several donors. The interindividual differences in APAP-metabolite formation and gene expression responses were considered and compared in an attempt to pinpoint the factors that could be causative of interindividual variation in APAP-metabolism.

\section{Materials and Methods}

\section{Cell culture and treatment}

\section{Primary Human Hepatocytes}

Cryopreserved PHH of 5 individuals (see Supplementary Table 1 for donor demographics) were purchased from Life technologies (Gibco). Cells were cultured in 12-well plates in a collagen sandwich, according to the supplier's protocol [14]. The following culture media were used: Cryopreserved Hepatocyte Recovery Medium (CHRM, Gibco) for thawing, William's medium E (WME)+Glutamax (Gibco) substituted with 10\% FCS (Gibco), 0.02\% penicillin/streptomycin (Gibco) and 0,1 $\mathrm{U} / \mathrm{ml}$ insulin (Invitrogen) for seeding/attaching and WME+Glutamax substituted with $0.02 \%$ Pen/Strep, $0.1 \mathrm{U} / \mathrm{ml}$ insulin and $0.02 \mathrm{mg} / \mathrm{ml}$ hydrocortisone (Sigma-Aldrich) for culturing/exposure. After thawing, viability of the cells was checked by a Trypan blue (CAS no. 72-57-1, Sigma-Aldrich) exclusion test as instructed in the supplier's protocol [14]. All viability scores were in accordance with those listed by the supplier. Hepatocytes were exposed for 24 hours to $0,0.2,2$ or $10 \mathrm{mM}$ APAP (CAS no. 103-90-2, Sigma-Aldrich) dissolved in culture medium. The doses, representing a NOEL (No Observed Effect Level), LOEL (Lowest 
Observed Effect Level = non-toxic) and toxic dose respectively were chosen based on available literature [15-24].

\section{Transcriptomic sample preparation}

Total RNA isolation

QIAzol (0.5ml, QIAGEN) was used to isolate total RNA from all samples according to the manufacturer's protocol. RNA purification was performed with the miRNeasy Mini Kit (Qiagen) as instructed by the manufacturer. Next, the integrity of the RNA was checked with the Bioanalyzer 2100 (Agilent).

CDNA preparation/hybridization

From an input of 250ng RNA, cDNA targets were prepared using the Affymetrix protocol. The procedures as recommended by the manufacturer were applied to hybridize the samples to Affymetrix GeneChip Human Genome U133A plus 2 GeneChip arrays. GeneChips were washed and stained after hybridization with a fluidics station (Affymetrix) and scanned with a GeneArray scanner (Affymetrix). The samples from donor 1 exposed to 10mM APAP did not pass quality control and were therefore excluded from further analyses.

\section{Transcriptomic data analysis}

The CEL-files retrieved in the previous step were subjected to an overall quality control, using arrayanalysis.org and all arrays were of high quality [25]. Subsequently, data were RMA-normalized and re-annotated using BrainArray's EntrezGene customCDF_V15.1 [26, 27]. Probes with low signal to noise ratio (average expression $<6$ ) were excluded from further analyses as a data-cleanup step.

\section{Metabolite sample preparation}

Culture medium from the cells was collected after $24 \mathrm{~h}$ and stored at $-80^{\circ} \mathrm{C}$ until extraction. To extract the metabolites, $6 \mathrm{ml}$ of ice-cold acetone was added to $1.5 \mathrm{ml}$ of medium in a $10 \mathrm{ml}$ glass tube. The solution was vortexed for $30 \mathrm{sec}$, kept on ice for $12 \mathrm{~min}$, and then centrifuged for $15 \mathrm{~min}$. at $2800 \mathrm{rpm}$ at $4^{\circ} \mathrm{C}$. The supernatant was transferred into a $10 \mathrm{ml}$ glass tube and dried under nitrogen. To concentrate the semi-polar metabolites contained in the medium, SPE C 18 columns ( $18,500 \mathrm{mg}, 3 \mathrm{ml}$, bond-elut, VARIAN) were used. The SPE C 18 column was conditioned by running it with methanol including $0.5 \%$ of formic acid ( $1 \mathrm{ml}$ twice) and MilliQ including $0.5 \%$ 
of formic acid (1 $\mathrm{ml}$ twice). Once the dried pellet was re-suspended in $1 \mathrm{ml}$ of MilliQ (with $0.5 \%$ formic acid) it was applied to the column. The column was then washed twice with $1 \mathrm{ml}$ of MilliQ, after which the components of interest were eluted with $1 \mathrm{ml}$ of methanol and dried under nitrogen. For U-HPLC-Orbitrap analysis, the dried polar fraction was re-suspended in 400 ul of MilliQ with $0.1 \%$ formic acid.

\section{U-HPLC-Orbitrap-MS analysis}

Experimental set-ups and procedures as described before have been used with some slight modifications as defined below [28-30]. The gradient was similar to the one used in Jetten et al. with small modifications [13]. The initial eluent composition, 100\% A, was changed to $85 \% \mathrm{~A}$ and $15 \% \mathrm{~B}$ in $15 \mathrm{~min}$. Afterwards, the composition of B was increased to $30 \%$ in 10 min and subsequently increased in 3 min to $90 \%$, remaining at this composition for 5 min prior to the next injection. A capillary temperature of $250^{\circ} \mathrm{C}$ with a sheath and auxiliary gas flow of 19 and 7 arbitrary units were used, respectively.

\section{Metabolite data analysis}

Visual inspection of the three technical replicates of each sample showed a high degree of reproducibility. All MS data were pre-processed and aligned using the in-house developed program, metAlign[28]. A targeted search for the metabolites of APAP previously described in Jetten et al. was executed [13]. For targeted analyses, Search LCMS, an add-on tool for metAlign, was used [28]. Briefly, a list of masses of interest was composed based on our previous in vivo study with human volunteers [13] and some further data available from mice [31]. This list was loaded into Search LCMS, which returned the amplitudes of the masses of interest.

U-HPLC-Orbitrap MS data were pre-processed as described in previous papers $[28,29]$ to obtain ultra-precise (sub-ppm) mass data (calibration using internal masses and PEG200, PEG300, PEG600 as external masses). Metabolites were considered to be present when retention times were analogous to earlier experiments and average accurate masses were below +-3 ppm; nearly all average masses of metabolites were within $1 \mathrm{ppm}$. For some metabolites previously not found, including hydroxy-APAP, methoxy-APAP and 3,3'biacetoaminophen, retention times were related to those derived from literature if possible $[13,31]$. Metabolite expression levels for methoxyAPAP-glucuronide1/2 and hydroxy-APAP-glutathione in samples of donor 1 exposed to $10 \mathrm{mM}$ APAP could unfortunately not be determined 
due to technical issues.

\section{Metabolite visualisation}

To create a metabolic map based on available literature a pathway visualization tool, Pathvisio, was used [32-34]. LC-MS data were visualized for each donor and per dose (corrected for control levels, OmM APAP). Log-transformation of the data resulted in range with a minimum of 0 and a maximum of 5 .

\section{Data integration and visualization; Interindividual variation}

Expression data (log2-scaled intensities) of all genes passing the selection as described under 'transcriptomic data analysis' and levels of all of the identified metabolites were plotted against APAP-dose per donor (X-axis: dose 0, 0.2, 2 and 10mM APAP, Y-axis: log scaled gene expression/metabolite levels, line: donor, $n=5$ ) using R 2.15.3 [35]. For clarification purposes a representative plot is provided in Supplementary Figure 1. To estimate the correlation in APAP-dose response between donors, data points from one donor were compared to the data points of all other donors using a Pearson-based correlation analysis, which resulted in the following comparisons: D1-D2, D1-D3, D1-D4, D1-D5, D2-D3, D2-D4, D2-D5, D3-D4, D3-D5 and D4-D5. Then, the absolute correlation coefficients of each donor were summed to generate an arbitrary correlation score per donor for each gene/metabolite (Score D1, Score D2, Score D3, Score D4 and Score D5; see supplementary Figure 1). This 'score' now represents the similarity of a particular donor to all other donors in expression response for a particular gene/ metabolite following APAP exposure; the donor with the lowest score is most aberrant from all other donors (showed the least correlation to the other donors). In order to select the most variable genes between donors, standard deviations (SD) of the donor scores per gene/ metabolite were calculated and ranked. The top-1\% ranked genes (score-SD > 2.52) were selected for further analysis, since these exhibit the most inter-donor variability (Table 1).

To enable biological interpretation, an over-representation analysis was performed on this set of variable genes, using the overrepresentation module of ConsensusPathDB [36]. A background list consisting of all genes passing the selection as described under 'transcriptomic data analysis' was used in this analysis. 
Subsequently, the correlation score matrices (see top left corner supplementary Figure 1) created for each of the top- $1 \%$ highly variable genes and all metabolites, were used to find gene expression profiles that match metabolite profiles on an individual level. To do so, the interdonor correlations for all highly variable genes were correlated to the inter-donor correlations from all metabolites (Pearson). A cut-off of $>0.7$ was used to define genes of which the difference in expression levels mimicked the difference in the metabolite levels between donors. The results of this analysis are summarized in supplementary Table 3.

To define genes related to mitochondrial processes, the top $1 \%$ variable genes were compared to the mitochondrial reference gene set from MITOP2. This is a database which provides a list of human mitochondrial proteins linked to their gene names found through computational prediction of signaling sequences, but also includes results from proteome mapping, mutant screening, expression profiling, protein-protein interaction and cellular sub-localization studies [37]. The MiMl plugin for Cytoscape was used to generate a network for all mitochondrial-related genes based on nearest neighbor analysis (Figure 3) [38]. Only nearest neighbors shared by more than one of the mitochondrial-related genes were taken into consideration.

\section{Results}

\section{Transcriptomics}

Just over 10,000 genes were screened for interindividual variation in their responses towards APAP exposure by correlating their expression over dose. Standard deviations of these correlation scores showed a normal distribution. To assure that only the most variable genes were used for further analyses, a short-list was created of the top-1\% most variable genes (see Table 1, $\mathrm{n}=99, \mathrm{SD}>2.52$ ). 
Table 1; List of top 1\% most variable genes based on Pearson correlation analysis The description of the functionality of the gene has been taken from GeneCards, genes involved in mitochondrial processes according to the MITOP2 database are in italic.

\begin{tabular}{|c|c|c|}
\hline $\begin{array}{l}\text { Entrez- } \\
\text { GenelD }\end{array}$ & Gene name & Functional description according to Genecards \\
\hline 92 & ACVR2A & Kinase receptor \\
\hline 513 & ATP5D & Subunit of mitochondrial ATP synthase \\
\hline 595 & CCND1 & Cyclin family \\
\hline 617 & $B C S 1 L$ & Complex III of the mitochondrial respiratory chain \\
\hline 988 & CDC5L & Cell cycle regulator important for G2/M transition \\
\hline 1545 & CYP1B1 & Cytochrome P450 superfamily \\
\hline 1611 & DAP & Mediator of programmed cell death \\
\hline 2669 & GEM & GTP-binding proteins, receptor-mediated signal transduction \\
\hline 2766 & GMPR & NADPH-dependent reductive deamination of GMP to IMP \\
\hline 3276 & PRMT1 & Methyltransferase \\
\hline 4302 & MLLT6 & Myeloid/Lymphoid Or Mixed-Lineage Leukemia \\
\hline 4615 & MYD88 & Myeloid Differentiation Primary Response \\
\hline 5201 & PFDN1 & Member of the pre-folding beta subunit family \\
\hline 5287 & PIK3C2B & $\begin{array}{l}\text { PI3-kinases play roles in signaling pathways involved in cell proliferation, oncogenic } \\
\text { transformation, cell survival, cell migration, and intracellular protein trafficking }\end{array}$ \\
\hline 5300 & PIN1 & $\begin{array}{l}\text { Regulation of cell growth, genotoxic and other stress responses, the immune } \\
\text { response, induction and maintenance of pluripotency, germ cell development, } \\
\text { neuronal differentiation, and survival }\end{array}$ \\
\hline 5523 & PPP2R3A & Negative control of cell growth and division \\
\hline 5550 & PREP & Maturation and degradation of peptide hormones and neuropeptides \\
\hline 5584 & $\mathrm{PRKCl}$ & $\begin{array}{l}\text { Protective role against apoptotic stimuli, is involved in NF-kappa-B activation, cell } \\
\text { survival, differentiation and polarity, and contributes to the regulation of microtubule } \\
\text { dynamics in the early secretory pathway }\end{array}$ \\
\hline 5696 & PSMB8 & Apoptosis, may be involved in the inflammatory response pathway \\
\hline 5699 & PSMB10 & Involved in antigen processing to generate class I binding peptides \\
\hline 5796 & PTPRK & Cell growth, differentiation, mitotic cycle, and oncogenic transformation \\
\hline 6612 & SUMO3 & $\begin{array}{l}\text { Post-translationally modify numerous cellular proteins and affect their metabolism } \\
\text { and function, such as nuclear transport, transcriptional regulation, apoptosis, and } \\
\text { protein stability" }\end{array}$ \\
\hline 6942 & TCF20 & $\begin{array}{l}\text { Stimulates the activity of various transcriptional activators such as JUN, SP1, PAX6 } \\
\text { and ETS1, suggesting a function as a co-activator }\end{array}$ \\
\hline 7264 & TSTA3 & $\begin{array}{l}\text { Cell-cell interactions, including cell-cell recognition; in cell-matrix interactions; in } \\
\text { detoxification processes }\end{array}$ \\
\hline 7572 & ZNF24 & Transcription repressor activity \\
\hline 7965 & AIMP2 & Functions as a pro-apoptotic factor \\
\hline 8270 & LAGE3 & $? ? ?$ \\
\hline 8310 & ACOX3 & Desaturation of 2-methyl branched fatty acids in peroxisomes \\
\hline
\end{tabular}




\begin{tabular}{|c|c|c|}
\hline 8985 & PLOD3 & Hydroxylation of lysyl residues in collagen-like peptides \\
\hline 9343 & EFTUD2 & $\begin{array}{l}\text { A component of the spliceosome complex which processes precursor mRNAs to } \\
\text { produce mature mRNAs }\end{array}$ \\
\hline 9361 & LONP1 & $\begin{array}{l}\text { Mediates the selective degradation of misfolded, unassembled or oxidatively } \\
\text { damaged polypeptides in the mitochondrial matrix assembly of inner membrane } \\
\text { protein complexes, and participate in the regulation of mitochondrial gene expression } \\
\text { and maintenance of the integrity of the mitochondrial genome }\end{array}$ \\
\hline 9470 & EIF4E2 & $\begin{array}{l}\text { EIF4E2 gene promoter protein synthesis and facilitates ribosome binding by inducing } \\
\text { the unwinding of the mRNAs secondary structures }\end{array}$ \\
\hline 10093 & ARPC4 & $\begin{array}{l}\text { Regulation of actin polymerization and together with an activating nucleation- } \\
\text { promoting factor (NPF) mediates the formation of branched actin networks }\end{array}$ \\
\hline 10189 & THOC4 & $\begin{array}{l}\text { Molecular chaperone. It is thought to regulate dimerization, DNA binding, and } \\
\text { transcriptional activity of basic region-leucine zipper (bZIP) proteins }\end{array}$ \\
\hline 10313 & RTN3 & Involved in membrane trafficking in the early secretory pathway \\
\hline 10422 & UBAC1 & $\begin{array}{l}\text { Required for poly-ubiquitination and proteasome-mediated degradation of CDKN1B } \\
\text { during G1 phase of the cell cycle }\end{array}$ \\
\hline 10598 & AHSA1 & May affect a step in the endoplasmic reticulum to Golgi trafficking \\
\hline 10807 & SDCCAG3 & May be involved in modulation of TNF response \\
\hline 10899 & JTB & $\begin{array}{l}\text { Required for normal cytokinesis during mitosis. Plays a role in the regulation of cell } \\
\text { proliferation }\end{array}$ \\
\hline 11068 & CYB561D2 & $\begin{array}{l}\text { Acting as an ubiquitin conjugating enzyme, involved in the regulation of exit from } \\
\text { mitosis, cell cycle, protein, ubiquitin dependent proteolysis, electron transport }\end{array}$ \\
\hline 11131 & CAPN11 & $\begin{array}{l}\text { Remodeling of cytoskeletal attachments to the plasma membrane during cell } \\
\text { fusion and cell motility, proteolytic modification of molecules in signal transduction } \\
\text { pathways, degradation of enzymes controlling progression through the cell cycle, } \\
\text { regulation of gene expression, substrate degradation in some apoptotic pathways, } \\
\text { and an involvement in long-term potentiation }\end{array}$ \\
\hline 11142 & PKIG & $\begin{array}{l}\text { PKA inhibitors, Protein kinase A has several functions in the cell, including regulation } \\
\text { of glycogen, sugar, and lipid metabolism. }\end{array}$ \\
\hline 11252 & PACSIN2 & $\begin{array}{l}\text { Involved in linking the actin cytoskeleton with vesicle formation by regulating tubulin } \\
\text { polymerization }\end{array}$ \\
\hline 11332 & ACOT7 & $\begin{array}{l}\text { Catalyze the hydrolysis of acyl-CoAs to the free fatty acid and coenzyme } A \text { (COASH), } \\
\text { providing the potential to regulate intracellular levels of acyl-CoAs, free fatty acids } \\
\text { and COASH }\end{array}$ \\
\hline 23243 & ANKRD28 & Involved in the recognition of phosphoprotein substrates \\
\hline 23325 & KIAA1033 & $\begin{array}{l}\text { Plays a key role in the fission of tubules that serve as transport intermediates during } \\
\text { endosome sorting }\end{array}$ \\
\hline 23558 & WBP2 & $\begin{array}{l}\text { Involved in mediating protein-protein interactions through the binding of polyproline } \\
\text { ligands }\end{array}$ \\
\hline 26100 & WIPI2 & $\begin{array}{l}\text { Probable early component of the autophagy machinery being involved in formation } \\
\text { of preautophagosomal structures and their maturation into mature phagosomes }\end{array}$ \\
\hline 26505 & CNNM3 & Probable metal transporter \\
\hline 26520 & TIMM9 & $\begin{array}{l}\text { Mediate the import and insertion of hydrophobic membrane proteins into the } \\
\text { mitochondrial inner membrane }\end{array}$ \\
\hline 27075 & TSPAN13 & $\begin{array}{l}\text { Mediate signal transduction events that play a role in the regulation of cell } \\
\text { development, activation, growth and motility }\end{array}$ \\
\hline 29105 & C16orf80 & $? ? ?$ \\
\hline
\end{tabular}




\begin{tabular}{|c|c|c|}
\hline 29927 & SEC61A1 & $\begin{array}{l}\text { Plays a crucial role in the insertion of secretory and membrane polypeptides into the } \\
\text { ER }\end{array}$ \\
\hline 50640 & PNPLA8 & $\begin{array}{l}\text { Phospholipases which catalyze the cleavage of fatty acids from membrane } \\
\text { phospholipids }\end{array}$ \\
\hline 51094 & ADIPOR1 & Regulates fatty acid catabolism and glucose levels \\
\hline 51491 & NOP16 & Involved in ribosome biogenesis \\
\hline 51504 & TRMT112 & Participates both in methylation of protein and tRNA species \\
\hline 51523 & CXXC5 & $\begin{array}{l}\text { Required for DNA damage-induced ATM phosphorylation, p53 activation and cell } \\
\text { cycle arrest. Involved in myelopoiesis }\end{array}$ \\
\hline 51706 & CYB5R1 & $\begin{array}{l}\text { Involved in desaturation and elongation of fatty acids, cholesterol biosynthesis, drug } \\
\text { metabolism, and, in erythrocyte, methemoglobin reduction }\end{array}$ \\
\hline 54187 & NANS & Functions in the biosynthetic pathways of sialic acids \\
\hline 54606 & DDX56 & $\begin{array}{l}\text { Implicated in a number of cellular processes involving alteration of RNA secondary } \\
\text { structure such as translation initiation, nuclear and mitochondrial splicing, and } \\
\text { ribosome and spliceosome assembly. May play a role in later stages of the processing } \\
\text { of the pre-ribosomal particles leading to mature } 60 \mathrm{~S} \text { ribosomal subunits. Has intrinsic } \\
\text { ATPase activity }\end{array}$ \\
\hline 54941 & RNF125 & E3 ubiquitin-protein ligase that acts as a positive regulator of T-cell activation \\
\hline 55062 & WIPI1 & May play a role in autophagy \\
\hline 55111 & PLEKHJ1 & ??? Phospholipid binding \\
\hline 55238 & SLC38A7 & Mediates sodium-dependent transport of amino acids \\
\hline 55315 & SLC29A3 & Plays a role in cellular uptake of nucleosides, nucleobases, and their related analogs \\
\hline 55647 & RAB20 & Plays a role in the maturation and acidification of phagosomes that engulf pathogens \\
\hline 55700 & MAP7D1 & $\begin{array}{l}\text { Mitotic spindle protein and member of the MAP7 (microtubule-associated protein 7) } \\
\text { family of proteins }\end{array}$ \\
\hline 55743 & CHFR & $\begin{array}{l}\text { Functions in the antephase checkpoint by actively delaying passage into mitosis in } \\
\text { response to microtubule poisons }\end{array}$ \\
\hline 55898 & UNC45A & $\begin{array}{l}\text { Plays a role in cell proliferation and myoblast fusion, binds progesterone receptor and } \\
\text { HSP90, and acts as a regulator of the progesterone receptor chaperoning pathway }\end{array}$ \\
\hline 56005 & C19orf10 & ??? \\
\hline 56267 & CCBL2 & Encodes an aminotransferase that transaminates kynurenine to form kynurenic acid \\
\hline 56910 & STARD7 & ??? \\
\hline 57409 & MIF4GD & Functions in replication-dependent translation of histone mRNAs \\
\hline 64754 & SMYD3 & Histone methyltransferase \\
\hline 64787 & EPS8L2 & $\begin{array}{l}\text { Is thought to link growth factor stimulation to actin organization, generating } \\
\text { functional redundancy in the pathways that regulate actin cytoskeletal remodeling }\end{array}$ \\
\hline 64949 & MRPS26 & $\begin{array}{l}\text { Mammalian mitochondrial ribosomal proteins are encoded by nuclear genes and help } \\
\text { in protein synthesis within the mitochondrion. }\end{array}$ \\
\hline 66036 & MTMR9 & Thought to have a role in the control of cell proliferation \\
\hline 79056 & PRRG4 & ??? Calcium ion binding \\
\hline 80227 & PAAF1 & Involved in regulation of association of proteasome components \\
\hline 80775 & TMEM177 & ??? \\
\hline 89870 & TRIM15 & $? ? ?$ \\
\hline
\end{tabular}




\begin{tabular}{|l|l|l|}
\hline 91663 & MYADM & $\begin{array}{l}\text { ??? Regulates the connection between the plasma membrane and the cortical } \\
\text { cytoskeleton and so can control the endothelial inflammatory response. }\end{array}$ \\
\hline 114971 & PTPMT1 & $\begin{array}{l}\text { Is an essential intermediate in the biosynthetic pathway of cardiolipin, a } \\
\text { mitochondrial-specific phospholipid regulating the membrane integrity and activities } \\
\text { of the organelle }\end{array}$ \\
\hline 124583 & CANT1 & Functions as a calcium-dependent nucleotidase \\
\hline 127687 & C10rf122 & ??? \\
\hline 135932 & TMEM139 & ??? \\
\hline 140465 & MYL6B & Regulatory light chain of myosin \\
\hline 140606 & SELM & $\begin{array}{l}\text { May function as a thiol-disulfide-oxidoreductase that participates in disulfide bond } \\
\text { formation }\end{array}$ \\
\hline 147007 & TMEM199 & ??? \\
\hline 151613 & TTC14 & ??? RNA binding \\
\hline 155066 & ATP6V0E2 & $\begin{array}{l}\text { Play an important role in processes such as receptor-mediated endocytosis, protein } \\
\text { degradation and coupled transport }\end{array}$ \\
\hline 196383 & RILPL2 & $\begin{array}{l}\text { Involved in cell shape and neuronal morphogenesis, positively regulating the } \\
\text { establishment and maintenance of dendritic spines }\end{array}$ \\
\hline 252839 & TMEM9 & May be involved in intracellular transport \\
\hline 253461 & ZBTB38 & May be involved in the differentiation and/or survival of late post-mitotic neurons \\
\hline 375757 & C9orf119 & Required for double-strand break repair via homologous recombination \\
\hline 389203 & C4Orf52 & ??? \\
\hline 100128750 & LOC100128750 & ??? \\
\hline 100505687 & LOC100505687 & ??? \\
\hline
\end{tabular}

To define the functionality of the variable genes, an overrepresentation analysis was performed i.e. a network consisting of the biological pathways containing the genes with the highest variability between donors in response to APAP-exposure was defined (see Figure 1). This over-representation network could be broken down into several parts; one large component appeared constructed of:

- A large cluster with toll-like receptors (TLR), c-Jun N-terminal kinases (JNK), nuclear factor (NF)-KB, interleukin (IL)-1, p38 and Cyclin D1-related pathways (encircled with striped line).

- A smaller cluster around p75 NeuroTrophin Receptor (p75NTR) (encircled with dotted line).

- Two more components, involved in 'Wnt-signaling pathway and pluripotency' and 'BTG (B-cell Translocation Gene) family proteins and cell cycle regulation'.

Additional parts consisted of components separated from the large cluster on 'Leukotriene metabolism', 'Biosynthesis of unsaturated fatty acids', 'Amino sugar and nucleotide sugar metabolism' and 
'Retinoic acid-inducible gene 1 (RIG-I)- Melanoma DifferentiationAssociated protein 5 (MDA5) mediated induction of Interferon (IFN)alpha-beta'.

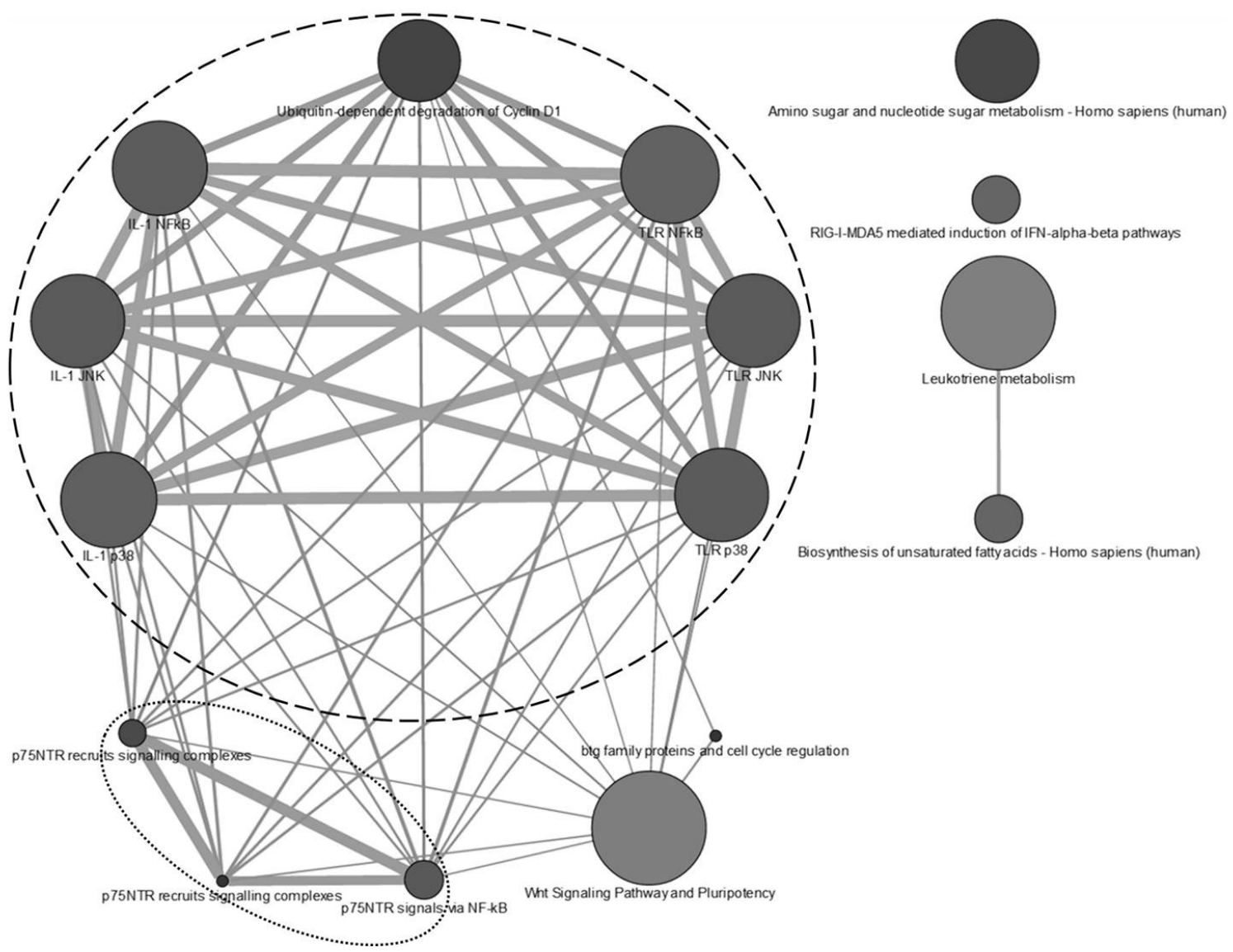

Figure 1; Network of top $1 \%$ most variable genes between donors after APAP-exposure The network was created based on a gene set over-representation analysis (ConsensusPathDB). Each node represents a biological pathway, the size of the node represents the amount of genes included in the pathway (bigger diameter = larger \# genes), the color of the node represents its significance (darker gray = lower p-value) and the thickness of the edge represents the amount of overlap between the connected nodes (thicker line = higher \# overlapping genes).

A further network based on mitochondrial-related genes form the top-1\% highly variable genes was created using next neighbor analysis in Cytoscape (Figure 2). This figure shows that transcription factors are the main binding element in the response of mitochondrialrelated genes showing high interindividual variation in gene expression response after APAP-exposure. 


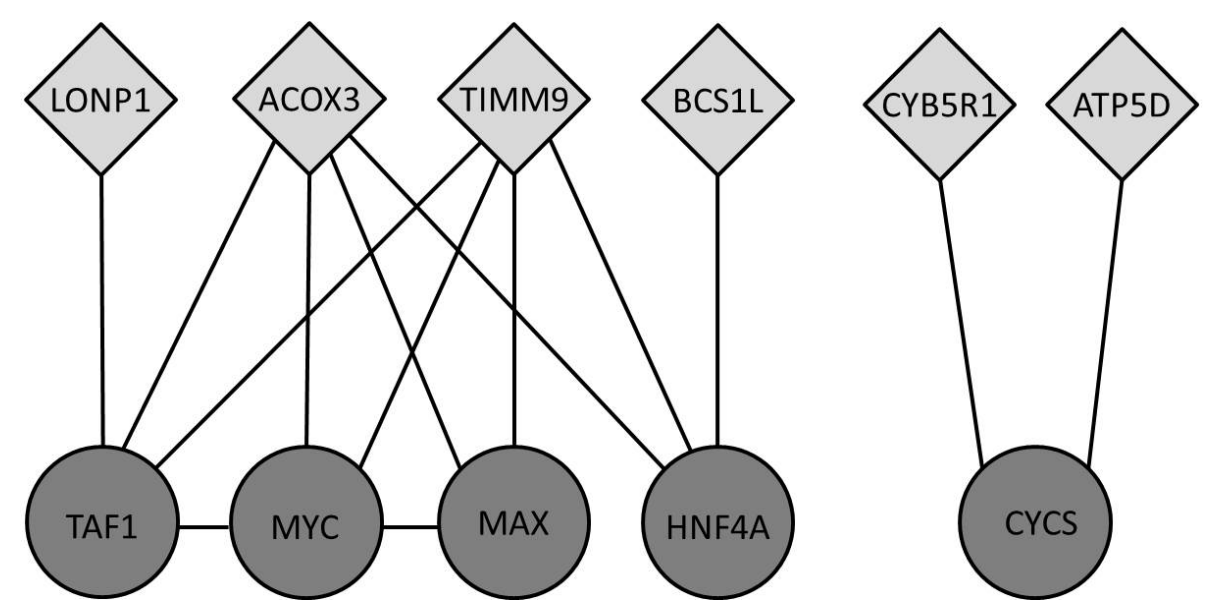

Figure 2; network of highly variable mitochondrial-related genes

Nearest neighbor analysis was performed on all mitochondrial related genes from the top-1\% highly variable gene list. Only nearest neighbors shared by more than one of the variable gene were taken into account. Square nodes represent input genes, round nodes represent shared nearest neighbors.

\section{Metabolites}

A broad spectrum of metabolites was measured in the medium, as shown in Supplementary Table 3 and Figure 3. In general the variation between individuals was lower with respect to metabolite levels when compared to the variation in gene expression levels. To define how the variability between donors in gene expression is related to the variability in metabolite level in these same donors, a Pearsonbased correlation analysis between the top-1\% variable genes and all metabolites was performed (cut-off R2>0.7). Out of the 99 most variable genes, 91 could be linked to the variation in metabolites on an individual level, meaning that these 91 genes can at least partially explain the interindividual variation observed in metabolites. Especially hydroxy-APAP, methoxy-APAP and the tentatively identified metabolite C8H1305N -APAP-glucuronide showed strong correlations with genes on an individual level ( $n=36,36$ and 51 correlating genes respectively). Interestingly, $\mathrm{C} 8 \mathrm{H} 1305 \mathrm{~N}$-APAP-glucuronide has previously been reported by Jetten et al. as a novel APAP-metabolite, which could be detected in the in vivo human situation after low-dose APAP exposure [13]. This metabolite could thus be confirmed in the current study in an in vitro human situation consisting of primary human hepatocytes. Furthermore, a mass tentatively assigned to 3,3'-biacetaminophen (not detected previously) has also been found. 3,3'-biacetaminophen has been suggested to result from NAPQI reacting with APAP and is considered a reactive oxygen species (ROS) product [31]. 
APAP-

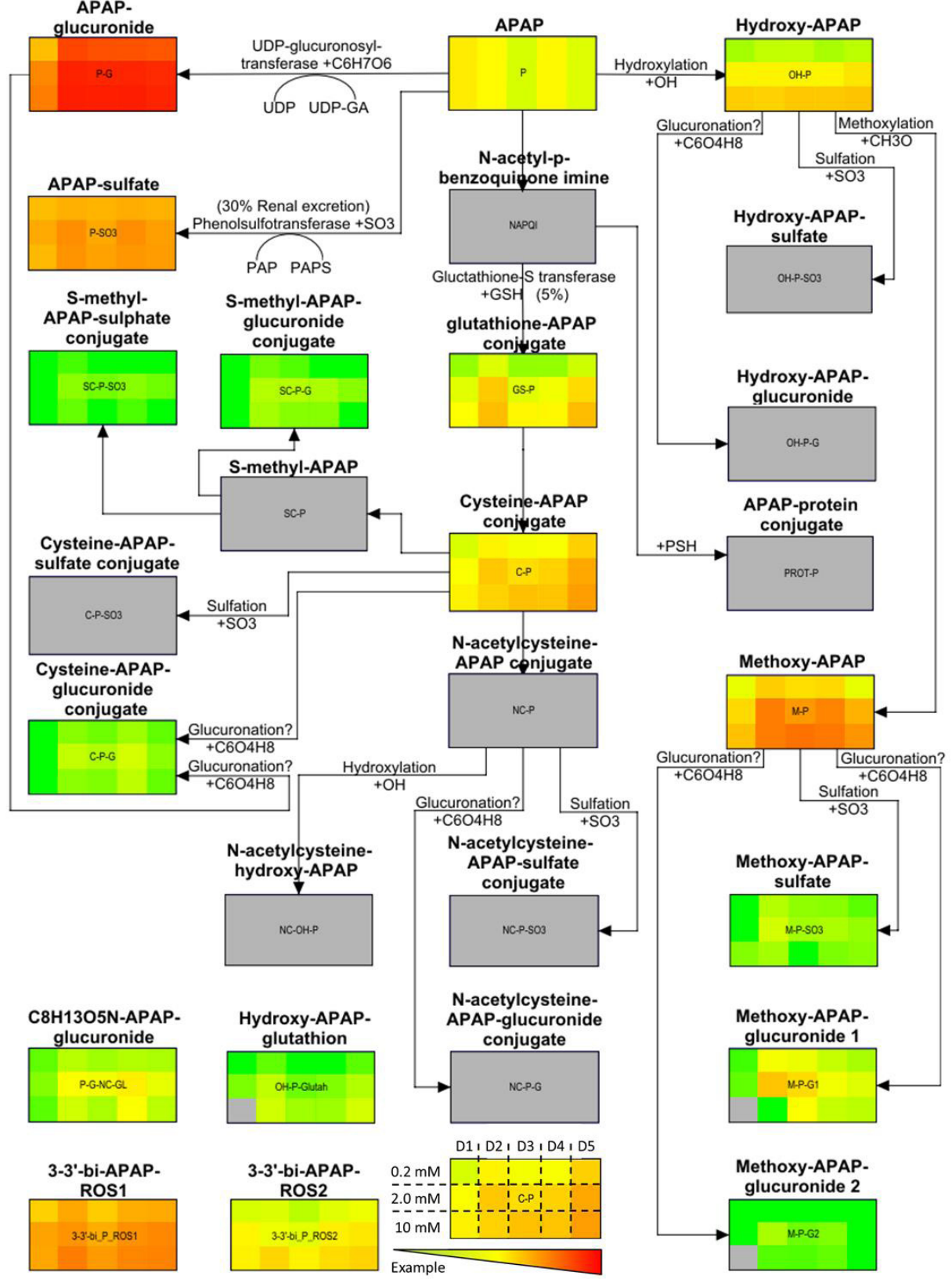

Figure 3; Schematic visualization of APAP metabolic pathway

The log-transformed metabolite levels for each donor on at each dose corrected for OmM are visualized. Gray boxes; not measured/detected. Increase in a metabolite is pictured from green (no increase, equals a numerical value of 0 on a log-scale) to yellow, orange and red (high increase, maximum value equals 5 on a log-scale). Figure adapted from Jetten et al [13]. 
The aim of this study is to evaluate the interindividual differences in gene expression changes and APAP-metabolite formation in primary human hepatocytes of several donors $(n=5)$ exposed to a non-toxic to toxic APAP dose-range.

Interindividual variation in gene expression is a very common phenomenon, therefore we have focused on the gene expression changes that are most different between individuals in response to APAP-exposure. To do so, we have created a shortlist consisting of the top $1 \%$ most different genes based on correlation analysis $(n=99$, see Table 1). In general, interindividual differences in responses to APAP, including variance in toxicity outcome, metabolite formation and susceptibility to liver failure. Expression levels of many genes/ metabolites including, but not limited to cytochrome P450 enzymes, glucuronosyltransferases, sulfotransferases and glutathione S-transferases have been shown to influence the biotransformation processes of APAP [39]. However, studies in general link baseline expression levels of these genes to APAP-metabolism parameters, while in the current study we focus on response parameters after APAPexposure in order to explain inter-individual variability.

To define the biological functionality of the genes with the highest variability between individuals (top-1\% list), a network of pathways found by gene set over-representation analysis on this list was created (Figure 1). This network shows a large cluster with TLRs, JNK, NF-KB, IL-1, p38 and Cyclin D1-related pathways (encircled with striped line). TLR, JNK and NFKB pathways are all key regulators in the production of cytokines associated with inflammatory responses and the early stages of the development of hepatocarcinogenesis [40]. Furthermore, all of the above mentioned pathways have been associated with the process of liver regeneration [41]. Hepatocytes rarely undergo proliferation in the liver under normal circumstances. However, proliferation can be triggered in response to loss of liver mass for instance induced by liver resection but also due to toxin-induced hepatocyte trauma like is the case with APAP. In both in vitro and in vivo studies, APAP has been show to induce a persistent activation of JNK adding to hepatocellular necrosis [42]. Cross-talk between JNK and NF-KB has been proposed as a mechanism through which JNK mediates cell death. Matsumura et al. showed that inhibition of NF-KB in the liver by APAP leads to amplification of JNK and as such shifts the balance from cell survival towards cell death [43]. IL-1 stimulates both the JNK 
and NFKB pathways, which increases cell signaling and interferes with the cell cycle $[44,45]$. TLR plays a role in the expression of cytokines and hepatomitogens, in response to TRL activation, p38 is triggered leading to cytokine- and stress-induced apoptosis [44, 45]. Finally, it is indicated that that NF-KB activation operates on cell growth through Cyclin D1 expression [40,46]. In acetaminophen-induced liver injury, sustained JNK activation, through NF-KB, is essential in inducing apoptosis [47]. Furthermore it is suggested that NAPQI-induced damage on its own is not enough to cause hepatocyte death after APAP dosing, and that activation of signaling pathways involving JNK are necessary to lead to cell death $[47,48]$. The actual downstream targets of JNK that are involved in APAP-induced liver injury are still largely unknown, however a role for mitochondrial proteins has recently been suggested [49].

Interestingly, $10 \%$ of the genes from the top-1\% highly variable gene list consists of mitochondrial-related genes (see Table1; genes in Italic). The majority of these genes are involved in metabolism related processes (CCBL2 [50], PTPMT1 [51], ACOX3 [52], ACOT7 [53] and CYB5R1 [54]), while others are part of the respiratory chain complexes in the mitochondria (ATP5D [55] and BCS1L [56]), or are involved in more structurally related like protein synthesis (MRPS26 [55]) and mitochondrial matrix or membrane maintenance (LONP1 [57] and TIMM9 [55] respectively). All but one (MRPS26) of the above mentioned genes has been related to the toxic/necrotic effects of APAP in a study comparing the toxicity response to APAP in rats/mice with the response to APAP's far less toxic stereo-isomer $\mathrm{N}$-acetyl-m-aminophenol (AMAP) [58]. In addition, ATP5D, MRPS26, LONP1, ACOT7 and TIMM9 were also shown to be regulated in HepG2 cells exposed to a toxic $(10 \mathrm{mM})$ APAP-dose for 12-72 hours (unpublished results). Drug-induced liver injury has often been linked to regulation of the mitochondrial stress responses, which include the formation of ROS products, alterations in lipid metabolism, electron transport, cofactor metabolism and the activation of pathways important in determining cell survival or death $[58,59]$.

Nearest neighbor analyses on all mitochondrial-related genes from the top-1\% highly variable gene list shows that mainly transcription factors seem to be the binding element in the response of the mitochondrial-related genes (Figure 2). The involvement of transcription factors has been suggested in the drug-induced stress response of mitochondria in hepatocytes [59]. This indicates that interindividual variation exists in the response of mitochondrialrelated genes, possibly explaining part of the differences between 
humans in mitochondrial-related APAP-induced toxicity responses and consequential liver damage levels.

Furthermore, in the gene set over-representation network another smaller network around p75NTR is present (Figure 1, encircled with dotted line). P75NTR is a cell membrane receptor protein that has been associated with tumor and metastasis suppression [60], similar to the cluster described above. Non-steroidal anti-inflammatory drugs (NSAIDs) are used to reduce inflammation and also act as analgesics by the inhibition of cyclooxygenase-2 (COX-2). However, high concentrations of some NSAIDs are able to reduce proliferation and induce apoptosis in cancer cells. Several molecular mechanisms have been proposed as possible mediators in the anti-cancerous effects of NSAIDs, including p75NTR. Although APAP is not considered to be a real NSAID, due to its limited anti-inflammatory effects, APAP does affect similar pathways and works as an analgesic through COX-2 inhibition which might explain why similar effects have been suggested for APAP [61].

Two other components in the gene set over-representation network that are connected to the components described above are the 'Wnt-signaling pathway and pluripotency' and 'BTG family proteins and cell cycle regulation' (see Figure 1). Both pathways can be related to APAP- induced toxicity related effects. The stimulation of the Wntpathway has been suggested to be beneficial after APAP-induced liver failure by stimulating liver regeneration [62]. This corresponds with the previously mentioned regulation of liver regeneration by the genes involved in the cluster around TLRs, JNK, NF-KB, IL-1, p38 and Cyclin D1-related pathways. The BGT gene-family has been associated with APAP hepatotoxicity before [58] and has a function in DNA-strand break repair [63] and in the regulation of reactive oxygen species generation in the mitochondria [27]. Both APAP and NAPQI are known to covalently bind to DNA and cause DNA-damage $[64,65]$, which possibly explains why this pathway is triggered by APAP-exposure. As already described above, mitochondrial stress is an intricate part of the cellular response to APAP-induced oxidative stress [47], which might also explain the response of the BTG-related pathway. These findings agree with the previously mentioned interindividual variation in mitochondrial genes.

In addition, several other pathways not connected to the main cluster of pathways are included in the network of the gene set overrepresentation analysis. These are 'Leukotriene metabolism' together with 'Biosynthesis of unsaturated fatty acids', 'Amino sugar and 
nucleotide sugar metabolism' and 'RIG-I-MDA5 mediated induction of IFN-alpha-beta'. The first set of pathways (leukotrienes and fatty acids), probably represents the variation in the normal, therapeutic mechanism of APAP. In humans unsaturated fatty acids are bio-activated through enzymatic oxygenation to, amongst others, prostaglandins and leukotrienes which contribute to fever, pain, inflammation, and cancer development [66]. APAP interferes with these processes as such inhibiting symptoms. Concerning the sugar metabolism pathway, carbohydrate homeostasis is essential for normal liver function. It is well-known that during APAP-induced liver failure these processes are severely affected [67]. Finally, RIG-I-MDA5 mediated induction of IFNalpha-beta is a process that has been linked to several liver pathologies like hepatitis A/B/C and hepatic steatosis [68-70]. Although no apparent link with APAP is available in literature, it seems that this process is somehow linked to an APAP-induced stress response.

To determine how the variation in the above mentioned genes can explain the interindividual variation in metabolism levels, a correlation analysis was performed between the top-1\% most variable genes and all metabolites. Glucuronidation and sulfation are the 2 major processes in APAP-metabolism, resulting in APAP-glucuronide and APAP-sulfate, respectively, which are non-toxic APAP-metabolites (see Figure 3) [31]. In addition, another less abundant route of APAPmetabolism utilizes hydroxylation/methoxylation of APAP. Hydroxy-APAP and methoxy-APAP are oxidative metabolites formed during this route of APAP-metabolism, and both these metabolites have been associated with the hepatotoxic effects of APAP $[31,71,72]$. The variation in both these metabolites could be explained by a large proportion of the genes from the top- $1 \%$ most variable genes ( $n=36$ for both metabolites). It thus seems that the largest interindividual variation in gene expression responses after APAP-exposure can be linked to the formation of toxic APAP-metabolite formation. Interestingly, hydroxy/methoxy-derived metabolites could also be detected in human in vivo low-dose APAP exposure [13]. The fact that these metabolites and their derivatives are detectable both in vivo and in vitro, even at APAP-doses within the therapeutic range, and that their variation in expression levels between individuals can be linked to the variation in gene expression of genes related to toxicity related effects of APAP-exposure, indicates a role as potential key-elements in the molecular mechanism behind APAP-toxicity. Additionally, another metabolite, C8H1305N-APAPglucuronide, also shows correlation to a large set of the top-1\% variable genes $(n=51)$. This metabolite was described as new in the low-dose in vivo APAP-exposure study of Jetten et al [13]. Since this metabolite is 
relatively unknown, further studies on its exact route of metabolism and toxic potency could lead to further insight in the molecular mechanism behind APAP-toxicity for the same reasons as explained for hydroxyl/ methoxy-APAP above.

In summary, the biological processes in which the genes with the highest variability in expression between individuals after APAPexposure are involved can be linked to APAP-toxicity related processes like liver regeneration, inflammatory responses, and mitochondrial stress responses. Also, processes related to hepatocarcinogenesis, cell cycle and drug-efficacy show large interindividual variation after APAPexposure. In addition, most of the genes with high variability between individuals after APAP-exposure can be linked to variability in expression levels of metabolites (hydroxyl/methoxy-APAP and C8H13O5NAPAP-glucuronide). Possibly these findings could help explain the differences seen in susceptibility to APAP-toxicity in the in vivo situation. Furthermore, they might give an indication for where the factors causing variability in susceptibility towards APAP-induced liver failure could be found.

\section{Acknowledgements}

This work was supported by the Dutch Ministry of Public Health, Welfare and Sports (VWS) as a part of the Assuring Safety without Animal Testing (ASAT) initiative. 


\section{References}

1. Lee, W.M., Acetaminophen toxicity: changing perceptions on a social/medical issue. Hepatology, 2007. 46(4): p. 966-70.

2. Dahlin, D.C., et al., N-acetyl-p-benzoquinone imine: a cytochrome P-450mediated oxidation product of acetaminophen. Proc Natl Acad Sci U S A, 1984. 81(5): p. 1327-31.

3. Jaeschke, H., M.R. McGill, and A. Ramachandran, Oxidant stress, mitochondria, and cell death mechanisms in drug-induced liver injury: lessons learned from acetaminophen hepatotoxicity. Drug Metab Rev, 2012. 44(1): p. 88-106.

4. Hinson, J.A., D.W. Roberts, and L.P. James, Mechanisms of acetaminopheninduced liver necrosis. Handbook of experimental pharmacology, 2010(196): p. 369-405.

5. Greek, R. and A. Menache, Systematic Reviews of Animal Models: Methodology versus Epistemology. International journal of medical sciences, 2013. 10(3): p. 206-21.

6. Court, M.H., et al., Interindividual variability in acetaminophen glucuronidation by human liver microsomes: identification of relevant acetaminophen UDPglucuronosyltransferase isoforms. J Pharmacol Exp Ther, 2001. 299(3): p. 998-1006.

7. Berg, E.L., Systems biology in drug discovery and development. Drug Discovery Today, 2014. 19(2): p. 113-125.

8. Yasar, U., et al., Evidence for regulation of UDP-glucuronosyltransferase (UGT) $1 \mathrm{~A} 1$ protein expression and activity via DNA methylation in healthy human livers. The Journal of pharmacy and pharmacology, 2013. 65(6): p. 874-83.

9. Fisher, M.B., et al., Tissue distribution and interindividual variation in human UDP-glucuronosyltransferase activity: relationship between UGT1A1 promoter genotype and variability in a liver bank. Pharmacogenetics, 2000. 10(8): p. 727-39.

10. Polasek, T.M., D.J. Elliot, and J.O. Miners, Measurement of human cytochrome P4501A2 (CYP1A2) activity in vitro. Curr Protoc Toxicol, 2006. 4(19).

11. Sabate, M., et al., Paracetamol in therapeutic dosages and acute liver injury: causality assessment in a prospective case series. BMC Gastroenterol, 2011. 11: p. 80.

12. Turkoski, B.B., Acetaminophen: old friend--new rules. Orthop Nurs, 2010. 29(1): p. 41-3; quiz 44-5.

13. Jetten, M.J., et al., 'Omics analysis of low dose acetaminophen intake demonstrates novel response pathways in humans. Toxicology and applied pharmacology, 2012. 259(3): p. 320-8.

14. Invitrogen, No Standard Like a Gold Standard - InvitrogenTM Hepatic Biology Products. Invitrogen product brochure, 2009: p. 11-15.

15. Bannwarth, B., et al., Single and multiple dose pharmacokinetics of acetaminophen (paracetamol) in polymedicated very old patients with rheumatic pain. The Journal of Rheumatology, 2001. 28(1): p. 182-184.

16. Borin, M.T. and J.W. Ayres, Single dose bioavailability of acetaminophen following oral administration. International Journal of Pharmaceutics, 1989. 54(3): p. 199-209.

17. Critchley, J.A.J.H., et al., Differences in the single-oral-dose pharmacokinetics and urinary excretion of paracetamol and its conjugates between Hong Kong Chinese and Caucasian subjects. Journal of Clinical Pharmacy and Therapeutics, 2005. 30(2): p. 179-184.

18. Douglas, D.R., J.B. Sholar, and M.J. Smilkstein, A pharmacokinetic comparison 
of acetaminophen products (Tylenol Extended Relief vs regular Tylenol). Acad Emerg Med, 1996. 3(8): p. 740-4.

19. Kamali, F., The effect of probenecid on paracetamol metabolism and pharmacokinetics. European Journal of Clinical Pharmacology, 1993. 45(6): p. 551-553.

20. Kienhuis, A.S., et al., Parallelogram approach using rat-human in vitro and rat in vivo toxicogenomics predicts acetaminophen-induced hepatotoxicity in humans. Toxicol Sci, 2009. 107(2): p. 544-52.

21. Portolés, A., et al., A new high-absorption-rate Paracetamol 500-mg formulation: a comparative bioavailability study in healthy volunteers. Current Therapeutic Research, 2003. 64(7): p. 401-411.

22. Rygnestad, T., K. Zahlsen, and F.A. Samdal, Absorption of effervescent paracetamol tablets relative to ordinary paracetamol tablets in healthy volunteers. European Journal of Clinical Pharmacology, 2000. 56(2):

p. 141-143.

23. Tan, C. and A. Graudins, Comparative pharmacokinetics of Panadol Extend and immediate-release paracetamol in a simulated overdose model. Emerg Med Australas, 2006. 18(4): p. 398-403.

24. Yin, O.Q.P., et al., Pharmacokinetics of acetaminophen in Hong Kong Chinese subjects. International Journal of Pharmaceutics, 2001. 222(2): p. 305-308.

25. Eijssen, L.M., et al., User-friendly solutions for microarray quality control and pre-processing on ArrayAnalysis.org. Nucleic acids research, 2013. 41(Web Server issue): p. W71-6.

26. Dai, M., et al., Evolving gene/transcript definitions significantly alter the interpretation of GeneChip data. Nucleic acids research, 2005. 33(20): p. e175.

27. Lim, S.K., et al., BTG2 suppresses cancer cell migration through inhibition of Src-FAK signaling by downregulation of reactive oxygen species generation in mitochondria. Clinical \& experimental metastasis, 2012. 29(8): p. 901-13.

28. Lommen, A., MetAlign: interface-driven, versatile metabolomics tool for hyphenated full-scan mass spectrometry data preprocessing. Analytical chemistry, 2009. 81(8): p. 3079-86.

29. Lommen, A., et al., Ultra-fast searching assists in evaluating sub-ppm mass accuracy enhancement in U-HPLC/Orbitrap MS data. Metabolomics : Official journal of the Metabolomic Society, 2011. 7(1): p. 15-24.

30. Ruiz-Aracama, A., et al., An untargeted multi-technique metabolomics approach to studying intracellular metabolites of HepG2 cells exposed to 2,3,7,8-tetrachlorodibenzo-p-dioxin. BMC genomics, 2011. 12: p. 251.

31. Chen, C., et al., Identification of novel toxicity-associated metabolites by metabolomics and mass isotopomer analysis of acetaminophen metabolism in wild-type and Cyp2e1-null mice. The Journal of biological chemistry, 2008. 283(8): p. 4543-59.

32. van lersel, M.P., et al., Presenting and exploring biological pathways with PathVisio. BMC Bioinformatics, 2008. 9: p. 399.

33. Chen, $C$., et al., Identification of novel toxicity-associated metabolites by metabolomics and mass isotopomer analysis of acetaminophen metabolism in wild-type and Cyp2e1-null mice. J Biol Chem, 2008. 283(8): p. 4543-59.

34. Daykin, C.A., et al., The comparison of plasma deproteinization methods for the detection of low-molecular-weight metabolites by (1)H nuclear magnetic resonance spectroscopy. Anal Biochem, 2002. 304(2): p. 220-30.

35. R-Core-Team. R: A language and environment for statistical computing. 2013; Available from: http://www.R-project.org/.

36. Kamburov, A., et al., The ConsensusPathDB interaction database: 2013 update. 
Nucleic acids research, 2013. 41(Database issue): p. D793-800.

37. Elstner, M., et al., The mitochondrial proteome database: MitoP2. Methods Enzymol, 2009. 457: p. 3-20.

38. Gao, J., et al., Integrating and annotating the interactome using the MiMI plugin for cytoscape. Bioinformatics, 2009. 25(1): p. 137-8.

39. Zhao, L. and G. Pickering, Paracetamol metabolism and related genetic differences. Drug metabolism reviews, 2011. 43(1): p. 41-52.

40. Maeda, S., NF-kappaB, JNK, and TLR Signaling Pathways in Hepatocarcinogenesis. Gastroenterology research and practice, 2010. 2010: p. 367694.

41. limuro, Y. and J. Fujimoto, TLRs, NF-kB, JNK, and Liver Regeneration. Gastroenterology research and practice, 2010. 2010.

42. Gunawan, B.K., et al., c-Jun N-terminal kinase plays a major role in murine acetaminophen hepatotoxicity. Gastroenterology, 2006. 131(1): p. 165-78.

43. Matsumura, T., et al., TRAF6-NF-kappaB pathway is essential for interleukin1-induced TLR2 expression and its functional response to TLR2 ligand in murine hepatocytes. Immunology, 2003. 109(1): p. 127-36.

44. Matsuzawa, A., et al., ROS-dependent activation of the TRAF6-ASK1-p38 pathway is selectively required for TLR4-mediated innate immunity. Nature immunology, 2005. 6(6): p. 587-92.

45. Sanz-Garcia, C., et al., Sterile inflammation in acetaminophen-induced liver injury is mediated by Cot/tpl2. The Journal of biological chemistry, 2013. 288(21): p. 15342-51.

46. Guttridge, D.C., et al., NF-kappaB controls cell growth and differentiation through transcriptional regulation of cyclin D1. Molecular and cellular biology, 1999. 19(8): p. 5785-99.

47. Hanawa, N., et al., Role of JNK translocation to mitochondria leading to inhibition of mitochondria bioenergetics in acetaminophen-induced liver injury. The Journal of biological chemistry, 2008. 283(20): p. 13565-77.

48. Kaplowitz, N., Idiosyncratic drug hepatotoxicity. Nature reviews. Drug discovery, 2005. 4(6): p. 489-99.

49. Zhou, Q., et al., c-Jun N-terminal kinase regulates mitochondrial bioenergetics by modulating pyruvate dehydrogenase activity in primary cortical neurons. Journal of neurochemistry, 2008. 104(2): p. 325-35.

50. Yu, P., et al., Characterization of kynurenine aminotransferase III, a novel member of a phylogenetically conserved KAT family. Gene, 2006. 365(0): p. 111-118.

51. Shen, J., et al., A critical role of mitochondrial phosphatase Ptpmt1 in embryogenesis reveals a mitochondrial metabolic stress-induced differentiation checkpoint in embryonic stem cells. Molecular and cellular biology, 2011. 31(24): p. 4902-16.

52. Cui, Z., et al., The profile of mitochondrial proteins and their phosphorylation signaling network in INS-1 beta cells. Journal of proteome research, 2010. 9(6): p. 2898-908.

53. Fujita, M., et al., Upregulation of fatty acyl-CoA thioesterases in the heart and skeletal muscle of rats fed a high-fat diet. Biological \& pharmaceutical bulletin, 2011. 34(1): p. 87-91.

54. Chae, Y.C., et al., Landscape of the mitochondrial Hsp90 metabolome in tumours. Nature communications, 2013. 4: p. 2139.

55. Sotgia, F., et al., Mitochondria "fuel" breast cancer metabolism: fifteen markers of mitochondrial biogenesis label epithelial cancer cells, but are excluded from adjacent stromal cells. Cell cycle, 2012. 11(23): p. 4390-401. 
56. Kotarsky, H., et al., Metabolite profiles reveal energy failure and impaired beta-oxidation in liver of mice with complex III deficiency due to a BCS1L mutation. PloS one, 2012. 7(7): p. e41156.

57. Tian, Q., et al., Lon peptidase 1 (LONP1)-dependent breakdown of mitochondrial 5-aminolevulinic acid synthase protein by heme in human liver cells. The Journal of biological chemistry, 2011. 286(30): p. 26424-30.

58. Beyer, R.P., et al., Multicenter study of acetaminophen hepatotoxicity reveals the importance of biological endpoints in genomic analyses. Toxicol Sci, 2007. 99(1): p. 326-37.

59. Han, D., et al., Regulation of drug-induced liver injury by signal transduction pathways: critical role of mitochondria. Trends Pharmacol Sci, 2013. 34(4): p. 243-53.

60. Khwaja, F., et al., Ibuprofen inhibits survival of bladder cancer cells by induced expression of the p75NTR tumor suppressor protein. Cancer research, 2004. 64(17): p. 6207-13.

61. Bonnefont, J., et al., Acetaminophen recruits spinal p42/p44 MAPKs and $\mathrm{GH} / \mathrm{IGF}-1$ receptors to produce analgesia via the serotonergic system. Molecular pharmacology, 2007. 71(2): p. 407-15.

62. Apte, U., et al., Beta-catenin activation promotes liver regeneration after acetaminophen-induced injury. The American journal of pathology, 2009. 175(3): p. 1056-65.

63. Choi, K.S., et al., TIS21(/BTG2/PC3) accelerates the repair of DNA double strand breaks by enhancing Mre11 methylation and blocking damage signal transfer to the Chk2(T68)-p53(S20) pathway. DNA repair, 2012. 11(12): p. 965-75.

64. Dybing, E., et al., Genotoxicity studies with paracetamol. Mutation research, 1984. 138(1): p. 21-32.

65. Rannug, U., et al., International Commission for Protection against Environmental Mutagens and Carcinogens. An evaluation of the genetic toxicity of paracetamol. Mutation research, 1995. 327(1-2): p. 179-200.

66. Ricciotti, E. and G.A. FitzGerald, Prostaglandins and inflammation. Arteriosclerosis, thrombosis, and vascular biology, 2011. 31(5): p. 986-1000.

67. Record, C.O., et al., Disturbances in glucose metabolism in patients with liver damage due to paracetamol overdose. Clin Sci Mol Med, 1975. 49(5): p. 473-9.

68. Kawai, T., et al., IPS-1, an adaptor triggering RIG-I- and Mda5-mediated type I interferon induction. Nature immunology, 2005. 6(10): p. 981-8.

69. Toyoda, H., et al., Association between hepatic steatosis and hepatic expression of genes involved in innate immunity in patients with chronic hepatitis $C$. Cytokine, 2013. 63(2): p. 145-50.

70. Wei, $C$., et al., The hepatitis $B$ virus $X$ protein disrupts innate immunity by downregulating mitochondrial antiviral signaling protein. Journal of immunology, 2010. 185(2): p. 1158-68.

71. Dahlin, D.C., et al., N-acetyl-p-benzoquinone imine: a cytochrome P-450mediated oxidation product of acetaminophen. Proceedings of the National Academy of Sciences of the United States of America, 1984. 81(5): p. 1327-31.

72. Wilson, J.M., et al., Analysis of acetaminophen metabolites in urine by highperformance liquid chromatography with UV and amperometric detection. Journal of chromatography, 1982. 227(2): p. 453-62. 



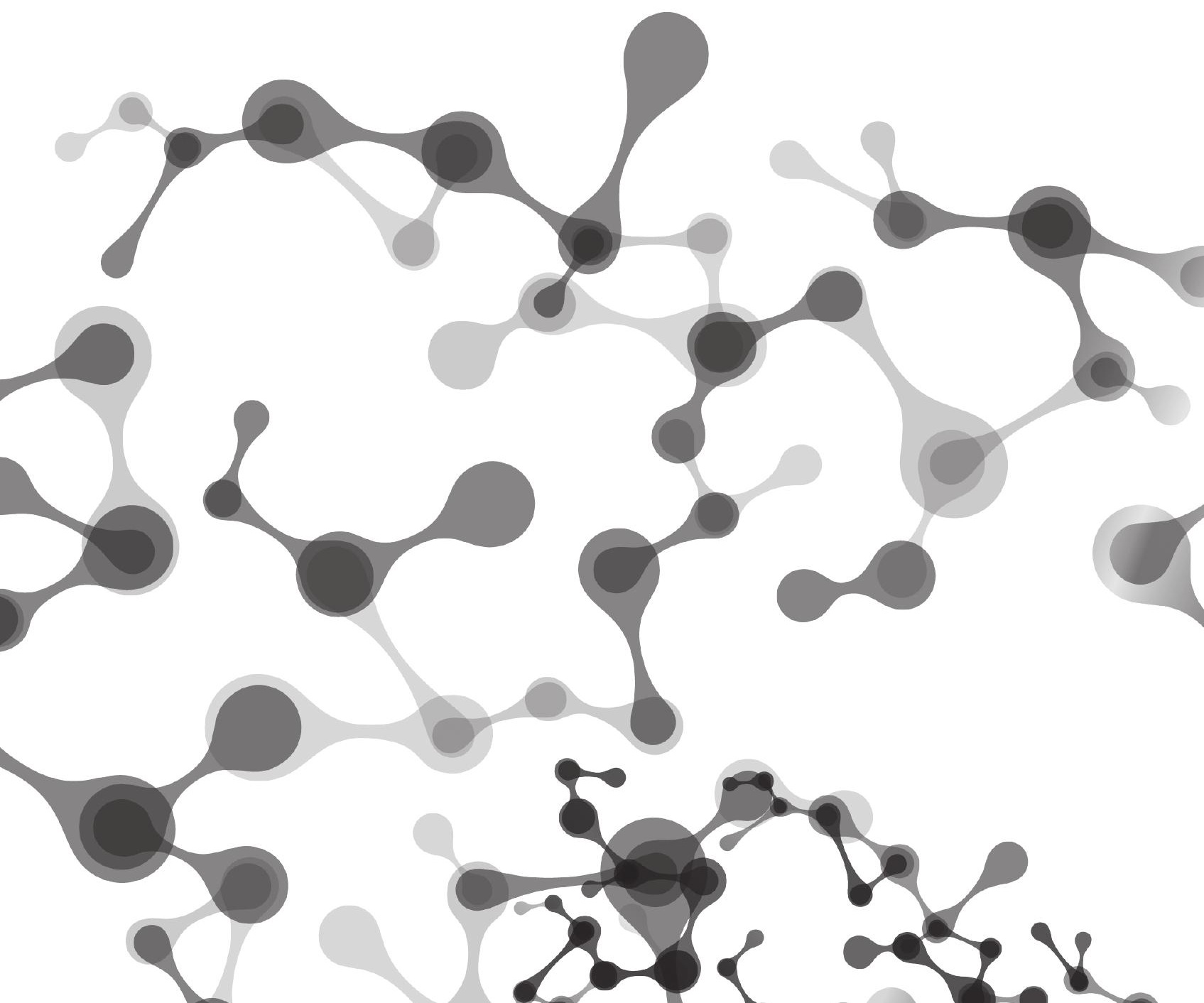




\section{Chapter 4}

\section{Baseline and genotoxic compound induced gene expression profiles in HepG2 and HepaRG compared to primary human hepatocytes}

Marlon J.A. Jetten Jos C.S. Kleinjans

Sandra M. Claessen

Christophe Chesné Joost H.M. van Delft

Published: Toxicology In Vitro: Volume 27, Issue 7, October 2013, Pages 2031-2040 


\section{Abstract}

Efforts are put into developing toxicogenomics-based toxicity testing methods using in vitro human cell models for improving human risk assessment/replacing animal models. Human in vitro liver models include HepG2, HepaRG and primary human hepatocytes (PHH). Studies on comparability/applicability of these cell types mainly focus on assessing baseline biotransformation capacities/cytochrome P450-inducibility, but compound-induced gene expression profiles are at least as important.

Therefore, we compared baseline and aflatoxin B1- and benzo( $\alpha)$ pyrene-induced gene expression profiles in HepG2, HepaRG and PHH (11-13 donors).

At baseline, all liver models differ from each other with respect to whole genome gene expression levels. $\mathrm{PHH}$ show profound interindividual differences, and are most similar to HepaRG. After compound exposure, induced gene expression profiles are more similar between cell models, especially for benzo( $\alpha$ )pyrene. Pathways involved in compound metabolism are induced in all 3 models, while others are more pronounced in a specific cell model. Examples are transcriptomic modifications of carbohydrate-related genes (HepaRG) and of receptorrelated genes $(\mathrm{PHH})$ after benzo( $\alpha)$ pyrene exposure, and of cell cyclerelated genes (HepG2) after aflatoxin B1 exposure. $\mathrm{PHH}$ gene expression responses are the most heterogeneous.

In conclusion, at base line level PHH are more similar to HepaRG than to HepG2, but for toxicogenomics applications both cell lines perform equally well in comparison to $\mathrm{PHH}$. 


\section{Introduction}

Toxicity testing necessary for assessing chemical and drug safety heavily relies on the use of rodent assays. Apart from being associated with obvious ethical drawbacks, these tests are also costly and timeconsuming [1]. In addition, data extrapolated from rodent assays to the human situation are criticized with regard to relevance, effectiveness and overall reliability. Therefore, efforts are put into developing in vitro human cell models for toxicity testing, including tests based on toxicogenomics approaches [2-5].

Since the liver is a key organ involved in the systemic toxicity response, many in vitro systems used for toxicity screening exploit hepatocytes $[6,7]$. Primary human hepatocytes $(\mathrm{PHH})$ can be isolated from liver tissue, cryopreserved and cultured in a sandwich-culture simulating its natural environment in the liver, without drastic functional changes in comparison to in vivo hepatocytes [8-10]. However, the life span of these cells is limited and so is their availability. Furthermore, large inter-individual differences in response to xenobiotic exposure are known to exist within the human population. Often, these interindividual differences are related to differences in expression/activity levels of xenobiotic metabolizing enzymes and transporters [11, 12].

In an attempt to overcome these $\mathrm{PHH}$-related drawbacks, several hepatocyte-based cell lines have been explored as a pragmatic alternative. The HepG2 cell line is derived from a human hepatocellular carcinoma, and it can be cultured unlimitedly under standard culture conditions. This has made HepG2 a very popular human cellular liver model in toxicity testing. Although this cell line shows many liver-specific functions, it also has some disadvantages [13], the most predominant being that in comparison to $\mathrm{PHH}$, HepG2 demonstrate lower levels of expression and activities of many xenobiotic metabolizing enzymes (Phase I and II biotransformation enzymes) and transporters [10, 1418]. These limitations might result in an erroneous toxicity assessment for compounds which require biotransformation. Nevertheless, HepG2s have been proven to be a useful model for toxicogenomics-based classification of compounds, also of those that require biotransformation $[4,14,15,19]$.

Another, newer, commercially available human liver carcinomaderived cell line is HepaRG. HepaRG cultures contain a mixture of hepatocyte-like and biliary-like epithelial cells. HepaRG cells, when cultured until confluence and with specific medium conditions, possess 
features corresponding to $\mathrm{PHH}$ [20]. In particular, HepaRG demonstrate expression of various Phase I enzyme activities at levels that are more comparable to $\mathrm{PHH}$ [20-25]. Therefore, HepaRG are said to represent a more suitable liver model than HepG2 for biological interpretations of effects of exposure to chemicals [26].

In a few studies, the full genome baseline gene expression profiles for the 3 above mentioned cell types have been compared [2628]. These show that the baseline gene expression profiles of HepaRG and HepG2 are distinct from those for $\mathrm{PHH}$, although the HepaRG profile is more $\mathrm{PHH}$-like.

However, in light of evaluating the comparability and applicability of these cell types for toxicogenomics studies, compoundinduced gene expression profiles are at least as important as baseline gene expression profiles. Several studies have shown that the gene expression responses from HepG2 and HepaRG in comparison to those from $\mathrm{PHH}$ are different after a xenobiotic challenge $[26,28]$. However, the number of $\mathrm{PHH}$ donors within these studies was limited $(n=3)$ and the Gerets study was performed with arrays containing a limited number of transcripts (1250 probes). Considering the large inter-individual variation in the human population, toxin-induced gene expression responses from many more $\mathrm{PHH}$ donors should be compared with the expression profiles of HepG2 and HepaRG to elucidate to what degree the responses of the cell lines fit within the responses as present in the human population.

Therefore, we compared both baseline and compound induced gene expression profiles from HepG2 and HepaRG with those in $\mathrm{PHH}$ from multiple ( $n=11-13)$ donors. For studying gene expression responses to compound exposure, we choose to use two mechanistically wellknown genotoxic carcinogens, that require metabolic activation, and that are often used in compound toxicity studies [29]. These compounds were aflatoxin B1 (AFB, a naturally occurring mycotoxin) and benzo( $\alpha$ ) pyrene (BaP, a product of incomplete combustion). Both genome wide expression changes and deregulations of pivotal signaling pathways (apoptosis, DNA-damage response, cell cycle and biotransformation) were investigated. 


\section{Materials and Methods}

\section{Cell culture and treatment}

\section{HepG2}

A HepG2 dataset exposed to $1 \mu \mathrm{M}$ AFB (CAS no. 1162-65-8, Sigma-Aldrich), $2 \mu \mathrm{M}$ BaP (CAS no. 50-32-8, Sigma-Aldrich) or $0.5 \%$ dimethyl sulfoxide (DMSO, CAS no. 67-68-5, Sigma-Aldrich) as a vehicle control for 24 hours was used. Dosing was based on the results from a MTT cytotoxicity assay with $80 \%$ viability following a $72 \mathrm{~h}$ treatment. Further details on culturing methods have been described before [26, 30].

\section{HepaRG}

A HepaRG dataset exposed to $2.5 \mu \mathrm{M} \mathrm{AFB}, 5 \mu \mathrm{M}$ BaP or $0.5 \%$ DMSO (vehicle control) for 24 hours was used. Dosing was based on the results from a MTT cytotoxicity assay with $80 \%$ viability following a $72 \mathrm{~h}$ treatment. Three independent experiments were conducted. Further details on culturing methods have been described before [26] .

Primary Human Hepatocytes

Cryopreserved $\mathrm{PHH}$ of 13 individuals were purchased from Life technologies (Gibco, see Table 1 for donor demographics and descriptive). PHH from donors of different, age, sex, ethnicity, body mass index and behavioral status concerning smoking, alcohol use and drug use were included, to create a diverse cell population enabling the evaluation of interindividual differences. Cells were cultured in 12well plates in a collagen sandwich, according to the supplier's protocol [31]. The following culture media were used: CHRM medium (Gibco) for thawing, William's medium E (WME)+Glutamax (Gibco) substituted with 10\% FCS (Gibco), 0.02\% penicillin/streptomycin (Gibco) and 0,1 $\mathrm{U} / \mathrm{ml}$ insulin (Invitrogen) for seeding/attaching and WME+Glutamax substituted with $0.02 \%$ Pen/Strep, $0.1 \mathrm{U} / \mathrm{ml}$ insulin and $0.02 \mathrm{mg} / \mathrm{ml}$ hydrocortisone (Sigma-Aldrich) for culturing/exposure. After thawing, viability of the cells was checked by a Trypan blue (CAS no. 72-57-1, Sigma-Aldrich) exclusion test as instructed in the supplier's protocol [31]. All viability scores after thawing were in accordance with those listed by the supplier. The hepatocytes were exposed to $1.25,2.5$ or $5 \mu \mathrm{M}$ AFB or $2.5,5$ or $10 \mu \mathrm{M} \mathrm{BaP}$ or $0.5 \%$ DMSO (vehicle control) in culture medium for 24 hours. Since PHH of the various donors are expected to respond differently with respect to cytotoxicity it was not possible to select one specific dose, therefore a dose range was included instead. To ensure comparability with the cell lines, we chose to include several doses for 
AFB/BaP which were in the same range as the doses used for HepG2 and HepaRG. Furthermore, the dose-range was based on similar studies with PHH [16, 32-34]. An incubation period of $24 \mathrm{~h}$ was chosen based on previous studies showing that: A) longer incubation periods ( $>12$ hours) were needed to induce clear gene expression changes by genotoxic compounds [35] and B) the time to ensure metabolism of genotoxic compounds was between 12-48 hours [26].

Table 1; Demographics of hepatocyte donors

\begin{tabular}{|l|l|l|l|l|l|l|l|l|}
\hline Donor & Race & Sex & $\begin{array}{l}\text { Age } \\
\text { (years) }\end{array}$ & BMI & Smoking & $\begin{array}{l}\text { Alcohol } \\
\text { use }\end{array}$ & $\begin{array}{l}\text { Drug } \\
\text { use }\end{array}$ & Cause of Death \\
\hline 1 & Caucasion & Female & 57 & 27 & No & No & No & Anoxia \\
\hline 2 & Unknown & Female & 28 & 26 & Yes & No & Yes & Anoxia \\
\hline 3 & Caucasion & Female & 59 & 32 & Yes & Yes & No & Cerebrovascular accident \\
\hline 4 & Caucasion & Male & 36 & 27.5 & Yes & Yes & Past & Head trauma \\
\hline 5 & Caucasion & Female & 1 & 18.3 & No & No & No & Intracerebral hemorrhage \\
\hline 6 & Caucasion & Female & 31 & 20 & No & Yes & No & Blunt trauma \\
\hline 7 & Caucasion & Female & 9 & 17.5 & No & No & No & Anoxia/head trauma \\
\hline 8 & Hispanic & Male & 0.25 & 15 & No & No & No & $\begin{array}{l}\text { Anoxia secondary to } \\
\text { cardiovascular }\end{array}$ \\
\hline 9 & Caucasion & Female & 57 & 20 & No & Yes & No & Intracerebral hemorrhage \\
\hline 10 & Indian & Female & 47 & 27 & No & Yes & No & Anoxia \\
\hline 11 & Caucasion & Female & 57 & 27.8 & No & No & No & Unknown \\
\hline 12 & Caucasion & Female & 36 & 30 & No & Yes & No & Unknown \\
\hline 13 & Caucasion & Female & 57 & 21 & Yes & No & No & Anoxia \\
\hline
\end{tabular}

\section{Transcriptomic sample preparation}

Total RNA isolation

Total RNA from all samples was isolated using $0.5 \mathrm{ml}$ QIAzol (QIAGEN) according to the manufacturer's protocol. RNA samples were purified using the miRNeasy Mini Kit (Qiagen) as instructed by the manufacturer. Subsequently, the RNA integrity was checked on a 2100 Bioanalyzer (Agilent) which resulted in an average RIN of 8.

cDNA preparation/hybridization

The preparation of cDNA/cRNA has been described in detail elsewhere [26]. All samples were hybridized according to the manufacturer's recommended procedures on Affymetrix Human Genome U133A plus 2 GeneChips [26]. After hybridization the GeneChips were washed and stained using a fluidics station (Affymetrix) and scanned with a GeneArray scanner (Affymetrix). PHH samples from 


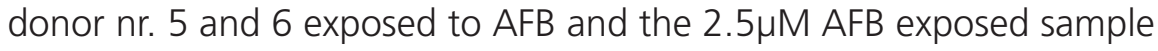
from donor nr. 1 did not perform well in the labeling procedure and were therefore excluded from further analyses.

\section{Data analyses}

Re-annotation, normalization and filtering

The pipeline from ArrayAnalysis.org (http://arrayanalysis.org) was used for re-annotation and normalization of the data. For short, data were re-annotated to EntrezGenelDs (based on a custom CDF from Brainarray [36]). The Robust Multi-array Average (RMA) method was used for data normalization, after which the data were log2transformed [37]. Furthermore, Present/Marginal/Absent (PMA) call tables were created. PMA calls were used to filter the data. For the HepG2 samples no more than 1 absent call was allowed within the triplicates for each treatment group. The same selection criteria were used for the HepaRG samples exposed to AFB and BaP. For the DMSO (control) samples 9 replicates were available and therefore no more than 3 absent calls were allowed within this group. Finally, PHH samples for which no replicates were available, were filtered as a group $(n=11-13$, one sample for each donor) concerning the vehicle control samples (DMSO), allowing no more than 4 absent calls per gene. For the other $\mathrm{PHH}$ treatment groups (AFB and BaP) data on 3 different doses for each compound were available per donor. A gene showing more than 1 absent call within these 3 samples was excluded in order to avoid inclusion of genes having an expression level too close to the detection limit of the arrays.

Baseline gene expression analyses

DMSO vehicle control samples were considered to demonstrate baseline gene expression levels. PMA filtering resulted in 3 lists of genes, one for each cell system (HepG2, HepaRG and PHH). In order to evaluate the similarities and differences between the cell systems, the union of these 3 lists was used

\section{Differentially expressed genes and union lists}

For PHH, HepG2 and HepaRG differentially expressed genes were calculated for either AFB or BaP (three independent biological replicates for each condition). For $\mathrm{PHH}$ this was done for each donor separately, thereby considering the samples from the three doses as replicates. First, log2-ratios between exposed cells and vehicle controls were calculated (for $\mathrm{PHH}$ this procedure was performed per donor). Differentially expressed genes were determined based on the following 
criteria: A) a log2-ratio $<-0.58$ or $>0.58$ (i.e., absolute fold change $>1.5$ ) for the average of each experimental group, B) a log2-ratio $<-0.26$ or $>0.26$ (i.e., absolute fold change $>1.2$ ) for at least 2 replicates and, C) the same direction of the log2-ratio for all the samples within an experimental group, as described previously [26]. After this, two union lists (one for transcriptomic responses to AFB and one for BaP) were created for the $\mathrm{PHH}$. Finally, a final union list was created per compound for the 3 cell systems.

Preselected gene sets

Four gene sets were collected to highlight gene expression profiles in the areas of: 1) apoptosis (Pathway:WP254, March 2011), 2) DNA-damage response (Pathway:WP707, March 2011), 3) cell cycle (Pathway:WP179, October 2011) and 4) biotransformation (phase I and II metabolism, receptors and modifiers, downloaded from pharmaADME.org).

Hierarchical clustering analysis (HCA) and principal component analysis (PCA)

HCA and PCA were applied to explore data similarities and differences in baseline gene expression levels between cell lines and in response to compound exposure, both in general and for the selected gene sets involved in apoptosis, DNA-damage, response to DNAdamage and biotransformation. For this, the HCA (Ward's method) and PCA module of Arraytrack were used [38, 39].

Pathway analysis and gene set enrichment

ToxProfiler, a freely available online analyses tool, was used to analyze the AFB and BaP data sets [40] to find deregulated pathways. All available Biocarta pathways, GO-processes, KEGG pathways and WikiPathways were investigated.

Toxprofiler uses the t-test (T-value) to score the difference between the mean expression level of predefined groups of genes and that of all other genes on the microarray. The T-value provides information on the intensity and direction of gene expression responses. From this T-value the E-value (a Bonferoni-corrected p-value) is calculated as a measure for statistical significance (E-value<0.05). In this study an E-value of 0.05 roughly corresponds to a T-value of + or -3.5 . Pathways significantly affected by a compound in at least 33\% of the PHH, HepG2 or HepaRG samples with a T-value in the same direction for at least $66 \%$ in $\mathrm{PHH}$ samples or $100 \%$ in HepG 2 or HepaRG samples, were collected. 
Furthermore, the predefined gene sets that are described above were uploaded into ToxProfiler as custom gene sets to assess the responses within these dedicated gene sets.

\section{Results}

\section{Gene selection}

The union list of the genes expressed at baseline that passed PMA-filtering within each cell system (HepG2, HepaRG or PHH) contains 12263 genes (Figure 1A).

The union of all differentially expressed genes for both exposure conditions results in 9531 and 4863 genes for AFB-exposed and BaP-exposed cell systems respectively (Figure $1 \mathrm{~B}+\mathrm{C}$ ). The $\mathrm{PHH}$ contribute most genes to the union list, due to the large interindividual variation.
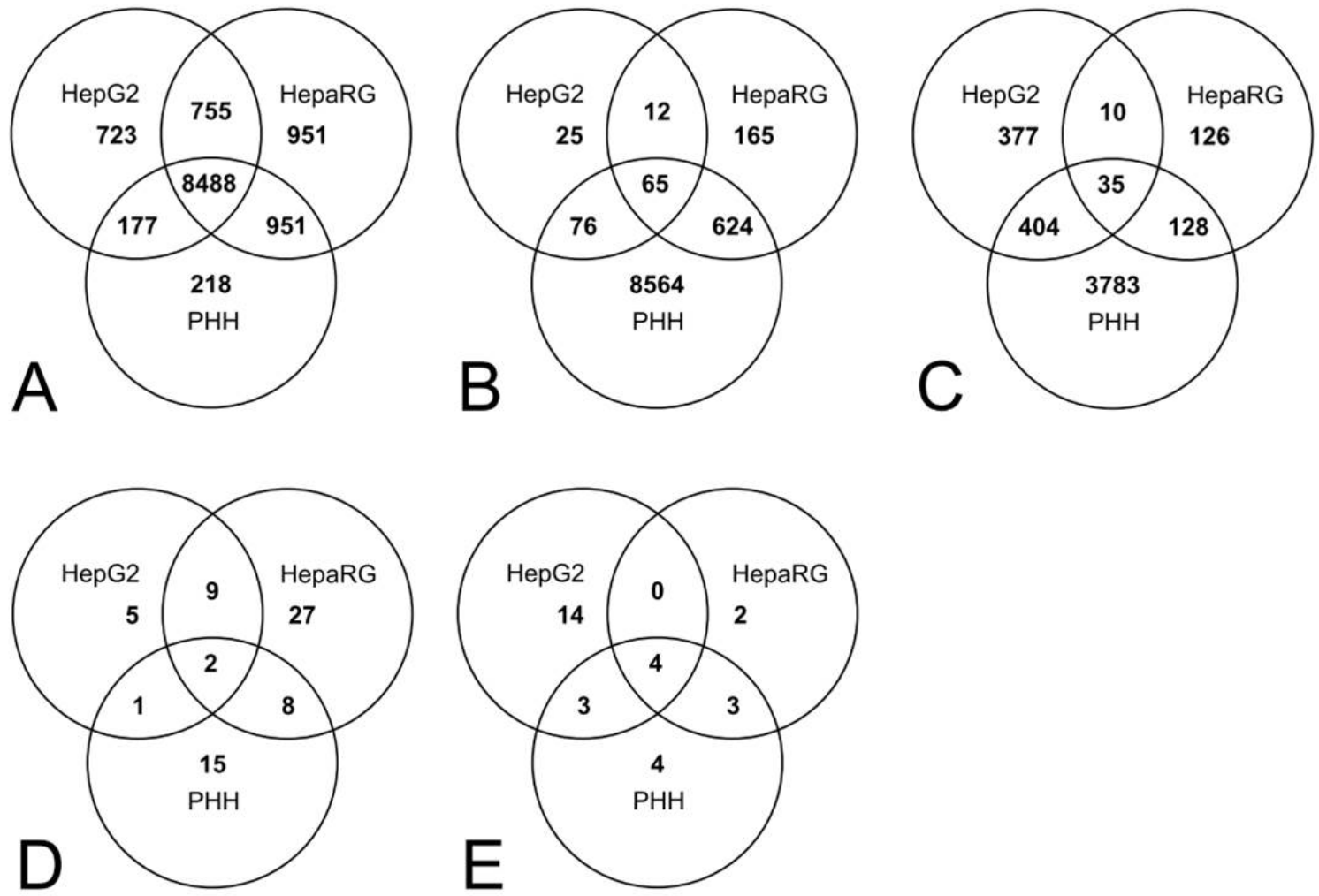

Figure 1; Overlapping genes/pathways

VENN diagrams compiling either gene-lists per cell model for A) all measured genes, and all differentially expressed genes for $B$ ) $A F B$ exposure and C) BaP exposure, or significantly affected pathways after D) $A F B$ exposure and E) BaP exposure. 


\section{Baseline gene expression profiles}

\section{PCA and HCA}

PCA and HCA on the full baseline gene expression profile, and on the preselected gene sets results in a complete separation of samples according to cell model (PCA: Figure 2A and supplementary Figure 1, HCA: supplementary Figure 4, baseline). Although still very different, HepaRG baseline gene expression levels are more common to $\mathrm{PHH}$ in the gene sets preselected for apoptosis and biotransformation. A visualization of the expression of genes within the pre-selected gene sets can be found in supplementary Figure 5 .

In order to avoid overt a-specific responses due to cell death, toxicogenomic studies are normally conducted at doses that cause limited cytotoxicity. The doses for the HepG2 and HepaRG studies were based on an MTT-test [41], with an $80 \%$ viability [26]. Since PHH were expected to show inter-individual differences in cytotoxicity, we investigated the same 3 doses in PHH from all donors, and chose dose levels in the same range as for the cell lines. Several studies on dose and time effects of compounds within the cell systems used in the current study have shown that dose-dependent differences are relatively small [42-44].
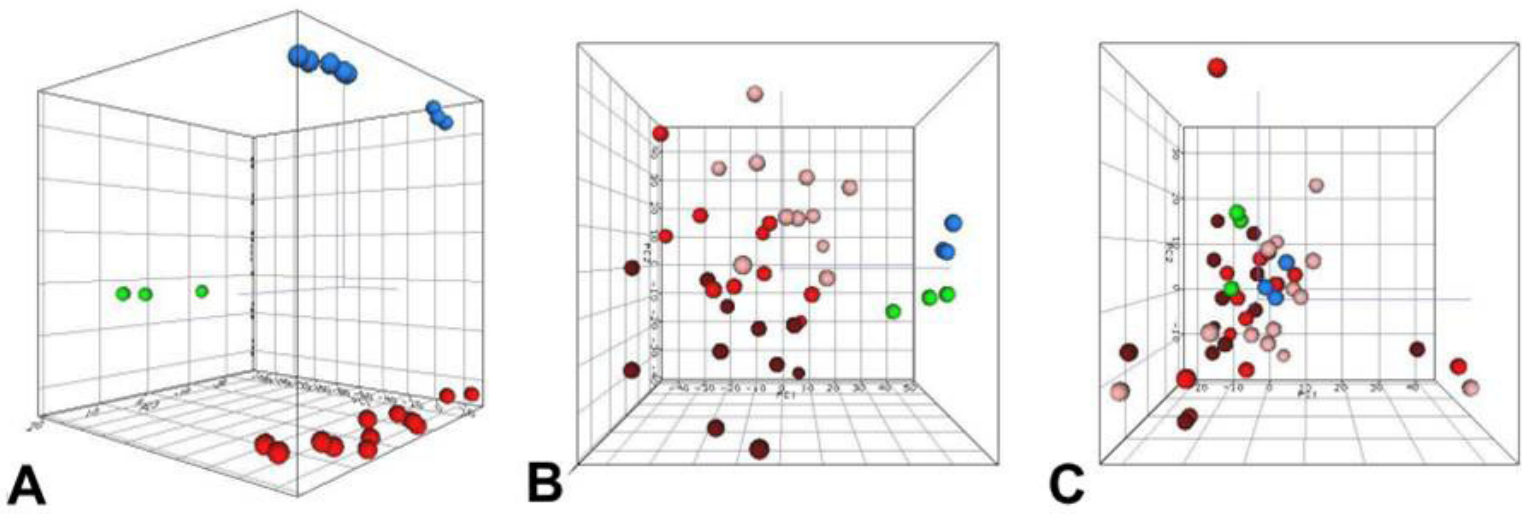

Figure 2; Separation of cell models by PCA

Principal component analyses based on all genes for A) un-exposed control cells (HepG2 (green), HepaRG (blue) and PHH (red)), B) AFB exposed cells and C) BaP exposed cells (HepG2 (green), HepaRG (blue) and PHH (pink lowest dose, red medium dose and dark red highest dose) 
AFB-induced gene expression profiles

HCA, PCA and gene set enrichment analysis was performed using the log2-ratio data from the AFB union gene-list and the preselected gene sets.

PCA and HCA

The gene expression profiles in response to AFB are again separated based on cell type using PCA (Figure 2B and supplementary Figure 2) and HCA (supplementary Figure 4, Aflatoxin B1). While the variation in transcriptomic response between the HepG2 or HepaRG samples is relatively small, large variation exists among $\mathrm{PHH}$ samples. Especially samples from donor 11 have a gene expression profile that is different from all other donors in the gene set preselected for biotransformation. The most obvious differences for donor 11 are observed with regard to CYP1A1, CYP1A2 and CYP1B1 expression (data not shown). However, for all gene sets the gene expression responses to AFB for HepG2 and HepaRG are more similar to one another than they are to the responses of $\mathrm{PHH}$.

Pathway analysis and gene set enrichment

In total 19 BioCarta pathways, 122 GO-processes, 61 KEGG pathways and 34 WikiPathways are found to be significantly altered as a consequence of AFB exposure in at least one of the cell models. Pathways significantly affected in a similar direction (based on T-value) in $\mathrm{PHH}$, HepG2 and HepaRG, are depicted in a Venn-diagram (Figure 1D). The AFB induced response in the 3 cell systems includes pathways related to DNA-damage, biotransformation and receptor mediated processes (including toxicity related receptor responses). Two pathways are regulated significantly and in the same direction in all 3 cell models; 'peroxisome GO process' and 'p53 signaling pathway KEGG pathway' (Figure 1D and supplementary Table 1A). A total of 10 pathways are significantly affected in the same direction in HepaRG and PHH, while in HepG2 and PHH only 3 pathways are affected similarly (Figure 1D and supplementary Table 1A). All 3 cell models show pathways that are model specific e.g. are significantly induced in just one of the cell systems: 15 in PHH, 27 in HepaRG and 5 in HepG2 (supplementary Table 1A).

Also gene set enrichment analyses for the pre-selected gene sets (Figure 3) indicates that HepG2 and HepaRG respond different to AFB exposure than $\mathrm{PHH}$. T-values for the cell lines are sometimes opposite in direction to those from PHH (see sets for apoptosis (HepG2 and HepaRG) and cell cycle (HepG2)), indicating oppositely regulated 
gene expression responses. Moreover, the T-values for the cell lines often exceed the T-values from the PHH (sets for DNA-damage and cell cycle (HepG2 and HepaRG) and biotransformation (HepaRG)). Genes driving the responses for the pre-selected gene sets can be found in supplementary Figure 5.
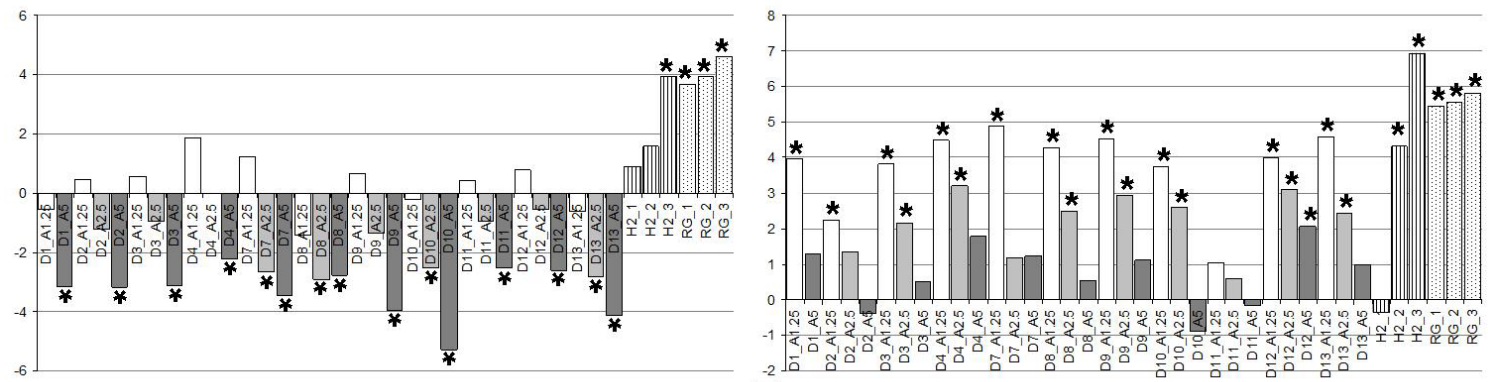

A

B
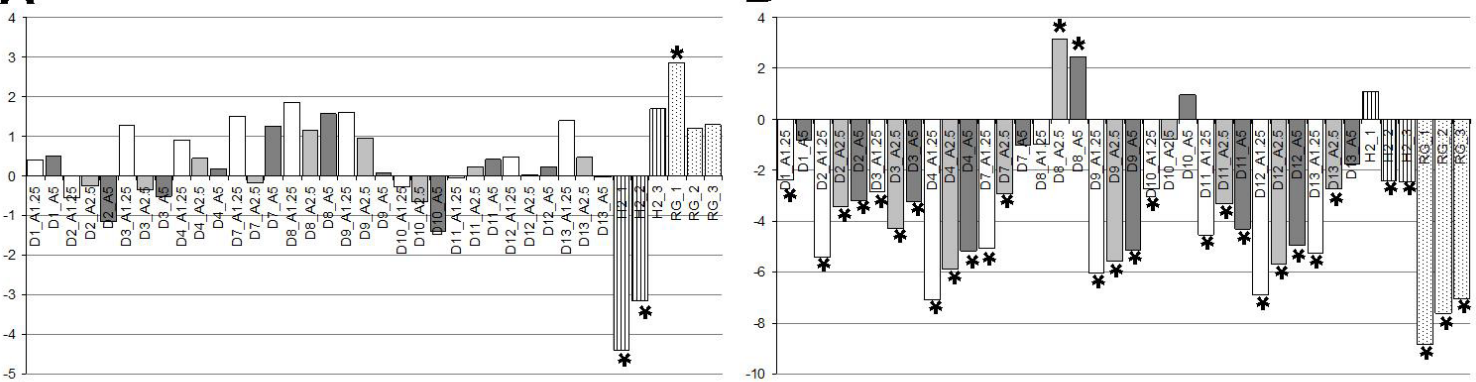

D

Figure 3; Gene set enrichment for several pre-defined gene sets and for various cell models after AFB exposure

Gene set enrichment after exposure to AFB for HepG2 (H2, striped), HepaRG (RG, dotted) and PHH (D\#, white $1.25 \mu \mathrm{M}$, mid gray $2.5 \mu \mathrm{M}$, dark gray $5 \mu \mathrm{M}$ ) for gene sets selected for $A$ ) apoptosis, $B$ )

DNA-damage, C) cell cycle and D) biotransformation. Y-axis represents the T-value, * E-value<0.05.

More information of the T/E-values can be found in the methods section.

\section{BaP induced gene expression profiles}

A PCA, HCA and gene set enrichment analysis was performed using the log2-ratio data from the BaP union gene-list and the preselected gene sets.

PCA and HCA analyses

The BaP-induced gene expression profiles for HepG2 and HepaRG are similar to those from PHH (PCA: Figure $2 \mathrm{C}$ and supplementary Figure 3, HCA: supplementary Figure 4, Benzo( $\alpha)$ pyrene). This is true for the full gene set and the pre-selected gene sets. In the gene set preselected for biotransformation, HepG2 cells show a gene 
expression profile that is different from HepaRG or PHH. However, in general inter-individual variation between $\mathrm{PHH}$ samples exceeds the variation between the different cell types. The gene expression profile from donor 11 is different from all other PHH, HepG2 or HepaRG gene expression profiles.

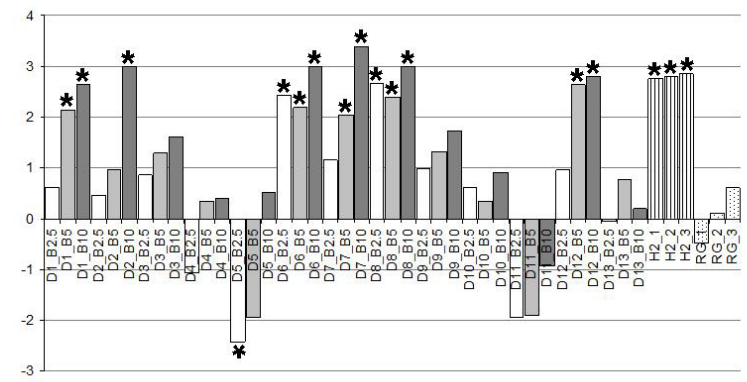

A

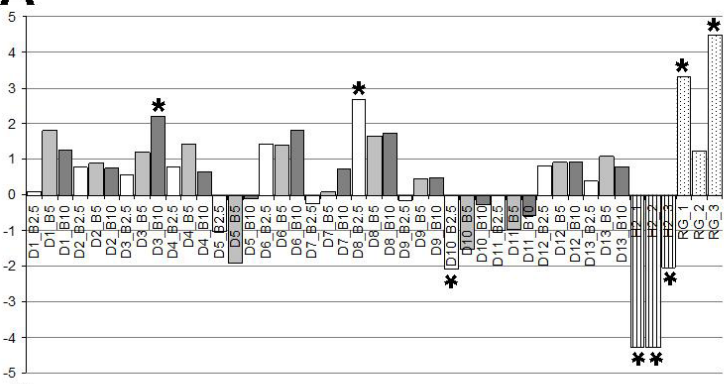

C

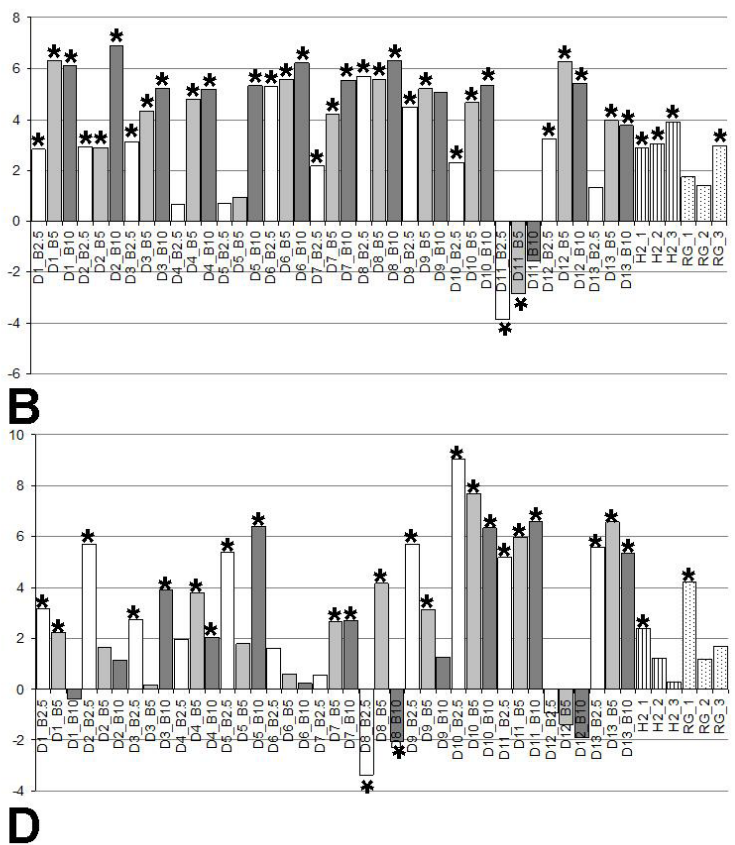

D

Figure 4; Gene set enrichment for several pre-defined gene sets and for the various cell models after BaP exposure

Gene set enrichment after exposure to BaP for HepG2 (H2, striped), HepaRG (RG, dotted) and PHH (D\#, white $2.5 \mu \mathrm{M}$, mid gray $5 \mu \mathrm{M}$, dark gray $10 \mu \mathrm{M}$ ) for gene sets selected for $A$ ) apoptosis, B) DNAdamage, C) cell cycle and D) biotransformation. Y-axis represents the T-value, * E-value<0.05. More information of the T/E-values can be found in the methods section.

Gene set enrichment

In total 6 BioCarta pathways, 50 GO-processes, 37 KEGG pathways and 25 WikiPathways are found to be significantly altered as a consequence of BaP exposure in at least one of the samples. Pathways significantly affected in a similar direction (based on T-value) in $\mathrm{PHH}$, HepG2 and HepaRG, are depicted in a Venn-diagram (Figure 1E). The overlap in this Venn-diagram is summarized in supplementary Table 1B. The BaP induced response in the 3 cell systems includes pathways related to DNA-damage and biotransformation related processes. Four pathways are regulated significantly and in the same direction in all 3 cell models; 'oxygen binding', 'aromatase activity', 'heme binding' and 'metabolism of xenobiotics by cytochrome P450' (Figure 1E and 
supplementary Table 1B). All 3 cell models show pathways that are model specific e.g. are significantly induced in just one of the cell systems; 4 in PHH, 2 in HepaRG and 14 in HepG2 (supplementary Table 1B).

In addition, a gene set enrichment analysis of the pre-selected pathways indicates that the gene expression response to $\mathrm{BaP}$ in HepG2 and HepaRG in general is comparable to the responses in PHH (Figure 4). With the exception of the T-values in the apoptosis gene set (HepG2 only) and cell cycle gene set (HepG2 and HepaRG), T-values for the cell lines are largely similar to the T-values from $\mathrm{PHH}$. The genes driving the responses for the pre-selected gene sets can be found in supplementary Figure 5.

\section{Discussion}

This study aims at elucidating the (dis)similarities in baseline and compound-induced gene expression profiles of HepG2, HepaRG and $\mathrm{PHH}$, thereby especially taking into account the inter-individual variability in $\mathrm{PHH}$. This is of crucial importance in light of the applicability of these cell types for toxicogenomics studies and for assessing the variation in the human population. To do so, baseline and AFB- or BaP-induced gene expression profiles were compared between the 3 cell models. In order to assess the expected inter-individual variability within the human population, $\mathrm{PHH}$ from multiple $(n=11-13)$ donors were investigated. Pathway analysis was used to gain insight into the underlying biology of differentially expressed genes in each of the cell system. In addition, preselected gene sets dedicated to apoptosis, DNA-damage, cell cycle and biotransformation were investigated in-depth because of their crucial role in all cellular systems, especially with respect to effects caused by genotoxic carcinogens [45], and their expected involvement in the metabolism of the compounds used within this study.

\section{Baseline gene expression profiles}

PCA on the full gene list separates the 3 cell models based on cell type (Figure 2A). Baseline gene expression patterns from the cell models thus differ profoundly from each other. Other studies comparing baseline gene expression profiles from PHH, HepaRG and HepG2 are in agreement with this finding [26-28]. This separation of cell types after PCA is also consistent for gene sets selected for apoptosis, DNAdamage, cell cycle and biotransformation (supplementary Figure 1). 
Both PCA and HCA indicate that PHH are more similar to HepaRG than to HepG2. This is true for the full gene list, and for the pre-defined gen sets for apoptosis and biotransformation.

For apoptosis related genes, it has been shown before that their expression in $\mathrm{PHH}$ is more similar to the expression in HepaRG than in HepG2 [26].

For other gene sets, such as on DNA-damage response and cell cycle, the baseline gene expression profiles were more similar between both cell lines, emphasizing that there are also many differences between PHH, HepaRG and HepG2.

Interindividual variation between baseline gene expression profiles from different hepatocyte donors have been shown before [28, 46] and are confirmed by our study. Factors related to interindividual variation include age, sex, body mass index, cause of death and ethnicity. For the PHH population used within this study, only age is a factor that explains some of the clustering of the samples at baseline gene expression levels (4th component in PCA analysis, explains $6.7 \%$ of variation, data not shown).In the HCA-plot using the full list of genes at baseline expression level (supplementary Figure 4), donors 5 and 8 are separated from the other donors, which can be related to the young age of these donors ( 1 year and 3 months old respectively, see also Table 1). Infants are known to have deviating gene expression levels for many genes, especially those related to biotransformation [47]. Age effects in the gene expression levels of $\mathrm{PHH}$ are persistent, also after compound exposure as will be discussed in further detail below. None of the other factors mentioned above could explain clustering of the $\mathrm{PHH}$ samples.

The interindividual variation between $\mathrm{PHH}$ is considered problematic when using these cells for human hazard identification. However, despite this variation, the baseline gene expression profiles of all PHH are more similar to each other than to those of HepG2 or HepaRG. Within the HepaRG system a variation between samples is seen that exceeds the interindividual variation between $\mathrm{PHH}$ samples. Also in the study performed by Rogue et al. the variation in the PPAR $\alpha$ expression level is larger between HepaRG replicates than between PHH from different donors [48]. This variation might be due to variation of cell types in HepaRG cultures. The fraction of hepatocyte-like cells in HepaRG cultures varies from $45-90 \%$ between batches and passages [49]. 
We therefore conclude that based on baseline gene expression profiles HepG2, HepaRG and PHH are very different from each other and thus neither of the cell line models is a complete alternative to $\mathrm{PHH}$.

However, for human risk assessment using toxicogenomic applications, similarities of gene expression responses following toxic insults may be more relevant for comparing the effectiveness of in vitro cell models. We explored this for two chemical carcinogens, i.e. AFB and $\mathrm{BaP}$.

\section{AFB-induced gene expression profiles}

After AFB exposure, the different cell types can again be separated by both PCA and HCA based on the full gene set and all preselected gene sets (Figure 2B and supplementary Figures 2+4). This indicates that differences between the cell models still exist after compound exposure. However, in comparison to the baseline gene expression profiles, AFB-induced gene expression profiles from HepG2 and HepaRG cells are now more similar to $\mathrm{PHH}$.

Two processes, 'peroxisome GO process' and 'p53 signaling pathway KEGG pathway', are regulated significantly and in the same direction in all 3 cell models. While peroxisome related processes are linked to the metabolism of AFB [50], p53 related processes can be assigned to the DNA-damaging properties of AFB [51].

In contrast to what is observed at baseline gene expression levels, AFB-induced gene expression profiles do not show either of the 2 cell lines to be more similar to PHH (Figure 1B, supplementary Figure 2 and supplementary Figure 4, Aflatoxin B1). HepaRG cells show a larger number of genes/pathways regulated after AFB exposure in comparison to HepG2. Many of these genes/pathways are specific to the response of the HepaRG system and are involved in carbohydrate metabolism related processes which are known to be affected by AFB [52]. The $\mathrm{PHH}$ show a response to $\mathrm{AFB}$ that involves more signaling and receptor related processes. Indeed, AFB has recently been suggested to activate the aryl hydrocarbon receptor (AhR), constitutive androstane receptor (CAR) and pregnane $\mathrm{X}$ receptor (PXR) in $\mathrm{PHH}$ [53]. Baseline expression levels of these receptors in differentiated HepaRG and cryopreserved $\mathrm{PHH}$ are shown to be similar [54]. However, in response to compounds receptors seem differentially regulated in HepaRG and PHH [55]. The current study suggests that dissimilarities in compound induced gene expression levels related to cellular receptors, might at least in part 
explain the differences seen in the responses of PHH and HepaRG to AFB exposure. However, further studies will have to be executed to confirm these results.

Also gene set enrichment analyses for the pre-selected gene sets (Figure 3) indicate that HepG2 and HepaRG respond different to AFB exposure than $\mathrm{PHH}$.

T-values of the cell lines for apoptosis in HepG2 and HepaRG and for cell cycle in HepG2 are opposite to those in $\mathrm{PHH}$, indicating oppositely regulated gene expression responses. Apoptosis-related processes, which are often affected in carcinoma cells [56], were expected to respond differently to AFB exposure in both cell lines in comparison to in $\mathrm{PHH}$ [57]. In addition slight differences in endogenous metabolism between the cell systems can cause differences in the apoptosis response; however we have no data supporting this statement.

No, or moderate changes in cell cycle related genes in $\mathrm{PHH}$ and HepaRG were to be expected, since both models are nonproliferating. Cell cycle related genes in HepG2 cells are predominantly down-regulated after AFB exposure. AFB is known to impair cell cycle progression in HepG2, thus decreasing proliferation in these cells [58].

In addition, T-values for the cell lines exceed the T-values from the PHH in the sets for DNA-damage and cell cycle (HepG2 and HepaRG) and in the biotransformation set (HepaRG). Although AFB induced gene expression changes in DNA-damage related responses are more explicit in HepG2 and HepaRG, the compound induced gene expression profile in $\mathrm{PHH}$ does involve a broader set of genes. As described above, differences in cell cycle related processes were expected for HepG2, as it is the only differentiating cell system. The deviating T-value for HepaRG can be explained by the aberrant expression of several cell cycle related genes that have also been linked to tumor growth. The aberrant response of these genes could very well be a consequence of the carcinoma derived background of HepaRG.

As already discussed above, the deviating T-value for the biotransformation related response in HepaRG can be associated to cell model-specific expression of genes involved in carbohydrate metabolism. PHH samples show variation due to both inter-individual differences and a dose-related AFB effect. The effects of inter-individual variation surpass the effects of dose. Interestingly, the AFB-induced 
gene expression profile for biotransformation related genes in HepaRG and HepG2 is most similar to the expression pattern from donor 8, the very young $\mathrm{PHH}$ donor. The gene expression response to AFB exposure in both cell lines and the PHH from donor 8 includes fewer genes in comparison to all other $\mathrm{PHH}$. Thus, the cell lines seem more restricted in their ability to respond to AFB exposure in comparison to $\mathrm{PHH}$.

Among all other PHH donors, donor 11 appears to have a gene expression profile that is aberrant from all other donors, HepG2 and HepaRG. The most striking differential expression of genes in donor 11 is seen in the biotransformation gene set, especially in CYP1A1, CYP1A2 and CYP1B1 (data not shown). CYP1A1, CYP1A2 and CYP1B1, are known to be involved in the metabolism of $A F B$, and are all regulated by $\operatorname{AhR}[53,59-61]$. These genes are strongly down-regulated in donor 11 , but mostly up-regulated in the other donors. However the absolute level of expression of the CYPs is similar between all donors after AFB exposure (data not shown). Donor 11 thus appears to have a very high baseline expression level of the above mentioned CYPs, possibly due to a constitutive activation of AhR.

Nevertheless, despite clear inter-individual variation in the AFB induced gene expression profiles in $\mathrm{PHH}$, their responses still resemble each other more than that they resemble those for HepG2 or HepRG.

All together, we conclude that the AFB-induced gene expression patterns of the cell lines are more similar to one another than to those of $\mathrm{PHH}$, despite the large inter-individual variation for the $\mathrm{PHH}$. At the level of affected pathways, PHH show more similarities with HepaRG than with HepG2.

\section{BaP-induced gene expression profiles}

The BaP-induced gene expression profiles from the 3 cell types are indistinguishable from each another, which contrasts from the gene expression profiles found at baseline levels or after induction wit AFB (Figure 2C + supplementary Figure 3+4).

'Oxygen binding', 'aromatase activity', 'heme binding' and

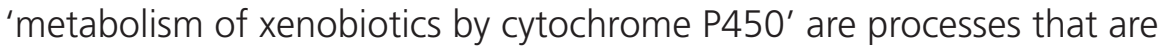
regulated significantly and in the same direction in all 3 cell models. All of these processes are linked to the metabolism of BaP by cytochrome P450 [26]. 
Since the response to $\mathrm{BaP}$ is similar for all 3 cell models, only a few cell model specific responses exist. PHH show a broader set of responding genes related to DNA-damage, which is in line with the response from these cells seen after AFB exposure. More pronounced is the higher amount of responding genes/pathways related to cell cycle in HepG2 cells. Enrichment analyses on the gene set pre-selected for cell cycle related genes also shows that HepG2 have a deviating gene expression profile in comparison to $\mathrm{PHH}$. A known effect of $\mathrm{BaP}$ is the induction of cell cycle arrest at the $\mathrm{G} 1$-phase $[26,43]$. In contrary to $\mathrm{PHH}, \mathrm{HepG} 2$ are proliferating cells. Consequently a BaP induced cell cycle arrest in HepG2 could explain the above described response. As after AFB exposure the T-value for cell cycle related genes in HepaRG is aberrant from $\mathrm{PHH}$. As discussed before, aberrant expression of tumor related genes causes this effect that might be inherent to the carcinoma derived background of HepaRG.

The largest variation in gene expression profiles following $\mathrm{BaP}$ exposure are observed within the PHH samples, again mainly due to inter-individual differences. The different dose levels of BaP do cause a lot of variance within the $\mathrm{PHH}$ system. A lack of dose response after $\mathrm{BaP}$ exposure has been described before in HepG2 cells [42]. Gene expression responses from donor 11 again are aberrant from all other donors (supplementary Figure 4). Like after AFB exposure, regulation of CYP1A1, CYP1A2 and CYP1B1 expression levels are different in this donor. Expression of these genes is not induced by BaP in cells from donor 11 (rather reduced in expression), but is strongly up-regulated in cells from other donors, HepG2 and HepaRG. BaP is a well-known ligand activator for AhR and normally induces the transcription of these CYPs, which again indicates that in donor $11 \mathrm{AhR}$ is malfunctioning.

All together, we conclude that the BaP-induced gene expression patterns of the carcinoma derived cell lines differ from each other, but are not distinguishable from those of $\mathrm{PHH}$. At the level of affected pathways, all 3 cell models show model-specific regulation of pathways, and neither of the carcinoma derived cell lines is more similar to $\mathrm{PHH}$.

In addition to the analyses described above, the overall effects of AFB and BaP versus the control values were also plotted in a PCA. The direction of responses appears similar for both compounds in all cell types (data not shown). The similarity in direction of response after AFB and $\mathrm{BaP}$ exposure can be explained by the shared genotoxic properties and AhR driven metabolite activation of these compounds.Although similar, the compound specific responses are different enough for the 
cell systems to be distinguished, which is an indicator for robustness of the cell systems. An example of a more subtle difference between the responses to $\mathrm{AFB}$ and $\mathrm{BaP}$ is the response in nuclear receptors. Both compounds induce a response from nuclear receptors, but BaP gives a much more pronounced response. Possibly this can be explained by the difference in receptors involved in compound metabolism; BaP mainly activates AhR receptors while the AFB-induced response involves AhR, PXR and CAR receptors.

As far as we know, this is the first study comparing both baseline and compound-induced full-genome gene expression patterns for HepG2 and HepaRG, with PHH. Also, no other study is known to us comparing gene expression patterns from as many individuals as in this study.

The gene expression responses of $\mathrm{PHH}$ in the area of biotransformation, DNA-damage, apoptosis and cell cycle are more diverse than the responses from HepG2 and HepaRG. Therefore, PHH should still be considered as the prime model for collecting molecular/ mechanistic information in the evaluation of genotoxicity responses. This is in line with the findings of Gerets et al. who concluded that $\mathrm{PHH}$ outperform HepG2 and HepaRG for the prediction of drug induced hepatotoxicity in humans [28].

Interindividual variability could possibly be overcome at least partially by the use of pooled $\mathrm{PHH}$, i.e. the use of $\mathrm{PHH}$ from multiple donors in one single culture. The commercial availability of cryopreserved pooled $\mathrm{PHH}$ is increasing steadily, which enables to perform multiple experiments on PHH of the same set of donors.

In summary, our study shows that (A) at baseline gene expression level the 3 cell models differ profoundly from each other, while (B) after compound exposure gene expression changes in the cell lines are more similar to those of $\mathrm{PHH}$. Thus, based on the current study, no conclusion can be made about which cell line gives in toxicogenomics studies the best comparability with $\mathrm{PHH}$. In a few cases, however, PHH are more similar to HepaRG than to HepG2, i.e. for baseline gene expression and AFB-affected pathways. Further studies exploring a broader range of compounds will also have to point out whether the conclusions from this study can be generalized for other (groups of) compounds. 


\section{Supplementary Tables / Figures}

Supplementary Table 1 and Supplementary Figure 1-5: Available online: http://www.sciencedirect.com/science/article/pii/ S0887233313001884

\section{Acknowledgments}

This work was supported by CarcinoGENOMICS, a part of the 6th framework project of the European Union aimed at developing in vitro methods for assessing the carcinogenic potential of compounds, as an alternative to current rodent bioassays for genotoxicity and carcinogenicity. Furthermore, we would like to thank C. Magkoufopoulou and D. Jennen for providing us with all the information regarding the HepG2 data.

\section{Conflict of interest statement}

C. Chesné declares to have a conflict of interest because of directing in BIOPREDIC promoting the HepaRG cells; however $C$. Chesné was not involved in any data analyses or the interpretation of the results from this study. Nor did BIOPREDIC provide any funding for this study. All other authors declare that there are no conflicts of interest. 


\section{References}

1. Kroeger, M., How omics technologies can contribute to the '3R' principles by introducing new strategies in animal testing. Trends Biotechnol, 2006. 24(8): p. 343-6.

2. Dambach, D.M., B.A. Andrews, and F. Moulin, New technologies and screening strategies for hepatotoxicity: use of in vitro models. Toxicol Pathol, 2005. 33(1): p. 17-26.

3. Harrill, A.H. and I. Rusyn, Systems biology and functional genomics approaches for the identification of cellular responses to drug toxicity. Expert Opin Drug Metab Toxicol, 2008. 4(11): p. 1379-89.

4. van Delft, J.H., et al., Discrimination of genotoxic from non-genotoxic carcinogens by gene expression profiling. Carcinogenesis, 2004. 25(7): p. $1265-76$.

5. Magkoufopoulou, C., et al., A transcriptomics-based in vitro assay for predicting chemical genotoxicity in vivo. Carcinogenesis, 2012. 33(7): p. 1421-9.

6. DeLeve, L.D. and N. Kaplowitz, Mechanisms of drug-induced liver disease. Gastroenterol Clin North Am, 1995. 24(4): p. 787-810.

7. Gebhardt, R., Metabolic zonation of the liver: regulation and implications for liver function. Pharmacol Ther, 1992. 53(3): p. 275-354.

8. Guillouzo, A., Liver cell models in in vitro toxicology. Environ Health Perspect, 1998. 106 Suppl 2: p. 511-32.

9. Gomez-Lechon, M.J., et al., Human hepatocytes in primary culture: the choice to investigate drug metabolism in man. Curr Drug Metab, 2004. 5(5): p. 443-62.

10. Olsavsky, K.M., et al., Gene expression profiling and differentiation assessment in primary human hepatocyte cultures, established hepatoma cell lines, and human liver tissues. Toxicol Appl Pharmacol, 2007. 222(1): p. 42-56.

11. Ma, M.K., M.H. Woo, and H.L. McLeod, Genetic basis of drug metabolism. Am J Health Syst Pharm, 2002. 59(21): p. 2061-9.

12. Zhou, S.F., J.P. Liu, and B. Chowbay, Polymorphism of human cytochrome P450 enzymes and its clinical impact. Drug Metab Rev, 2009. 41(2): p. 89-295.

13. Liguori, M.J., E.A. Blomme, and J.F. Waring, Trovafloxacin-induced gene expression changes in liver-derived in vitro systems: comparison of primary human hepatocytes to HepG2 cells. Drug Metab Dispos, 2008. 36(2): p. 223-33.

14. Westerink, W.M. and W.G. Schoonen, Cytochrome P450 enzyme levels in HepG2 cells and cryopreserved primary human hepatocytes and their induction in HepG2 cells. Toxicol In Vitro, 2007. 21(8): p. 1581-91.

15. Westerink, W.M. and W.G. Schoonen, Phase II enzyme levels in HepG2 cells and cryopreserved primary human hepatocytes and their induction in HepG2 cells. Toxicol In Vitro, 2007. 21(8): p. 1592-602.

16. Wilkening, S., F. Stahl, and A. Bader, Comparison of primary human hepatocytes and hepatoma cell line Hepg2 with regard to their biotransformation properties. Drug metabolism and disposition: the biological fate of chemicals, 2003. 31(8): p. 1035-42.

17. Guo, L., et al., Similarities and differences in the expression of drugmetabolizing enzymes between human hepatic cell lines and primary human hepatocytes. Drug Metab Dispos, 2011. 39(3): p. 528-38.

18. Jennen, D.G., et al., Biotransformation pathway maps in WikiPathways enable direct visualization of drug metabolism related expression changes. Drug 
Discov Today, 2010. 15(19-20): p. 851-8.

19. Song, M.K., et al., Identification of molecular signatures predicting the carcinogenicity of polycyclic aromatic hydrocarbons (PAHs). Toxicol Lett, 2012. 212(1): p. 18-28.

20. Aninat, C., et al., Expression of cytochromes P450, conjugating enzymes and nuclear receptors in human hepatoma HepaRG cells. Drug Metab Dispos, 2006. 34(1): p. 75-83.

21. Turpeinen, M., et al., Functional expression, inhibition and induction of CYP enzymes in HepaRG cells. Toxicol In Vitro, 2009. 23(4): p. 748-53.

22. Lambert, C.B., et al., Reproducible chemical-induced changes in gene expression profiles in human hepatoma HepaRG cells under various experimental conditions. Toxicol In Vitro, 2009. 23(3): p. 466-75.

23. Kanebratt, K.P. and T.B. Andersson, Evaluation of HepaRG cells as an in vitro model for human drug metabolism studies. Drug Metab Dispos, 2008. 36(7): p. 1444-52.

24. Kanebratt, K.P. and T.B. Andersson, HepaRG cells as an in vitro model for evaluation of cytochrome P450 induction in humans. Drug Metab Dispos, 2008. 36(1): p. 137-45.

25. Guillouzo, A., et al., The human hepatoma HepaRG cells: a highly differentiated model for studies of liver metabolism and toxicity of xenobiotics. Chem Biol Interact, 2007. 168(1): p. 66-73.

26. Jennen, D.G., et al., Comparison of HepG2 and HepaRG by whole-genome gene expression analysis for the purpose of chemical hazard identification. Toxicol Sci, 2010. 115(1): p. 66-79.

27. Hart, S.N., et al., A comparison of whole genome gene expression profiles of HepaRG cells and HepG2 cells to primary human hepatocytes and human liver tissues. Drug Metab Dispos, 2010. 38(6): p. 988-94.

28. Gerets, H.H., et al., Characterization of primary human hepatocytes, HepG2 cells, and HepaRG cells at the mRNA level and CYP activity in response to inducers and their predictivity for the detection of human hepatotoxins. Cell Biol Toxicol, 2012. 28(2): p. 69-87.

29. Zhou, S.F., et al., Structure, function, regulation and polymorphism and the clinical significance of human cytochrome P450 1A2. Drug Metab Rev, 2010. 42(2): p. 268-354.

30. Magkoufopoulou, C., et al., Comparison of phenotypic and transcriptomic effects of false-positive genotoxins, true genotoxins and non-genotoxins using HepG2 cells. Mutagenesis, 2011. 26(5): p. 593-604.

31. Invitrogen, No Standard Like a Gold Standard - InvitrogenTM Hepatic Biology Products. Invitrogen product brochure, 2009: p. 11-15.

32. Plazar, J., et al., Detection of xenobiotic-induced DNA damage by the comet assay applied to human and rat precision-cut liver slices. Toxicology in vitro an international journal published in association with BIBRA, 2007. 21(6): p. 1134-1142.

33. Li, A.P., Metabolism Comparative Cytotoxicity Assay (MCCA) and Cytotoxic Metabolic Pathway Identification Assay (CMPIA) with cryopreserved human hepatocytes for the evaluation of metabolism-based cytotoxicity in vitro: proofof-concept study with aflatoxin B1. Chem Biol Interact, 2009. 179(1): p. 4-8.

34. Langouet, S., et al., Metabolism of aflatoxin B1 by human hepatocytes in primary culture. Adv Exp Med Biol, 1996. 387: p. 439-42.

35. Mathijs, K., et al., Discrimination for genotoxic and nongenotoxic carcinogens by gene expression profiling in primary mouse hepatocytes improves with exposure time. Toxicol Sci, 2009. 112(2): p. 374-84. 
36. University of Michigan. Brainarray custom CDF. Version 13; Available from: http://brainarray.mbni.med.umich.edu/Brainarray/Database/CustomCDF/ cdfreadme.htm.

37. Irizarry, R.A., et al., Exploration, normalization, and summaries of high density oligonucleotide array probe level data. Biostatistics, 2003. 4(2): p. 249-64.

38. Tong, W., et al., ArrayTrack--supporting toxicogenomic research at the U.S. Food and Drug Administration National Center for Toxicological Research. Environ Health Perspect, 2003. 111(15): p. 1819-26.

39. Tong, W., et al., Development of public toxicogenomics software for microarray data management and analysis. Mutat Res, 2004. 549(1-2): p. 241-53.

40. Boorsma, A., et al., T-profiler: scoring the activity of predefined groups of genes using gene expression data. Nucleic Acids Res, 2005. 33(Web Server issue): p. W592-5.

41. Cole, S.P., Rapid chemosensitivity testing of human lung tumor cells using the MTT assay. Cancer Chemother Pharmacol, 1986. 17(3): p. 259-63.

42. Staal, Y.C., et al., Modulation of gene expression and DNA adduct formation in HepG2 cells by polycyclic aromatic hydrocarbons with different carcinogenic potencies. Carcinogenesis, 2006. 27(3): p. 646-55.

43. Hockley, S.L., et al., Time- and concentration-dependent changes in gene expression induced by benzo(a)pyrene in two human cell lines, MCF-7 and HepG2. BMC Genomics, 2006. 7: p. 260.

44. Lambert, C.B., et al., Dose- and time-dependent effects of phenobarbital on gene expression profiling in human hepatoma HepaRG cells. Toxicol Appl Pharmacol, 2009. 234(3): p. 345-60.

45. Lizarraga, D., et al., Benzo[a]pyrene-Induced Changes in MicroRNA-mRNA Networks. Chemical Research in Toxicology, 2012. 25(4): p. 838-849.

46. Goyak, K.M., et al., Expression profiling of interindividual variability following xenobiotic exposures in primary human hepatocyte cultures. Toxicol Appl Pharmacol, 2008. 231(2): p. 216-24.

47. Tateishi, T., et al., A comparison of hepatic cytochrome P450 protein expression between infancy and postinfancy. Life Sci, 1997. 61(26): p. 2567-74.

48. Rogue, A., et al., Comparative gene expression profiles induced by PPARgamma and PPARalpha/gamma agonists in human hepatocytes. PLoS One, 2011. 6(4): p. e18816.

49. Schulze, A., et al., Hepatocyte polarization is essential for the productive entry of the hepatitis B virus. Hepatology, 2012. 55(2): p. 373-83.

50. Zhang, L., et al., Systems Responses of Rats to Aflatoxin B1 Exposure Revealed with Metabonomic Changes in Multiple Biological Matrices. Journal of Proteome Research, 2010. 10(2): p. 614-623.

51. Shen, H.-M. and C.-N. Ong, Mutations of the p53 tumor suppressor gene and ras oncogenes in aflatoxin hepatocarcinogenesis. Mutation Research/Reviews in Genetic Toxicology, 1996. 366(1): p. 23-44.

52. Dhanasekaran, D., et al., Aflatoxins and Aflatoxicosis in Human and Animals. Aflatoxins - Biochemistry and Molecular Biology, ed. R.G. Guevara-Gonzalez. Vol. Chapter 12. 2011: InTech, Chapter 12.

53. Ayed-Boussema, I., et al., Effect of Aflatoxin B1 on Nuclear Receptors PXR, CAR, and AhR and Their Target Cytochromes P450 mRNA Expression in Primary Cultures of Human Hepatocytes. Int J Toxicol, 2012. 31(1): p. 86-93.

54. Antherieu, S., et al., Stable expression, activity, and inducibility of cytochromes P450 in differentiated HepaRG cells. Drug metabolism and disposition: the biological fate of chemicals, 2010. 38(3): p. 516-25.

55. Le Vee, M., et al., Differential regulation of drug transporter expression by all- 
trans retinoic acid in hepatoma HepaRG cells and human hepatocytes. Eur J Pharm Sci, 2013.

56. Rust, C. and G.J. Gores, Apoptosis and liver disease. The American Journal of Medicine, 2000. 108(7): p. 567-574.

57. Fabregat, I., Dysregulation of apoptosis in hepatocellular carcinoma cells. World J Gastroenterol, 2009. 15(5): p. 513-20.

58. Ricordy, R., et al., Impairment of cell cycle progression by aflatoxin B1 in human cell lines. Mutagenesis, 2002. 17(3): p. 241-9.

59. Crespi, C.L., et al., Development of a human lymphoblastoid cell line constitutively expressing human CYP1B1 cDNA: substrate specificity with model substrates and promutagens. Mutagenesis, 1997. 12(2): p. 83-9.

60. Gross-Steinmeyer, K., et al., Modulation of Aflatoxin B1-Mediated Genotoxicity in Primary Cultures of Human Hepatocytes by Diindolylmethane, Curcumin, and Xanthohumols. Toxicological Sciences, 2009. 112(2): p. 303-310.

61. Zhang, Y.J., et al., Silencing of glutathione S-transferase P1 by promoter hypermethylation and its relationship to environmental chemical carcinogens in hepatocellular carcinoma. Cancer Lett, 2005. 221(2): p. 135-43. 


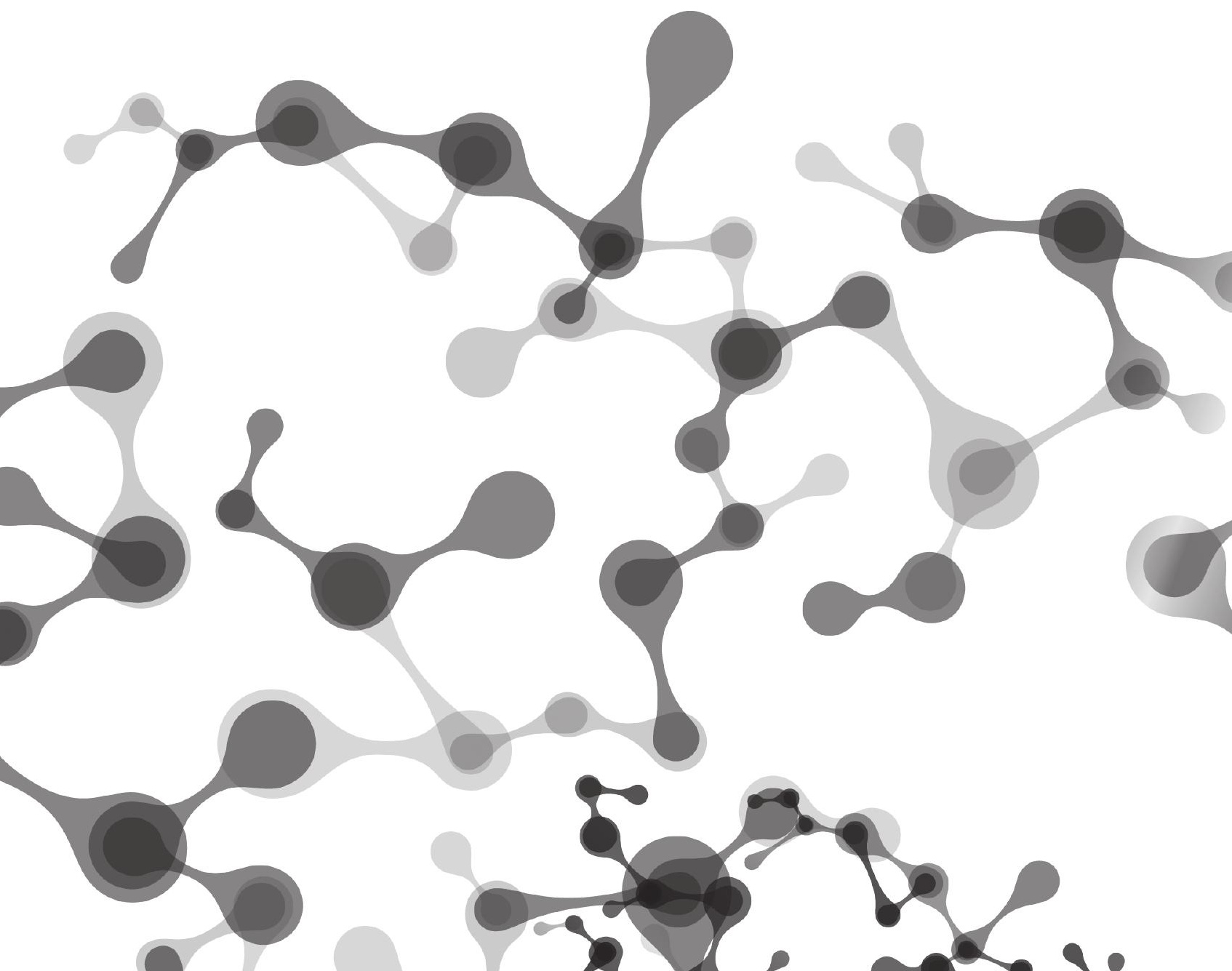




\section{Chapter 5 \\ Does the epigenome predispose for susceptibility to DNA damage?}

Marlon J.A. Jetten

Sandra M. Claessen

Hans Gmuender

Joost H.M. van Delft

Timo Wittenberger

Jos C.S. Kleinjans

In preparation 


\section{Abstract}

Although interindividual variation in responses towards chemical compounds, including carcinogens, is a well-known phenomenon, the causal factors for this variation are largely unknown. This complicates the issue of individual risk estimation. Interindividual variation in genemethylation has been proposed as an additional factor that may explain the differences in responses to compound exposure within the human population. This study focusses on determining to what extent baseline methylation status of a gene influences the response of that gene towards compound exposure. Special emphasis has been given to the evaluation of the DNA-damage response genes and on interindividual variation.

Primary human hepatocytes of several donors were exposed to aflatoxin B1 (AFB) or benzo( $\alpha$ )pyrene (BaP). The response to DNAdamage was evaluated by $\mathrm{\gamma H} 2 \mathrm{Ax}$-staining, these results were used to divide the study-population into two groups per compound; a low and a high responder group based on the benchmark dose for $\mathrm{\gamma H} 2 \mathrm{Ax}$ induction. For each of the sub-groups, genes that are unique in their expression responses were evaluated with respect to their baseline methylation status and baseline gene expression levels. Although clear differences between high and low responders to DNA-damage could be defined, baseline methylation status only seems to have marginal impact on induced gene expression levels. Interindividual variation in baseline gene expression levels does not seem to be related to the variation over individuals in baseline methylation levels. In turn, variation in baseline methylation was not reflected in compound-induced gene expression responses.

Thus, the overall conclusion is that the interindividual variation in baseline methylation status explains to only a limited degree the interindividual differences in susceptibility to carcinogen exposure of groups/individuals. Investigations of further (epi)genetic factors are required for a better understanding of regulatory mechanisms underlying interindividual variation in genotoxic responses. 


\section{Introduction}

It is a well-known fact that a large interindividual variation in responses to a wide variety of compounds, including chemical carcinogens, exists within the human population [1-3]. In order to explain the interindividual variation in toxic susceptibilities, environmental as well as genetic influences have been proposed as causative factors [1, 2]. While a broad range of studies has attempted to attribute interindividual variation to one single causative factor, the general conclusion is that interindividual variation seems to have a multi-factorial background [4]. Up till now, such causative factors for interindividual variation are still largely unknown.

Especially well studied, in the light of interindividual variation, are the Phase I metabolizing enzymes [4], particularly the expression and activity of cytochrome P450 enzymes. Many attempts have been taken to find a biological explanation underlying the variation in the human population concerning these enzymes, which among others, has led to the discovery of single nucleotide polymorphisms (SNPs) [5-9]. However, in general these SNPs can only explain part of the interindividual variation, which led to the conclusion that there must be additional regulatory mechanisms that are causal of this variation. DNAmethylation has been proposed as such a regulatory mechanism. DNAmethylation is considered a non-static system that can influence gene expression in response to environmental factors, without changes to the actual genetic code.

Recent technical progress led to the development of the methylation arrays, a chip-based system allowing for the fast analysis of the genome-wide methylation status [10]. The number of studies considering methylation has steadily increased, especially with respect to aging, cancer and immune-related diseases $[10,11]$.

DNA-damage is one of the hallmark processes related to the exposure to chemical carcinogens that, if not repaired correctly, can lead to carcinogenesis $[12,13]$. Interindividual variation in the response towards DNA-damage is a well-known phenomenon as well [14], and has been associated with the susceptibility towards carcinogenesis [6]. It is notoriously hard to assess an individual's susceptibility towards carcinogen exposure, because the molecular mechanisms that drive the process from DNA-damage via failure of repair to carcinogenesis, are still not fully understood. Yet, several SNPs have been suggested as possible causal factors for the interindividual differences in susceptibility 
to carcinogenesis $[6,8]$. Several SNPs allowed for the identification of more susceptible groups within the human population based on their correlations with biomarkers for carcinogen exposure.

Also, the epigenome has been suggested to be involved in carcinogenesis. Studies performing research on the methylation statusrelated changes in cancers, which can be considered as late stage endpoint of DNA-damage, have been performed $[15,16]$ Some of these studies investigated the human liver $[17,18]$, the major carcinogenmetabolizing organ. The cancer-related changes in gene methylation status thus suggest a role for the methylome in the process of tumor progression. It is therefore tempting to hypothesize that the methylome might also be involved in the initial DNA-damage response, a process already initiated long before actual tumor formation has occurred. Consequently, it may also be hypothesized that interindividual variation in baseline methylation levels of genes contributes to the interindividual variation in gene expression responses towards carcinogenic exposure. To evaluate this, primary human hepatocytes of several donors were exposed to two different carcinogens. Aflatoxin B1 (AFB) is a mycotoxin produced mainly by Aspergillus flavus which occurs naturally in our food chain [19-21]. Benzo( $\alpha$ )pyrene (BaP) may also occur in our food chain, but in general is a product of incomplete combustion of fossil fuels and also present in tobacco smoke [21-24]. Both compounds are known human carcinogens and are metabolized in the liver [24-26]. In this study, we focused on identifying genes of which the baseline methylation status can explain the response towards DNA-damage thereby discriminating between high and low responders.

Secondly, we set out to define those genes of which the interindividual variation in baseline methylation status can explain the interindividual variation in baseline gene expression and the interindividual variation in carcinogen-induced gene-expression responses. A $\gamma \mathrm{H} 2 \mathrm{Ax}$ staining technique was applied to evaluate the response to DNA-damage and results were used to define a low and a high-responder group for each of the two compounds. The benchmark dose (BMD) approach was used to model compound exposure over dose on an individual level. Methylation status, baseline gene expression and gene expression changes after compound exposure were determined using the appropriate array techniques allowing for genome wide evaluation. 


\section{Materials and Methods}

\section{Cell model and culture conditions}

Cryopreserved $\mathrm{PHH}$ of several individuals were purchased from Life technologies (Gibco, see Supplementary Table 1 for donor demographics and descriptive). A collagen sandwich was used to culture the cells in 12-well plates as described in the protocol provided by the supplier [27]. CHRM culture medium (Gibco) was used for thawing the cells, while William's medium E (WME)+Glutamax (Gibco) substituted with 10\% FCS (Gibco), 0.02\% penicillin/streptomycin (Gibco) and 0,1 $\mathrm{U} / \mathrm{ml}$ insulin (Invitrogen) was used for seeding/attaching the cells and WME+Glutamax substituted with $0.02 \%$ Pen/Strep, $0.1 \mathrm{U} / \mathrm{ml}$ insulin and $0.02 \mathrm{mg} / \mathrm{ml}$ hydrocortisone (Sigma-Aldrich) was used as medium for culturing/exposure of the cells. To ensure that the viability of the cells was in compliance with those listed by the supplier, viability was checked after thawing by a Trypan blue-exclusion test according to the supplier's protocol [27]. The hepatocytes were exposed to 1.25, 2.5 or $5 \mu \mathrm{M}$ AFB ( $n=6$ donors), $2.5,5$ or $10 \mu \mathrm{M} \mathrm{BaP}$ ( $n=8$ donor) or $0.5 \%$ DMSO (vehicle control) in culture medium for 24 hours. Since PHH of the various donors were expected to respond differently with respect to cytotoxicity, we choose to not focus on one single exposure-dose but include a dose-range instead. The dose-range was based on similar studies with PHH [28-31]. Data describing the similarities/differences in gene expression profiles of these PHH, HepG2 and HepaRG cells, both at baseline gene expression levels and in response to the above described compound-exposures were published before [32].

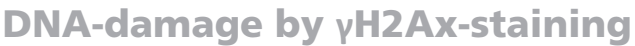

The experimental procedure for $\mathrm{y} \mathrm{H} 2 \mathrm{Ax}$-staining as described by Rothkamm et al. was applied to $\mathrm{PHH}$ from each donor cultured on cover slips and exposed to AFB, BaP or DMSO [33]. In short, cells were fixated and stained with the anti-phospho-histone $\mathrm{H} 2 \mathrm{Ax}$ (ser139) Clone JBW 301 antibody (Upstate Biotechnology, Lake Placid, New York) and Alexa fluor 488 goat anti-mouse IgG antibody (Invitrogen, Breda, The Netherlands). Foci were scored manually by assigning a DNA-damage score to each cell in the range from 0 (not damaged) to 4 (highly damaged). A minimum of 50 cells/dose/donor was scored.

The $\mathrm{yH} 2 \mathrm{Ax}$-staining data were used to split the donor population into two different groups, a low-responder group versus a highresponder group. The definition of these groups allows us to define 
whether baseline gene expression levels and/or baseline methylation status can explain the gene expression changes associated with the DNA-damage response in low versus high responder groups. Low versus high-responders were defined by a t-test applied on the BMD-values for each donor in the $\mathrm{y} \mathrm{H} 2 \mathrm{Ax}$-response for each compound separately. The BMD calculation procedure is described in further detail below.

\section{Transcriptomic sample preparation}

Total RNA isolation

$0.5 \mathrm{ml}$ QIAzol (QIAGEN) was used according to the manufacturer's protocol, to isolate total RNA from all samples. The miRNeasy Mini Kit (Qiagen) was used as instructed by the manufacturer to purify the RNA samples. Finally, RNA integrity was checked using the 2100 Bioanalyzer (Agilent) showing an average RIN of 8.

cDNA preparation/hybridization

The preparation of cDNA/cRNA has been described in detail elsewhere [34]. All samples were hybridized according to the manufacturer's recommended procedures on Affymetrix Human Genome U133A plus 2 GeneChips [34]. After hybridization the GeneChips were washed and stained using a fluidics station (Affymetrix) and scanned with a GeneArray scanner (Affymetrix).

PHH samples from donor nr. 3 and 4 exposed to AFB and the $2.5 \mu \mathrm{M}$ AFB exposed sample from donor nr. 1 did not perform well in the labeling procedure and were therefore excluded from further analyses.

\section{Transcriptomic data analysis}

Re-annotation, normalization and filtering

The Robust Multi-array Average (RMA) method was used for data normalization in the pipeline from ArrayAnalysis.org [35], after which the data were log2-transformed [36]. Data were re-annotated to an EntrezGenelD using the custom CDF V16.0.0 from BrainArray [37]. Genes with an average expression/dose lower than 6 (on a log2-scale) were excluded from further analysis. Furthermore, Present/Marginal/ Absent (PMA) tables were used to filter the data. PHH samples were filtered as a group ( $n=6$ AFB and $n=8$ for $B a P$, one sample for each donor) concerning the vehicle control samples (DMSO), allowing no more than $30 \%$ absent calls per gene. The list of genes resulting from this filtering step was used to study the baseline expression profiles from $\mathrm{PHH}$. For the PHH compound-treated samples (AFB and BaP) data on 3 
different doses/compound were available per donor. A gene showing more than 1 absent call within these 3 samples was excluded.

\section{Differentially expressed genes}

Differentially expressed genes (DEG) were calculated for either AFB or $\mathrm{BaP}$ (three replicates for each condition). A list of DEG genes was defined for each donor separately, thereby considering the samples from the three doses as replicates. First, log2-ratios between exposed cells and vehicle controls were calculated. DEG were determined based on the following criteria: A) a log2-ratio $<-0.58$ or $>0.58$ (i.e., absolute fold change $>1.5$ ) for the average of each experimental group, B) a log2-ratio $<-0.26$ or $>0.26$ (i.e., absolute fold change $>1.2$ ) for at least 2 replicates and, $C$ ) the same direction of the log2-ratio for all the samples within an experimental group, as described previously [34]. After this, two separate datasets were created. The first one allowed for the comparison on a group level (based on the outcome of the $\mathrm{yH} 2 \mathrm{Ax}$-staining, described in detail further above under 'DNAdamage by ${ }_{\mathrm{H}} \mathrm{H} 2 \mathrm{Ax}$-staining'), which consisted of the overlap of all the DEG responding in the same direction after AFB/BaP-exposure over individuals assigned to either low or high-responder group. The other dataset consisted of a union lists created by combining the list of DEG from the exposure response from each individual donor to one list/ compound.

\section{Calculation of the benchmark dose (BMD)}

BMDs were calculated from the compound-induced gene expression-response data for all of the genes with a highly variable baseline expression levels and from the data on the DNA-damage response ( $\mathrm{\gamma H} 2 \mathrm{Ax}$-stain). BMDExpress (version 1.4), a freely available program specifically developed to enable the calculation of benchmark doses from micro-array data, was used to define at which dose a compound exerts a predefined effect [38]. Data sets were created for each individual donor containing all the data of a compounds dose-range. The dose response modeling involves fitting the data to a selection of statistical models (power, linear, $2^{\circ}$ polynomial, $3^{\circ}$ polynomial and Hill models) and selecting the model that best describes the data with the least amount of complexity, this procedure is called the automated fitting procedure. In our case, the settings as recommended by the developers of the program were used. The automated fitting procedure was performed on the dose-response data from each donor separately. As such, each gene included in the analyses will receive a BMD which is specific for the compound-response of that 
gene in a particular donor. Thus, BMD analysis enables a dose-response of a gene to be summarized into one single value which is unique for an individual. By comparing the BMD's from one particular gene generated from the dose-response in $\mathrm{PHH}$ from different donors, one can thus determine the inter-individual variation in response to compound exposure for this gene.

\section{Methylation sample preparation}

For each of the donors, 2 samples were prepared for methylation analysis. One sample was taken just before the compounds were added to the cells; another one was taken 24 hours later (after exposure time). By analyzing these 2 samples as duplicates/donor, time effects on methylation, occurring during the 24 hour exposure timeframe, could be corrected for.

\section{DNA-purification and isolation}

The cells were collected using $500 \mu$ digestion buffer (Ambion), consisting of $1 \mathrm{mM}$ EDTA, $50 \mathrm{mM}$ Tris- $\mathrm{HCl}$ and $5 \%$ SDS at $\mathrm{pH} 8.0$ with the addition of proteinase $\mathrm{K}(1 \mathrm{mg} / \mathrm{ml}$, Ambion). The cells were incubated in the buffer for 1 hour at $55^{\circ} \mathrm{C}$ after which the proteinase $\mathrm{K}$ was inactivated at $80^{\circ} \mathrm{C}$. Then an RNAse treatment was applied to the cells for 1 hour at $37^{\circ} \mathrm{C}$ using $400 \mu \mathrm{g} / \mathrm{ml}$ RNAse A (Qiagen). Next phenol-chloroform-isoamylalcohol ( $\mathrm{PCl} ; 25: 24: 1 \mathrm{v} / \mathrm{v}$, Sigma) was added in a $1: 1$ ratio to the amount of sample material and manually mixed for $5 \mathrm{~min}$. The solution was separated in phases by centrifugation ( 5 min., max speed, $4^{\circ} \mathrm{C}$ ) after which the upper phase containing the DNA was collected. The $\mathrm{PCl} /$ centrifugation procedure was repeated until no more protein debris was visible in the interphase. The DNA was precipitated from the cleaned upper phase using $50 \mu \mathrm{l}$ M sodium acetate $\mathrm{pH} 5.6$ and $1250 \mu \mathrm{l} 100 \%$-ethanol $\left(30 \mathrm{~min} .-80^{\circ} \mathrm{C}\right)$. An additional washing-step was applied to the DNA pellet using $70 \%$ ethanol $\left(30\right.$ min. $-80^{\circ} \mathrm{C}$ ), after which the ethanol was again removed and the pellet was dissolved in $50 \mu l$ nuclease free water. The concentration of DNA was determined on the NanoDrop 1000 (Thermo Scientific, Waltham, MA), while making sure that the $260 / 280$ ratio was between $1.7-1.9$, and the $260 / 230$ was $>1.6$.

Methylated DNA Immunoprecipitation (MeDIP), Whole Genome Amplification (WGA) and methylation enrichment assessment

Fragmented genomic DNA was obtained by sonication to obtain fragments ranging from 200 base pairs- 1000 base pairs. Then the fragmented DNA was cleaned using silica columns (Zymo Research) 
using TE buffer for elution. Next, $4.4 \mu \mathrm{g}$ of each fragmented sample was spiked with $40 \mathrm{ng}$ of a positive (methylated) control and $40 \mathrm{ng}$ of a negative (un-methylated) control in a total volume of $495 \mu \mathrm{LTE}$ buffer [39]. For each of the prepared mixtures, $10 \%$ of the volume was kept aside as input (IP)-sample at $4^{\circ} \mathrm{C}$. The remainder of the sample, from now on named the MeDIP-sample, was immuno-precipitated as previously described using $12 \mu \mathrm{L}$ monoclonal antibody against $5^{\prime}$ methylcytidine (Eurogentec) $[40,41]$. Subsequently, MeDIP-samples were purified using silica columns (Zymo Research) applying TE buffer for elution. Both IP and MeDIP-samples were subjected to 2 WGA steps. For the first step the WGA2 kit (Sigma Aldrich) using $40 \mathrm{ng}$ input material was applied. For the second step 25ng input material from the first WGA round was added to the WGA3 kit (Sigma). The manufacturer's instructions were followed, but the DNA-fragmentation step was omitted since the DNA was already fragmented before by sonication. WGA reactions were cleaned up using silica columns (Sigma Aldrich) and eluted in water. Finally, the enrichment of methylation in paired samples (IP/MeDIP) was determined by qPCR. Enrichment was defined as the ratio of positive control/negative control, applying the $\triangle \triangle \mathrm{Cq}$ method. For this, primers, previously described by Lisanti et al. were used: positive control forward: TAC AGA AAG ACG GAC GAA GG and reverse: TGG TGG GCG TTT TCA TAC AT; negative control forward: TTC GTG ATA TTC CGT CGC TG and reverse: AGT TTT TTG CCG CTT TAC CG [39].

\section{MeDIP-Chip array}

DNA methylation levels were determined using the Human DNA Methylation 2.1M Deluxe Promoter Array (Roche NimbleGen). The manufactures' protocol was used for the labeling and hybridization procedures. Washing of the slides was performed using the the NimbleGen wash buffer kit. The NimbleGen MS 200 micro array scanner with $2 \mu \mathrm{m}$ high resolution was used for scanning.

\section{Methylation data analysis}

Annotation, normalization and filtering

Annotation was performed with the software provided with the MeDIP-Chip arrays, using annotation file version 100929-HG19_Deluxe_ Prom_Meth_HX1_2011. Data were re-annotated to an EntrezGenelD using the custom CDF V16.0.0 from BrainArray [37]. Then data were normalized using the LOWESS normalization procedure and a log2 transformation was applied in the Expressionist software provided by Genedata. Next, M-values (log intensity ratios) were calculated for 
each sample. Finally, the 2 samples/donor were averaged and the data for each donor were mapped onto the human genome (hg19) using expressionist.

Identification of methylation-status

To define the methylation status of genes a sliding ANOVA was applied with a window size of $500 \mathrm{bp}$. About 5 probe signals were present within a window, since the length of the probes was approximately 50 base pairs. The output value of a sliding ANOVA represents a Phred-scaled ANOVA p-value for a specific position. Significant ranges were determined as having a Phred-scaled $p$-value $\geq 30$. Next, significant ranges were annotated with genes if these internal ranges could be annotated by the name of the matching entity in annotation file. The tolerance distance for this annotation was 1 kilobase downstream and 3 kilobases upstream (for clarification see Supplementary Figure 1).

\section{Data integration}

Analysis on the group level

As described before, the donor population was split in a low and high responder group for each compound based on the DNA-damage response as determined by $\mathrm{\gamma H} 2 \mathrm{Ax}$-staining. Next, for each of the groups the overlap between donors in DEG with a similar direction of response was determined. Genes that were unique for either the low or the high responder group were selected per compound. Then, the genes selected for each subgroup, were used for pathway analysis (further details below under 'Biological interpretation'). All genes that could be assigned to a pathway were taken from this analysis, resulting in a list of biologically relevant response-genes for the low-responder and the high-responder group for each compound. For all of these biologically relevant response genes, the baseline methylation status was compared between responder groups. Consequently, base line gene expression levels of such differentially methylated genes were determined.

Analysis on the individual level

This analysis was performed to answer the question whether there are any genes for which the interindividual variability in change in expression after compound exposure can be explained by the variability in the baseline methylation/expression status of that gene across donors studied. Therefore, as a first step, baseline gene methylation levels were analyzed to define those genes with a high baseline variation (standard deviation > 0.5) in methylation levels between individuals. An identical 
procedure was followed to determine those genes with a high variability in baseline gene expression level between individuals.

Then, for each of the selected genes, baseline gene expression status and the gene expression response after carcinogen exposure (BMD-value) were determined for each individual. After that, methylation gene-expression status, baseline gene expression levels and BMD for carcinogen-induced gene expression-responses were imported into the Analyst package of the Genedata software. A Spearman correlation (ranking based on donors) was performed for each gene considering the following associations: baseline gene expression-level vs baseline methylation status, baseline gene expression level vs BMD from gene expression response after AFB exposure, baseline gene expression level vs BMD for gene expression-response after BaP exposure, baseline methylation status vs BMD for gene expression-response after AFB exposure, and baseline methylation status vs BMD for gene expression response after BaP exposure. Only correlations with a coefficient $>0.7$ were taken into account. These correlations were then used to identify genes for which the inter-individual variation in response to carcinogen exposure can be explained by the variation between individuals at baseline methylation status and by the interindividual variation in the baseline gene expression levels of that gene. A Venn-diagram was used to determine overlap.

\section{Biological interpretation}

Over-representation analyses performed in the freely-available, online tool ConsensusPathDB enabled biological interpretation of the data [42].

In discriminating between high and low responders, this analysis was applied in order to determine biologically relevant genes in expression responses. DEG specific for the response in low-responders or in high-responders, were loaded into the over-representation part of the tool using a background list composed of all defined DEG in response to a compound (specific for a group or not).

An over-representation analysis was also used to define the biological function of all genes with a high interindividual variability in baseline methylation status or baseline gene expression levels. These genes were analyzed against a background list consisting of all genes that past the filtering criteria as described under 'Annotation, normalization and filtering' for the methylation data or under 'Reannotation, normalization and filtering' for the transcriptomic data. All 
gene lists resulting from performed correlation analyses, were fed to ConsensusPathDB with a background list identical to the list of genes showing a high interindividual variability in baseline gene expression levels. The tool was applied using the recommended standard settings for all parameters, pathways with a $p$-value $<0.05$ being considered significant. All output lists of pathways were summarized by assigning each pathway to a functional category.

\section{Results}

\section{Analysis on the group level}

To detect DNA-damage response-associated genes which baseline expression levels and baseline methylation status are indicative for the expression changes of that gene after carcinogen exposure, the donor population was divided into a low-responder group and a high-responder group per compound based on established $\mathrm{H}_{2} \mathrm{Ax}$-stain $B M D$, by performing a t-test between groups (AFB $p$-value $=0.009$, for $B a P$-value $=0.049$ ). For $A F B$, the low-responder group was formed by donor 7, 10 and 11 while donor 3, 4 and 12 were assigned to the highresponder group. Donor 3, 4, 7 and 10 and donor 5, 6, 11 and 12 were assigned to the low-responder and high-responder group respectively for BaP.

An overlap of 194 DEG (32 up-regulated and 162 downregulated) were uniquely identified in donors assigned to the lowresponder group after AFB exposure. For the high-responder group to AFB, 907 DEG were uniquely defined (459 up-regulated and 448 downregulated). In the low-responder to $\mathrm{BaP}$, a total of $20 \mathrm{DEG}$ was defined (18 up-regulated, 2 down-regulated) while for the high responder group no common DEG could be defined that are unique for this group. An overview of these gene lists can be found in Supplementary Table 2.

A pathway analysis was performed to enable the selection of DEGs possessing a clearly defined biological function in response to carcinogen exposure. This resulted in $22 \mathrm{DEGs}$ selected for the AFB low-responders, 79 DEGs in AFB high-responders and 15 DEGs in BaP low-responders (also see Supplementary Table 2). A summary showing the functional categories to which the pathways belong is presented in Table 1, part A, a full list of all pathways related to the responses for each group, is shown in Supplementary Table 3. 
Table 1; Categorical summary of pathway analysis

Categorical description based on biological function of pathways detected by over-representation analysis using ConsensusPathDB ( $p$-values<0.05) on respectively; A) genes specific for the lowresponder group to $A F B$, genes specific for the high-responder group to $A F B$, genes specific for the low-responder group to $B a P$ and $B$ ) genes with a interindividual variability in baseline methylation levels

\begin{tabular}{|l|l|l|l|}
\cline { 2 - 4 } \multicolumn{1}{c|}{} & \multicolumn{2}{l}{ A) Count of category response } \\
\hline Category & $\begin{array}{l}\text { AFB low- } \\
\text { responders }\end{array}$ & $\begin{array}{l}\text { AFB high- } \\
\text { responders }\end{array}$ & $\begin{array}{l}\text { BaP low- } \\
\text { responders }\end{array}$ \\
\hline Apoptosis & 1 & 2 & 2 \\
\hline Cell component & 0 & 0 & 0 \\
\hline Cell cycle & 1 & 6 & 6 \\
\hline DNA-damage/stress & 0 & 0 & 6 \\
\hline Drug/Compound & 1 & 15 & 6 \\
\hline $\begin{array}{l}\text { Drug/Compound and } \\
\text { metabolism }\end{array}$ & 0 & 0 & 6 \\
\hline Gene expression & 0 & 4 & 3 \\
\hline Haemostasis & 0 & 0 & 0 \\
\hline Human diseases & 0 & 14 & 7 \\
\hline Immune & 1 & 0 & 0 \\
\hline Metabolism & 0 & 8 & 26 \\
\hline Neuronal system & 0 & 0 & 0 \\
\hline Organismal Systems & 0 & 0 & 1 \\
\hline Receptor & 0 & 0 & 0 \\
\hline Signaling & 4 & 2 & $\mathbf{7 1}$ \\
\hline Transport & 0 & 0 & $\mid$ \\
\hline Grand Total & $\mathbf{8}$ & & $\mid$ \\
\hline
\end{tabular}

\begin{tabular}{l|l|}
\hline $\begin{array}{l}\text { B) Count of category } \\
\text { baseline variable genes } \\
\text { Baseline } \\
\text { methylation }\end{array}$ & $\begin{array}{l}\text { Baseline } \\
\text { gene } \\
\text { expression }\end{array}$ \\
\hline 0 & 1 \\
\hline 0 & 8 \\
\hline 1 & 4 \\
\hline 0 & 2 \\
\hline 4 & 77 \\
\hline 0 & 7 \\
\hline 3 & 2 \\
\hline 1 & 11 \\
\hline 2 & 21 \\
\hline 3 & 19 \\
\hline 2 & 97 \\
\hline 10 & 0 \\
\hline 3 & 1 \\
\hline 0 & 1 \\
\hline 8 & 267 \\
\hline 1 & 14 \\
\hline $\mathbf{3 8}$ & \\
\hline
\end{tabular}

Next, for all of the biologically relevant genes, directionality of the gene expression response, baseline methylation levels and baseline gene expression status was compared in all predefined groups. Concerning the low-responder group to AFB, 22 DEGs were selected as biologically relevant. Of these 22 DEGs, 2 genes (CEBPD and SREBF1, see Table 2: genes highlighted in dark gray) had a difference in baseline methylation status between the low-responder and the high- responder group, but these genes did not show a difference in baseline gene expression status between groups. The other way around, there was one gene (CYCS) with a difference in baseline gene expression status between groups, however no difference in methylation levels between groups could be defined for this particular gene. The same comparisons as above, were made for the high-responder group to AFB. Of the 
79 DEGs that were biologically relevant, 4 genes (POLR2K, TBL1X, SEC24A and ELOVL1) had a difference in baseline methylation levels between groups, but these did not display a difference in baseline gene expression status. GNG11 and HIST1H2AE are two genes with a difference in baseline gene expression status between groups, but no difference in baseline methylation levels between groups could be found for these genes. Finally, in the BaP low-responder group (15 biologically relevant DEGs), ALDH3A1 showed a difference in baseline gene expression status between groups, but no difference in baseline methylation status. In fact, none of the 15 DEGs showed a difference in baseline methylation status between groups.

In addition, several genes within the list of biologically relevant DEGs over all groups could be defined that exhibited baseline hypermethylation versus gene silencing in baseline gene expression (Table 2: striped in gray) or hypo-methylation associated with baseline gene expression (highlighted in light gray). However, since these effects are not specific to one of the responder groups, these genes do not represent candidates for explaining the difference in susceptibility to the DNA-damage response between groups.

A full overview of the directionality of the gene expression response, baseline methylation levels, and baseline gene expression status for all of the biologically relevant DEGS, including those without any difference in baseline methylation status or baseline gene expression status between groups, is provided in Supplementary Table 4.

\footnotetext{
Table 2; Summary of comparison between baseline gene expression status, baseline methylation level and compound-induced gene expression response

Genes specific for either the low or high responders towards DNA-damage (based on $\mathrm{\gamma H}_{2} \mathrm{Ax}$ staining) were selected, only genes found to have aberrant baseline gene expression/ methylation levels between groups are shown in this table. Direction gene response either - (down-regulated) or + (up-regulated); baseline gene methylation -1 (hypo-methylated), 1 (hyper-methylated) or ... (not aberrantly methylated from background or no information available on chip); baseline gene expression either 1 (expressed) or 0 (not expressed). Dark gray: aberrant baseline gene expression/ methylation levels at group level, light gray: no baseline gene expression and baseline hyper-methylation (no difference between groups), striped: baseline gene expression with baseline hypo-methylation (no difference between groups).
} 


\begin{tabular}{|c|c|c|c|c|c|c|}
\hline \multicolumn{3}{|c|}{ AFB low-responder group } & \multicolumn{2}{|c|}{$\begin{array}{l}\text { Baseline gene } \\
\text { methylation }\end{array}$} & \multicolumn{2}{|c|}{ Baseline gene expression } \\
\hline EntrezGenelD & Gene name & $\begin{array}{l}\text { Direction gene } \\
\text { response }\end{array}$ & $\begin{array}{l}\text { Low- } \\
\text { responders }\end{array}$ & $\begin{array}{l}\text { High- } \\
\text { responders }\end{array}$ & $\begin{array}{l}\text { Low- } \\
\text { responders }\end{array}$ & $\begin{array}{l}\text { High- } \\
\text { responders }\end{array}$ \\
\hline 1052 & CEBPD & - & -1 & 1 & 1 & 1 \\
\hline 1387 & CREBBP & - & -1 & -1 & 1 & 1 \\
\hline 5770 & PTPN1 & - & -1 & -1 & 1 & 1 \\
\hline 6720 & SREBF1 & - & -1 & 1 & 1 & 1 \\
\hline 54205 & CYCS & + & $\ldots$ & $\ldots$ & 1 & 0 \\
\hline \multicolumn{3}{|c|}{ AFB high-responder group } & \multicolumn{2}{|c|}{$\begin{array}{l}\text { Baseline gene } \\
\text { methylation }\end{array}$} & \multicolumn{2}{|c|}{ Baseline gene expression } \\
\hline EntrezGenelD & Gene name & $\begin{array}{l}\text { Direction gene } \\
\text { response }\end{array}$ & $\begin{array}{l}\text { Low- } \\
\text { responders }\end{array}$ & $\begin{array}{l}\text { High- } \\
\text { responders }\end{array}$ & $\begin{array}{l}\text { Low- } \\
\text { responders }\end{array}$ & $\begin{array}{l}\text { High- } \\
\text { responders }\end{array}$ \\
\hline 126 & ADH1C & - & -1 & -1 & 1 & 1 \\
\hline 132 & ADK & - & -1 & -1 & 1 & 1 \\
\hline 1543 & CYP1A1 & + & -1 & -1 & 1 & 1 \\
\hline 1577 & CYP3A5 & - & -1 & -1 & 1 & 1 \\
\hline 2791 & GNG11 & + & -1 & -1 & 1 & 0 \\
\hline 3012 & HIST1H2AE & + & $\ldots$ & $\ldots$ & 0 & 1 \\
\hline 3014 & H2AFX & + & -1 & -1 & 1 & 1 \\
\hline 3615 & IMPDH2 & + & -1 & -1 & 1 & 1 \\
\hline 4047 & LSS & - & -1 & -1 & 1 & 1 \\
\hline 4067 & LYN & - & -1 & -1 & 1 & 1 \\
\hline 5105 & PCK1 & - & -1 & -1 & 1 & 1 \\
\hline 5440 & POLR2K & + & 1 & -1 & 1 & 1 \\
\hline 6907 & TBL1X & - & -1 & 1 & 1 & 1 \\
\hline 8336 & HIST1H2AM & + & 1 & 1 & 0 & 0 \\
\hline 10735 & STAG2 & - & -1 & -1 & 1 & 1 \\
\hline 10802 & SEC24A & - & 1 & -1 & 1 & 1 \\
\hline 11059 & WWP1 & - & -1 & -1 & 1 & 1 \\
\hline 51141 & INSIG2 & - & -1 & -1 & 1 & 1 \\
\hline 64834 & ELOVL1 & + & -1 & 1 & 1 & 1 \\
\hline 85236 & HIST1H2BK & + & -1 & -1 & 1 & 1 \\
\hline \multicolumn{3}{|c|}{ BaP low-responder group } & \multicolumn{2}{|c|}{$\begin{array}{l}\text { Baseline gene } \\
\text { methylation }\end{array}$} & \multicolumn{2}{|c|}{ Baseline gene expression } \\
\hline EntrezGenelD & Gene name & $\begin{array}{l}\text { Direction gene } \\
\text { response }\end{array}$ & $\begin{array}{l}\text { Low- } \\
\text { responders }\end{array}$ & $\begin{array}{l}\text { High- } \\
\text { responders }\end{array}$ & $\begin{array}{l}\text { Low- } \\
\text { responders }\end{array}$ & $\begin{array}{l}\text { High- } \\
\text { responders }\end{array}$ \\
\hline 218 & ALDH3A1 & + & 1 & 1 & 0 & 1 \\
\hline 1543 & CYP1A1 & + & -1 & -1 & 1 & 1 \\
\hline 9518 & GDF15 & + & -1 & -1 & 1 & 1 \\
\hline
\end{tabular}




\section{Analysis on the individual level}

Over-representation analyses on the genes with high variability in baseline methylation levels revealed pathways mainly involved in 'neuronal system' and 'signaling'-related processes (see Supplementary Table 3 A and Table 1, part B). The same analysis but then on those genes with a high interindividual variability in baseline gene expression levels, resulted in pathways mainly involved in metabolism (cytochrome P450, phase I/II metabolism), immune-related processes and several pathways related to haemostasis (see Supplementary Table 3 B and Table 1, part B).

A full list of all of the genes with a high interindividual variation in baseline methylation status/baseline gene expression levels (involved in any pathway or not) is provided in Supplementary Table $5 \mathrm{~A}$ and $\mathrm{B}$ respectively.

The donor-ranked Spearman analysis of the baseline methylation status of these variable genes in association with their baseline gene expression levels revealed a relevant correlation for 12 genes (1 positive, 11 negative, see Table 3; Meth ranges vs Tx).

Analogue to this, correlation between the BMDs calculated from the compound-induced gene expression response and the baseline methylation levels of these genes showed 29 correlations (17 positive, 12 negative) for $A F B$, and 17 correlations (8 positive, 9 negative) for $\mathrm{BaP}$ (Table 3; Meth vs AFB BMD and Meth vs BaP BMD respectively).

Additionally, the correlation analyses between baseline gene expression levels of variable genes and their corresponding BMDs calculated from the compound-induced gene expression responses, resulted in 51 correlations ( 21 positive, 30 negative) for AFB, and 25 correlations (10 positive, 15 negative) for BaP (Table 3; Tx vs AFB BMD and Tx vs BaP BMD respectively).

\section{Table 3; Correlation on interindividual variation}

A Spearman's correlation analysis was performed by ranking the data based on individuals. The following comparisons were made; baseline methylation status of highly variable genes (standard deviation > 0.5) to baseline gene expression levels (Meth ranges vs TX), baseline gene expression levels to $B M D$ s derived from the compound induced gene-expression response (TX vs XXX BMD, AFB for Aflatoxin B1 and BaP for Benzo(a)pyrene) and baseline methylation levels to BMDs (Meth vs XXX $B M D$ ). Only genes with a correlation coefficient (cor. coef.) $>0.7$ or $<-0.7$ are shown. 
๑ัँ

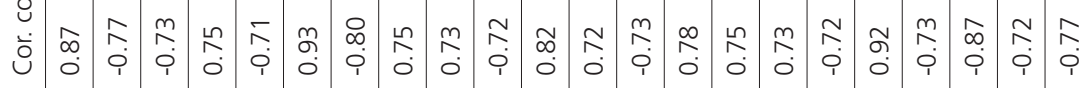

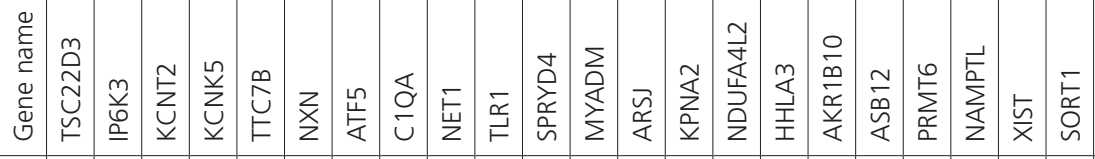

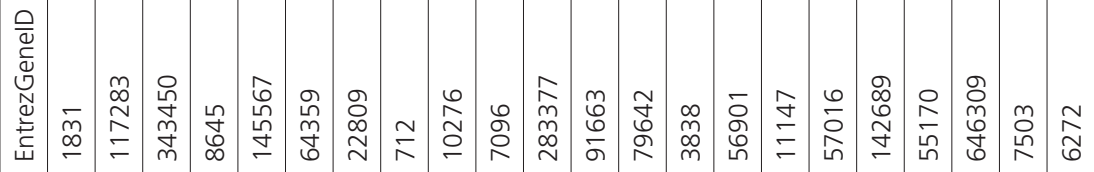

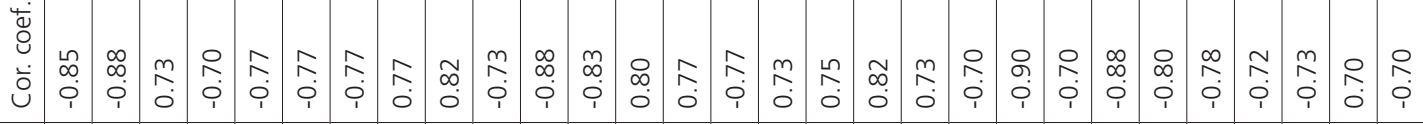

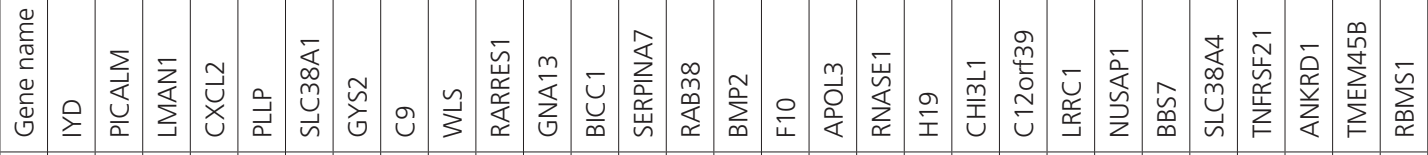

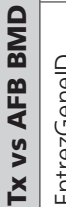

ฮั

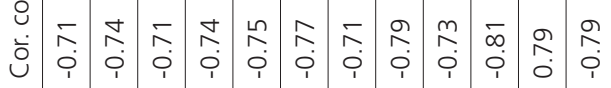

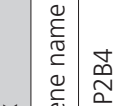

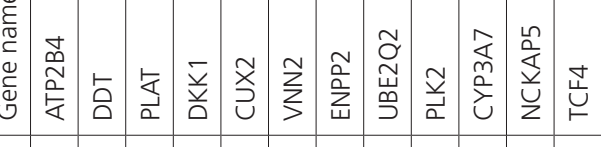

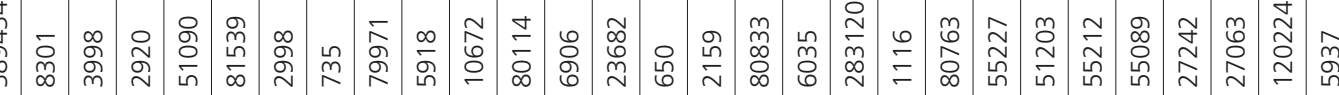


这

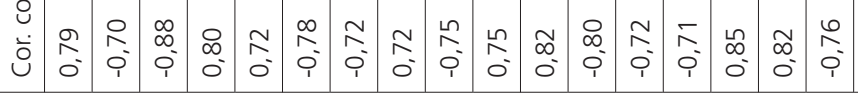

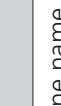

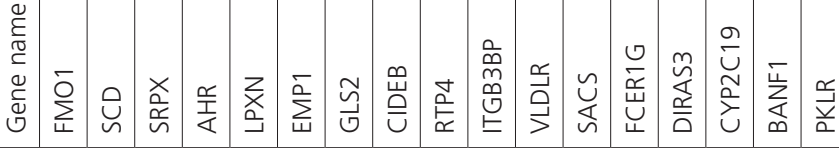

s.

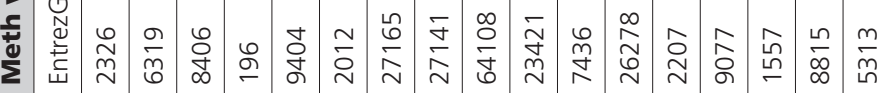

这

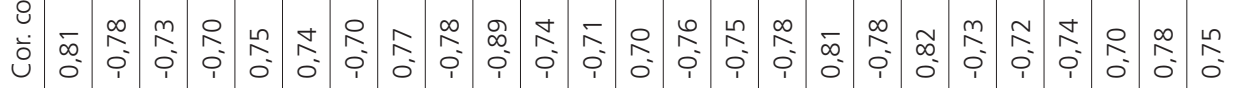

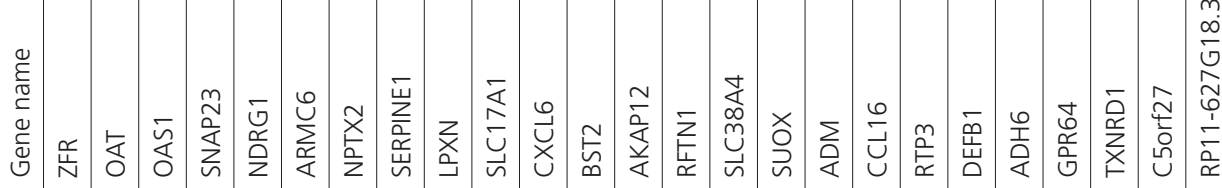

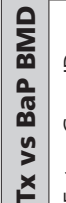

$\frac{0}{\bar{v}}$

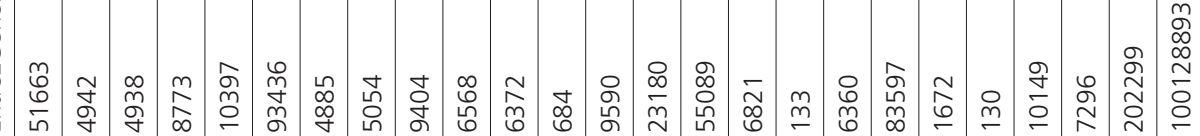

巡

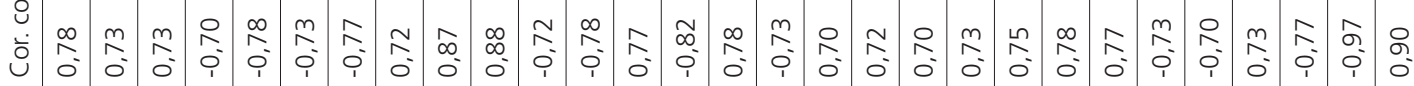

产

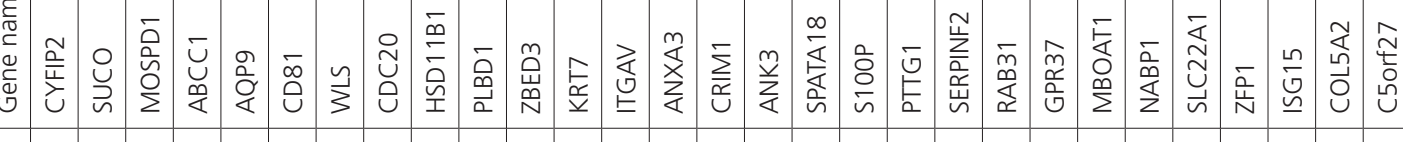

일

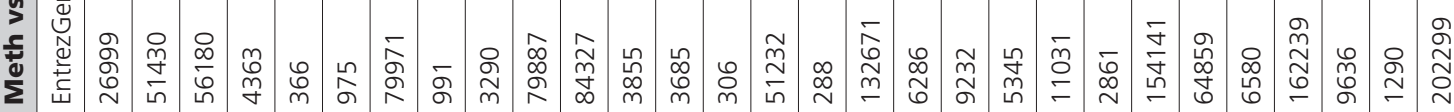


Finally, to find those genes for which the interindividual variation in response to carcinogen exposure can be explained by the variation between individuals in baseline gene expression/methylation levels, an overlap was created between all the correlation analyses mentioned above (see Figure 1). Figure 1 was created without any pre-selection for the directionality of the correlation coefficient, since the directionality only needs to be taken into consideration for those genes that show an overlap, which according to the Venn-diagram is very limited.
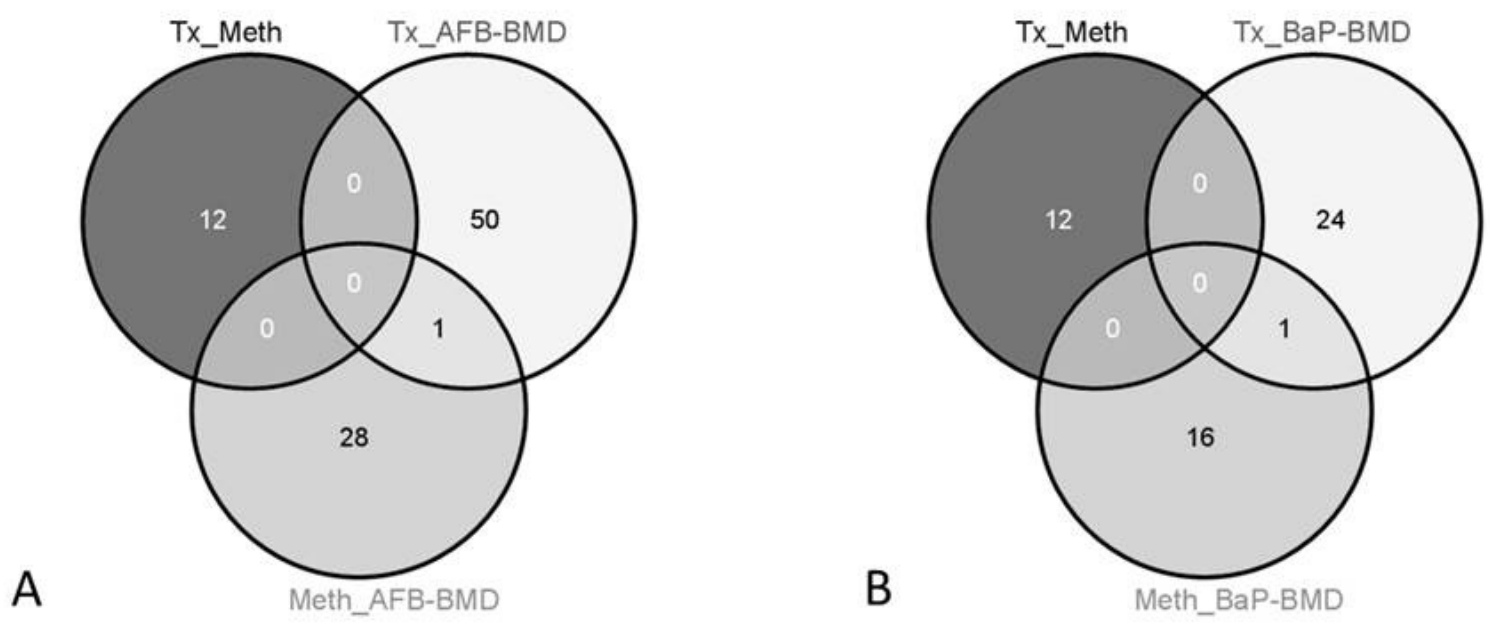

Figure 1; Overlap in correlations on interindividual variation

Overlap in the correlations between baseline gene-expression level and baseline gene methylationstatus (TX_Meth), baseline gene-expression level and BMDs from compound induced gene-expression response (TX_XXX-BMD) and baseline gene methylation-status and BMDs from compound induced gene-expression response (Meth_XXX-BMD) for A) Aflatoxin B1 (AFB) and B) Benzo(a)pyrene (BaP).

For both compounds, 1 gene shows overlap between two of the input lists, being the correlations between baseline methylation levels vs. gene expression response and between baseline gene expression levels vs. gene expression response.

For AFB the overlapping gene is WLS, with a negative correlation for the correlation between baseline methylation vs gene expression responses and a positive correlation between baseline gene expression vs gene expression responses.

LPXN is the only overlapping gene in the analysis for BaP. LPXN has a positive correlation between its baseline methylation status vs gene expression response and a negative correlation between baseline gene expression level vs gene expression response. A visualization of the correlations for these two genes is provided in Figure 2. In this figure 
each of the input parameters for the correlation is depicted by a block, e.g. baseline methylation status, baseline gene expression status or gene expression response. The gradient/triangle underneath each of the blocks represents the ranking of the donors for that specific parameter. A positive correlation between two parameters/blocks dictates that the ranking for the donors/direction of the triangles in the two correlated parameters/blocks is similar. A negative correlation thus dictates an inverse of the ranking/directionality of the triangle.
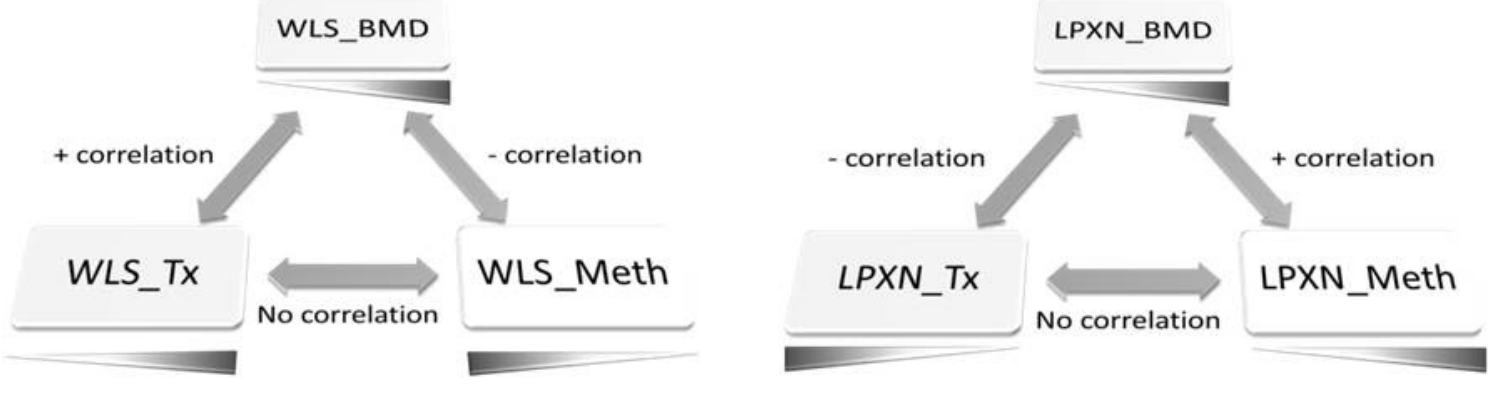

Figure 2; Directional comparison of correlation coefficient of genes with overlap in interindividual variation

Genes with overlap in the correlations between baseline gene-expression level and BMDs from compound induced gene-expression response (XXX_TX and XXX_BMD) and baseline gene methylation-status and BMDs from compound induced gene-expression response (XXX_Meth and XXX_BMD) for A) WLS (EntrezGeneID: 79971) after Aflatoxin B1 exposure and B) LPXN (EntrezGenelD: 9404) after Benzo( $\alpha$ )pyrene exposure. The gradient in the triangle represents the distribution of baseline gene-expression (XXX_TX)/baseline methylation-status (XXX_Meth)/BMD (XXX_BMD) over donors ranging from low (white) to high (black). 


\section{Discussion}

In this study, we aimed to determine whether variation in baseline methylation status of genes is indicative for the variation in gene expression response levels upon carcinogen exposure. Therefore, $\mathrm{PHH}$ of multiple donors were exposed to a dose-range of AFB or BaP for 24 hours. An analysis on group level was performed based on a low versus high response to carcinogen-induced DNA-damage (determined by $\mathrm{H}_{2} \mathrm{~A}$ - -staining). This approach enabled the detection of genes that, based on their baseline methylation status, distinguish between low-responders or high-responders to DNA-damage. In addition, we searched for an association between interindividual variation in baseline methylation-status/gene-expression level and the variation between individuals in gene-expression responses induced by compound exposure.

Low and high-responders with a statistically significant difference in the response towards DNA-damage assessed by $\mathrm{\gamma H} 2 \mathrm{Ax}$ staining could be defined within the population used in this study. The phenomenon of interindividual variation in response to DNA-damage as is described in literature thus seems to apply to the effects caused by AFB or BaP exposure within the current study population [43]. A gene expression response to AFB-exposure, that is specific for the low-responding group, could be defined, as well as a gene expression response to $A F B$ that is unique to the high-responding group. For the gene expression response to $\mathrm{BaP}$ exposure, a set of genes exclusive to the low-responder group could be defined, but no such gene set existed in the high-responder group. Since the overall gene expression response to AFB included more genes that the response to $\mathrm{BaP}$, this was to be expected.

The pathway analysis of expressed genes specific for the AFB related low-responder group resulted in the identification of several 'apoptosis', 'cell cycle', 'drug/compound', 'immune' and 'signaling'related pathways (Table 1, Supplementary Table 3). Although all before mentioned pathway-categories seem plausible in view of a DNAdamage response, the number of pathways was somewhat limited and no clear process could be distinguished.

On the other hand, pathway analysis of gene expression responses modified in the AFB high-responder group, did show a clearly defined response. The category 'metabolism' is entirely composed of pathways involved in cholesterol/fatty acid-related processes (Table 
1, Supplementary Table 3). Exposure of rats to AFB has been shown to cause an increase in lipids in the liver (mainly phospholipids and cholesterol) with an associated decrease in triglycerides [44].

However, even more importantly, AFB has been shown to cause the formation of reactive oxygen species (ROS) such as superoxide radicals, hydroxyl radicals and hydrogen peroxides, which contribute to the cellular damage caused by AFB [45]. In addition, within the functional category defined as 'human disease' several pathways related to cholesterol/fatty acids aberrations could be defined for instance, 'Cholesteryl ester storage disease' and 'hypercholesterolemia'. The functional category 'drug/compound' largely consisted of a group of drugs related to the class of statins, for instance Lovastatin and Pravastatin, which are used for the regulation of cholesterol levels $[46,47]$. In summary, the high-responder group to AFB seems to be characterized by a response that involves fatty acids and their metabolism. This response might be indicative of a cellular stressresponse that could lead to cell death and/or additional DNA-damage. AFB be is known to exert both genotoxic and cytotoxic effects which corresponds with the above described processes

Thus, different genes/pathways characterize the responses of the low and high-responder groups to AFB. However, these responses do not seem to have an obvious link with the DNA-damage response. Nevertheless, our previously published data did show a clear response towards DNA-damage for these cells [32]. It therefore seems that the DNA-damage response is a shared response between both the low and high-responder group that with the statistical approach as applied in this manuscript is not taken into consideration. In addition, there was no actual significant difference in $\mathrm{yH} 2 \mathrm{Ax}$ foci between groups (based on a t-test between groups, data not shown). Still, cells from individuals demonstrating a DNA-damage response after higher BaP-doses are the ones affected by a disruption in their fatty acid metabolism. This response could apparently be prevented in the low-responders. Since the above described responses in general have a negative effect on the cell, or are triggered in instances of an adverse cellular event, it seems that the high-responder group seems more susceptible to the toxic effects of AFB.

A gene response for the low-responders to $\mathrm{BaP}$ could also be defined. The functional categories 'apoptosis', 'cell cycle', 'DNAdamage/stress', 'drug/compound and metabolism', 'gene expression' and 'metabolism' all include pathways directly related to compound 
metabolism and the DNA-damage response (see Table1, Supplementary Table 3). Pathways related to compound metabolism include 'phase 1 functionalization of compounds', 'cytochrome P450', 'xenobiotics metabolism', 'arylhydrocarbon receptor (AhR) signaling pathway' and 'benzo(a)pyrene metabolism'. Cytochrome P450 (CYP) enzymes, including CYP1A1, CYP1A2 and CYP1B1 are major enzymes responsible for the bio-activation of $\mathrm{BaP}$ and are all under regulation of the arylhydrocarbon receptors [48-50]. The pathways associated to the DNA-damage response include several tumor protein 53 (p53) and cell cycle-related pathways. p53 Is known to be a vital regulator of the DNAdamage response which consists of a balance between the repair of damage or cell death by influencing the cell cycle progression [51, 52]. In addition the functional category 'human disease' consists entirely of cancer-related diseases, including 'chemical carcinogenesis'. Thus, for the BaP low-responder group, a clear DNA-damage response-related gene expression profile could be defined. The low-responder group is characterized by individuals whose cells exert a response at a lower BaPdose than the individuals from high-responder for the above mentioned genes/pathways. This could mean that the low-responders suffer from more DNA-damage at a lower BaP dose and therefore need an earlier DNA-damage response than the high-responders. However, the actual average number of foci was not significantly higher in the lowresponder group (based on t-test between low/high responder groups, data not shown). Therefore is seems that the low-responder group is simply more efficient than the high-responder group in inducing the DNA-damage gene expression response.

Next, we evaluated whether differences in gene expression responses between low and high-responder groups can already be seen at baseline gene methylation levels. And, if these differences appear present in baseline methylation levels, whether these differences in baseline methylation levels are reflected by the variation in baseline gene expression status between groups. Thus, we hypothesize that if a gene shows a response unique to the low responder group, this gene is apparently available for transcription in this low responder group, but not in the high responder group. Biology then dictates that this particular gene should be hypo-methylated in the low-responder group where it shows a response, but should be hyper-methylated and as such silenced in the high-responder group. An overview of all genes that were detected as being biologically relevant in one of the response groups and that had opposing directionality of their baseline methylation levels between responder groups are shown in Table 2 (highlighted in dark gray). 
In the AFB low-responder group, there are 2 genes with a difference in baseline methylation levels between responder groups. CEBPD (full name: CCAAT/enhancer binding protein) and SREBF1 (full name: sterol regulatory element binding transcription factor 1) are both transcription factors. While CEBPD is mainly involved in the regulation of genes related to immune and inflammatory responses [53]. SREBF1 has been associated with genes involved in sterol biosynthesis [54]. These genes are indeed hypo-methylated in the low-responder group and hyper-methylated in the high-responder group. However, the differences in baseline methylation status are not reflected in the baseline gene expression levels; these genes are expressed at baseline levels in both groups. In the AFB high-responder group, 4 genes show opposite $s$ of baseline methylation between the low and high responder group. POLR2K (full name: polymerase (RNA) II (DNA directed) polypeptide $K$ ) encodes for the smallest subunits of RNA polymerase II and is responsible for synthesizing mRNA [55]. TBL1X (full name: transducin (beta)-like 1X-linked) has been associate with the recruitment of the ubiquitin/19S proteasome-complex to nuclear receptor-regulated transcription units, where it acts in the activation of transcription [56]. SEC24A (full name: SEC24 family member A) is a component of coat protein II-coated vesicles, which promote the transport of plasma membrane, secretory, and vacuolar proteins from the endoplasmic reticulum to the golgi-complex [57]. ELOVL1 (full name: ELOVL fatty acid elongase 1) catalyzes the synthesis of monounsaturated and saturated very long chain fatty acids [58]. However, only half of the genes are indeed hypo-methylated at baseline for the high-responder group, while being hyper-methylated at baseline for the low-responder group. The other half of the genes is hyper-methylated at baseline in the highresponder group. Next, the baseline gene expression levels of gene $X$ and $Y$ were considered. Again, the differences in baseline methylation status of these genes were not reflected in a difference between responder groups in baseline gene expression of those genes. The genes were expressed at baseline level in each of the responder groups.

Overall, there does not seem to be any overwhelming evidence for a generalized effect of baseline methylation status of a gene on its DNA-damage response or its baseline gene expression status. Nevertheless, if the principle of separation on a response-group level is set aside Table 2 does show some genes for which the baseline methylation status is indicative for the baseline gene expression (highlighted in light gray, or striped). This led to the hypothesis that there might be genes for which the baseline interindividual variation in gene methylation level could explain the interindividual variation in 
baseline gene expression levels, and maybe even the variation between individuals in gene expression response to compound exposure. Indeed, with respect to the expression and activity of CYP enzymes, individual differences in DNA methylation status have often been suggested as causal factors for interindividual variation. [4, 59-63]. Also, the interindividual variation in CYP baseline gene expression levels has already been linked to the variation between individuals in gene expression response towards compound exposure in precision cut human liver slices [64]. Thus, genes with high interindividual variation in baseline methylation status and genes with high variability between individuals in baseline gene expression levels were defined.

Over-representation analysis on the genes with high interindividual variation in baseline methylation levels resulted in the identification of pathways mainly involved in 'neuronal systems' and 'signaling' (see Table 1, part B). An identical analysis on genes with high interindividual variation in baseline gene expression, defined a whole different set of pathways mainly related to 'metabolism' (also Table 1 part B). These results suggest interindividual variation in methylation affects a different set of genes than those that show a large interindividual variation in baseline gene expression levels. Nevertheless, for some genes our hypothesis (variation baseline methylation explains variation in baseline gene expression) seems still true.

By correlating the baseline gene methylation levels with the baseline gene expression levels of these genes using Spearman ranking of the donors, we sought for genes of which the baseline gene-expression could be explained by the baseline methylationstatus (see Figure 1). As could be expected from the results of the over-representation analysis, only a very limited set of genes shows a correlation between the variation over individuals in baseline gene methylation status expression and baseline gene expression levels. This again indicates that the baseline methylation-status of genes has only limited effects on baseline gene expression-levels. This conclusion is however, in line with the general opinion in current literature, that DNA methylation across individuals is not per se closely linked to gene expression [65-68].

Next, also the correlation analyses on the variation in baseline methylation status vs gene expression responses towards compound exposure, and variation in baseline gene expression levels vs response to compound exposure were added to the overlap-analysis to complete the picture (Figure 1), There does not seem to be a single gene for 
which a consistent pattern over donors in the correlation between variation in baseline methylation levels, in baseline gene expression and in gene expression response to carcinogens can be found. Thus, as observed o the group level, variation between baseline gene methylation status in combination with baseline gene expression levels is not indicative for the gene expression response towards compound exposure over individuals.

However, for 2 genes ( 1 for each compound) a partial overlap is present between the compound-induced gene expression response and baseline methylation levels or baseline gene expression levels. Correlations are depicted in Figure 2. The BMDs calculated from the compound-induced gene expression responses are the constant factor in these figures since the BMDs are always the same in correlation to baseline methylation level or baseline gene expression level. Thus for AFB (Figure 2A), the donors with a low BMD for WLS (full name Wntless Wnt ligand secretion mediator) are also the ones that have high baseline methylation levels for WLS according to the negative correlation between these parameters (depicted by triangles in opposite direction). Directed by a positive correlation, those donors with a low BMD for WLS are the same donors that have low baseline gene expression levels for WLS (depicted by a similar direction of triangles). Although no correlation between baseline methylation levels and baseline gene expression levels of WLS could be defined, those donors with a high baseline WLS methylation level are the ones that have a low baseline gene expression status for this gene (inverse directionality of triangles) as would be biologically plausible. Though there is no obvious link between WLS and AFB exposure available from literature, WLS is known to inhibit melanoma cell proliferation in human melanoma cell lines and induces spontaneous lung metastasis in xenografts in mice [69]. Due to the carcinogenic potency of AFB, a possible link between WLS and AFB can be imagined.

The overlapping gene related to the response induced by $\mathrm{BaP}$ was identified as LPXN (full name Leupaxin, see Figure 2B). Those donors with a low BMD for LPXN, are the same donors that have a low baseline methylation level for LPXN (directed by a positive correlation, depicted by a direction for the triangles). Also, the donors with a low BMD for LPXN are the same donors that have a high baseline gene expression status for LPXN (negative correlation, inverse direction of triangles). As with WLS/AFB, no correlation between baseline methylation status and baseline gene expression methylation levels of LPXN could be established. But again, the directionality of the triangles 
is opposite between baseline methylation status and baseline gene expression status. Thus donors with a low baseline LPXN methylation status also have a high level of LPXN baseline gene expression as would be biologically expected. An increase in LPXN gene-expression mediated through an aryl hydrocarbon receptor-related mechanism has previously been associated with exposure to BaP in mice [70]. In addition, LPXN expression was also affected in 2 human cell line models for human liver carcinoma exposed to $\mathrm{BaP}[34,71]$.

Some studies have proposed a non-continuous regulation of gene expression by DNA-methylation. In other words, the regulation of gene expression through methylation functions as an on/off-switch principle e.g. very low methylation (close to $0 \%$ ) versus very high methylation (close to 100\%) to allow or block transcription respectively [72-74]. If this is the case, it could at least partially explain the lack in established correlations in the analysis on the individual level, since the method behind this analysis assumes a gradient in the variation over individuals. The analysis on group level however does not include this assumption, and should therefore give better/more results if the above described situation is applicable. However, the results did not suggest so. Nevertheless, there are also studies suggesting more subtle changes in methylation status are having their effect on gene regulation like for instance methylation changes induced by environmental factors that are acquired over longer periods of time [75-78]. Thus, literature is still inconclusive on this subject.

In addition, recent literature suggests that the compounds used in this study (AFB and BaP) themselves might be able to influence methylation patterns during incubation with these compounds, also during short term exposure [79-82]. Changes in methylation during exposure which have consequences at the gene expression responselevel could be an additional factor explaining the low amount of correlations between baseline and response parameters detected in this study. The inclusion of an additional measure of methylation at the end of exposure to these compounds would thus be desirable.

Overall, literature on (baseline) methylation status was available only for a very limited number of genes detected in this study. This could be explained by the heavy bias towards aging, cancer (tumor vs. non-tumor) and immune-related research in methylation-related studies [10,11]. In general, baseline interindividual variation is not taken into consideration at all. Also, many of these studies consider only a limited set of genes, simply because of a lack of availability of genome- 
wide methylation assays as are used in the current study thereby possibly missing out on relevant genes. Finally, this study considers the interindividual variation in $\mathrm{PHH}$, an in vitro model for liver tissue. Due to the difficult access of the liver and thus limited availability of this tissue/cell-type it has not been a popular choice in previous methylation studies which tend to make use of blood as a reference tissue. Yet, methylation patterns have been shown to be highly tissue-specific and could thus very well be different in the before mentioned studies in comparison to the current, liver orientated study [83-85]. So, overall, knowledge on liver methylation patters is still limited. In addition, a standardization of the statistical approach, including the definition of relevant cut-off values for hyper/hypo-methylation levels and the size of the region in which methylation status is considered to affect a gene, would greatly increase the comparability of different studies.

The general conclusion from this study is that the interindividual variation in baseline methylation status only provides limited insight to explain interindividual variation in baseline gene expression levels and gene expression responses towards compound exposure. A general consensus concerning the biological mechanisms of gene expression regulation through gene methylation status is still far away and might be more complex in nature than previously appreciated. Although DNA-methylation status could still partially explain the observed interindividual variation in baseline and/or compound induced gene expression level, the inclusion of other (epi)genetic factors such histone modifications and microRNAs may lead to more insight in the intricate biological processes underlying interindividual variation.

\section{Acknowledgments}

This work was supported by CarcinoGENOMICS, a part of the $6^{\text {th }}$ framework project of the European Union aimed at developing in vitro methods for assessing the carcinogenic potential of compounds, as an alternative to current rodent bioassays for genotoxicity and carcinogenicity. 


\section{References}

1. Omiecinski, C.J., et al., Xenobiotic metabolism, disposition, and regulation by receptors: from biochemical phenomenon to predictors of major toxicities. Toxicol Sci, 2011. 120 Suppl 1: p. S49-75.

2. Urquhart, B.L., R.G. Tirona, and R.B. Kim, Nuclear receptors and the regulation of drug-metabolizing enzymes and drug transporters: implications for interindividual variability in response to drugs. J Clin Pharmacol, 2007. 47(5): p. 566-78.

3. Johansson, I. and M. Ingelman-Sundberg, Genetic Polymorphism and Toxicology-With Emphasis on Cytochrome P450. Toxicological Sciences, 2011. 120(1): p. 1-13.

4. Yang, L., et al., Gene expression variability in human hepatic drug metabolizing enzymes and transporters. PLoS One, 2013. 8(4): p. e60368.

5. Hernandez, A. and R. Marcos, Genetic variations associated with interindividual sensitivity in the response to arsenic exposure. Pharmacogenomics, 2008. 9(8): p. 1113-32.

6. Ketelslegers, H.B., et al., Multiplex Genotyping as a Biomarker for Susceptibility to Carcinogenic Exposure in the FLEHS Biomonitoring Study. Cancer Epidemiology Biomarkers \& Prevention, 2008. 17(8): p. 1902-1912.

7. Landi, S., Mammalian class theta GST and differential susceptibility to carcinogens: a review. Mutation research, 2000. 463(3): p. 247-83.

8. Godschalk, R.W., et al., Modulation of DNA and protein adducts in smokers by genetic polymorphisms in GSTM1,GSTT1, NAT1 and NAT2. Pharmacogenetics, 2001. 11(5): p. 389-98.

9. Hirvonen, A., Polymorphic NATs and cancer predisposition. IARC scientific publications, 1999(148): p. 251-70.

10. Fuso, A., The 'golden age' of DNA methylation in neurodegenerative diseases. Clinical chemistry and laboratory medicine : CCLM / FESCC, 2013. 51(3): p. 523-34.

11. Huidobro, C., A.F. Fernandez, and M.F. Fraga, The role of genetics in the establishment and maintenance of the epigenome. Cellular and molecular life sciences: CMLS, 2013. 70(9): p. 1543-73.

12. Eun, J.W., et al., Discriminating the molecular basis of hepatotoxicity using the large-scale characteristic molecular signatures of toxicants by expression profiling analysis. Toxicology, 2008. 249(2-3): p. 176-83.

13. Kohle, C., M. Schwarz, and K.W. Bock, Promotion of hepatocarcinogenesis in humans and animal models. Arch Toxicol, 2008. 82(9): p. 623-31.

14. Moller, P., Genotoxicity of environmental agents assessed by the alkaline comet assay. Basic Clin Pharmacol Toxicol, 2005. 96 Suppl 1: p. 1-42.

15. Morgan, E.T., et al., Regulation of drug-metabolizing enzymes and transporters in infection, inflammation, and cancer. Drug metabolism and disposition: the biological fate of chemicals, 2008. 36(2): p. 205-16.

16. Richardson, B.C., Role of DNA methylation in the regulation of cell function: autoimmunity, aging and cancer. The Journal of nutrition, 2002. 132(8 Suppl): p. 2401S-2405S.

17. Huidobro, C., et al., A DNA methylation signature associated with the epigenetic repression of glycine $\mathrm{N}$-methyltransferase in human hepatocellular carcinoma. Journal of molecular medicine, 2013. 91(8): p. 939-50.

18. Shitani, M., et al., Genome-wide analysis of DNA methylation identifies novel cancer-related genes in hepatocellular carcinoma. Tumour biology : the journal of the International Society for Oncodevelopmental Biology and Medicine, 
2012. 33(5): p. 1307-17.

19. Cotty, P.J., et al., Variation in polygalacturonase production among Aspergillus flavus isolates. Applied and environmental microbiology, 1990. 56(12): p. 3885-7.

20. Horn, B.W. and J.W. Dorner, Regional differences in production of aflatoxin B1 and cyclopiazonic acid by soil isolates of aspergillus flavus along a transect within the United States. Applied and environmental microbiology, 1999. 65(4): p. 1444-9.

21. Miller, J.A. and E.C. Miller, Natural and synthetic chemical carcinogens in the etiology of cancer. Cancer Res, 1965. 25(8): p. 1292-304.

22. Lawther, P.J. and R.E. Waller, Coal fires, industrial emissions and motor vehicles as sources of environmental carcinogens. IARC Sci Publ, 1976(13): p. 27-40.

23. Phillips, D.H., Polycyclic aromatic hydrocarbons in the diet. Mutat Res, 1999. 443(1-2): p. 139-47.

24. Beije, B. and L. Moller, 2-Nitrofluorene and related compounds: prevalence and biological effects. Mutat Res, 1988. 196(2): p. 177-209.

25. Zhou, S.F., J.P. Liu, and B. Chowbay, Polymorphism of human cytochrome P450 enzymes and its clinical impact. Drug Metab Rev, 2009. 41(2): p. 89-295.

26. Wild, C.P., et al., Aflatoxin B1 binding to plasma albumin and liver DNA upon chronic administration to rats. Carcinogenesis, 1986. 7(6): p. 853-8.

27. Invitrogen, No Standard Like a Gold Standard - InvitrogenTM Hepatic Biology Products. Invitrogen product brochure, 2009: p. 11-15.

28. Plazar, J., et al., Detection of xenobiotic-induced DNA damage by the comet assay applied to human and rat precision-cut liver slices. Toxicology in vitro an international journal published in association with BIBRA, 2007. 21(6): p. 1134-1142.

29. Wilkening, S., F. Stahl, and A. Bader, Comparison of primary human hepatocytes and hepatoma cell line Hepg2 with regard to their biotransformation properties. Drug metabolism and disposition: the biological fate of chemicals, 2003. 31(8): p. 1035-42.

30. Li, A.P., Metabolism Comparative Cytotoxicity Assay (MCCA) and Cytotoxic Metabolic Pathway Identification Assay (CMPIA) with cryopreserved human hepatocytes for the evaluation of metabolism-based cytotoxicity in vitro: proofof-concept study with aflatoxin B1. Chem Biol Interact, 2009. 179(1): p. 4-8.

31. Langouet, S., et al., Metabolism of aflatoxin B1 by human hepatocytes in primary culture. Adv Exp Med Biol, 1996. 387: p. 439-42.

32. Jetten, M.J., et al., Baseline and genotoxic compound induced gene expression profiles in HepG2 and HepaRG compared to primary human hepatocytes. Toxicology in vitro : an international journal published in association with BIBRA, 2013. 27(7): p. 2031-40.

33. Rothkamm, K. and M. Lobrich, Evidence for a lack of DNA double-strand break repair in human cells exposed to very low $\mathrm{x}$-ray doses. Proceedings of the National Academy of Sciences of the United States of America, 2003. 100(9): p. 5057-62.

34. Jennen, D.G., et al., Comparison of HepG2 and HepaRG by whole-genome gene expression analysis for the purpose of chemical hazard identification. Toxicol Sci, 2010. 115(1): p. 66-79.

35. Eijssen, L.M., et al., User-friendly solutions for microarray quality control and pre-processing on ArrayAnalysis.org. Nucleic acids research, 2013. 41(Web Server issue): $p$. W71-6.

36. Irizarry, R.A., et al., Exploration, normalization, and summaries of high density oligonucleotide array probe level data. Biostatistics, 2003. 4(2): p. 249-64. 
37. University of Michigan. Brainarray custom CDF. Version 16.0.0; Available from: http://brainarray.mbni.med.umich.edu/Brainarray/Database/CustomCDF/ cdfreadme.htm.

38. Slob, W., et al., A statistical evaluation of toxicity study designs for the estimation of the benchmark dose in continuous endpoints. Toxicol Sci, 2005. 84(1): p. 167-85.

39. Lisanti, S., T. von Zglinicki, and J.C. Mathers, Standardization and quality controls for the methylated DNA immunoprecipitation technique. Epigenetics : official journal of the DNA Methylation Society, 2012. 7(6): p. 615-25.

40. Weber, M., et al., Chromosome-wide and promoter-specific analyses identify sites of differential DNA methylation in normal and transformed human cells. Nature genetics, 2005. 37(8): p. 853-62.

41. Weber, M. and D. Schubeler, Genomic patterns of DNA methylation: targets and function of an epigenetic mark. Current opinion in cell biology, 2007. 19(3): p. 273-80.

42. Kamburov, A., et al., The ConsensusPathDB interaction database: 2013 update. Nucleic acids research, 2013. 41(Database issue): p. D793-800.

43. Kyrtopoulos, S.A., Variability in DNA repair and individual susceptibility to genotoxins. Clin Chem, 1995. 41(12 Pt 2): p. 1848-53.

44. Singh, N. and T.A. Venkitasubramanian, Effect of aflatoxin B1 on lipids of rat tissues. Environ Physiol Biochem, 1975. 5(3): p. 147-57.

45. Shen, H.M., C.N. Ong, and C.Y. Shi, Involvement of reactive oxygen species in aflatoxin B1-induced cell injury in cultured rat hepatocytes. Toxicology, 1995. 99(1-2): p. 115-23.

46. Jungnickel, P.W., K.A. Cantral, and P.A. Maloley, Pravastatin: a new drug for the treatment of hypercholesterolemia. Clin Pharm, 1992. 11(8): p. 677-89.

47. McKenney, J.M., Lovastatin: a new cholesterol-lowering agent. Clin Pharm, 1988. 7(1): p. 21-36.

48. Zhou, S.F., et al., Structure, function, regulation and polymorphism and the clinical significance of human cytochrome P450 1A2. Drug Metab Rev, 2010. 42(2): p. 268-354.

49. Crespi, C.L., et al., Development of a human lymphoblastoid cell line constitutively expressing human CYP1B1 cDNA: substrate specificity with model substrates and promutagens. Mutagenesis, 1997. 12(2): p. 83-9.

50. Denison, M.S., et al., Ligand binding and activation of the Ah receptor. Chem Biol Interact, 2002. 141(1-2): p. 3-24.

51. Branzei, D. and M. Foiani, Regulation of DNA repair throughout the cell cycle. Nature reviews. Molecular cell biology, 2008. 9(4): p. 297-308.

52. Jiang, Y., et al., BaP-induced DNA damage initiated p53-independent necroptosis via the mitochondrial pathway involving Bax and $\mathrm{BCl}-2$. Human \& Experimental Toxicology, 2013. 32(12): p. 1245-1257.

53. Newton, K. and V.M. Dixit, Signaling in innate immunity and inflammation. Cold Spring Harb Perspect Biol, 2012. 4(3).

54. Georgiadi, A. and S. Kersten, Mechanisms of gene regulation by fatty acids. Adv Nutr, 2012. 3(2): p. 127-34.

55. Takahashi, H., et al., Human mediator subunit MED26 functions as a docking site for transcription elongation factors. Cell, 2011. 146(1): p. 92-104.

56. Perissi, V., et al., A corepressor/coactivator exchange complex required for transcriptional activation by nuclear receptors and other regulated transcription factors. Cell, 2004. 116(4): p. 511-26.

57. Pagano, A., et al., Sec24 proteins and sorting at the endoplasmic reticulum. J Biol Chem, 1999. 274(12): p. 7833-40. 
58. Kihara, A., Very long-chain fatty acids: elongation, physiology and related disorders. J Biochem, 2012. 152(5): p. 387-95.

59. Ivanov, M., M. Kacevska, and M. Ingelman-Sundberg, Epigenomics and interindividual differences in drug response. Clinical pharmacology and therapeutics, 2012. 92(6): p. 727-36.

60. Kacevska, M., M. Ivanov, and M. Ingelman-Sundberg, Perspectives on epigenetics and its relevance to adverse drug reactions. Clinical pharmacology and therapeutics, 2011. 89(6): p. 902-7.

61. Kacevska, M., M. Ivanov, and M. Ingelman-Sundberg, Epigenetic-dependent regulation of drug transport and metabolism: an update. Pharmacogenomics, 2012. 13(12): p. 1373-85.

62. Hirota, T., et al., Epigenetic regulation of genes encoding drug-metabolizing enzymes and transporters; DNA methylation and other mechanisms. Current drug metabolism, 2008. 9(1): p. 34-8.

63. Ivanov, M., et al., In-solution hybrid capture of bisulfite-converted DNA for targeted bisulfite sequencing of 174 ADME genes. Nucleic acids research, 2013. 41(6): p. e72.

64. Jetten, M.J., et al., Interindividual variation in response to xenobiotic exposure established in precision-cut human liver slices. Toxicology, 2014. 323C: p. 61-69.

65. Lam, L.L., et al., Factors underlying variable DNA methylation in a human community cohort. Proceedings of the National Academy of Sciences of the United States of America, 2012. 109 Suppl 2: p. 17253-60.

66. Gibbs, J.R., et al., Abundant quantitative trait loci exist for DNA methylation and gene expression in human brain. PLoS Genet, 2010. 6(5): p. e1000952.

67. Bell, J.T., et al., DNA methylation patterns associate with genetic and gene expression variation in HapMap cell lines. Genome Biol, 2011. 12(1): p. R10.

68. Relton, C.L., et al., DNA methylation patterns in cord blood DNA and body size in childhood. PLoS One, 2012. 7(3): p. e31821.

69. Yang, P.T., et al., WLS inhibits melanoma cell proliferation through the betacatenin signalling pathway and induces spontaneous metastasis. EMBO Mol Med, 2012. 4(12): p. 1294-307.

70. Kerley-Hamilton, J.S., et al., Inherent and benzo[a]pyrene-induced differential aryl hydrocarbon receptor signaling greatly affects life span, atherosclerosis, cardiac gene expression, and body and heart growth in mice. Toxicol Sci, 2012. 126(2): p. 391-404.

71. Magkoufopoulou, C., et al., Comparison of phenotypic and transcriptomic effects of false-positive genotoxins, true genotoxins and non-genotoxins using HepG2 cells. Mutagenesis, 2011. 26(5): p. 593-604.

72. Brunner, A.L., et al., Distinct DNA methylation patterns characterize differentiated human embryonic stem cells and developing human fetal liver. Genome Res, 2009. 19(6): p. 1044-56.

73. Meissner, A., et al., Genome-scale DNA methylation maps of pluripotent and differentiated cells. Nature, 2008. 454(7205): p. 766-70.

74. Xie, H., et al., Genome-wide quantitative assessment of variation in DNA methylation patterns. Nucleic Acids Res, 2011. 39(10): p. 4099-108.

75. Bock, C., et al., Inter-individual variation of DNA methylation and its implications for large-scale epigenome mapping. Nucleic acids research, 2008. 36(10): p. e55.

76. Hammons, G.J., et al., Specific site methylation in the $5^{\prime}$-flanking region of CYP1A2 interindividual differences in human livers. Life Sci, 2001. 69(7): p. 839-45. 
77. Sandovici, I., et al., Interindividual variability and parent of origin DNA methylation differences at specific human Alu elements. Human molecular genetics, 2005. 14(15): p. 2135-43.

78. Yasar, U., et al., Evidence for regulation of UDP-glucuronosyltransferase (UGT) $1 \mathrm{~A} 1$ protein expression and activity via DNA methylation in healthy human livers. The Journal of pharmacy and pharmacology, 2013. 65(6): p. 874-83.

79. Corrales, J., et al., Effects on specific promoter DNA methylation in zebrafish embryos and larvae following benzo[a]pyrene exposure. Comp Biochem Physiol C Toxicol Pharmacol, 2014. 163: p. 37-46.

80. Fang, $X$., et al., Benzo[a]pyrene decreases global and gene specific DNA methylation during zebrafish development. Environ Toxicol Pharmacol, 2013. 36(1): p. 40-50.

81. Wu, H.C., et al., Global DNA methylation in a population with aflatoxin B1 exposure. Epigenetics : official journal of the DNA Methylation Society, 2013. 8(9): p. 962-9.

82. Zhu, C.C., et al., Effect of Mycotoxin-Containing Diets on Epigenetic Modifications of Mouse Oocytes by Fluorescence Microscopy Analysis. Microsc Microanal, 2014: p. 1-9.

83. Bird, A., DNA methylation patterns and epigenetic memory. Genes \& development, 2002. 16(1): p. 6-21.

84. Jaenisch, R. and A. Bird, Epigenetic regulation of gene expression: how the genome integrates intrinsic and environmental signals. Nature genetics, 2003. 33 Suppl: p. 245-54.

85. Byun, H.M., et al., Epigenetic profiling of somatic tissues from human autopsy specimens identifies tissue- and individual-specific DNA methylation patterns. Human molecular genetics, 2009. 18(24): p. 4808-17. 


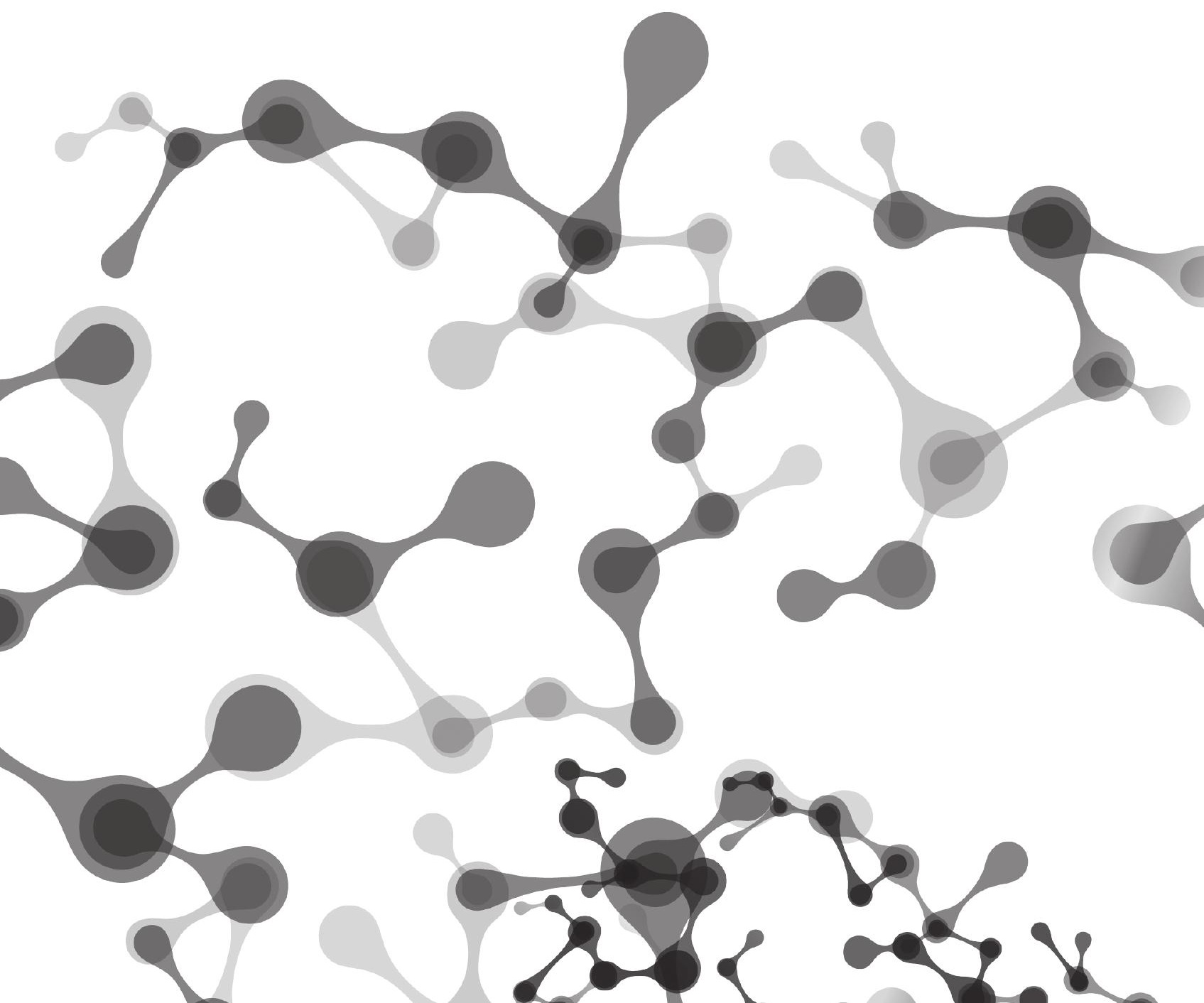




\section{Chapter 6 \\ 'Omics analysis of low dose acetaminophen intake demonstrates novel response pathways in humans}

Marlon J.A. Jetten

Stan Gaj

Ainhoa Ruiz-Aracama

Theo M.C.M. de Kok Joost H.M. van Delft

Arjen Lommen

Eugene P. van Someren

Danyel G.J. Jennen

Sandra M. Claessen

Ad A.C.M. Peijnenburg

Rob H. Stierum

Jos C.S. Kleinjans

Published: Toxicology and Applied Pharmacology: Volume 259, Issue 3,

15 March 2012, Pages 320-328 


\section{Abstract}

Acetaminophen is the primary cause of acute liver toxicity in Europe/USA, which led the FDA to reconsider recommendations concerning safe acetaminophen dosage/use. Unfortunately, the current tests for liver toxicity are no ideal predictive markers for liver injury, i.e. they only measure acetaminophen exposure after profound liver toxicity has already occurred. Furthermore, these tests do not provide mechanistic information. Here, 'omics techniques (global analysis of metabolomic/gene-expression responses) may provide additional insight.

To better understand acetaminophen-induced responses at low doses, we evaluated the effects of (sub-)therapeutic acetaminophen doses on metabolite formation and global gene-expression changes (including, for the first time, full-genome human miRNA expression changes) in blood/urine samples from healthy human volunteers.

Many known and several new acetaminophen-metabolites were detected, in particular in relation to hepatotoxicity-linked, oxidative metabolism of acetaminophen. Transcriptomic changes indicated immune-modulating effects ( $2 \mathrm{~g}$ dose) and oxidative stress responses ( $4 \mathrm{~g}$ dose). For the first time, effects of acetaminophen on full-genome human miRNA expression have been considered and confirmed the findings on mRNA level.

'Omics techniques outperformed clinical chemistry tests and revealed novel response pathways to acetaminophen in humans. Although no definitive conclusion about potential immunotoxic effects of acetaminophen can be drawn from this study, there are clear indications that the immune system is triggered even after intake of low doses of acetaminophen. Also, oxidative stress-related gene responses, similar to those seen after high dose acetaminophen exposure, suggest the occurrence of possible pre-toxic effects of therapeutic acetaminophen doses. Possibly, these effects are related to dosedependant increases in levels of hepatotoxicity-related metabolites. 


\section{Introduction}

An extensively studied hepatotoxicant in humans is acetaminophen (APAP), a readily available over-the-counter drug of which the exact pharmacological mode of action is still partially unknown. APAP is the most common cause of acute liver toxicity in Europe and the US $[1,2]$. Therefore, the FDA recently reconsidered recommendations concerning the single adult APAP dose and the maximum total dose for $24 \mathrm{~h}$. Also, packaging and labeling regulations were changed and elimination of prescription APAP combination products has been recommended [3-5].

Diagnosis of liver toxicity is based on evaluating liver function through serum parameters such as alanine aminotransferase (ALT). Unfortunately, none of these tests represent ideal biomarkers since they are positive only when pronounced liver injury has already occurred [6, 7]. This implies that these assays cannot predict the onset or severity of liver injury. In this respect, the more sensitive 'omics techniques (analysis of genomic and metabolomic responses to chemical challenges) may contribute [8-11]. Significant gene expression changes in rat blood cells upon administration of APAP were detected well below liver damage levels as diagnosed by classical parameters [12]. The additional advantage of these technologies is their ability to study changes at a molecular level. This provides additional information on compoundspecific modes-of-action which may precede classic, often toxicological endpoints [13].

Rodent-based data on in vivo metabolomic/transcriptomic responses in liver [14-19], plasma [14, 18], blood [12], serum [20, 21] and/or urine [21] after APAP exposure are abundantly available. Data on transcriptomic responses to low, therapeutic APAP doses in humans however are scarce. The transcriptome responses in lymphocytes from a few human APAP suicide cases, which provide insight in overtly toxic responses, are similar to those described in animal studies [12]. Fannin et al. [22] showed that in humans a $4 \mathrm{~g}$ single dose of APAP caused changes in the transcriptome of peripheral blood cells, mainly involving the down-regulation of oxidative phosphorylation genes. These gene expression changes are similar to those seen after high doses of APAP. At these high doses these responses are associated with APAP-induced toxicity and liver injury. However, in the study of Fannin et al. overt hepatotoxicity did not occur. In addition, it is of interest to note that, although the liver is the primary site for APAP toxicity, the blood gene expression profile can actually be used as a surrogate to monitor liver 
function/toxicity upon APAP administration [16, 22-24] and even APAP dose levels [12].

In addition, changes in microRNA (miRNA) expression profiles in plasma and/or liver after high dose APAP exposure were recently investigated in 2 rodent studies and 1 human study [25-27]. In rodents, changes in miRNA expression profiles were observed hours before any other toxic effects on morphology, or changes in ALT or lactate levels could be detected. To our knowledge, no data on full-genome human miRNA expression responses after APAP intake are currently available.

In view of the FDA's considerations mentioned above, we aimed at exploring the effects of low APAP doses in humans. We hypothesized that blood transcriptomics (both mRNA and miRNA analysis) and blood/ urine metabolomics are sufficiently sensitive and robust for this purpose. Furthermore, this is the first study which considers human full-genome miRNA expression-profile changes as possible indicators of APAPinduced molecular effects.

\section{Materials and methods}

\section{Human subjects}

The experimental protocol was approved by the Medical Ethics Committee of the University Hospital Maastricht and all participants signed an informed consent. Subjects were healthy, aged $48.4^{+} \% 4.5,5$ males and 2 females, 1 being a smoker. Two weeks prior and during the intervention subjects did not take any additional medication.

\section{Study design and sample collection}

Subjects took $0.5,2$ or $4 \mathrm{~g}$ of APAP over $24 \mathrm{~h}$ in the form of crushed 500mg APAP tablets (Healthypharm) through oral self administration; daily doses were spread over equal amounts and taken at $6 \mathrm{~h}$ intervals. Interference due to repeated APAP exposure was prevented by a wash-out period of at least 2 weeks between experimental rounds. All subjects successfully completed the study, with the exception of one subject (a 49 year old non-smoking female), who participated in the $0.5 \mathrm{~g}$ and $2 \mathrm{~g}$ dose-round and was replaced by another subject (41 year old non-smoking male) in the $4 \mathrm{~g}$ dose-round. Sampling was performed as indicated in Figure 1. The time at which experimental rounds were started were kept constand to minimize the effects of diurnal variation between rounds. 


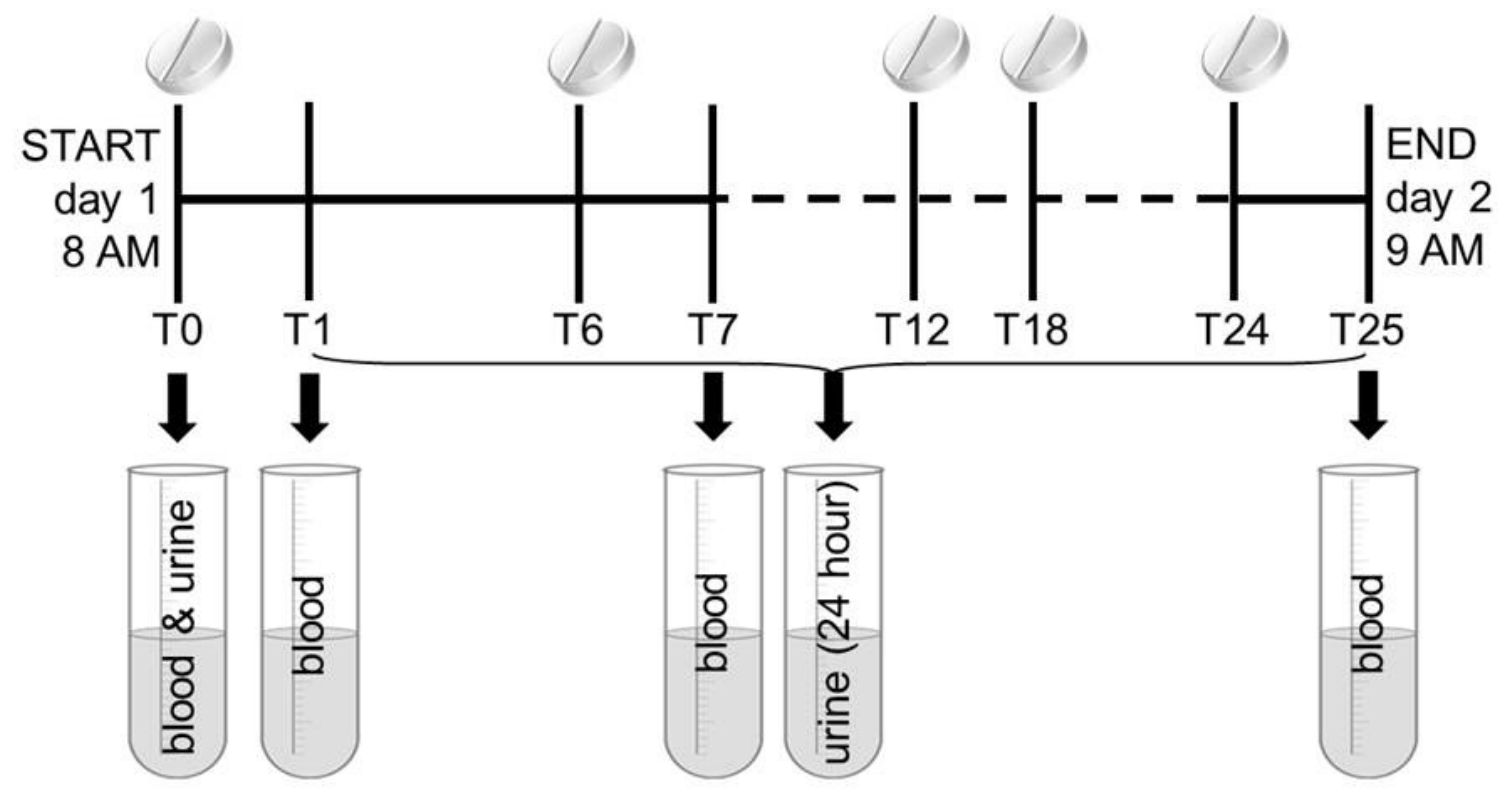

Figure 1; Sampling and dosing schedule

Pills indicate APAP dosing time points, tubes indicate sampling of blood and/or urine at the corresponding time point ( $T$ in hours) for each dose round. In the $4 g$ APAP dose round, blood samples for transcriptomic analyses were not collected on T1 and T7.

\section{Clinical chemistry}

Plasma from each donor collected before (T0) and after (T25) APAP intake was analyzed for; ALT, total bilirubin, alkaline phosphatase, gamma glutamyl transpeptidase, lactate dehydrogenase and albumin using standard clinical liver chemistry tests.

\section{Metabolomic analyses}

For analysis of serum and urine samples, a combination of NMR, LC-MS and/or GC-MS has been used [28].

Urine

A mixture of urine:acetone (1:4) was prepared (3-fold). Samples were centrifuged; supernatant was collected, dried under nitrogen and reconstituted $(30 \mathrm{mg} / \mathrm{ml})$ in $99.95 \%$ D2O. Urinary excretion of APAP and its metabolites was normalized against measured urinary concentrations of androstanetriol/androstan-diol-one-glucoronide, etiocholanoneglucoronide and androstenetriol-glucoronide which are expected to be constant. Part of the sample was stored for LC-MS analysis. To the 
remainder $1 \mathrm{M}$ phosphate buffer (90:10; v:v; $\mathrm{pH}=7)$ in $99.95 \%$ D2O was added for NMR analysis.

Serum

From serum of coagulated blood (30min., room temperature) a polar and an apolar metabolite fractions was extracted as follows:

- $\quad$ Polar fraction: Preparation is identical to the procedure as described for urine samples (see above), with the exception that the reconstituted sample was extracted with deuterated chloroform to obtain a lipid free sample. From the resulting D2O fraction a part was kept for LC-MS analysis. The NMR sample was prepared as with urine.

- $\quad$ Apolar fraction: To $1 \mathrm{ml}$ of serum, 10ml of CD3OD:CDCl3 in a proportion 2:1 (v:v) was added and centrifuged. The chloroformphase was collected, dried under nitrogen, reconstituted in $1 \mathrm{ml}$ of deuterated chloroform and re-extracted with $99.95 \%$ D2O. A fraction of this sample was used for NMR analysis and the remainder was stored for GC-MS analysis after derivatization.

Data analyses/metabolite identification

NMR data were pre-processed and aligned using an in-house developed program [29]. GC-MS and LC-MS data were pre-processed and aligned by means of metAlign [30].

- $\quad$ Untargeted univariate analyses: A combination of high fold change (FC) and relatively strict $p$-values was used ( $F C \geq 2-10$, $p<0.01$ ) was used to exclude selection of false positives from the differential data sets due to the high inter-individual variation and a limited number of samples, . Signals complying with these conditions were further identified based on literature $[21,31]$. Statistically significant masses selected from LCMS analysis were identified by means of LC-nanomate-Orbitrap for elemental composition analysis of $\mathrm{MS}^{\mathrm{n}}$ fragments $[32,33]$.

- $\quad$ Targeted analyses: A list of masses of interest was loaded into Search LCMS [28] to create a file containing the amplitudes of these masses.

Metabolite visualisation

Pathvisio, a pathway visualization tool, was used to create a metabolic map [34]. This metabolic map was based on available literature [21,35]. LC-MS data were averaged per group level $(n=6)$ and 
visualized per test fluid (polar serum fraction and urine), time point and dose. For visualization purposes only, all 0 values were manually set to 8 (which is almost equal to the lowest measured value). Then al values were log-transformed resulting in a data range with a minimum of 3 and a maximum of 17 .

\section{Transcriptomic analyses}

mRNA/miRNA isolation

Total RNA was isolated from whole blood (PAXgene Blood RNA kit, PreAnalytiX/Qiagen), treated with DNase and globin reduced (GLOBINclear kit, Ambion) according to the manufacturer's protocols. Total RNA for miRNA analyses was isolated using the RiboPure-Blood kit (Ambion) according to the manufacturer's protocol which included a DNase digestion. Integrity of all RNA was checked on a Bioanalyzer (Agilent).

mRNA/miRNA microarray analyses

All data are MIAME compliant and the raw micro-array data have been deposited in the GEO database (GSE30418 - Low dose human APAP exposure).

- $\quad$ mRNA: cRNA targets were prepared according to the Affymetrix protocol for an input of 250ng RNA. cRNA was hybridized (Affymetrix Human Whole Genome U133A plus 2 GeneChip) according to the manufacturer's recommended procedures. After hybridization GeneChips were washed/stained using a fluidics station (Affymetrix) and scanned (GeneArray scanner, Affymetrix).

- $\quad$ miRNA: $1 \mu \mathrm{g}$ of total blood cell RNA was labeled (miRCURY_LNA microRNA-array kit_V11.0, Exiqon) according to the manufacturer's protocol (single color labeling) and scanned (Genepix_4000B scanner with software version 3.0 (Axon)). Expression of miR-19a, miR-19b and miR-374a was confirmed with RT-PCR (TaqMan MicroRNA Assays, Applied Biosystems) according to the manufacturer's protocol. FC's were calculated according to the $\triangle \triangle$ Ct-method, using U6 snRNA for normalization [36]. The miRNA array of one donor (subject E) was aberrant in intensities from all others and was therefore excluded from further analyses. 
mRNA-array statistical analyses

After quality control, data were re-annotated (EntrezGene customCDF_V13) $[37,38]$. Next, the data were RMA normalized and filtered for expressed genes using present/absent-calls obtained from the MAS5 algorithm; only data with less than 2 absent calls/reporter over the 6 arrays/time point were included. In the $4 \mathrm{~g}$ APAP dose round, blood sampling was not preformed on T1 and T7 for mRNA analyses, instead additional samples on T0 and T25 were collected to enable miRNA analyses as will be described below. Significant Analyses of Micro-arrays (SAM) was performed to find differentially expressed genes (DEGs) $[39,40]$. SAM analyses was performed per time point (two class paired analyses with $F C \geq 1.5$ and a false discovery rate $(F D R) \leq 0.1$, for $0.5 \mathrm{~g}$ and $2 \mathrm{~g}$ at T1, T7 and T25 for $4 \mathrm{~g}$ at T25 only). In addition, an analysis over time (Time Course, TC) was performed (one class time course, FDR $\leq 0.1$, for $0.5 \mathrm{~g}$ and $2 \mathrm{~g}$ only). Finally, significantly altered biological pathways were identified using MetaCore (GeneGO, $\mathrm{p}<0.05$, only pathways with at least 5 gene products were taken into consideration). Pathway analyses appoints individual genes to pathways consisting of biologically related genes representing a certain biological function/effect, which not only enhances statistical power, but also enables easier interpretation of gene-expression data at a higher hierarchical level of cellular function.

\section{miRNA-array statistical analyses}

Genepix data files were imported into the statistical program $\mathrm{R}$ [41]. After a thorough quality control all datapoints were backgroundcorrected, log-transformed, filtered for human miRNAs and quantile normalized (after 4g T25 only). MiRNAs have 4 technical replicates/chip, when at least 3 out of 4 possible signals were present, mean expression values were calculated. Only miRNAs with $\mathrm{p}<0.05$ (t-test) and $\mathrm{FC} \geq 1.5$ were considered to be differentially expressed. For these miRNAs, a target scan was performed (Microcosm) to identify target genes. Expression of mRNA products of these target genes (target mRNA) was checked by linking to the corresponding mRNA arrays (4g at T25). Only target genes of which the mRNA product was significantly differentially expressed in the opposite direction of the miRNA were taken into consideration. These target genes were arranged into categories according to their involvement in cell processes (taken from GeneCardsV3, similar to mRNA analyses). 


\section{Results}

\section{Clinical chemistry}

Averaged data from all individuals $(n=6)$ showed no response towards liver toxicity in any of the clinical liver tests (student's t-test T0 vs. T25, all P>0.05, raw data available in Supplementary Table A).

\section{Metabolomics}

Urine

A total of 23 masses could be identified; all were derived from APAP (Supplementary Table B). Several metabolites could be detected at all doses; APAP as mother compound, APAP-glucuronide, APAP-sulfate, cysteine-APAP and N-acetylcysteine-APAP (Supplementary Table B). The latter 2 compounds are derivatives of NAPQI, which is thought to cause the toxic effects of high APAP doses through protein-adduct formation leading to oxidative stress and finally liver damage [42]. There was no statistical evidence for secondary (non-APAP) changes in urine.

Serum

- $\quad$ Polar metabolite fraction: The serum dataset showed less significant differences than urine samples (Supplementary Table B). As in urine, all masses were derived from APAP, but their levels were much lower. Again, there was no evidence for secondary changes.

- $\quad$ Apolar metabolite fraction: Statistical analysis did not show significant differences in chemical profiles upon APAP treatment.

Pathway visualization

Data from urine and polar serum metabolites were visualized in a metabolic pathway (Figure 2, based on raw data available in Supplementary Table B). Five of these APAP metabolites, 3 glucuronide products, an APAP-sulphate conjugate and what seems to be an APAPtryptophan product, have never been described before (Supplementary Table B, highlighted in gray). APAP itself and APAP-glucuronide are most strongly elevated. In general, a dose-dependent and time-dependent effect is apparent, especially in serum. With an increase in APAP-dose, relatively more cysteine-, hydroxy- and methoxy-metabolites were formed. 
APAP.

( $<5 \%$ Renal excretion)

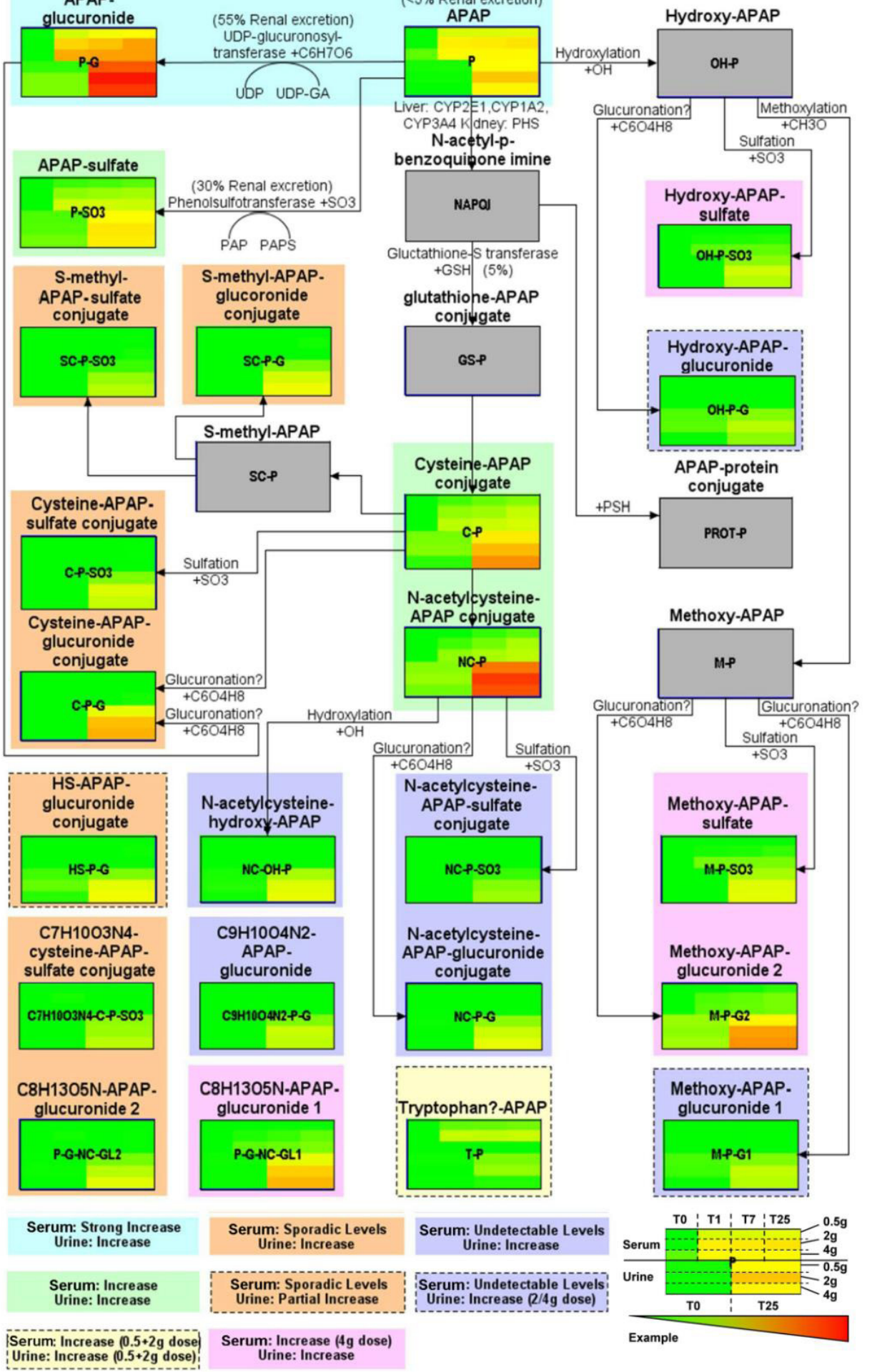


Figure 2; Schematic visualization of APAP metabolic pathway

The group averages $(n=6)$ for each metabolite per dose, time point and sample material are visualized as in the example. Gray boxes; not measured/detected. Increase in a metabolite is pictured from green (no increase, equals a numerical value of 3 on a log-scale) to yellow, orange and red (high increase, maximum value equals 17 on a log-scale). Raw data are available in supplementary table $B$.

\section{Transcriptomics}

mRNA expression

DEGs: After intake of $4 \mathrm{~g}$ of APAP (T25 time-point was analyzed only) transcriptomics analyses resulted in $285 \mathrm{DEGs}$ (FDR=0.004). At lower APAP doses. Intermediate time points were also considered. Intake of $2 \mathrm{~g}$ of APAP resulted in DEGs at T1 (16, FDR=0.095), T7 (2, $\mathrm{FDR}=0.000), \mathrm{T} 25$ (254, FDR=0.016) and over time (3484, FDR=0.094). After intake of $0.5 \mathrm{~g}$ of APAP, DEGs were detected at T25 (7, FDR=0.056) and over time (2, FDR=0.000) only. In general, almost all genes regulated at a specific time point were also affected over time.

Pathway analyses: 8 pathways were regulated at T25 after $4 \mathrm{~g}$ of APAP (see Supplementary Table C). Intake of $2 \mathrm{~g}$ of APAP resulted in 14, 3, 18 and 15 pathways to be significantly affected at T1, T7, T25 and over time (TC) respectively. No biological pathways were significantly regulated at any specific time point or over time after $0.5 \mathrm{~g}$ of APAP.

Cell processes: Each significant pathway was categorized according to the cell process to which it relates (Supplementary Table $C$, third column). Table 1 shows the percentage of affected pathways over total pathways at that particular dose/time point. After intake of $2 \mathrm{~g}$ of APAP, mainly immune response pathways were regulated, including "macrophage inhibitory factor mediated glucocorticoid regulation", "signaling of several interleukins, CD40" and "T-helper17 cell related processes". This response changed after intake of $4 \mathrm{~g}$ of APAP towards pathways involved in "apoptosis and survival/oxidative stress/DNA damage" (mainly oxidative phosphorylation) and "cholesterol transport" (mainly cholesterol and Sphingolipids transport). The category "other processes" in Table 1 contains several pathways which could not be scored within any of the other categories. these pathways mainly represent very general (blood) cell processes. 
Table 1; Cell processes affected after intake of $2 g$ and $4 g$ of APAP

MetaCore pathways that were significantly affected were counted and divided over general cell

processes. For each cell process, the number of corresponding effected pathways was scored as \%

\begin{tabular}{|c|c|c|c|c|c|}
\hline \multirow[b]{2}{*}{ Cell process } & \multicolumn{5}{|c|}{ APAP dose/ time point } \\
\hline & $2 g / T 1$ & $2 g / T 7$ & $2 g / T 25$ & $\begin{array}{l}2 \mathrm{~g} / \mathrm{Time} \\
\text { Course }\end{array}$ & $4 \mathrm{~g} / \mathrm{T} 25$ \\
\hline Immune response & 29 & 0 & 56 & 33 & 13 \\
\hline Lipid metabolism & 7 & 0 & 6 & 0 & 13 \\
\hline Cholesterol transport & 0 & 0 & 11 & 0 & 25 \\
\hline $\begin{array}{l}\text { Apoptosis and survival/oxida- } \\
\text { tive stress/DNA damage }\end{array}$ & 0 & 0 & 11 & 20 & 25 \\
\hline Drug metabolism/pain & 36 & 0 & 0 & 7 & 0 \\
\hline Other processes & 29 & 100 & 17 & 40 & 25 \\
\hline
\end{tabular}

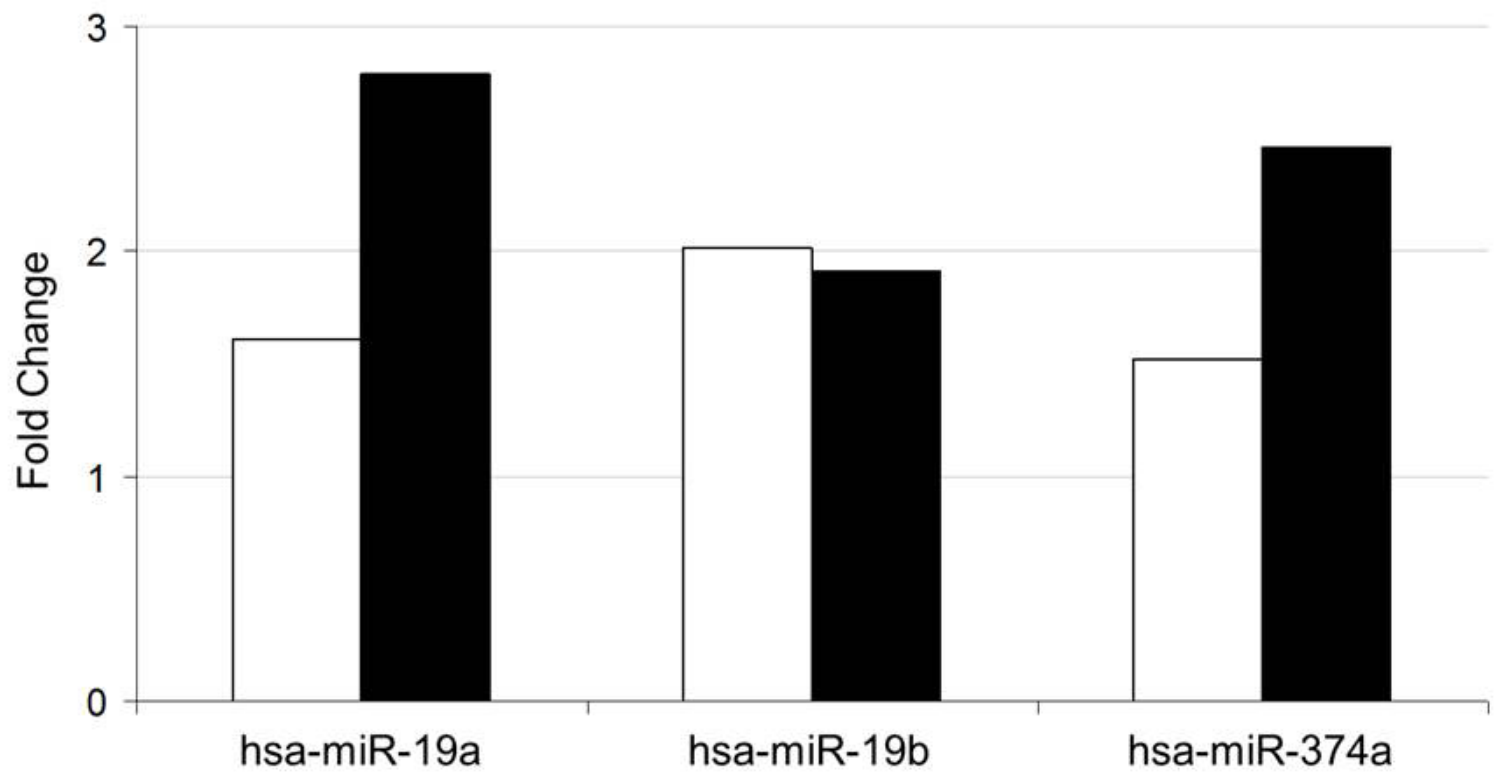

Figure 3; Confirmation of miRNA expression

Fold changes of miRNA expression (TO vs. T24) according to microarray analyses (white bars) were confirmed by RT-PCR (black bars). 


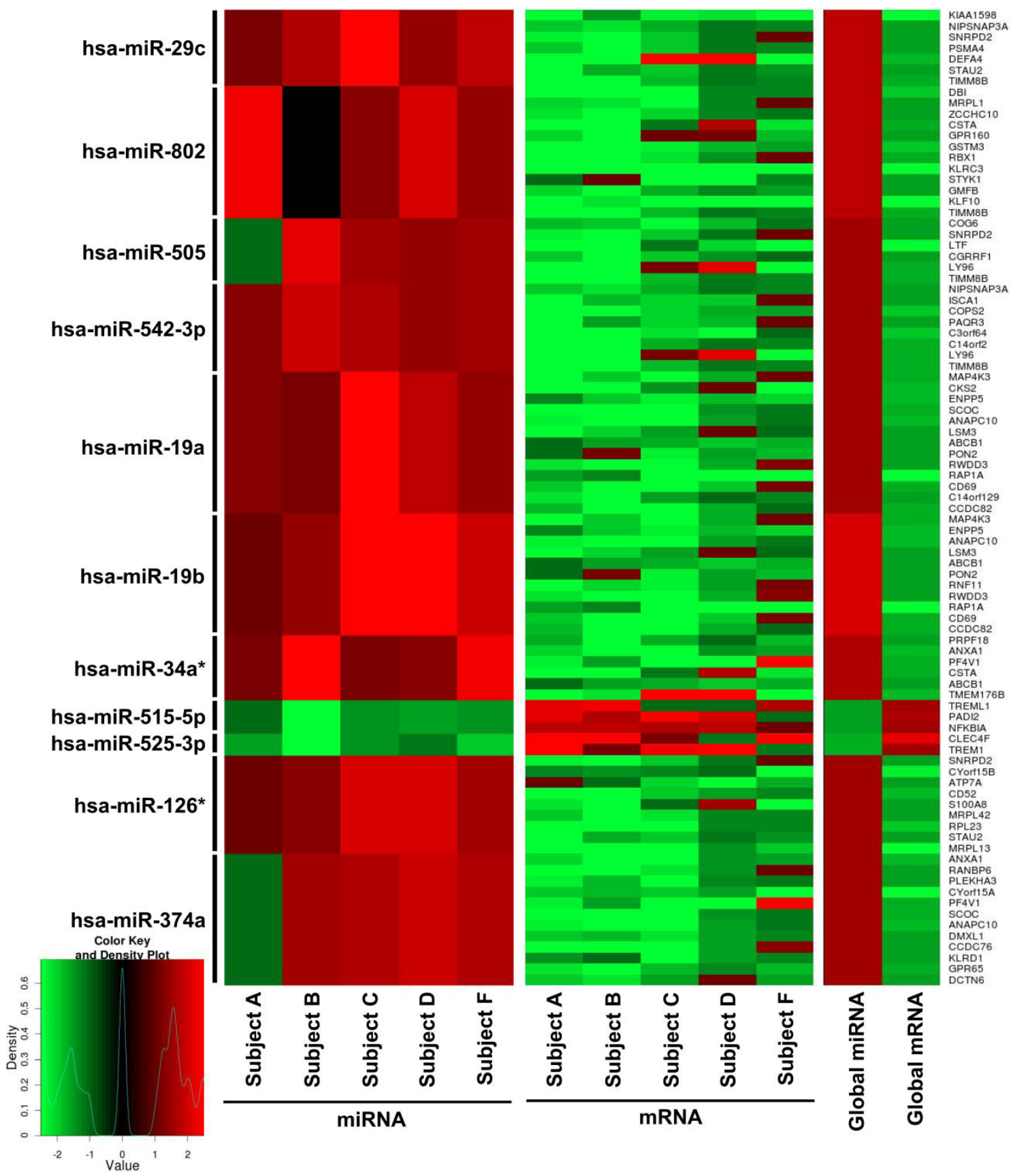

Figure 4; Fold changes of miRNAs and their negatively correlated mRNA targets From left to right: miRNAs, individual miRNA expression (FC) per subject, individual target mRNA expression (FC) per subject, group average (global) miRNA/target mRNA expression and target $m R N A$ gene name. Red and green mean up and down regulation respectively after intake of $4 \mathrm{~g}$ APAP over 24 hours. 


\section{miRNA expression}

Differentially expressed miRNAs: Blood miRNA levels were determined after intake of $4 \mathrm{~g}$ of APAP (T25); 11 miRNAs were significantly regulated. Differential expression of 3 miRNAs was confirmed by means of RT-PCR (Figure 3). 89 mRNA products of predicted target genes appeared significantly expressed and negatively correlated to its targeting miRNA (expression profiles are presented in Figure 4).

miRNA target function/cell processes: Target gene functions were grouped according to the cell process to which it relates (Supplementary Table D) and scored as percentage over total cell processes (Table 2). Categories for cell processes were kept similar to the categories used in mRNA analysis as much as possible. As with mRNA analysis, "immune response" and "apoptosis and survival/oxidative stress/DNA damage" were the most dominantly present process categories.

Table 2; Cell processes of target genes of oppositely regulated miRNAs after intake of $4 \mathrm{~g}$ of APAP

Cell processes of significantly regulated and oppositely correlated miRNA target genes as described by GeneCards V3, scored as \% of total target genes

\begin{tabular}{|l|l|}
\cline { 2 - 2 } \multicolumn{1}{l|}{} & APAP dose/time point \\
\hline Cell process & $\mathbf{4 g} / \mathbf{T 2 5}$ \\
\hline Immune response & 24 \\
\hline Lipid metabolism & 3 \\
\hline Cholesterol transport & 0 \\
\hline Apoptosis and survival/oxidative stress/DNA damage & 12 \\
\hline Drug metabolism/pain & 2 \\
\hline mRNA/tRNA related & 10 \\
\hline Other processes & 35 \\
\hline Unknown & 15 \\
\hline
\end{tabular}




\section{Discussion}

The currents test for liver toxicity cannot measure APAP exposure without the occurrence of liver toxicity. 'Omics techniques seem promising with respect to their sensitivity and specificity in measuring molecular effects. We therefore hypothesize that 'omics techniques are sensitive and specific enough to measure the effects of low, (sub-)therapeutic APAP doses in humans. To test this hypothesis, transcriptomic (mRNA and miRNA) and metabolomic analyses were performed on blood samples (transcriptomics) and serum/urine samples (metabolomics) from healthy volunteers taking a low doses of APAP over 24h. In addition, classic clinical liver toxicity tests where performed. While no effects of APAP intake could be detected by any of the classic liver tests, 'omics techniques were able to identify responses to low APAP doses which were similar to those seen after high APAP doses known to cause adverse health effects.

Metabolomic analysis demonstrated that urine samples contained more APAP-derived metabolites in comparison to serum samples. This can be explained by the fact that metabolites accumulate in urine. NAPQI, hydroxy-APAP and methoxy-APAP are oxidative metabolites that all have been associated with hepatotoxic effects of APAP $[21,42,43]$. Especially NAPQI-protein adducts are believed to be key metabolites in causing inflammation-mediated liver toxicity through oxidative stress after exposure to high doses of APAP [42]. NAPQIprotein adducts can be detected in blood/urine when they are released from hepatic cells after lyses due to severe toxicity. Although no NAPQI, methoxy-APAP or hydroxy-APAP metabolites itself were identified within this study, most likely because of fast conversion due to their reactive nature, their derived structures were readily detectable. With an increase in APAP-dose, relatively more of the cysteine-, hydroxy- and methoxyderived metabolites were detected (especially in urine). In addition, 5 so far unknown APAP metabolites were detected; 3 glucuronide products, an APAP-sulphate conjugate and what seems to be an APAPtryptophan product. The concentration of these metabolites in general seems to increase with APAP dose and time (especially in urine). Further elucidation of the exact structure of these metabolites could possibly add to our understanding of the still partially unknown mechanism-ofaction of acetaminophen. So, far we do not know of any other study describing as many metabolites at such low APAP dose levels.

Whole-blood mRNA expression demonstrates dose-related molecular effects after intake of APAP doses $\geq 2 \mathrm{~g} / 24 \mathrm{~h}$. The early 
response (T1/T7) to an APAP dose of $2 \mathrm{~g} / 24 \mathrm{~h}$ mainly involves the regulation of processes related to "drug metabolism/pain" and "immune response" as well as some "lipid metabolism" processes (see Table 1). Closer evaluation of the actual pathways involved in these cell processes revealed that many significantly altered pathways can be directly linked to the known efficacy of APAP. Among others, these pathways involve prostaglandin-E2 and prostaglandin-1/-2 biosynthesis. APAP is believed to exert its effect through cyclooxygenase-1/-2 and possibly cyclooxygenase-3 $[44,45]$. Arachidonic acid is released in the early stages after trauma of cellular membranes. Cyclooxygenase enzymes transform arachidonic acid to prostaglandins, which in turn cause pain and fever.

At T25 after intake of $2 \mathrm{~g}$ of APAP, the affected cell processes shifted towards processes related to an immune response. Among the immune-related processes are macrophage inhibitory factor (MIF)mediated glucocorticoid regulation, signaling of interleukin (IL)-1, IL-17, IL-23 and CD40, cytokine production by T-helper (Th)17 cells and Th17 differentiation. MIF has been suggested to act as a protoxicant signal in drug-induced liver injury [46]. Th17 cells have been described only recently and were shown to be potent inducers of tissue inflammation. Also Th17 cells have been linked to several liver diseases including alcohol-induced liver disease, non-alcoholic steatohepatitis and hepatitis [47]. Th17 cells are known to secrete IL-17, a very potent pro-inflammatory cytokine [48]. However, also other immune cells like macrophages and neutrophils are know to produce IL-17 [49, 50]. One study has mentioned an increased serum concentration of IL-17 in patients with APAP-induced acute liver failure, but IL-17 concentrations showed high inter-individual variability and were not uniformly present [51]. Besides IL-17, also IL-1, IL-23 and CD40 are among the regulated processes after $2 \mathrm{~g}$ APAP at T25, which can all be linked to Th17 cells since their expression seems to be required for the differentiation of Th17 cells $[48,52]$. In addition, these interleukins also have other important roles in the regulation of the immune system and are therefore not exclusively linked to Th17 cells. For instance, IL-1 specifically has been proposed to be a biomarker for liver toxicity [53, 54]. Although several immune-modulating effects of APAP in humans have been suggested before [55-59], no definitive conclusion about possible immunotoxic effects of APAP can be drawn from the present study. Nevertheless, there are clear indications that responses from the immune system, similar to those that would be expected after high, toxic doses of APAP, are triggered after intake of a low, therapeutic dose of APAP. 
At T25 after $4 \mathrm{~g}$ of APAP, a shift appears towards a different response. This response includes processes involved in "apoptosis and survival/oxidative stress/DNA damage". "In fact, the most significantly regulated process at the latter dose is "oxidative phosphorylation", which is down-regulated. In general, liver toxicity due to high, toxic APAP doses is attributed to oxidative stress and mitochondrial dysfunction caused by reactive metabolites formed during drug metabolism. This process is associated with the down-regulation of genes involved in oxidative phosphorylation $[22,60,61]$, as is also seen within this study. Therefore, our study indicates that therapeutic APAP doses cause gene-expression changes in oxidative stress-related processes which may indicate a toxicological risk for liver injury. A recent study focusing on the effects of a bolus dose of $4 \mathrm{~g}$ of APAP on gene expression changes in human blood cells, confirms our findings in that APAP modifies the transcriptome signature by down-regulation of oxidative phosphorylation genes [22]. The authors from this study concluded that these changes resembled those observed in patients who overdosed on APAP and also those changes seen in rats receiving toxic APAP doses. These findings support the idea of a possible pretoxic response to low, therapeutic doses of APAP proceeding, but eventually not resulting in actual profound liver injury. The liver is a robust, regenerative organ with a large over-capacity in a normal, healthy human. Therefore low levels of liver toxicity, if occurring, would probably be dealt with by this organ without any notable complications for the individual.

MiRNAs target mRNAs, which usually induces translational repression and mRNA instability. Based on target gene mRNA product analyses, processes affected by miRNAs at T25 after $4 \mathrm{~g}$ of APAP showed a very similar pattern as those found after mRNA pathway analyses. Regulated miRNAs appeared to be associated with "apoptosis and survival / oxidative stress / DNA damage" and "immune response". Thereby, the miRNA data confirmed the findings on mRNA level.

So far we know of only 2 rodent studies and 1 human study exploring the effects of toxic APAP doses on miRNA expression levels [25-27]. MiRNAs are suggest to have an important role in toxicological mechanisms and consequently are proposed to represent key molecules for toxicity expression [25]. Also, circulating miRNAs are suggested to be sensitive and informative biomarkers for drug-induced liver injury $[26,27]$. In both before mentioned rodent studies, changes on miRNA level were visible before any other toxicological changes in morphology, ALT or lactate could be detected. After a high APAP dose, miR-29c and 
miR-19b were both down-regulated in mouse liver, while in the plasma both these miRNAs were up-regulated [26]. Our study demonstrated both miR-19b and miR-29c to be up-regulated in blood cells after a relatively low APAP dose. Furthermore, miR-542-3p was shown to be up-regulated in rat livers [25], which is comparable to our results obtained in blood after $4 \mathrm{~g}$ of APAP. In humans, hsa-miR-122 and hsamiR-192 circulating in blood plasma have been suggested as possible biomarkers for acute liver injury induced by high APAP dose ingestion [27]. Both miRNAs were significantly up-regulated in plasma of patients suffering from APAP induced acute liver injury, i.e. in patients with elevated ALT levels. Most likely, these miRNAs are released from the liver due to toxic insults. Especially hsa-miR-122, which expression is liver specific, would otherwise not occur in plasma [62]. In the current study, ALT levels were not elevated and neither were hsa-miR-122 nor hsa-miR-192 levels. Although hsa-miR-192 did almost pass the selection criteria for significance with a fold change of 1.76 and a p-value of 0.097. In the current study the expression pattern of miRNAs in blood cells was included, which is not the case in the study of Starkey Lewis et al. Therefore the outcome of both studies cannot be directly compared. Nevertheless, the increase of hsa-miR-192 in plasma could (partly) be caused by increased expression of this miRNA in white blood cells and leakage from these cells due to toxicity. This phenomenon seems to precede leakage of hsa-miR-122 from liver cells. Taken together, not only on the metabolomic and mRNA level, but also on the miRNA level, responses to low, therapeutic APAP doses resembled those seen after high, toxic APAP doses.

In summary, we have shown that 'omics techniques are able to measure a response to short term, low dose APAP intake by human volunteers on mRNA and miRNA in blood cells and on serum and urine metabolite level. 'Omics thereby outperforms the classical clinical chemistry tests which were not able to detect any response to low dose APAP exposure. Furthermore, several new APAP metabolites which have not yet been described in literature were identified. Intake of $2 \mathrm{~g}$ of APAP resulted in a diverse metabolite pattern, including cysteine-, hydroxyand methoxy-metabolites that have been associated with APAP-related hepatotoxicity after high APAP dose exposure. Transcriptomic changes indicated dose-specific immune-modulating effects, which cannot be fully explained yet, but at least show that low dose APAP exposure does trigger an immune response. This response could possibly be related to a mild version of liver damage, but eventually does not lead to severe liver injury. A possibly cause for liver damage after low APAP doses could be related to the oxidative APAP metabolites associated to 
hepatotoxicity that were measured at these doses. $24 \mathrm{H}$ after ingestion of $4 \mathrm{~g}$ of APAP, a metabolomic response very similar as seen after $2 \mathrm{~g}$ was detected, but with higher levels of hepatotoxicity-related metabolites. An increase in oxidative metabolite levels is associated with the generesponse specific for this dose demonstrating changes in oxidative stress-related pathways. In addition, effects of APAP administration on miRNA expression confirmed the findings on mRNA level concerning both the effects of APAP on immune responses and the effects of APAP on apoptosis/oxidative stress/DNA damage.

The individual factors which influence APAP susceptibility remain still partially unknown. Our study - having in itself insufficient numbers of volunteers to evaluate this - may provide a model for exploring such inter-individual variability in drug responses, since the data revealed a wealth of biological information indicating effects both related to efficacy and possible toxicity of APAP. Therefore, studies like this one could help in finding answers to the questions that are currently posted about the social and medical issues around APAP use and its still partially unknown mechanism-of-action. However, larger sample numbers will then have to become available.

\section{Supplementary Tables}

Supplementary Tables A-D: Available online: http://www. sciencedirect.com/science/article/pii/S0041008X12000208

\section{Acknowledgments}

This work was supported by the Dutch Ministry of Public Health, Welfare and Sports (VWS) as a part of the Assuring Safety without Animal Testing (ASAT) initiative. Furthermore, we would like to thank Dr. K.W.H. Wodzig of the clinical chemistry laboratory from the University Hospital Maastricht for performing the clinical liver chemistry analyses. 


\section{References}

1. Lee, W.M., Acetaminophen toxicity: changing perceptions on a social/medical issue. Hepatology, 2007. 46(4): p. 966-70.

2. Kuehn, B.M., FDA focuses on drugs and liver damage: labeling and other changes for acetaminophen. JAMA, 2009. 302(4): p. 369-71.

3. FDA Summary Minutes of the Joint Meeting of the Drug Safety and Risk Management Advisory Committee, Nonprescription Drugs Advisory Committee, and the Anesthetic and Life Support Drugs Advisory Committee June 29 and 30. 2009.

4. FDA FDA Requires Additional Labeling for Over-the-Counter Pain Relievers and Fever Reducers to Help Consumers Use Products Safely. 2009.

5. FDA Prescription Drug Products Containing Acetaminophen: Actions to Reduce Liver Injury from Unintentional Overdose. 2011.

6. Watkins, P.B., et al., Aminotransferase elevations in healthy adults receiving 4 grams of acetaminophen daily: a randomized controlled trial. JAMA, 2006. 296(1): p. 87-93.

7. Watkins, P.B., Biomarkers for the diagnosis and management of drug-induced liver injury. Semin Liver Dis, 2009. 29(4): p. 393-9.

8. Harrill, A.H. and I. Rusyn, Systems biology and functional genomics approaches for the identification of cellular responses to drug toxicity. Expert Opin Drug Metab Toxicol, 2008. 4(11): p. 1379-89.

9. Vlaanderen, J., et al., Application of OMICS technologies in occupational and environmental health research; current status and projections. Occup Environ Med, 2010. 67(2): p. 136-43.

10. Beger, R.D., J. Sun, and L.K. Schnackenberg, Metabolomics approaches for discovering biomarkers of drug-induced hepatotoxicity and nephrotoxicity. Toxicol Appl Pharmacol, 2010. 243(2): p. 154-66.

11. Vinayavekhin, N., E.A. Homan, and A. Saghatelian, Exploring disease through metabolomics. ACS Chem Biol, 2010. 5(1): p. 91-103.

12. Bushel, P.R., et al., Blood gene expression signatures predict exposure levels. Proc Natl Acad Sci U S A, 2007. 104(46): p. 18211-6.

13. Kroeger, M., How omics technologies can contribute to the '3R' principles by introducing new strategies in animal testing. Trends Biotechnol, 2006. 24(8): p. 343-6.

14. Coen, M., et al., Integrated application of transcriptomics and metabonomics yields new insight into the toxicity due to paracetamol in the mouse. J Pharm Biomed Anal, 2004. 35(1): p. 93-105.

15. Beyer, R.P., et al., Multicenter study of acetaminophen hepatotoxicity reveals the importance of biological endpoints in genomic analyses. Toxicol Sci, 2007. 99(1): p. 326-37.

16. Heinloth, A.N., et al., Gene expression analysis offers unique advantages to histopathology in liver biopsy evaluations. Toxicol Pathol, 2007. 35(2): p. 276-83.

17. Kikkawa, R., et al., In vivo hepatotoxicity study of rats in comparison with in vitro hepatotoxicity screening system. J Toxicol Sci, 2006. 31(1): p. 23-34.

18. Aleksunes, L.M., et al., Influence of acetaminophen vehicle on regulation of transporter gene expression during hepatotoxicity. J Toxicol Environ Health A, 2007. 70(21): p. 1870-2.

19. Morishita, K., et al., Gene expression profile in liver of differing ages of rats after single oral administration of acetaminophen. J Toxicol Sci, 2006. 31(5): p. 491-507. 
20. Chen, C., et al., Serum metabolomics reveals irreversible inhibition of fatty acid beta-oxidation through the suppression of PPARalpha activation as a contributing mechanism of acetaminophen-induced hepatotoxicity. Chem Res Toxicol, 2009. 22(4): p. 699-707.

21. Chen, C., et al., Identification of novel toxicity-associated metabolites by metabolomics and mass isotopomer analysis of acetaminophen metabolism in wild-type and Cyp2e1-null mice. J Biol Chem, 2008. 283(8): p. 4543-59.

22. Fannin, R.D., et al., Acetaminophen dosing of humans results in blood transcriptome and metabolome changes consistent with impaired oxidative phosphorylation. Hepatology, 2010. 51(1): p. 227-36.

23. Umbright, C., et al., Blood gene expression markers to detect and distinguish target organ toxicity. Mol Cell Biochem, 2010. 335(1-2): p. 223-34.

24. Huang, J., et al., Genomic indicators in the blood predict drug-induced liver injury. Pharmacogenomics J, 2010. 10(4): p. 267-77.

25. Fukushima, T., et al., Changes of micro-RNA expression in rat liver treated by acetaminophen or carbon tetrachloride--regulating role of micro-RNA for RNA expression. J Toxicol Sci, 2007. 32(4): p. 401-9.

26. Wang, K., et al., Circulating microRNAs, potential biomarkers for drug-induced liver injury. Proc Natl Acad Sci U S A, 2009. 106(11): p. 4402-7.

27. Starkey Lewis, P.J., et al., Circulating microRNAs as potential markers of human drug-induced liver injury. Hepatology, 2011. 54(5): p. 1767-1776.

28. Lommen, A., et al., Ultra-fast searching assists in evaluating sub-ppm mass accuracy enhancement in U-HPLC/Orbitrap MS data. Metabolomics, 2011. 7(1): p. 15-24.

29. Lommen, A., et al., On the detection of environmental effects on complex matrices combining off-line liquid chromatography and $1 \mathrm{H}-\mathrm{NMR}$.

Biodegradation, 1998. 9(6): p. 513-525.

30. Lommen, A., MetAlign: interface-driven, versatile metabolomics tool for hyphenated full-scan mass spectrometry data preprocessing. Anal Chem, 2009. 81(8): p. 3079-86.

31. Bales, J.R., et al., Urinary excretion of acetaminophen and its metabolites as studied by proton NMR spectroscopy. Clin Chem, 1984. 30(10): p. 1631-6.

32. Rijk, J.C., et al., Metabolomics approach to anabolic steroid urine profiling of bovines treated with prohormones. Anal Chem, 2009. 81(16): p. 6879-88.

33. Ruiz Aracama, A., et al., An untargeted multi-technique metabolomics approach to study intracellular metabolites of HepG2 cells exposed to 2,3,7,8tetrachlorodibenzo-p-dioxin. Submitted, 2011.

34. van lersel, M.P., et al., Presenting and exploring biological pathways with PathVisio. BMC Bioinformatics, 2008. 9: p. 399.

35. Daykin, C.A., et al., The comparison of plasma deproteinization methods for the detection of low-molecular-weight metabolites by (1)H nuclear magnetic resonance spectroscopy. Anal Biochem, 2002. 304(2): p. 220-30.

36. Livak, K.J. and T.D. Schmittgen, Analysis of relative gene expression data using real-time quantitative PCR and the 2(-Delta Delta C(T)) Method. Methods, 2001. 25(4): p. 402-8.

37. Bioinformatics Wageningen UR, European Nutrigenomics Organisation (NuGO). MADMAX. Available from: https://madmax.bioinformatics.nl/pls/apex/ $f ? p=100: 1$.

38. University of Michigan. Brainarray custom CDF. version 13; Available from: http://brainarray.mbni.med.umich.edu/Brainarray/Database/CustomCDF/ cdfreadme.htm.

39. Shi, L., et al., The MicroArray Quality Control (MAQC) project shows inter- and 
intraplatform reproducibility of gene expression measurements. Nat Biotechnol, 2006. 24(9): p. 1151-61.

40. Tusher, V.G., R. Tibshirani, and G. Chu, Significance analysis of microarrays applied to the ionizing radiation response. Proc Natl Acad Sci U S A, 2001. 98(9): p. 5116-21.

41. Bates, D., et al., The R Project for Statistical Computing.

42. Dahlin, D.C., et al., N-acetyl-p-benzoquinone imine: a cytochrome P-450mediated oxidation product of acetaminophen. Proc Natl Acad Sci U S A, 1984. 81(5): p. 1327-31.

43. Wilson, J.M., et al., Analysis of acetaminophen metabolites in urine by high-performance liquid chromatography with UV and amperometric detection. J Chromatogr, 1982. 227(2): p. 453-62.

44. Vane, J.R., Inhibition of prostaglandin synthesis as a mechanism of action for aspirin-like drugs. Nat New Biol, 1971. 231(25): p. 232-5.

45. Davies, N.M., et al., Cyclooxygenase-3: axiom, dogma, anomaly, enigma or splice error?--Not as easy as 1, 2, 3. J Pharm Pharm Sci, 2004. 7(2): p. 217-26.

46. Bourdi, M., et al., Protection against acetaminophen-induced liver injury and lethality by interleukin 10: role of inducible nitric oxide synthase. Hepatology, 2002. 35(2): p. 289-98.

47. Hammerich, L., F. Heymann, and F. Tacke, Role of IL-17 and Th17 cells in liver diseases. Clin Dev Immunol, 2011. 2011: p. 345803.

48. Korn, T., et al., IL-17 and Th17 Cells. Annu Rev Immunol, 2009. 27: p. 485-517.

49. Song, C., et al., IL-17-producing alveolar macrophages mediate allergic lung inflammation related to asthma. J Immunol, 2008. 181(9): p. 6117-24.

50. Benghiat, F.S., et al., Interleukin 17-producing $T$ helper cells in alloimmunity. Transplant Rev (Orlando), 2009. 23(1): p. 11-8.

51. Li, J., et al., Cytokine and autoantibody patterns in acute liver failure. J Immunotoxicol, 2010. 7(3): p. 157-64.

52. lezzi, G., et al., CD40-CD40L cross-talk integrates strong antigenic signals and microbial stimuli to induce development of IL-17-producing CD4+ T cells. Proc Natl Acad Sci U S A, 2009. 106(3): p. 876-81.

53. Lacour, S., et al., Cytokines as potential biomarkers of liver toxicity. Cancer Biomark, 2005. 1(1): p. 29-39.

54. Akbay, A., et al., Serum cytotoxin and oxidant stress markers in $\mathrm{N}$-acetylcysteine treated thioacetamide hepatotoxicity of rats. Hum Exp Toxicol, 1999. 18(11): p. 669-76.

55. Doran, T.F., et al., Acetaminophen: more harm than good for chickenpox? J Pediatr, 1989. 114(6): p. 1045-8.

56. Graham, N.M., et al., Adverse effects of aspirin, acetaminophen, and ibuprofen on immune function, viral shedding, and clinical status in rhinovirus-infected volunteers. J Infect Dis, 1990. 162(6): p. 1277-82.

57. Baken, K.A., et al., Overlapping gene expression profiles of model compounds provide opportunities for immunotoxicity screening. Toxicol Appl Pharmacol, 2008. 226(1): p. 46-59.

58. Harrill, A.H., et al., Mouse population-guided resequencing reveals that variants in CD44 contribute to acetaminophen-induced liver injury in humans. Genome Res, 2009. 19(9): p. 1507-15.

59. Prymula, R., et al., Effect of prophylactic paracetamol administration at time of vaccination on febrile reactions and antibody responses in children: two open-label, randomised controlled trials. Lancet, 2009. 374(9698): p. 1339-50.

60. Han, D., et al., Signal transduction pathways involved in drug-induced liver 
injury. Handb Exp Pharmacol, 2010(196): p. 267-310.

61. Katyare, S.S. and J.G. Satav, Impaired mitochondrial oxidative energy metabolism following paracetamol-induced hepatotoxicity in the rat. $\mathrm{Br}$ J Pharmacol, 1989. 96(1): p. 51-8.

62. Liang, Y., et al., Characterization of microRNA expression profiles in normal human tissues. BMC Genomics, 2007. 8: p. 166. 


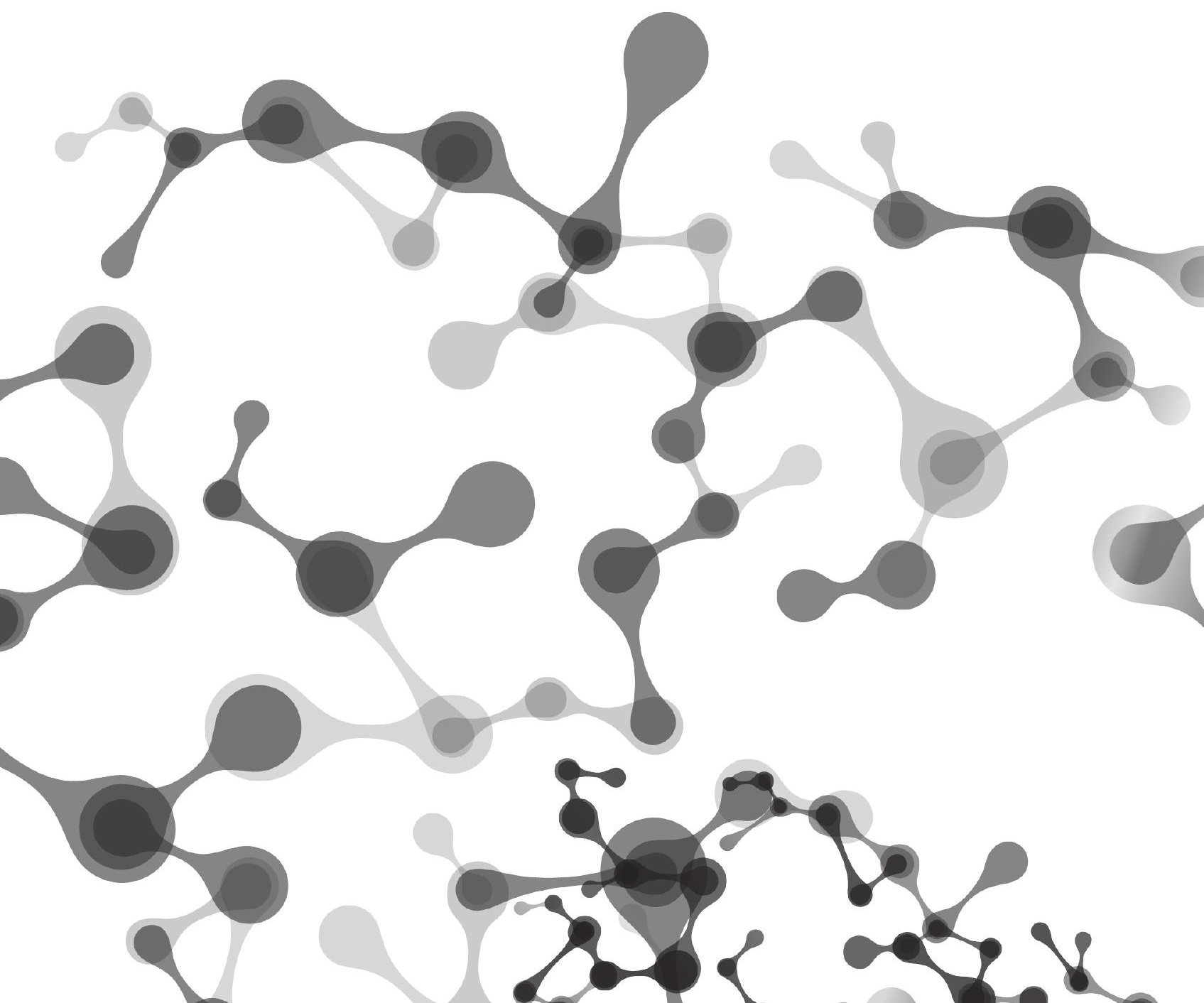




\section{Chapter 7}

Summary and general discussion Samenvatting en algemene discussie 
Interindividual variation in response to the exposure to chemical compounds is a well-known phenomenon within the human population. [1-3]. Amongst others, genetic factors have been proposed as causative factors for human interindividual variation [1, 2]. For instance, interindividual variation in cytochrome P450 (CYP) enzyme expression and/or activity has been linked to idiosyncratic responses to drugs and to susceptibility to carcinogenesis [4-7]. Nevertheless, the variation in the before mentioned factors seems not enough to fully explain the variation in responses between individuals. Therefore, we assume that many of the causative factors for interindividual variation and the molecular mechanisms underlying this variation are still unknown.

In addition, interindividual variation in responses to compound exposure is a major complicating factor in human risk-estimation procedures [8]. In an attempt to include interindividual variation in the process of risk-estimation for a compound (excluding genotoxic carcinogens), the 100-fold safety factor has been postulated [9]. This factor is composed of two separate factors both representing a 10-fold safety margin. The first 10 -fold margin is applied to extrapolate animalderived data, the current golden standard in toxicological research, to the human situation, assuming that humans are more susceptible to toxicity than animals $[10,11]$. Then, another 10 -fold margin is applied that should compensate for the interindividual variation in susceptibility to induced toxicity within the human populations [9, 12]. As such, this 100-fold factor should allow the animal-based estimation of a dose for a particular compound that can be considered safe for humans when exposed on daily basis. However, the 10-fold factor for interindividual variation in susceptibility within the human population is currently under discussion [13]. Better knowledge of the variation within the human population as such and in the molecular mechanisms underlying adverse responses to compounds may help to develop less arbitrary procedures for human risk estimate.

In addition, the use of animals for toxicity testing comes with ethical and moral dilemmas in addition to a heavy financial burden [14]. Also, the reliability and applicability of animal model-derived data towards the human situation has been questioned over the recent past [14]. Furthermore, rodent laboratory models are inherent to an inbred genetic background and thus by default, do not allow for the evaluation of interindividual variation within the human population. Overall, the above mentioned issues demonstrate the need for, better, in vitro models preferably optimised for application to the human situation. 
The liver is the major organ involved in the bio-activation of chemical compounds into reactive metabolites. Therefore, this organ is the main candidate for the development of human in vitro models for the assessment of compound exposure-related toxicity responses [15]. Several liver carcinoma-derived cell lines are available and these are regularly used in toxicological research [16-20]. However, these cell lines do not allow for the evaluation of interindividual variation. Since the hepatocyte is the main cell type in the liver, these cells are of special interest for the development of an in vitro model that aims at representing the human liver [21-23]. Also, since primary human hepatocytes $(\mathrm{PHH})$ can be isolated from any viable human liver donor tissue, evaluation of interindividual variation in toxic responses is feasible. Yet another in vitro model for the human liver is represented by precision-cut human liver tissue slices. In this model, the natural integrity of the liver is not disturbed and all liver cell types (so, not only hepatocytes) are represented [21]. As is the case with primary human hepatocytes, slices can be prepared from any available, viable human liver tissue and enables the possibility to determine interindividual variation.

In addition to better cellular models for evaluating toxic reactions, also better techniques to study genome-wide responses to compound exposure have become available; the so-called 'omics or array-based techniques. These techniques promise a higher sensitivity than conventional toxicological assays. They should be able to detect responses to compound exposure at lower doses even before the onset of a phenotypic response, thus increasing the predictive value of such assays [24-27]. Also, due to their ability to measure genomewide responses, these technologies allow us to study the molecular mechanisms underlying compound-related responses. Chips to study gene expression were among the first type of arrays that became available, but these have now been complemented with, amongst others, miRNA and gene methylation platforms thus enabling the study of epigenetic effects of compound exposure complimentarily to responses at the gene expression level.

In this thesis, our hypothesis has been that 'omics data derived from in vitro models for the human liver will enable us to pinpoint genes/gene sets that predispose for increased susceptibility of an individual upon being challenged by xenobiotics. Also, implementing epigenomics techniques should enable us to evaluate whether the epigenetic status of an individual influences the susceptibility of this individual to toxic challenges. 


\section{Evaluation of interindividual variation using precision cut human liver slices}

In Chapter 2 we have used precision cut human liver slices to evaluate whether interindividual variation in baseline enzyme activity (EA) and gene expression (GE) levels in the liver predispose for the variation in toxicity responses, based on assessing dose-response relationships for several prototypical hepatotoxicants. Baseline levels of CYP GE and EA were measured in precision-cut human liver slices. In addition, slices were exposed to a dose-range of acetaminophen (APAP), aflatoxin B1 (AFB), benzo( $\alpha$ )pyrene (BaP) or 2-nitrofluorene (NF). Interindividual variation in induced genotoxicity (COMET-assay and CDKN1A/p21 GE) and cytotoxicity (lactate dehydrogenase-leakage), combined with NQO1- and GSTM1-induced GE-responses for oxidative stress and GE-responses of several CYPs, was evaluated. The benchmark dose (BMD)-approach was applied as a tool to model exposure responses on an individual level.

Variation in baseline CYP levels, both GE and EA, appears to explain the variation in compound exposure-responses on an individual level. Network analyses enable the definition of key parameters influencing interindividual variation after compound exposure. For NF, this analysis suggests involvement of CYP1B1 in the metabolism of this compound, which represents a novel finding. Furthermore, GSTM1 which is known to be highly polymorphic in expression within the human population, but so far could not be linked to toxicity in APAPpoisoned patients, is suggested to be associated with interindividual variability in APAP-metabolism, depending on the individual's expression levels of CYP-enzymes.

This study demonstrates that using interindividual variation within network modelling provides a method for the definition of essential and even new parameters involved in compound-related metabolism. Out of all compounds, the interindividual variation in the cytotoxicity response derived BMDs was the largest after APAP-exposure (400-fold). This high interindividual variability corresponds with reports from literature on high variability within the human population with respect to sensitivity for toxicity responses to APAP [28]. In addition, this variation well exceeds the 10-fold margin to correct for interindividual variation that is currently taken into account in risk-estimate procedures. This information stresses the need for a more thorough evaluation of interindividual variation in toxic response, in the context of the necessity to improve the quantitative estimate of human risks. 
For $\mathrm{AFB}, \mathrm{BaP}$ and $\mathrm{NF}$ the fold-differences in interindividual variation in the cytotoxicity response derived BMDs were much lower $(6,54$, and 4-fold respectively).

\section{Interindividual variation in primary human hepatocytes}

Chapter $\mathbf{3}$ focusses on the interindividual variation in gene expression responses and metabolite formation in primary human hepatocytes (PHH) exposed to APAP. APAP is a readily available overthe-counter drug and is one of the most commonly used analgesics/ antipyretics world-wide [29, 30]. Although large interindividual variation in susceptibility towards APAP-induced liver failure has been described, the exact underlying factors causing this variability in susceptibility are still largely unknown [31, 32].

To better understand this variability in response to APAP, interindividual differences in gene expression changes and APAPmetabolite formation in $\mathrm{PHH}$ from several donors exposed in vitro to a non-toxic to toxic APAP dose-range were evaluated. The interindividual variation in gene expression levels was correlated with the interindividual variation in APAP-metabolite levels to evaluate whether differences in gene expression levels could explain the differences in levels of APAPmetabolite formation. Gene set over-representation analysis was applied to enable biological interpretation of the transcriptomic results.

The biological processes in which the genes with high interindividual variation in expression are involved, include liver regeneration, inflammatory responses, mitochondrial stress responses, hepatocarcinogenesis, cell cycle and drug-efficacy. Additionally, the interindividual variation in the expression of these genes could be associated with the variability in the metabolite levels of hydroxylAPAP, methoxy-APAP and C8H1305N-APAP-glucuronide. These genes/ metabolites may thus be of interest with respect to drug-induced liver injury, especially when they can be confirmed in a human in vivo situation as is described in Chapter 6. In addition, interindividual variation in gene expression levels in general appeared to exceed the variation in metabolite levels.

As mentioned before, cell lines are currently widely used as an in vitro model for the human liver in toxicological research. However, these cell lines do not allow for the evaluation of interindividual variation. In

Chapter 4 we evaluate to what extent the data derived from several cell lines, is similar to the data derived from $\mathrm{PHH}$, an in vitro model that 
does include interindividual variation. While previous studies have mainly focused on the comparability/applicability of these cell types based on their baseline biotransformation capacities/CYP-inducibility [33-35], we chose for a genome-wide gene expression profile- based approach both at baseline and at compound-induced gene expression levels. Therefore, we compared baseline and AFB- and BaP-induced gene expression profiles in HepG2, HepaRG and PHH.

At baseline, liver models differ from each other with respect to whole genome gene expression levels. $\mathrm{PHH}$ are characterized, as expected, by profound interindividual differences, and are most similar to HepaRG. After compound exposure, induced gene expression profiles are more similar between cell models, especially upon BaP treatment. Pathways involved in compound metabolism, are induced in all 3 models, while others are more pronounced in a specific cell model. Examples are transcriptomic modifications of carbohydrate-related genes (HepaRG) and of receptor-related genes $(\mathrm{PHH})$ after BaP exposure, and of cell cycle-related genes (HepG2) after AFB treatment. Thus, at baseline level, PHH are more similar to HepaRG than to HepG2, but for toxicogenomics applications both cell lines perform equally well in comparison to $\mathrm{PHH}$. However, the interindividual variation in the $\mathrm{PHH}$ model provides a much broader and more heterogeneous picture of the responses to compound exposure. Since this variation cannot be captured by any of the cell line models, their application in toxicity-risk evaluation implies an incomplete evaluation in relation to the human population.

Now that we have established clear interindividual differences in baseline transcriptomics and in compound-induced gene expression levels, the question remains what causes these interindividual responses. The influence of epigenetic factors, e.g. gene-methylation, has been proposed as an additional factor that could explain the differences in chemically induced gene expression levels in the human population. Therefore, in Chapter $\mathbf{5}$ we focus on determining to what extent the baseline methylation status of a gene influences the expression of that gene in response to compound exposure. In this context, special emphasis has been put on the interindividual variation of the DNAdamage response.

In primary human hepatocytes of several donors exposed to AFB or BaP (see Chapter 4) the response to DNA-damage was evaluated by $\mu \mathrm{H} 2 \mathrm{Ax}$-staining. Results were used to split the study-population into two groups per compound; a low and a high responder group, based 
on the BMD for each response. Genes unique in their gene expression responses for either one of these groups were evaluated with regard to their baseline methylation status and baseline gene expression levels.

Although clear differences in responses to DNA-damage between high and low responders could be defined, baseline methylation status seems to only marginally contribute to this variation. Also, interindividual variation in baseline gene expression levels does not seem to be related to the variation between individuals in baseline methylation levels, which in turn is not reflected in the interindividual variation in gene expression responses. Thus, the general conclusion is that the interindividual variation in baseline methylation status only provides limited information on the susceptibility to carcinogen exposure of groups/individuals. Possibly, the regulatory mechanism through gene methylation is more complicated than currently acknowledged. Including other (epi)genetic factors, such as the influence of miRNAs, may help our interpretation and understanding of interindividual variation.

\section{Interindividual variation in human in vivo responses}

Although in vitro human liver models so far have been proven useful to evaluate interindividual variation, the ultimate model is of course the human liver in vivo. However due to obvious ethical constraints, intentional exposures to toxic doses of a compound cannot be performed using this model. Furthermore, it is evident that due to its anatomical position, accessibility to the liver is limited. However, blood has been proven as a useful reference or surrogate tissue to monitor liver function, including the response to xenobiotic exposure [36-38].

In Chapter 6 we have evaluated the interindividual differences in blood cell transcriptomics responses of several volunteers exposed to relatively low, therapeutic doses of APAP. A battery of standard clinical liver chemistry tests was used to monitor for possible signs of liver damage. A procedure similar to the one used in Chapter $\mathbf{2}$ was applied to evaluated gene expression changes after APAP exposure. Also, interindividual variation in APAP-metabolite formation in blood and urine was determined. To our knowledge, this is the first study to evaluate full-genome human miRNA expression changes in blood cells from healthy human volunteers exposed to APAP.

Many known and several new APAP-metabolites could be detected, in particular in relation to the oxidative route of APAP 
metabolism which is often linked to the hepatotoxicity-related effects of APAP. Also, the metabolites mentioned in Chapter $\mathbf{2}$ as being of possible interest in the perspective of drug-induced liver injury, are confirmed in this in vivo study. Transcriptomic changes indicate immunemodulating effects and oxidative stress responses to APAP.

'Omics techniques outperform clinical chemistry tests and reveal novel response pathways to APAP in humans. Although no definitive conclusion about potential immuno-toxic effects of APAP can be drawn from this study, there are clear indications that the immune system is triggered even after intake of low doses of APAP. Also, oxidative stressrelated gene expression responses, similar to those seen after high dose APAP exposure, suggest the occurrence of possible pre-toxic effects of therapeutic APAP-doses. Possibly, these effects are related to dosedependent increases in levels of hepatotoxicity-related metabolites. In addition, the interindividual variation in miRNA expression patterns can be related to the interindividual variation in gene expression responses. Therefore, miRNAs should be considered as a useful addition to the knowledge needed to explain the interindividual variation within the human population.

\section{Conclusions}

Network analysis (Chapter 2) proved useful in defining baseline CYP gene expression and enzyme activity levels as key parameters influencing interindividual variation after compound exposure. The high interindividual variation in metabolizing genes, mainly CYPs, could be confirmed in Chapter 5.

At baseline gene expression levels, $\mathrm{PHH}$ are very different from HepaRG and HepG2, two carcinoma-derived cell models that are currently used in toxicity testing (Chapter 4). PHH, HepaRG and HepG2 become more similar with respect to their gene expression profiles after compound exposure. Nevertheless, the $\mathrm{PHH}$-model gives a much broader and more heterogeneous picture of the responses to compound exposure due to the inclusion of the interindividual variation between donors.

Interindividual variation in gene expression responses could be detected in all non-carcinoma based in vitro and in vivo liver models applied in this thesis (Chapter 2, $\mathbf{3}$ and 6). Interindividual variation in some parameters is found to exceed the 10-fold margin that is supposed to represent a measure for interindividual variation within the 
human population in toxicity testing. These factors include BMDs for gene expression responses and cytotoxicity responses, but also levels of metabolite formation. In addition, inclusion of data on interindividual variation in miRNA expression levels could prove helpful in an attempt to explain the large variation between individuals in responses to APAP exposure in the human population (Chapter 6).

The combination of multiple 'omics techniques (e.g. genomics and proteomics) has been proven very useful in defining metabolites that could be used to monitor interindividual variation in the response to APAP-exposure (Chapter $\mathbf{3}$ and 6). The addition of epigenetic data in an attempt to explain interindividual variation was more successful with the addition of information on miRNA expression than with the addition of methylation-related data (Chapter $\mathbf{5}$ and 6).

Overall, the research performed in this thesis confirms the occurrence of considerable interindividual variation in responses to toxic exposures within the human population. Baseline CYP enzyme activity and gene expression levels as well as compound-induced genotoxicity, cytotoxicity and gene expression levels all show clear variation between liver cells from individual donors. In our opinion, implementation of knowledge gained on human interindividual variation should lead to the development of less arbitrary procedures for human risk estimate and a better understanding of the molecular mechanisms underlying responses to compounds

\section{Recommendation for future directions}

An increased awareness for the importance of the evaluation of interindividual variation has been established over the years, together with an increased demand for the replacement of animal models. However to be able to fully understand interindividual variation in toxic responses, massive amounts of data from a large group of individuals will need to be collected, in order to be able to create a dataset that reliably represents heterogeneity within the human population. For this a relevant human cell model that also is easily accessible and applicable on a large scale, is needed. In the case of liver models, this is not (yet) available. The existing cell lines that are numerously available and cheap, do not allow for interindividual variation. $\mathrm{PHH}$ and liver slices are not available in large amounts and come with heavy technical demands.

Recently, several new in vitro models have been developed. One of these models is based on hepatocyte-like cells that can be 
derived from the differentiation of human embryonic stem cells [39]. Another similar model is based on induced human pluripotent stem cells [40]. This model has the advantage that the cells derived from the stem cells, should have a genetic background that is close to normal hepatocytes. Furthermore, culture methods have progressed, allowing for the culturing of cells in 3D or micro-tissue formation. These new culture conditions should mimic the natural surroundings of the cell in the liver and should, amongst others, enhance the metabolic capacities of the cells in comparison to the regular 2D cultures [40]. In addition, so called co-cultures which include both primary parenchymal and nonparenchymal hepatic cells have been developed. Also these cell models represent a more in vivo-like representation of the liver with improved functional capabilities [41]. All of the above mentioned new in vitro models for the liver should allow for the evaluation of interindividual variation and thus provide a promising perspective for future advances.

Nevertheless, the largest issue to overcome will be the necessary adjustments of the rules and regulations. This applies to toxicity testing in general, but it will be especially difficult for those rules and regulations that apply to human risk-estimation. Any new model will have to compete against the test that have become the golden standard over-time, namely the animal models. This, without a doubt, will prove to be a very lengthy process. 
Interindividuele variatie in reactie op de blootstelling aan chemische verbindingen is een bekend verschijnsel in de menselijke populatie. [1-3]. Onder andere genetische factoren zijn voorgedragen als oorzakelijke factoren voor deze menselijke interindividuele variatie [1 , 2]. Zo is bijvoorbeeld de interindividuele variatie in cytochroom P450 (CYP) enzym expressie en/of activiteit geassocieerd met idiosyncratische reacties op geneesmiddelen en de gevoeligheid voor carcinogenese [4-7]. Echter, de variatie in de hierboven genoemde factoren lijkt niet voldoende om de variatie in de respons tussen individuen volledig te kunnen verklaren. Daarom gaan we ervan uit dat veel van de oorzakelijke factoren voor interindividuele variatie en de moleculaire mechanismen die ten grondslag liggen aan deze variatie nog onbekend zijn.

Bovendien is de interindividuele variatie in de respons op blootstelling aan stoffen een belangrijke complicerende factor in de procedures betreffende de humane risicobeoordeling [8]. In een poging deze interindividuele variatie te includeren in het proces aangaande de risicobeoordeling van stoffen (genotoxische carcinogenen daargelaten), heeft men een 100-voudige veiligheidsfactor geïntroduceerd [9]. Deze factor is samengesteld uit twee afzonderlijke elementen die beiden een 10-voudige veiligheidsmarge vertegenwoordigen. Allereerst wordt een 10-voudige marge toegepast op proefdier-model gerelateerde data, de huidige gouden standaard in toxicologisch onderzoek, om zo te extrapoleren naar de humane situatie ervan uitgaande dat mensen gevoeliger zijn voor toxiciteit dan dieren [10, 11]. Vervolgens wordt nog een 10-voudige marge toegepast die moet compenseren voor de interindividuele variatie in gevoeligheid voor toxiciteit binnen de humane populatie $[9,12]$. Als zodanig voorziet deze 100-voudige veiligheidsfactor in de mogelijkheid om een schatting te maken van een op dierenmodellen gebaseerde dosis voor een specifieke verbinding die voor mensen als veilig kan worden beschouwd op basis van dagelijkse blootstelling. Echter, in de afgelopen jaren is er controversie ontstaan rondom de 10-voudige factor die zou moeten corrigeren voor de interindividuele variatie in gevoeligheid binnen de humane populatie [13]. Een toename van kennis over de werkelijke interindividuele variatie binnen de humane populatie als zodanig en een beter inzicht in de moleculaire mechanismen onderliggend aan de respons op blootstelling aan stoffen helpen een minder willekeurige risicobeoordeling voor mensen te bewerkstelligen.

Daarnaast gaat het gebruik van proefdieren voor toxicologisch onderzoek gepaard met ethische en morele dilemma's en een zware 
financiële last [14]. Ook is recentelijk discussie ontstaan betreffende de betrouwbaarheid en toepasbaarheid van data afkomstig van proefdiermodellen op de humane situatie [14]. Daarbij zijn knaagdiermodellen inherent verbonden aan een inteelt-gerelateerde genetische achtergrond, welke niet geschikt is voor de evaluatie van interindividuele variatie binnen de humane populatie. Bovengenoemde punten tonen aan dat er behoefte is aan betere, in vitro testmodellen bij voorkeur geoptimaliseerd voor toepassing binnen de humane situatie.

De lever is het belangrijkste orgaan dat is betrokken bij de bio-activatie van chemische verbindingen in reactieve verbindingen. Daarom is dit orgaan de voornaamste kandidaat voor de ontwikkeling van humane in vitro modellen voor het beoordelen van toxische reacties op blootstelling aan stoffen [15]. Verschillende cellijnen verkregen uit levercarcinomen zijn beschikbaar en worden regelmatig gebruikt in toxicologisch onderzoek [16-20]. Echter, deze cellijnen kunnen niet gebruikt worden voor de evaluatie van interindividuele variatie. Aangezien hepatocyten het meest voorkomende celtype in de lever vertegenwoordigen, vormen deze cellen een belangrijke kandidaat bij de ontwikkeling van een in vitro model dat de humane lever moet simuleren [21-23]. Verder, aangezien primaire humane hepatocyten $(\mathrm{PHH})$ kunnen worden geïsoleerd uit ieder levensvatbaar leverweefsel van humane donoren, behoort de evaluatie van interindividuele variatie in toxische reacties tot de mogelijkheden. Een ander in vitro model voor de menselijke lever wordt vertegenwoordigd door precisie-gesneden humane leverslices (plakjes). In dit model wordt de biologische integriteit van de lever niet verstoord en zijn alle verschillende soorten levercellen vertegenwoordigd (dus niet alleen de hepatocyten) [21]. Net als de primaire humane hepatocyten, kunnen deze slices worden bereid van elk beschikbaar, levensvatbaar humaan leverweefsel waardoor de evaluatie van interindividuele variatie mogelijk is.

Naast betere cel modellen voor de evaluatie van toxische reacties zijn ook betere technieken beschikbaar gekomen die een genoombrede aanpak bij het bestuderen van deze reacties op blootstelling aan stoffen mogelijk maken; de zogenaamde 'omics of array-gebaseerde technieken. Deze technieken beloven een hogere gevoeligheid dan de conventionele toxicologische testen. Deze technieken zouden in staat moeten zijn om al een respons op blootstelling te detecteren bij lage dosissen nog voordat er een fenotypische verandering optreedt, wat de voorspellende waarde van deze technieken verhoogd [24-27]. Verder levert het vermogen van deze technieken om genoom-breed te meten de mogelijkheid op om de moleculaire mechanismen die ten 
grondslag liggen aan stof-gerelateerde reacties te bestuderen. Chips om genexpressie te bestuderen behoorden tot de eerste soort arrays die beschikbaar kwamen, maar die zijn nu aangevuld met onder andere miRNA en DNA-methylatie platvormen. Deze bieden de mogelijkheid om de epigenetische effecten van blootstelling aan stoffen te evalueren in toevoeging op de effecten op genexpressie niveau.

De hypothese in dit proefschrift is dat 'omics data afkomstig van in vitro modellen voor de humane lever ons in staat zullen stellen genen / sets van genen te definiëren die de variatie tussen individuen in de gevoeligheid voor blootstelling aan stoffen kunnen verklaren. Verder, zouden de data afkomstig van de epigenetische technieken ons in staat moeten stellen om te beoordelen of de epigenetische status van een individu de gevoeligheid van deze persoon betreffende de respons op blootstelling aan stoffen beïnvloedt.

\section{Evaluatie van interindividuele variatie met behulp van precisie-gesneden humane leverslices}

In Hoofdstuk $\mathbf{2}$ hebben we precisie-gesneden humane leverslices gebruikt om zo te bepalen of de interindividuele variatie in basale enzymactiviteit (EA) en genexpressie (GE) niveaus in de lever predisponeren voor de variatie in toxiciteits-responsen, gebaseerd op het bepalen van de dosis-respons relaties voor verschillende prototypische lever-toxische stoffen. Basale niveaus van CYP GE en EA werden gemeten in precisie-gesneden humane leverslices. Daarnaast werden slices blootgesteld aan een dosisreeks van paracetamol (APAP), aflatoxine B1 (AFB), benzo( $\alpha$ )pyreen (BaP) of 2-nitrofluoreen (NF). Interindividuele variatie in geïnduceerde genotoxiciteit (COMET-assay en CDKN1A/p21 GE) en cytotoxiciteit (afgifte van lactaatdehydrogenase), gecombineerd met NQO1-en GSTM1-GE-geïnduceerde reacties voor oxidatieve stress en GE-reacties van verschillende CYP werden geëvalueerd. De benchmark dosis (BMD)-benadering werd toegepast als een hulpmiddel om de blootstellingsresponsen te modelleren op individueel niveau.

Variatie in basale CYP-niveaus, zowel GE als EA, lijken de variatie in de respons op blootstelling aan stoffen op een individueel niveau te verklaren. De belangrijkste parameters die interindividuele variatie na blootstelling verklaren, kunnen worden gedefinieerd door netwerkanalyse. Met betrekking tot NF duidt deze analyse op de betrokkenheid van CYP1B1 in het metabolisme van deze verbinding wat een nieuwe bevinding vertegenwoordigt. Ook, GSTM1 waarvan bekend 
is dat het sterk varieert in expressie binnen de humane populatie, maar waarvan de variatie tot nu toe niet konden worden gekoppeld aan toxiciteit-responsen in patiënten vergiftigd door APAP, wordt in de netwerkanalyse geassocieerd met de interindividuele variabiliteit in APAP-metabolisme, afhankelijk van de individuele expressie van CYPenzymen.

Deze studie toont aan dat de inclusie van interindividuele variatie in netwerkmodellering een werkwijze creëert voor het bepalen van essentiële en nieuwe parameters geassocieerd met stof-gerelateerd metabolisme. Van alle verbindingen is de interindividuele variatie in de cytotoxiciteitsrespons gebaseerd op BMDs het grootste na APAPblootstelling (400-voudig). Deze hoge interindividuele variabiliteit komt overeen met bevindingen uit de literatuur betreffende de grote variabiliteit binnen de humane populatie ten aanzien van de gevoeligheid voor toxische reacties op APAP [28]. Bovendien, ligt deze variatie ook ver boven de 10-voudige marge die moet corrigeren voor interindividuele variatie binnen procedures voor humane risicoschatting. Deze informatie benadrukt de noodzaak van een grondigere evaluatie van interindividuele variatie in toxische reacties, met het oog op de nodige verbetering van kwantitatieve inschatting van de risico's voor een individu. Voor AFB, BaP en NF waren de verschillen in interindividuele variatie in de cytotoxiciteitsrespons afgeleid van BMDs veel lager (respectievelijk 6, 54, en 4-voudig).

\section{Interindividuele variatie in primaire humane hepatocyten}

Hoofdstuk 3 richt zich op de interindividuele variatie in genexpressie reacties en metabolietvorming in primaire humane hepatocyten $(\mathrm{PHH})$ blootgesteld aan APAP. APAP behoort tot de groep van receptvrije geneesmiddel en is een van de meest gebruikte analgetica/antipyretica wereldwijd [29, 30]. Hoewel grote interindividuele variatie in de gevoeligheid voor APAP-geïnduceerd leverfalen is beschreven, zijn de exacte onderliggende factoren die deze variabiliteit in gevoeligheid veroorzaken nog grotendeels onbekend [31, 32].

Om de variabiliteit in respons op APAP beter te begrijpen werden de interindividuele verschillen in genexpressie veranderingen en APAP-metabolietformatie in PHH van verschillende donoren in vitro blootgesteld aan een niet-toxische tot toxische APAP-dosisreeks geëvalueerd. De interindividuele variatie in gen expressie niveaus werd gecorreleerd aan de interindividuele variatie in APAP-metaboliet niveaus 
om te evalueren of verschillen in gen expressie niveaus de verschillen in APAP-metaboliet formatie niveaus zouden kunnen verklaren. Genenset over-representatie analyse werd toegepast om biologische interpretatie van de transcriptoom-gerelateerde resultaten mogelijk te maken.

De biologische processen waarin de genen met de grootste interindividuele variatie in expressie betrokken zijn, omvatten leverregeneratie, ontstekingsreacties, mitochondriële stress-respons, hepatocarcinogenese, celcyclus en medicatie-effectiviteit. Daarnaast zou de interindividuele variatie in de expressie van deze genen geassocieerd zijn met de variabiliteit in de metaboliet niveaus van hydroxyl-APAP, methoxy-APAP en C8H1305N-APAP-glucuronide. Deze genen/ metabolieten kunnen dus van belang zijn in de context van medicatiegeïnduceerde leverschade, vooral als ze kunnen worden bevestigd in een humane in vivo situatie zoals beschreven in Hoofdstuk 6. Bovendien lijkt de interindividuele variatie in genexpressie niveaus over het algemeen de variatie in metaboliet niveaus te overschrijden.

Zoals eerder vermeld, worden cellijnen tegenwoordig veel gebruikt als in vitro model voor de humane lever in toxicologisch onderzoek. Echter, deze cellijnen voorzien niet in de mogelijkheid tot de evaluatie van interindividuele variatie. In Hoofdstuk $\mathbf{4}$ evalueren we in welke mate de data afkomstig van verschillende cellijnen vergelijkbaar zijn met de data van $\mathrm{PHH}$, een in vitro model met interindividuele variatie. Hoewel eerdere studies voornamelijk gericht zijn op de vergelijking/toepasbaarheid van deze celtypes op basis van hun basale biotransformatie capaciteiten/CYP-induceerbaarheid [33-35], kozen wij voor een aanpak gebaseerd op een genoom-breed genexpressieprofiel, zowel op basaal niveau als op compound geïnduceerde genexpressie niveau. Daarvoor hebben we basale en AFB-en BaP-geïnduceerde genexpressie profielen in HepG2, HepaRG en PHH vergeleken.

Op basaal niveau verschillen levermodellen van elkaar wat betreft hun genoom-breed genexpressie niveau. PHH worden, zoals verwacht, gekenmerkt door grote interindividuele verschillen en zijn het meest vergelijkbaar met HepaRG. De genexpressie profielen geïnduceerd door blootstelling aan chemicaliën zijn meer vergelijkbaar tussen de cel modellen, vooral na BaP blootstelling. Processen betrokken bij het metabolisme van stoffen werden geïnduceerd in alle 3 de modellen, terwijl andere processen meer uitgesproken tot expressie komen in een bepaald cel model. Voorbeelden zijn transcriptomic modificaties van koolhydraat-gerelateerde genen (HepaRG) en receptor-gerelateerde genen $(\mathrm{PHH})$ na BaP blootstelling, en celcyclus-gerelateerde genen (HepG2) na AFB behandeling. Dus op basaal niveau, zijn PHH beter 
vergelijkbaar met HepaRG dan met HepG2, maar voor toepassing binnen de toxicogenomics lijken beide cellijnen even goed te presteren in vergelijking met $\mathrm{PHH}$. Echter, de interindividuele variatie in het $\mathrm{PHH}$ model zorgt voor een veel breder en meer heterogeen beeld in de respons op blootstelling. Omdat deze interindividuele variatie niet kan worden vastgesteld door de cellijn modellen, gaat hun toepassing binnen de beoordeling van toxiciteits-risico's gepaard met de kans op een onvolledige evaluatie met betrekking tot de human populatie. Nu we duidelijke interindividuele variatie in basale en stof-geïnduceerde genexpressie niveaus hebben vastgesteld, blijft de vraag wat de oorzaak is van deze interindividuele verschillen. De invloed van epigenetische factoren zoals gen-methylatie, zijn voorgesteld als bijkomende factoren die het verschil in geïnduceerde genexpressie niveaus bij de mens zouden kunnen verklaren. Daarom hebben we in Hoofdstuk $\mathbf{5}$ bekeken in hoeverre de basale methylatie-status van een gen de expressie van dat gen beïnvloed in respons op blootstelling aan verbinding. In dit verband wordt in het bijzondere de nadruk gelegd op de interindividuele variatie van de DNA-schade respons.

Van PHH van verschillende donoren blootgesteld aan AFB of BaP (zie Hoofdstuk 4) werd de respons op DNA-schade geëvalueerd door $\mu \mathrm{H} 2 \mathrm{Ax}$-kleuring. Deze resultaten werden gebruikt om de studiepopulatie in twee groepen te verdelen; een lage en een hoge responsgroep, gebaseerd op de BMD voor de DNA-schade respons. Genen uniek in hun genexpressie respons voor een van deze twee groepen werden beoordeeld op hun basale methylatie-status en basale genexpressie niveaus.

Hoewel duidelijke verschillen in de respons op DNA-schade tussen hoge en lage responsgroepen kunnen worden gedefinieerd, lijkt basale methylatie-status slechts minimaal bij te dragen aan een verklaring voor deze variatie. Ook lijkt interindividuele variatie in genexpressie op basaal niveaus niet te zijn gerelateerd aan de variatie tussen individuen in de basale gen methyleringsstatus, wat vervolgens weer niet wordt weerspiegeld in de variatie tussen individuen in genexpressie responsen. Dus, de algemene conclusie is dat de interindividuele variatie in de basale methyleringsstatus slechts beperkte aanvullende informatie biedt over de gevoeligheid voor blootstelling aan carcinogenen van groepen/individuen. Mogelijk zijn de mechanismen achter de regulatie van genexpressie door methylatie gecompliceerder dan momenteel erkend. De inclusie van andere (epi) genetische factoren, zoals miRNA's, zouden kunnen helpen ons vermogen tot interpretatie en begrip van interindividuele variatie te complementeren. 


\section{Interindividuele variatie in humane in vivo reacties}

Hoewel in vitro humane levermodellen dusver nuttig zijn gebleken om interindividuele variatie te evalueren is het ultieme model natuurlijk de in vivo humane lever. Echter, vanwege voor de hand liggende ethische bezwaren behoort het opzettelijk blootstellen aan toxische dosissen van verbinding niet tot de mogelijkheden met dit model. Verder is de toegankelijkheid van de lever beperkt vanwege de anatomische positie van dit orgaan. Echter, het is bewezen dat bloed als referentie of surrogaat weefsel gebruikt kan worden om leverfunctie te evalueren, ook na de blootstelling aan lichaamsvreemde stoffen [36-38].

In Hoofdstuk 6 hebben we de interindividuele verschillen in transcriptie reacties in bloedcellen van diverse vrijwilligers blootgesteld aan relatief lage, therapeutische dosissen APAP geëvalueerd. Een serie van standaard klinisch-chemische lever tests werd gebruikt om te controleren op mogelijke tekenen van leverschade. Een procedure vergelijkbaar met die in Hoofdstuk 2 werd gebruikt om genexpressie veranderingen na blootstelling aan APAP te evalueren. Ook interindividuele variatie in APAP-metaboliet vorming in bloed en urine werd bepaald. Voor zover bij ons bekend, is dit de eerste studie die de genoom-brede humane miRNA expressie veranderingen in bloedmonsters evalueert van gezonde vrijwilligers blootgesteld aan APAP.

Veel bekende, maar ook verschillende nieuwe APAPmetabolieten werden gedetecteerd, in het bijzonder met betrekking tot hepatotoxiciteit gerelateerde processen en het oxidatieve metabolisme van APAP. Ook de in Hoofdstuk $\mathbf{2}$ beschreven metabolieten die mogelijke interessant zijn in het perspectief van medicatie-geïnduceerde leverschade worden bevestigd in deze in vivo studie. Veranderingen in de transcriptie van genen wijzen op immuun-modulerende effecten en oxidatieve stress gerelateerde in een reactie op APAP blootstelling.

'Omics technieken blijken beter te presteren dan de klinischchemische tests en onthullen nieuwe processen in reactie op APAP bij de mens. Hoewel er geen definitieve conclusie over de mogelijke immuun-toxische effecten van APAP kan worden getrokken uit dit onderzoek, zijn er duidelijke aanwijzingen dat het immuunsysteem wordt geactiveerd, zelfs na inname van een lage dosis APAP. Ook, de betrokkenheid van oxidatieve stress gerelateerde genexpressie reacties vergelijkbaar met die gezien na blootstelling aan een hoge dosis APAP suggereert het optreden van mogelijke pre-toxische effecten van 
therapeutische APAP-doses. Mogelijk zijn deze effecten gerelateerd aan een dosis-afhankelijke verhoging van hepatotoxiciteit-gerelateerde metaboliet niveaus. Bovendien kan de interindividuele variatie miRNA expressiepatronen worden gerelateerd aan de interindividuele variatie in genexpressie reacties. Daarom moeten miRNA's worden beschouwd als een nuttige toevoeging op de kennis die de interindividuele variatie binnen de humane populatie kan verklaren.

\section{Conclusies}

Netwerk analyse (Hoofdstuk 2 ) is nuttig gebleken bij het bepalen van de basale CYP genexpressie en enzymactiviteit niveau als de belangrijkste parameters die interindividuele variatie na blootstelling beïnvloeden. De grote interindividuele variatie in metabole genen, vooral CYP, word bevestigd in Hoofdstuk 5.

$\mathrm{PHH}$ zijn op basaal genexpressie niveau, verschillend van HepaRG en HepG2, twee-carcinoom afgeleide cel modellen die momenteel worden gebruikt in toxiciteit testen (Hoofdstuk 4). PHH, HepaRG en HepG2 worden meer vergelijkbaar met betrekking tot hun genexpressie profielen na blootstelling aan verbinding. Toch geeft het $\mathrm{PHH}$-model een veel breder en meer heterogeen beeld van de reacties op blootstelling aan verbinding door de inclusie van de interindividuele variatie tussen donoren.

Interindividuele variatie in genexpressie reacties konden worden gedetecteerd in alle niet carcinoma gebaseerde in vitro en in vivo levermodellen toegepast in dit proefschrift (Hoofdstuk 2,3 en 6). Interindividuele variatie in sommige parameters overschrijdt de 10voudige marge die wordt verondersteld de interindividuele variatie binnen de humane populatie te vertegenwoordigen in toxiciteitstesten. Deze factoren omvatten BMDs gebaseerd op genexpressie reacties en cytotoxiciteit reacties, maar ook niveaus van metabolietvorming. Daarnaast lijkt de inclusie van data over interindividuele variatie in miRNA expressie niveaus behulpzaam zijn bij het verklaren van de grote variatie tussen individuen in reactie op APAP blootstelling (Hoofdstuk 6).

De combinatie van meerdere 'omics technieken (bijv. genomics en proteomics) is zeer nuttig gebleken bij het bepalen van metabolieten die kunnen worden gebruikt om interindividuele variatie in de respons op APAP-blootstelling (Hoofdstuk 3 en $\mathbf{6}$ ) te monitoren. De toevoeging van epigenetische gegevens in een poging om interindividuele variatie 
verklaren was succesvoller betreffende de toevoeging van informatie over miRNA expressie dan met de toevoeging van methylatiegerelateerde data (Hoofdstuk 5 en 6).

Concluderend, bevestigt het onderzoek in dit proefschrift de aanwezigheid van aanzienlijke interindividuele variatie in de reacties op blootstelling aan toxische stoffen binnen de humane populatie. Basale CYP enzymactiviteit en genexpressie niveaus, als ook chemisch geïnduceerde genotoxiciteit, cytotoxiciteit en genexpressie niveaus vertonen allen duidelijke variatie tussen levercellen van individuele donors. Naar onze mening zou de implementatie van kennis over humane interindividuele variatie moeten leiden tot de ontwikkeling van minder willekeurige procedures voor de schatting van humane risico's en zou een beter begrip van de moleculaire mechanismen die ten grondslag liggen aan de reacties op verbindingen verkregen moeten kunnen worden.

\section{Aanbeveling voor toekomstige richtingen}

Tijdens de laatste jaren is het besef voor het belang van de evaluatie van interindividuele variatie toegenomen, samen met een oplopende vraag naar de vervanging van proefdiermodellen. Echter, om volledig te kunnen begrijpen hoe interindividuele variatie toxiciteitsreacties beïnvloed zullen grote hoeveelheden data van een grote groep van individuen verzameld moeten worden om zo een dataset te kunnen creëren die een betrouwbare vertegenwoordiging van de heterogeniteit binnen de humane populatie weerspiegeld. Hiervoor is een relevant humaan cel model dat gemakkelijk toegankelijk en op grote schaal toepasbaar is noodzakelijk. Betreffende levermodellen is dit (nog) niet beschikbaar. De bestaande cellijnen die wel talrijk beschikbaar en goedkoop zijn, houden geen rekening met interindividuele variatie. $\mathrm{PHH}$ en lever slices zijn niet beschikbaar in grote hoeveelheden en komen met hoge technische eisen.

Onlangs werden verschillende nieuwe in vitro modellen ontwikkeld. Een van deze modellen is gebaseerd op hepatocytachtige cellen die kunnen worden verkregen uit de differentiatie van humane embryonale stamcellen [39]. Een vergelijkbaar model is het is het humane geïnduceerde pluripotente stamcel-model [40]. Dit model heeft als voordeel dat de genetische achtergrond van de cellen afkomstig van de stamcellen dicht bij de genetische achtergrond van normale hepatocyten ligt. Verder zijn de technieken om cellen te kweken verbeterd, waardoor voor het kweken van cellen in 
3D- of microweefsel formatie tot de mogelijkheden behoort. Deze nieuwe kweektechnieken zouden de natuurlijke omgeving van de cel in de lever na moeten bootsen en op die manier onder andere een verbetering van de metabole capaciteit van de cellen teweeg moeten brengen in vergelijking met de normale 2D-kweken [40]. Bovendien zijn zogenaamde co-kweken ontwikkeld die zowel primaire parenchym en niet-parenchymale levercellen bevatten. Ook deze cel modellen vertegenwoordigen een in vivo-achtige weergave van de lever met verbeterde functionele mogelijkheden [41]. In alle hierboven genoemde nieuwe in vitro modellen voor de lever behoort de evaluatie van interindividuele variatie tot de mogelijkheden waardoor een veelbelovend toekomstbeeld wordt geschapen

Echter de grootste uitdaging ligt waarschijnlijk in de nodige aanpassingen van de wetten en regelgevingen. Dit geldt in het algemeen voor het testen van toxiciteit, maar is in het bijzonder van toepassing op de wetten en regelgevingen die gepaard gaan met de humane risicobeoordeling. Elk nieuw model zal moeten concurreren met de test die overtijd de gouden standaard hebben gevormd, zoals de proefdiermodellen. Dit zal zonder twijfel een zeer langdurig proces blijken te zijn. 


\section{References}

1. Omiecinski, C.J., et al., Xenobiotic metabolism, disposition, and regulation by receptors: from biochemical phenomenon to predictors of major toxicities. Toxicol Sci, 2011. 120 Suppl 1: p. S49-75.

2. Urquhart, B.L., R.G. Tirona, and R.B. Kim, Nuclear receptors and the regulation of drug-metabolizing enzymes and drug transporters: implications for interindividual variability in response to drugs. J Clin Pharmacol, 2007. 47(5): p. 566-78.

3. Johansson, I. and M. Ingelman-Sundberg, Genetic Polymorphism and Toxicology-With Emphasis on Cytochrome P450. Toxicological Sciences, 2011. 120(1): p. 1-13.

4. Daly, A.K., Using genome-wide association studies to identify genes important in serious adverse drug reactions. Annu Rev Pharmacol Toxicol, 2012. 52: p. 21-35.

5. Guengerich, F.P., Cytochrome P450s and other enzymes in drug metabolism and toxicity. AAPS J, 2006. 8(1): p. E101-11.

6. Guengerich, F.P., Cytochrome p450 and chemical toxicology. Chem Res Toxicol, 2008. 21(1): p. 70-83.

7. Hussaini, S.H. and E.A. Farrington, Idiosyncratic drug-induced liver injury: an overview. Expert Opin Drug Saf, 2007. 6(6): p. 673-84.

8. Bogen, K.T. and R.C. Spear, Integrating uncertainty and interindividual variability in environmental risk assessment. Risk analysis : an official publication of the Society for Risk Analysis, 1987. 7(4): p. 427-36.

9. Lehman, A.J. and O.G. Fitzhugh, 100-fold margin of safety. Assoc.Food Drug Off. U.S.Q. Bull., 1954. 18: p. 33-35.

10. European-Commission, Opinion of the Scientific Committee on Toxicity, Ecotoxicity and the Environment on The BUAV-European Coalition to End Animal Experiments Report: The Way Forward - Action to End Animal Toxicity Testing, 2004.

11. The-Staff-of-ACSH, Of Mice and Mandates: Animal Experiments, Human Cancer Risk, and Regulatory Policies, 1997.

12. Dorne, J.L.C.M. and A.G. Renwick, The Refinement of Uncertainty/Safety Factors in Risk Assessment by the Incorporation of Data on Toxicokinetic Variability in Humans. Toxicological Sciences, 2005. 86(1): p. 20-26.

13. KEMI-Karolinska-Institute-Sweden. HUMAN HEALTH RISK ASSESSMENT Proposals for the use of assessment (uncertainty) factors; Application to risk assessment for plant protection products, industrial chemicals and biocidal products within the European Union. 2003; Available from: http://www.kemi. se/Documents/Publikationer/Trycksaker/Rapporter/Rapport1_03.pdf.

14. Levy, N., The use of animal as models: ethical considerations. International journal of stroke : official journal of the International Stroke Society, 2012. 7(5): p. 440-2.

15. Fasinu, P., P.J. Bouic, and B. Rosenkranz, Liver-based in vitro technologies for drug biotransformation studies - a review. Curr Drug Metab, 2012. 13(2): p. 215-24.

16. Knowles, B.B., C.C. Howe, and D.P. Aden, Human hepatocellular carcinoma cell lines secrete the major plasma proteins and hepatitis B surface antigen. Science, 1980. 209(4455): p. 497-9.

17. Morris, K.M., et al., Complement biosynthesis by the human hepatoma-derived cell line HepG2. J Clin Invest, 1982. 70(4): p. 906-13.

18. Aninat, C., et al., Expression of cytochromes P450, conjugating enzymes and 
nuclear receptors in human hepatoma HepaRG cells. Drug Metab Dispos, 2006. 34(1): p. 75-83.

19. Cerec, V., et al., Transdifferentiation of hepatocyte-like cells from the human hepatoma HepaRG cell line through bipotent progenitor. Hepatology, 2007. 45(4): p. 957-67.

20. Guillouzo, A., et al., The human hepatoma HepaRG cells: a highly differentiated model for studies of liver metabolism and toxicity of xenobiotics. Chem Biol Interact, 2007. 168(1): p. 66-73.

21. de Graaf, I.A., et al., Preparation and incubation of precision-cut liver and intestinal slices for application in drug metabolism and toxicity studies. Nature protocols, 2010. 5(9): p. 1540-51.

22. Kmiec, Z., Cooperation of liver cells in health and disease. Adv Anat Embryol Cell Biol, 2001. 161: p. III-XIII, 1-151.

23. Treyer, A. and A. Musch, Hepatocyte polarity. Compr Physiol, 2013. 3(1): p. 243-87.

24. Beger, R.D., J. Sun, and L.K. Schnackenberg, Metabolomics approaches for discovering biomarkers of drug-induced hepatotoxicity and nephrotoxicity. Toxicol Appl Pharmacol, 2010. 243(2): p. 154-66.

25. Harrill, A.H. and I. Rusyn, Systems biology and functional genomics approaches for the identification of cellular responses to drug toxicity. Expert Opin Drug Metab Toxicol, 2008. 4(11): p. 1379-89.

26. Vinayavekhin, N., E.A. Homan, and A. Saghatelian, Exploring disease through metabolomics. ACS Chem Biol, 2010. 5(1): p. 91-103.

27. Vlaanderen, J., et al., Application of OMICS technologies in occupational and environmental health research; current status and projections. Occup Environ Med, 2010. 67(2): p. 136-43.

28. Spielberg, S.P., Acetaminophen toxicity in human lymphocytes in vitro. The Journal of pharmacology and experimental therapeutics, 1980. 213(2):

p. 395-8.

29. Lee, W.M., Acetaminophen toxicity: changing perceptions on a social/medical issue. Hepatology, 2007. 46(4): p. 966-70.

30. Kuehn, B.M., FDA focuses on drugs and liver damage: labeling and other changes for acetaminophen. JAMA, 2009. 302(4): p. 369-71.

31. Prescott, L.F., Paracetamol overdosage. Pharmacological considerations and clinical management. Drugs, 1983. 25(3): p. 290-314.

32. Rannug, U., et al., An evaluation of the genetic toxicity of paracetamol. Mutation Research/Fundamental and Molecular Mechanisms of Mutagenesis, 1995. 327(1-2): p. 179-200.

33. Hart, S.N., et al., A comparison of whole genome gene expression profiles of HepaRG cells and HepG2 cells to primary human hepatocytes and human liver tissues. Drug Metab Dispos, 2010. 38(6): p. 988-94.

34. Jennen, D.G., et al., Comparison of HepG2 and HepaRG by whole-genome gene expression analysis for the purpose of chemical hazard identification. Toxicol Sci, 2010. 115(1): p. 66-79.

35. Gerets, H.H., et al., Characterization of primary human hepatocytes, HepG2 cells, and HepaRG cells at the mRNA level and CYP activity in response to inducers and their predictivity for the detection of human hepatotoxins. Cell Biol Toxicol, 2012. 28(2): p. 69-87.

36. Liew, C.-C., et al., The peripheral blood transcriptome dynamically reflects system wide biology: a potential diagnostic tool. Journal of Laboratory and Clinical Medicine, 2006. 147(3): p. 126-132.

37. Mohr, S. and C.-C. Liew, The peripheral-blood transcriptome: new insights into 
disease and risk assessment. Trends in Molecular Medicine, 2007. 13(10): p. 422-432.

38. Rockett, J.C., et al., Surrogate tissue analysis: monitoring toxicant exposure and health status of inaccessible tissues through the analysis of accessible tissues and cells. Toxicology and applied pharmacology, 2004. 194(2): p. 189-99.

39. Yildirimman, R., et al., Human embryonic stem cell derived hepatocyte-like cells as a tool for in vitro hazard assessment of chemical carcinogenicity. Toxicol Sci, 2011. 124(2): p. 278-90.

40. Mathur, A., et al., Human induced pluripotent stem cell-based microphysiological tissue models of myocardium and liver for drug development. Stem cell research \& therapy, 2013. 4 Suppl 1: p. S14.

41. Kostadinova, R., et al., A long-term three dimensional liver co-culture system for improved prediction of clinically relevant drug-induced hepatotoxicity. Toxicol Appl Pharmacol, 2013. 268(1): p. 1-16. 


\section{Addendum I"}

\section{Valorization}

The valorization of scientific knowledge comprises a process that should ensure the creation of value from knowledge by enhancing the availability and/or suitability of knowledge for social and/or economic purposes as such translating knowledge into for instance products, services or new activities. Here, the options for valorization of the work described in this thesis will be discussed, by highlighting:

- $\quad$ the relevance of the work,

- $\quad$ the target groups to whom the work applies,

- $\quad$ activities/product/services that can be derived from the work,

- options for innovation of the results

- $\quad$ the implementation of the valorization process.

\section{Relevance}

The work in this thesis was largely preformed under the carcinoGENOMICS project (FP6 project from the European Union) [1] and the ASAT program ('Assuring Safety without Animal Testing' an initiative from the Dutch ministry of Health, Welfare and Sports) [2]. Both projects are concerned with the 3 R's in animal testing [3]. Thus, these projects aim at contributing towards the Replacement, Reduction and Refinement of animal testing.

Societal resistance against the use of animal models steadily increases due to the ethical and moral dilemmas paired with the use of these models [4]. Nevertheless, animal models have been adopted as the 'golden standard' in many applications, including toxicity testing of chemical compounds and medical drugs. For the preservation of the current levels of human health and environmental protection many regulatory bodies emphasize the need for toxicity testing in animal models [5]. As a result, the use of animal models has become deeply manifested into the rules and regulations with respect to toxicity testing, as such becoming an obligatory hurdle to take for ensuring human safety [6]. Animal models are labor-intensive and often need to be maintained over long timespans, which comes along with a heavy financial burden [4]. In addition, over the recent years, the reliability and applicability of animal model-derived data for the human situation has been questioned [4]. Animal data used to predict human safety, 
resulted in a correct prediction in about $50-70 \%$, which in some cases thus is barely better than flipping a coin [7]. As a result of incorrect prediction of human safety, there is the risk of admitting dangerous chemicals (including but not limited to industrial chemicals, pesticides and drugs) to the market. On the other hand, incorrect prediction might also lead to the rejection of truly innocent compounds from the market, which could have had beneficial applications for humans, for instance as a drug. One of the factors that might be influencing the limited applicability of animal model-derived information towards the human situation is the well-known large interindividual variation within the human population in response to chemical exposure $[8,9]$.

All abovementioned arguments demonstrate the need for models that preferably omit the use of animals, while having an increased applicability for the human situation. Against this background, in this thesis, the performance of several human in vitro models was assessed and their usefulness for the evaluation of toxicity responses was proven. Therefore, the work described in this thesis, is of relevance in the efforts being taken to decrease the burden on animal testing.

Although interindividual variation in toxicity responses within the human population is a well-known fact, the amount of variation with respect to toxicity related parameters is largely unknown. In procedures for human risk-estimation, interindividual variation is dealt with in a very arbitrary way by applying a simple 10-fold factor to data in order to correct for human interindividual variation. An overestimation of interindividual variation through this factor, i.e. the actual interindividual variation is lower than 10 -fold, could for instance lead to unnecessarily strict restrictions on the allowable dose-range of a pharmaceutical intended for human use. On the other hand, in case the 10-fold margin is an underestimation of the actual interindividual variation, sensitive individuals within the population could be exposed to a dose of a compound/xenobiotic that imposes a threat to their health/safety. The cell models used within this thesis all allow for the evaluation of interindividual variation and therefore can contribute to the knowledge about the amount of interindividual variation in toxicity responses. By gaining this knowledge, the development of less arbitrary and more precise methods to integrate interindividual variation in human risk-estimation procedures should become possible. This in turn should ensure an improvement of the safety of the human population concerning toxicity related risks. 


\section{Target groups}

The pharmacological industry, the cosmetic industry and the chemical industry can be defined as several prime user groups for whom the outcome of this thesis might be of interest. The opportunities for the valorization of the knowledge obtained from this thesis within each of the defined target groups will be discussed below.

- $\quad$ Pharmacological industry

Within the pharmacological industry 'omics techniques, as the ones used within this thesis, are already regularly applied to define compounds of interest early on in the developmental process. All of these possibly interesting compounds need to be tested for potential therapeutic and adverse effects. Many compounds will eventually fail due to toxicity problems in later developmental phases, leading to huge amounts of resources being spilled. In addition, withdrawals of drugs from the market due to adverse effects that were not detected during the testing phase, have a disastrous effect on the reputation and economic status of a company. This also indicates that the current, mainly animal-based models used to test for adverse effects of a compound still need to be improved. Which is where the knowledge obtained through the work described in thesis, may provide options for improvement. In addition, understanding of the causes of interindividual variation within the human population could help pharmacological industry by enabling the identification of susceptible groups within the human population. Also, whit a better understanding of the causes of interindividual variation, one should be able to unravel the molecular mechanisms behind this variation. For the pharmaceutical industry this enables possibilities for a more targeted approach in drug development, as such decreasing time and resources spend on the development of unsuccessful compounds.

\section{- $\quad$ Cosmetic industry}

Within the cosmetic industry, there is an urgency to develop and implement alternative non-animal based in vitro safety testing tools. This is a consequence of the implementation of the EU 7th amendment, which has led to the gradual ban of animalbased testing for cosmetics [10]. Although toxicogenomicsbased research is being applied to some extent within the cosmetic industry, there is a general unawareness of the 
potential (and limitations) of toxicogenomics-related research within this industry. The knowledge obtained within this thesis, may very well be transferable to alternatives to animal models that are being developed for use within the cosmetics industry and as such may provide further insight in the capacities of these new models. The same issues concerning human interindividual variation as described for the pharmaceutical industry also apply to the cosmetic industry, although they might be of less primary concern within this target group.

- $\quad$ Chemical industry

Chemical safety assessment is an integral part of the chemical industry. Many rules and regulations are in place to ensure human safety. One example is the REACH (Registration, Evaluation, Authorization and Restriction of Chemicals) initiative by the European Union (EU), which now holds chemical industry responsible for the assessment and management of the risks caused by chemicals, and for the communication of those risks to users [11]. This should ensure the protection of human health and the environment from the risks that can be posed by chemicals. Clearly, knowledge on interindividual variation within the human population, as can be derived from the models used within this thesis, could provide additional insight by the definition of susceptible individuals and/or mechanistic information on adverse responses to chemicals. In addition the REACH program promotes the use and development of alternative, non-animal based test methods. As mentioned before, the cell models used in this thesis are all human-based models that could possibly provide such an alternative to animal models.

\section{Activities/Products/Services}

Although the knowledge obtained from the research within this thesis is still preliminary, there is a strong social and political drive, both on a national and international level, behind further exploration of alternative methods for animal-testing. This includes opportunities for the improvement of currently available non-animal based testing methods, like the development of better in vitro test models that give a more accurate representation of the human situation than the currently available models. 
This thesis provides further insight in several human-based test models that allow for the evaluation of human interindividual variation. As such, this thesis services the ongoing development of these models in which a lot of knowledge is still lacking. Several alternative analytical tools, like the application of Bayesian network analysis to a combination of toxicity related parameters and the evaluation of baseline interindividual variation in epigenetic parameters, were applied in this thesis. These new approaches should service the further enrichment of analytical tools that will become necessary in future research. In addition, the research performed in this thesis should service the development of better statistical approaches that make optimal use of data.

Due to the high political interest in the development of other, better models for application to the human situation, funding for further research into this topic should be expected to become available both from governmental (national and international) agency's as from company/industry related sources.

\section{Innovation}

In this thesis several human in vitro models were compared for their response to exposure to (sub-)toxic doses of one or more compounds. Interindividual variation in these responses, not in the least caused by the genetic variation between individuals within the human population, were clearly present. In the current methods for human risk estimation, a simple extrapolation factor of 10 -fold is used to correct for interindividual variation. This 10 -fold factor is in general based on ancient data derived from primal testing methods. The data derived from the research with this thesis, based on newer more sophisticated measuring methods, show that this 10 -fold factor is far away from the actual variation seen between individuals.

In our opinion, any newly developed models for testing should thus allow for assessment of interindividual variation. As such more knowledge of the actual interindividual variation within the human population will gradually become available. This knowledge can then for instance be used to define groups or even individuals that are more or less susceptible to the side-effects of drugs. Many drugs show unwanted, severe side effects in only a small part of the human population, while they are having beneficial, therapeutic effects without side effects in a much larger part of the human population. If, whit increased knowledge on interindividual variation, individuals/groups 
whit increased susceptibility can be predefined; these drugs can be applied in a safer manner by omitting dosing to susceptible individuals/ groups. Thereby, a better understanding of the mechanisms behind the causes of interindividual variation that thus influence susceptibility should allow for a better definition of safe dose ranges of drugs.

In the recent past, personalized medicine aimed at the customization of healthcare tailored to the individual patient became a hot topic. Any information on interindividual variation and the causes behind this variation is of great value and should as such lead to further progress within this field.

\section{Implementation}

As described above in the paragraph on 'Activities/Products/ Services' some of the knowledge obtained from the work described in this thesis has already been implemented. New projects have been created based on the experiences obtained through the performed work. However, the long-term goal being the development of alternative testing methods and a better definition of interindividual variation as such and the causative mechanisms behind this variation will take some more investment both financially and time-wise.

Governmental agencies are facing political pressure to act proactive concerning the acceptance and implementation of alternatives to classical, animal based testing. Nevertheless, the uncertainty with novel assays makes regulators to be skeptical. While the general public and the market request more and better safety reporting, regulators on the other hand are becoming more careful in allowing a new test to enter this market, as they require fully validated assays that are reliable, robust and provide addition insight in comparison to the already available assays.

Thus probably, the largest issue to overcome will be the adaptation of the rules and regulations that apply to toxicity testing in general and human risk-estimation. Any new model will have to compete against the test that have become the golden standard overtime, like the animal models. This, without a doubt, will prove to be a very lengthy process. It has been estimated that the actual development of assays for predictive toxicology that are found suitable for regulatory purposes may take another 5-10 years. 


\section{References}

1. Vinken, M., et al., The carcinoGENOMICS project: critical selection of model compounds for the development of omics-based in vitro carcinogenicity screening assays. Mutat Res, 2008. 659(3): p. 202-10.

2. Chapin, R.E., et al., Assuring safety without animal testing: The case for the human testis in vitro. Reproductive Toxicology, 2013. 39(0): p. 63-68.

3. Russell, W.M.S. and R.L. Burch, The principles of humane experimental tech nique1959: Methuen.

4. Levy, N., The use of animal as models: ethical considerations. International journal of stroke : official journal of the International Stroke Society, 2012. 7(5): p. 440-2.

5. European-Commission, Opinion of the Scientific Committee on Toxicity, Eco toxicity and the Environment on The BUAV-European Coalition to End Animal Experiments Report: The Way Forward - Action to End Animal Toxicity Testing, I 2004.

6. The-Staff-of-ACSH, Of Mice and Mandates: Animal Experiments, Human Cancer Risk, and Regulatory Policies, 1997.

7. Olson, H., et al., Concordance of the Toxicity of Pharmaceuticals in Humans and in Animals. Regulatory Toxicology and Pharmacology, 2000. 32(1):

p. 56-67.

8. Omiecinski, C.J., et al., Xenobiotic metabolism, disposition, and regulation by receptors: from biochemical phenomenon to predictors of major toxicities. Toxicol Sci, 2011. 120 Suppl 1: p. S49-75.

9. Urquhart, B.L., R.G. Tirona, and R.B. Kim, Nuclear receptors and the regulation of drug-metabolizing enzymes and drug transporters: implications for interindi vidual variability in response to drugs. J Clin Pharmacol, 2007. 47(5): p. 566-78.

10. Adler, S., et al., Alternative (non-animal) methods for cosmetics testing: current status and future prospects-2010. Arch Toxicol, 2011. 85(5): p. 367-485.

11. Oltmanns, J., et al., The impact of REACH on classification for human health hazards. Regul Toxicol Pharmacol, 2014. 


\section{Dankwoord}

Aangekomen bij het dankwoord, misschien wel het meest populaire onderdeel van een proefschrift. Maar wel ergens helemaal achteraan op de laatste pagina's. Ik schrijf toe naar de laatste regels, komma's en punten. Dadelijk is het klaar, een hele opluchting en dat geldt heus niet voor mij alleen. Want er waren veel meer personen betrokken bij het ontstaan van dit proefschrift. Zonder hun bijdrages, groot of klein en in wat voor een vorm dan ook was het niet mogelijk geweest om tot dit eindresultaat te komen.

Normaal gezien zou er nu een opsomming volgen van namen en bijdrages met de bijbehorende dankbetuigingen, maar daar begin ik niet aan. Daarmee hoop ik te voorkomen mij op glad ijs te begeven, want wat als je iemand vergeet, de spelling van een naam verprutst, etc. Ik mag hopen dat iedereen die in de afgelopen jaren zijn of haar bijdrage heeft geleverd weet dat ik ze dankbaar ben.

Mochten er toch personen zijn die na het lezen van dit dankwoord nog de behoefte hebben aan een persoonlijk woordje van dank, kom dan gerust even bij mij langs en dan zal ik mijn uiterste best doen in die behoefte te voorzien.

Rest mij om nog eenmaal het woord te richten tot eenieder die op wat voor een manier dan ook heeft bijgedragen aan dit proefschrift; Hartelijk dank!

Grtz!

Marlon.
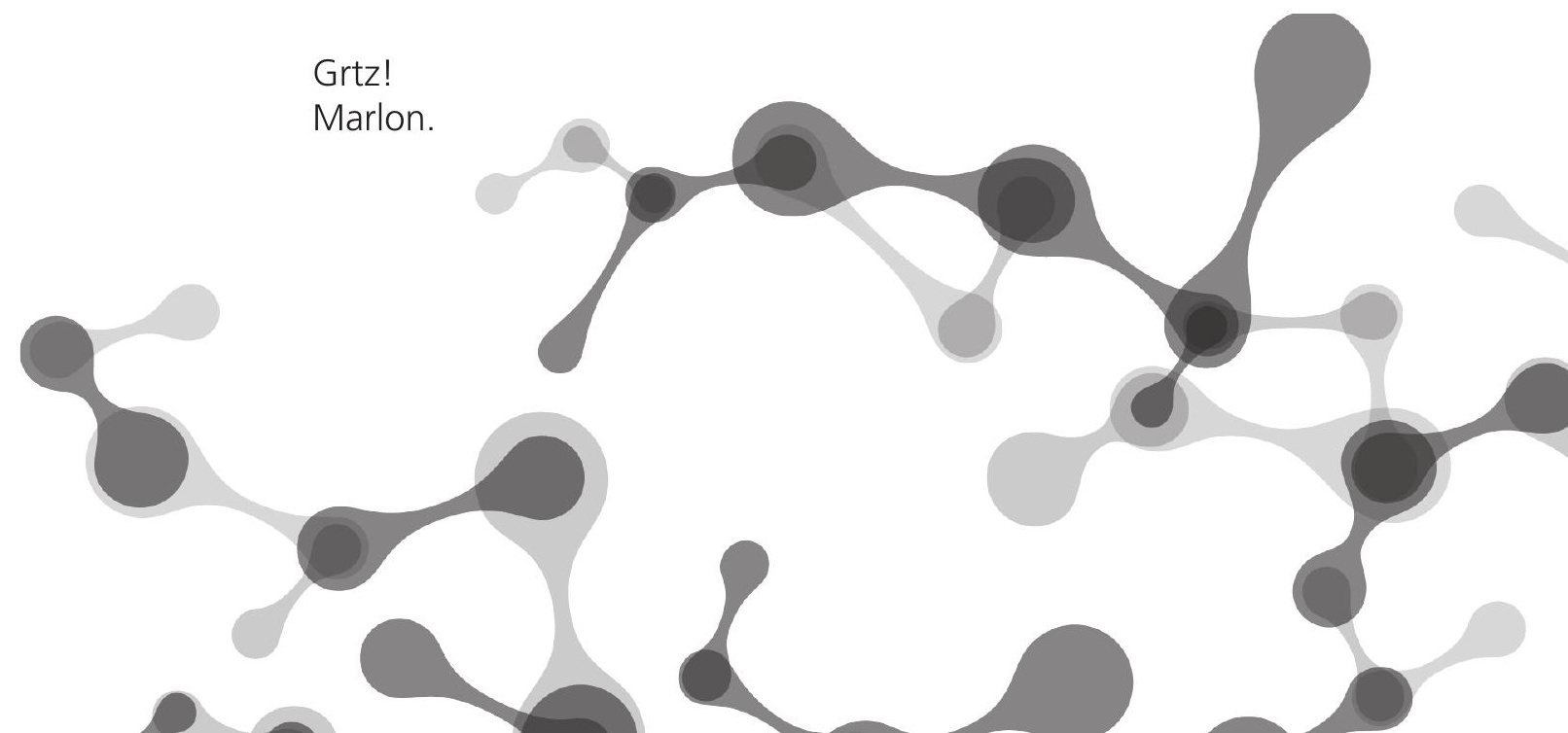


\section{Curriculum Vitae}

Marlon Jacoba Anne Jetten was born in Roermond (the Netherlands) on June the 24th in 1983. After finishing secondary school at the Stedelijk Lyceum in Roermond in 2000, she started her bachelor study 'Applied Sciences' at the Fontys Hogeschool Eindhoven. During this bachelor she did two internships at Maastricht University; one at the department of Clinical Genetics on 'The percentage of point mutations in mitochondrial DNA from single muscle cells from Leigh's disease patients' and another one at the department of Population Genetics on 'Optimization of the RNA-isolation procedure from biopsy material for use in microarray studies'.

In 2005 she started a two-year research master in 'Clinical Molecular Sciences' at the transnational University Limburg (tUL). During this master she did another two internships at the University of Maastricht, the first one at the Department of Anatomy \& Embryology focusing on 'The effects of arginine depletion due to arginase-1 over-expression in suckling F/A2 mice'. And a final internship at the Department of Psychiatry and Neuropsychology where she was involved in a project on 'The effects of prenatal stress on anxiety and depression related behavior and cognition in normal C57Bl6 mice'. Hereafter, in September 2007, she graduated cum laude and officially obtained her Master title.

In September 2007 she joined the Department of Toxicogenomics (TGX) at Maastricht University as a PhD-student. Here she was involved in several national and international projects; 'The potential of genomics technologies' and 'Transcriptomics analysis of human hepatocytes exposed to different concentrations of acetaminophen' both projects of the ASAT (Assuring Safety without Animal Testing) Innovation Program by the Dutch Ministry of Health, Welfare and Sport, and also in 'A view on human inter-individual variation and susceptibility' as a part of the CarcinoGENOMICS project by the European Union.

In June 2012 she continued her career at TGX by participating in another project from the European Union, 'The Data Infrastructure for Chemical Safety' (diXa). As from October 2014 she will be working on a postdoc project at TGX in collaboration with Exxon Mobile focusing at chemical risk assessment using several in vitro human skin models. 


\section{List of Publications}

Interindividual variation in response to xenobiotic exposure established in precision-cut human liver slices

Marlon J.A. Jetten, Sandra M. Claessen, Cornelis H.C. Dejong, Agustin Lahoz, José V. Castell, Joost H.M. van Delft and Jos C.S. Kleinjans. Published: Toxicology: Volume 223, September 2014, Pages 61-69

Interindividual variation in gene expression responses and metabolite formation in Acetaminophen-exposed primary human hepatocytes

Marlon J.A. Jetten, Ainhoa Ruiz-Aracama, Maarten L.J. Coonen, Sandra M. Claessen, Marcel H.M. van Herwijnen, Arjen Lommen, Joost H.M. van Delft, Ad A.C.M. Peijnenburg and Jos C.S. Kleinjans.

[Submitted]

Baseline and genotoxic compound induced gene expression profiles in HepG2 and HepaRG compared to primary human hepatocytes

Marlon J.A. Jetten, Jos C.S. Kleinjans, Sandra M. Claessen, Christophe Chesné and Joost H.M. van Delft.

Published: Toxicology In Vitro: Volume 27, Issue 7, October 2013, Pages 2031-2040

Does the epigenome predispose for susceptibility to DNA damage?

Marlon J.A. Jetten, Sandra M. Claessen, Hans Gmuender, Joost H.M. van Delft, Timo Wittenberger and Jos C.S. Kleinjans.

[In preparation]

'Omics analysis of low dose acetaminophen intake demonstrates novel response pathways in humans

Marlon J.A. Jetten, Stan Gaj, Ainhoa Ruiz-Aracama, Theo M.C.M. de Kok, Joost H.M. van Delft, Arjen Lommen, Eugene P. van Someren, Danyel G.J. Jennen, Sandra M. Claessen, Ad A.C.M. Peijnenburg, Rob H. Stierum and Jos C.S. Kleinjans.

Published: Toxicology and Applied Pharmacology: Volume 259, Issue 3, 15 March 2012, Pages 320-328 


\section{Other publications:}

Evaluation of database-derived pathway development for enabling biomarker discovery for hepatotoxicity.

Dennie G.A.J. Hebels, Marlon J.A. Jetten, Hugo J.W.L. Aerts, Ralph Herwig, Daniël H.J. Theunissen, Stan Gaj, Joost H.M. van Delft and Jos C.S. Kleinjans.

Biomark Med. 2014 Feb;8(2):185-200. doi: 10.2217/bmm.13.154 